\title{
New Derivatives and Iron \\ Complexes of the Siamese-Twin \\ Porphyrin
}

Dissertation

zur Erlangung des mathematisch-naturwisschenschaftlichen Doktorgrades

"Doctor rerum naturalium"

der Georg-August-Universität Göttingen

im Promotionsprogramm Chemie

der Georg-August University School of Science (GAUSS)

vorgelegt von

Oliver Mitevski

aus Hannover

Göttingen, 2016 
Betreuungsausschuss:

Prof. Dr. Franc Meyer, Institut für Anorganische Chemie, Georg-August-Universität Göttingen

Prof. Dr. Sven Schneider, Institut für Anorganische Chemie, Georg-August-Universität Göttingen

$\underline{\text { Mitglieder der Prüfungskommission }}$

Referent: Prof. Dr. Franc Meyer, Institut für Anorganische Chemie, Georg-AugustUniversität Göttingen

Korreferent: Prof. Dr. Sven Schneider, Institut für Anorganische Chemie, Georg-AugustUniversität Göttingen

Weitere Mitglieder der Prüfungskommission:

Prof. Dr. Dietmar Stalke, Institut für Anorganische Chemie, Georg-August-Universität Göttingen

Dr. Inke Siewert, Institut für Anorganische Chemie, Georg-August-Universität Göttingen

Dr. Alexander Breder, Institut für Organische und Biomolekulare Chemie, GeorgAugust-Universität Göttingen

Prof. Dr. Claudia Höbartner, Institut für Organische und Biomolekulare Chemie, GeorgAugust-Universität Göttingen

Tag der mündlichen Prüfung: 18.10.2016 
Ich versichere hiermit, dass ich die vorliegende Doktorarbeit selbstständig verfasst und keine anderen als die angegebenen Quellen und Hilfsmittel benutzt habe.

Ort, Datum

Unterschrift

Teile dieser Arbeit wurden bis jetzt in folgenden Artikeln veröffentlicht. Eine Genehmigung zur Verwendung von Textauszügen und Bildern liegt vor.

"Siamese-Twin Porphyrins: Variation of Two meso-Aryl Groups", O. Mitevski, S. Dechert, C. Brückner, F. Meyer Eur. J. Inorg. Chem. 2016, 4814-4819. DOI: 10.1002/ejic.201600714 

"The great tragedy of Science - the slaying of a beautiful hypothesis by an ugly fact."

Thomas H. Huxley, Biologist, 1825-1895 



\section{Contents}

1 General Introduction $\quad 1$

1.1 Porphyrins . . . . . . . . . . . . . . . . . . . . . . . 1

1.2 Expanded Porphyrins . . . . . . . . . . . . . . . . . 2

1.2.1 Structure and Nomenclature . . . . . . . . . . . . . . . . . 3

1.2.2 Metal Complexes . . . . . . . . . . . . . . . . . . . . 3

1.3 The Siamese-Twin Porphyrin $(\mathrm{STP}) \ldots \ldots \ldots \ldots$. . . . . . . . . 4

1.3 .1 Structure ..................... 5

1.3.2 Metal Complexes . . . . . . . . . . . . . . . . . . . 6

1.4 Iron Proteins . . . . . . . . . . . . . . . . . . . 7

1.4.1 Heme Iron Proteins . . . . . . . . . . . . . . . . . . . . 8

1.4.2 Non-heme Diiron Proteins . . . . . . . . . . . . . . . . . . 10

2 Objective 13

3 A New Pyrrole/Pyrazole Building Block $\quad 15$

4 New Siamese-Twin Porphyrins $\quad 21$

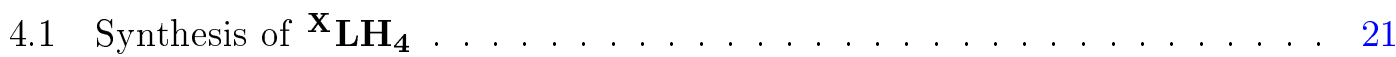

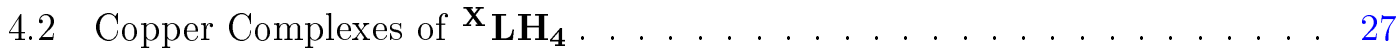

5 Iron Complexes of the Siamese-Twin Porphyrin 33

5.1 Iron Nickel Complexes of the Siamese-Twin Porphyrin . . . . . . . . . . . 33

5.1 .1 Synthesis of $\mathbf{L N i F e C l} \ldots \ldots \ldots$. . . . . . . . . . . . . 34

5.1 .2 Characterization of $\mathbf{L N i F e C l ~ \ldots . . . . . . . . . . . . . ~ . ~} 35$

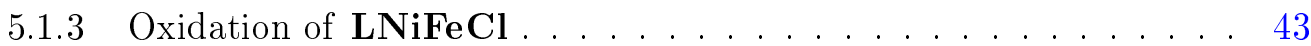

5.1 .4 Reduction of $\mathbf{L N i F e C l} \ldots \ldots \ldots \ldots$. . . . . . . . . 46

5.1 .5 Synthesis of ferrous $\mathbf{L N i F e} \ldots \ldots \ldots \ldots . \ldots . . \ldots 50$

5.2 Mononuclear Iron Complex $\mathbf{L H}_{2} \mathbf{F e C l} \ldots \ldots \ldots \ldots$

5.3 Iron Copper Complex $\mathbf{~ C u F e C l ~ \ldots . ~ . ~ . ~ . ~ . ~ . ~ . ~ . ~ . ~ . ~ . ~ . ~ . ~ . ~ . ~ . ~ . ~} 54$

5.3 .1 Synthesis of $\mathbf{L C u F e C l} \ldots \ldots \ldots \ldots \ldots$

5.3 .2 Characterization of $\mathbf{~} \mathbf{C} \mathbf{C u F e C l} \ldots \ldots \ldots \ldots \ldots \ldots$

5.4 Diiron Complex $\mathbf{L}\{\mathbf{F e C l}\}_{2} \ldots \ldots \ldots \ldots \ldots$

5.4 .1 Synthesis of $\mathbf{L}\{\mathbf{F e C l}\}_{2} \ldots \ldots \ldots \ldots \ldots$

5.4 .2 Characterization of $\mathbf{L}\{\mathbf{F e C l}\}_{2} \ldots \ldots \ldots \ldots$ 
6 Cobalt Complexes of the Siamese-Twin Porphyrin 65

6.1 Nickel Cobalt Complex LNiCo . . . . . . . . . . . . . . . . . . . . 65

6.1 .1 Synthesis of LNiCo . . . . . . . . . . . . . 65

6.1 .2 Characterization of LNiCo . . . . . . . . . . . . 66

6.2 Dicobalt Complex $\mathbf{L C o}_{2} \ldots \ldots \ldots \ldots$. . . . . . . . . . . . . . 69

6.2.1 Synthesis of $\mathbf{L C o}_{2} \ldots \ldots \ldots \ldots$

6.2.2 Characterization of $\mathbf{L C o}_{2} \ldots \ldots \ldots \ldots$

7 Preliminary Reactivity Studies of LNiFe and LNiFeCl 71

8 Summary and Outlook $\quad 75$

9 Experimental Section $\quad \mathbf{7 9}$

9.1 Instruments and Materials . . . . . . . . . . . . . . . . . . . 79

9.2 Synthesis of the Pyrazole Building Blocks 18, 19, 20, 21 and $22 \ldots . .82$

9.2.1 1-(Methoxymethyl)-1H-pyrazole-3,5-dicarbaldehyde (19) . . . . . 82

9.2.2 1-(Methoxymethyl)-3,5-bis(1-hydroxy-3-phenylprop-2-yn-2-yl)$1 H$-pyrazole $(\mathbf{2 0}) \ldots \ldots \ldots \ldots \ldots$. . . . . . . . . . . . . . . . .

9.2.3 1-(Methoxymethyl)-3,5-bis(1-chloro-3-phenylprop-2-yn-2-yl)-1Hpyrazole $(\mathbf{2 1}) \ldots \ldots \ldots \ldots$. . . . . . . . . . . . . . 84

9.2.4 3,5-Bis(1-hydroxy-3-phenylprop-2-yn-2-yl)-1 $H$-pyrazole (18) . . . 85

9.2.5 3,5-Bis(1-chloro-3-phenylprop-2-yn-2-yl)-1 $H$-pyrazole $(\mathbf{2 2}) \ldots 86$

9.3 Synthesis of Siamese-Twin Porphyrins $\mathbf{x}_{\mathbf{L H}} \mathbf{L H}_{4} \ldots \ldots$. . . . . . . 87

9.3.1 General Procedure for the Synthesis of a Siamese-Twin Porphyrino-

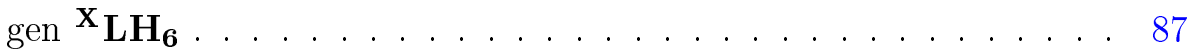

9.3.2 General Procedure for the Synthesis of Siamese-Twin Porphyrins

$\mathrm{x}_{\mathbf{L H}_{4}} \ldots \ldots \ldots \ldots \ldots \ldots$

9.4 Complex Synthesis . . . . . . . . . . . . . . . . . . . . . 95

9.4.1 General Procedure for the Synthesis of Copper Complexes ${ }^{\mathbf{X}} \mathbf{L} \mathbf{C} \mathbf{u}_{\mathbf{2}} \quad 95$

9.4 .2 Iron(III) Nickel(II) Complex LNiFeCl . . . . . . . . . . . . . . . 97

9.4.3 Iron(II) Nickel(II) Complex LNiFe . . . . . . . . . . . . . . . . 98

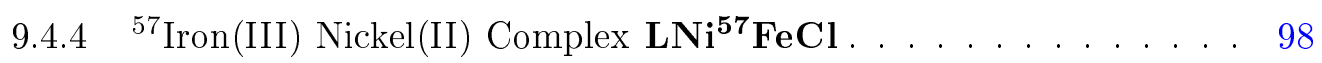

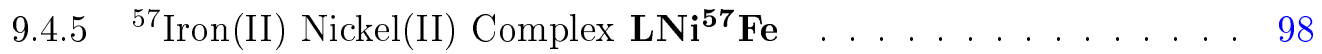

9.4.6 Monoiron Complex of the Siamese-Twin Porphyrin $\mathbf{L H}_{\mathbf{2}} \mathbf{F e}$. . . . 99

9.4 .7 Iron(III) Copper(II) Complex LCuFeCl . . . . . . . . . . . . . . . 99

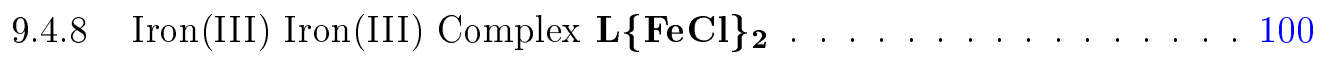

9.4 .9 Nickel(II) Cobalt(III) Complex $\mathbf{L N i C o}^{+} \ldots \ldots$. . . . . . . . . . . 101

9.4.10 Cobalt(III) Cobalt(III) Complex $\mathbf{L C o}_{2}{ }^{2+} \ldots \ldots$. . . . . . . . . 102

$\begin{array}{ll}\text { Appendix } & 103\end{array}$

Crystallographic Data . . . . . . . . . . . . . . . . . . . . . . 103

Additional Spectroscopic Data of $\mathbf{x}_{\mathbf{L H}} \mathbf{H}_{4} \ldots \ldots$. . . . . . . . . . . 108

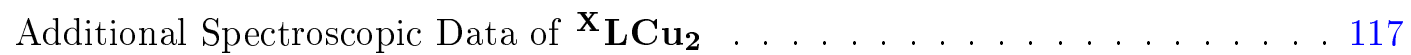


Additional Spectroscopic Data of LNiFeCl . . . . . . . . . . . . . . . . . 121

Additional Spectroscopic Data of $\mathbf{L C u F e C l}$ and $\mathbf{L}\{\mathbf{F e C l}\}_{2} \ldots \ldots . . \ldots 123$

$\begin{array}{lr}\text { Abbreviations } & 125\end{array}$

$\begin{array}{lr}\text { Bibliography } & 127\end{array}$

$\begin{array}{ll}\text { Acknowledgement } & 137\end{array}$ 



\section{General Introduction}

Porphyrins are ubiquitous macrocycles in nature and their metal complexes are involved in multiple key processes in living organisms, such as oxygen transport and photosynthesis. ${ }^{[1-3]}$ Due to their flexible electronic structure, which depends on the substitution pattern, they easily incorporate different metal ions, preferebly in the oxidation state + II. ${ }^{[3,4]}$ Numerous enzymes responsible for either the transport or the conversion of oxygen involve an iron porphyrin complex, called heme, in the active center. ${ }^{[5,6]}$

Whereas nature is able to activate and transfer oxygen to substrates at ambient temperature and pressure, industrial processes usually require harsh reaction conditions. ${ }^{\text {[7] }}$ Thus, detailed investigation into dioxygen activation processes and oxygen transfer in metalloenzymes is of considerable interest. Hence, often low molecular weight analogues are developed to study and structurally understand intermediates in an enzyme's catalytic cycle or as functional models to perform oxygenation catalysis. ${ }^{[8,9]}$

In the present work, a new class of iron porphyrin complexes, the complexes of the Siamese-twin porphyrin, ${ }^{[10]}$ are established. These complexes are inspired by the bimetallic active center of the methane monooxygenase enzyme familiy and the mechanism of cytochrome $\mathrm{P} 450$ to combine their electronic advantages for oxygen activation and binding.

\subsection{Porphyrins}

Porphyrins (2) (Fig. 1.1) are planar, tetrapyrrolic, aromatic macrocycles, which consist of four pyrrole units (1) that are connected in 2- and 5-positions via methine groups (mesopositions). To label the different positions in a heterocyclic ring and the porphyrin the IUPAC nomenclature with arabic numbers is not consequently used in literature. Alternatively, the use of the historical numbering with greek letters is very common and also used in this work. ${ }^{[11]}$

18 of the $22 \pi$-electrons of the porphyrin ring are at once part of the delocalized aromatic system, following Hückel's rule for aromaticity $(4 \mathrm{n}+2 \pi$-electrons, here: $\mathrm{n}=4) .{ }^{[12]}$ One possible conjugation pathway is highlighted in Figure 1.1.

Due to their extended $\pi$-system, as a result of the numerous conjugated double bonds, the HOMO (highest occupied molecular orbital) - LUMO (lowest unoccupied molecular orbital) gap is diminished. Thus, the UV-vis spectra of porphyrins show an intense 


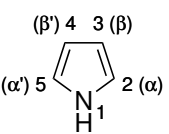

1

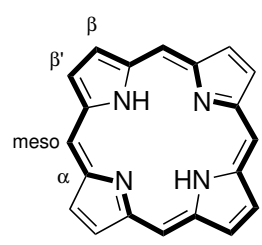

2

Figure 1.1: Left: Pyrrole labeled according to the IUPAC (numbers) and the historical nomenclature (greek letters). Right: Chemical structure of the porphyrin with highlighted $18 \pi$-electron conjugation pathway as "internal cross" and the definition of the positions in the macrocycle.

absorption band (Soret band) at around $400 \mathrm{~nm}\left(\pi-\pi^{*}\right.$ transition) with extinction coefficients of $10^{5} \mathrm{M}^{-1} \mathrm{~cm}^{-1}$ and weak so called Q-bands at wavelengths of 500 to $750 \mathrm{~nm},{ }^{[13,14]}$ resulting in an intensively colored molecule. The UV-vis spectrum of a porphyrin is very sensitive towards minor changes of the electronic structure or the incorporated metal ion and thus is the method of choice for the analysis of porphyrin systems.

When deprotonated, porphyrins are dianionic, tetradentate, chelating ligands with a square planar nitrogen based coordination cavity (diameter: 0.6-0.7 $\AA$ ). ${ }^{[3]}$ Due to their dianionic character porphyrins preferably coordinate dicationic metal ions but also metal ions in lower or higher oxidation states, depending on additional axial ligands and the size of the central metal ion. ${ }^{[15-17]}$ The incoorporation of the metal ion inside the coordination pocket of the porphyrins as out-of plane or in-plane, as shown in Figure 1.2, is controlled by the size of the metal ion, whereby larger metal ions tend to be located out-of plane. Varying the oxidation state of the metal ion changes its size and thus the geometry of the complex.

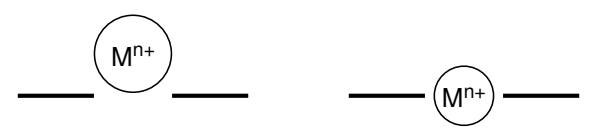

Figure 1.2: Schematic representation of the out-of-plane (left) and in-plane (right) coordination of a metal ion in a porphyrin scaffold. ${ }^{[3,4]}$

\subsection{Expanded Porphyrins}

Expanded porphyrins (EP) are porphyrinoids that contain more than 18 aromatic $\pi$ electrons or more than four heterocyclic building blocks. Due to their red-shifted NIR absorption spectra, their ability to coordinate simultaneously up to two metal ions, and their conformational flexibility affecting macrocycle $\pi$-conjugation and aromaticity, expanded porphyrins are of high interest. A variety of different EPs has been synthesized in the last decades. ${ }^{[18-22]}$ Due to their unique coordination motif these expanded porphyrins are attractive molecules for non-linear optical materials, ${ }^{[23]}$ as funtional dyes, ${ }^{[20,24]}$ ion binding, ${ }^{[25]}$ and the research on aromaticity. ${ }^{[26]}$ 


\subsubsection{Structure and Nomenclature}

The heterocycles within an expanded porphyrin are not limited to pyrrole but can be replaced by several other like thiophene, selenophene or furan. Even silicium-based heterocycles are possible. ${ }^{[27-30]}$ EPs can adopt two coformations. In the normal conformation, all heteroatoms point inwards like in $\mathbf{3}$ and the inverted conformation where at least one heteroatom is pointing outwards (4). The conformation depends on the size of the EP and the substitution pattern in $\beta$ - and meso-positions.

Due to historical reasons expanded porphyrins are often named after their color, like the dark blue Sapphyrin (3) (Fig. 1.3). These common names are still in use among porphyrin chemists, even though there is a systematic nomenclature, following the nomenclature of FRANCK with sligth modifications: ${ }^{[31,32]}$ The EP is mainly named after the number of connected heterocycles $n$ in its inner core: $n=5$ pentaphyrin, $n=6$ hexaphyrin, $n=7$ heptaphyrin, $n=8$ octaphyrin etc.. ${ }^{[3]}$ The number of meso-carbon atoms between each consecutive heterocycle is reflected by the numbers in brackets, seperated by dots. Finally the number of $\pi$-electrons within the conjugation pathway is given in square brackets in front of the name. For instance the complete name of Sapphyrin (3) is [22]pentaphyrin(1.1.1.1.0).

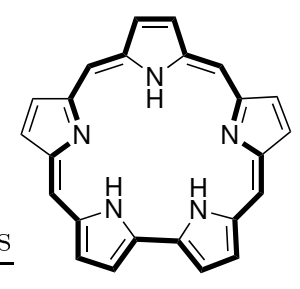

3

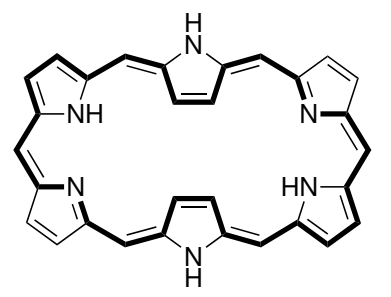

4

Figure 1.3: Structure of [22]pentaphyrin(1.1.1.1.0) (3) and [28]hexaphyrin(1.1.1.1.1.1) (4) each with highlighted $\pi$-electron conjugation pathway.

\subsubsection{Metal Complexes}

A variety of mono- and bimetallic complexes of expanded porphyrins have been synthesized so far. ${ }^{[8,34-36]}$ Due to possible cooperativity between the two metal ions these complexes are highly attractive for reactivity studies. They can act as dinuclear centers for bioinspired catalytic reactions or as molecules for optical data storage. ${ }^{[37]}$ Due to their ability of coordinating multiple metal ions paired with their tunable physical and spectroscopic properties, expanded porphyrin metal complexes have shown their potential as photosensitizer in photodynamic therapy $(\mathrm{PDT})^{[38]}$, as non-linear optical materials ${ }^{[23]}$ and contrast agents for magnetic resonance imaging (MRI). ${ }^{[39-41]}$ 
The flexibility of the EPs increases with their ring size and so does the ability to coordinate metal ions with increasing radii such as lanthanides and actinides. ${ }^{[37,42]}$ In bimetallic complexes the selective incorporation of two equal or different metal ions is also possible, which was demonstrated by OsukA et al. for [26]hexaphyrin(1.1.1.1.1.1) 5 in 2005. ${ }^{[34]}$ Depending on the metal ion and the complexation conditions the donorset of $\mathbf{5}$ can vary from $\mathrm{N}_{3}$ over $\mathrm{N}_{2} \mathrm{C}(7)$ and $\mathrm{N}_{3} \mathrm{C}$ up to $\mathrm{N}_{2} \mathrm{C}_{2}(6,8)$ (Fig. 1.4) and the conformation can be normal or inverted. ${ }^{[20,34-36,43]}$

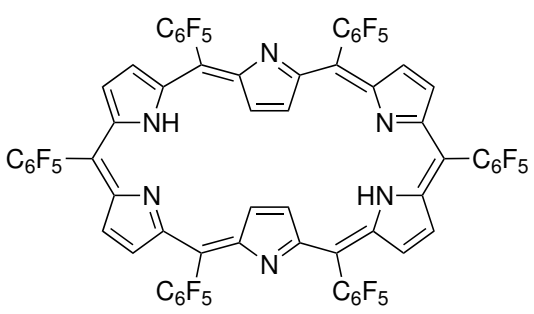

5

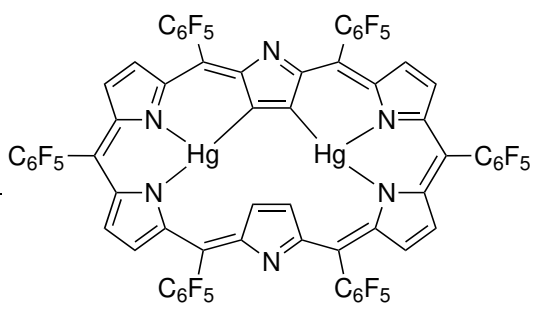

7

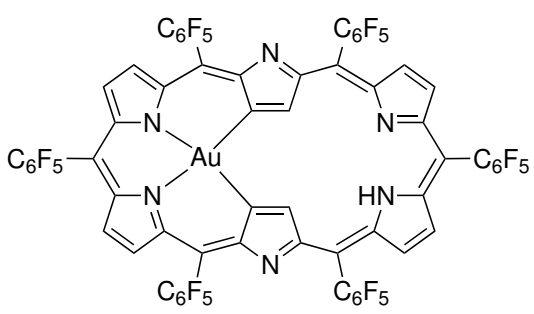

6

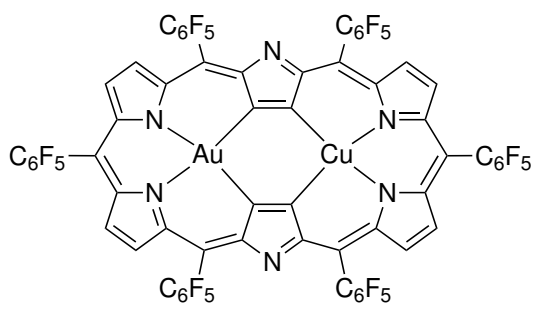

8

Figure 1.4: Selection of structures of metal complexes of the [26] hexaphyrin(1.1.1.1.1.1) $\mathbf{5}$ with different donorsets. ${ }^{[34-36]}$

Due to invertion of one or two pyrrole rings in most metal complexes of hexaphyrins, the classical all-nitrogen coordination motif of a porphyrin can not be preserved. Furthermore, no iron complexes of a hexaphyrin, made of regular pyrrole rings, have been synthesized so far.

\subsection{The Siamese-Twin Porphyrin (STP)}

Even though a lot of different heterocycles were established in expanded porphyrin chemistry, no normal EP bearing a pyrazole was known until 2011, when FRENSCH synthesized the so called Siamese-twin porphyrin (STP) $\left(\mathbf{9}=\mathbf{L H}_{\mathbf{4}}\right)$ (Fig. 1.5). ${ }^{[10]}$ The name originates from its two identical fused porphyrin-like binding pockets that are linked through pyrazole moieties. ${ }^{[10]}$ This non-planar and non-macrocycle-aromatic molecule is characterized by the presence of two binding pockets whose $\mathrm{N}_{4}$-coordination mean planes are nearly orthogonal to each other. 


\subsubsection{Structure}

The structural motif (di-para-pyrazole-[26]hexaphyrin(1.1.1.1.1.1) 10) (Fig. 1.5) of the Siamese-twin porphyrin 9 was formely termed "double porphyrin" and initially, theoretically described by LiND in 1987 but has not been synthesized to date. ${ }^{[4]}$ Since functionalized pyrazoles had proven to be suitable bridging ligands for multi nuclear transition metal complexes, ${ }^{[45]}$ Katsiaouni tried to synthesize the macrocycle 11, which is alkylated in the pyrrole's $\beta$-position, in another attempt to build a "double porphyrin".[46,47]

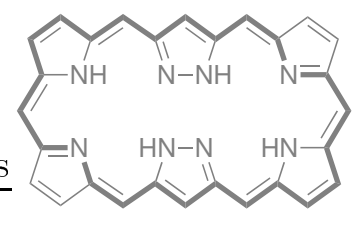

10

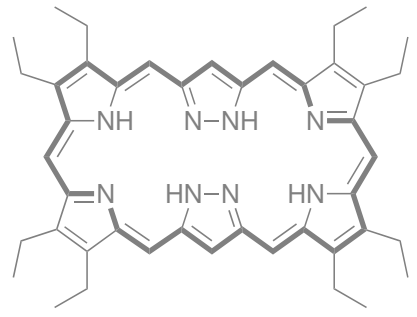

11

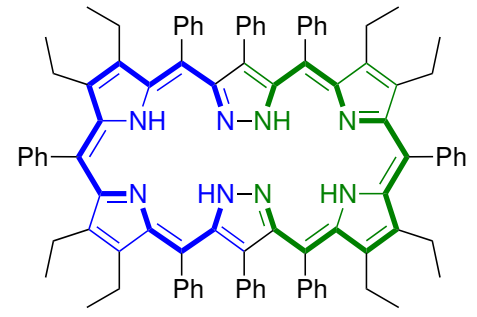

9

Figure 1.5: Suggested structure of di-para-pyrazole-[26]hexaphyrin(1.1.1.1.1.1) (10) (left) and the alkylated double porphyrin $\mathbf{1 1}$ (middle) and synthesized Siamese-twin porphyrin $\mathbf{9}$ (right), each with highlighted $\pi$-electron conjugation pathway.

The precursor porphyrinogen of $\mathbf{1 1}$ was only detected by mass spectrometry (MS), because NMR spectroscopic investigations were not suitable, due to the enormous amount of stereoisomers and the conformational flexibility of the porphyrinogen, but has not been isolated. Furthermore, an oxidation of the pyrazols' meso-positions of the porphyrinogen towards the final "double porphyrin" $\mathbf{1 1}$ was not possible, ${ }^{[48]}$ because these positions were too electron poor, like observed by LASH during the synthesis of carbaporphyrins. ${ }^{49]}$

The synthesis of the new pyrazole building block $\mathbf{1 2}{ }^{[50]}$ allowed for the synthesis of the pyrrole/pyrazole building block $\mathbf{1 3}$, which is the key compound for the synthesis of the STP 9. ${ }^{[10]}$

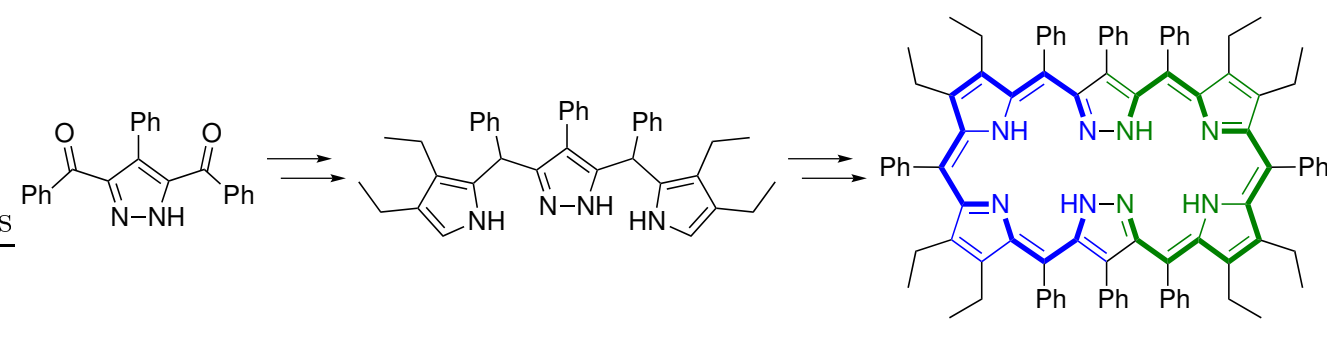

12

13

9

Scheme 1.1: Key compounds $\mathbf{1 2}$ and $\mathbf{1 3}$ for the synthesis of the Siamese-twin porphyrin $\mathbf{9}$.

All peripheral positions of the macrocycle $\mathbf{9}$ were substituted by either ethyl groups (pyrrole $\beta$-positions) or phenyl groups (meso-positions and 4-position of the pyrazole). The 
choice of the substituents and the particular substituent pattern was guided by practical synthesis considerations and to induce the proper conformation of the precursor Siamese-twin porphyrinogen to allow its oxidation to the final product $\mathbf{9}$. The symmetric 3,4-diethylpyrrole is readily accessible ${ }^{[51]}$ and does not give rise to the formation of regioisomers due to the protected 3- and 4-positons. Furthermore the bulky phenyl and ethyl substituents prevented an inverted conformation of the STP.

For electronic reasons the meso-phenyl groups adjacent to the pyrazole were necessary for the oxidation to take place in this positions, due to the phenyl group's electron pushing effect and $\pi$-conjugation. ${ }^{[10]}$

The high steric pressure of the phenyl groups in the STP's backbone induced the unique twisted conformation of the STP (Fig. 1.6), resulting in a non-macrocycle-aromatic molecule, as observed in NMR spectroscopy. ${ }^{[10]}$ Therefore the name "porphyrin" only described the formal fully conjugated system of the STP but not its electronic structure. Further two- or four-electron oxidation of $\mathbf{9}$ by Vogel in 2016 did not lead to a macrocycle-aromatic molecule either. Even though NMR and UV-vis spectroscopy could show a highly delocalized extended $\pi$-system, no switch to a macrocycle-aromatic molecule was observed. ${ }^{[52]}$ A switch was expected because it was observed in hexaphyrin analogues, which normally switch between Hückel and Möbius aromatic systems, depending on their redox and protonation state. ${ }^{[2,26]}$ Instead, upon oxidation a linkage of one pyrazole nitrogen atom and the meso-phenyl group for each two-electron oxidation was observed (Fig. 1.6).

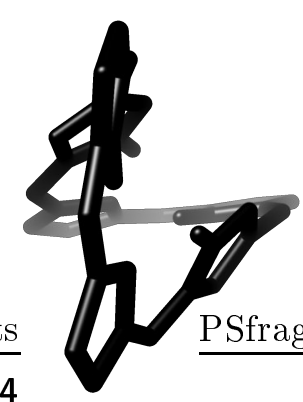

(a)

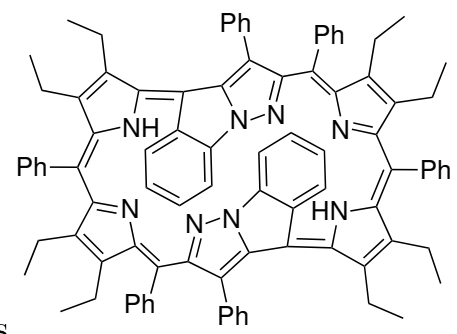

14

(b)

Figure 1.6: (a) Sideview of the core structure of 9. (b) Linkage product (14) of the fourelectron oxidation of $\mathbf{9}$.

Despite its non-macrocyle-aromaticity the Siamese-twin porphyrin is a highly interesting molecule for dinuclear metal complexes, due to its unique coordination motif.

\subsubsection{Metal Complexes}

With the STP $\left(\mathbf{L H}_{4}\right)$ several homo- and hetero-bimetallic complexes LX (Fig. 1.7) were synthesized. An enormous influence of the central metal ions on the electronic properties of the macrocycle could be demonstrated by means of, e.g., cyclic voltammetry and EPR 
spectroscopy. ${ }^{[10,53-55]}$ Interestingly enough, the mono-nickel complex $\mathbf{L H}_{\mathbf{2}} \mathbf{N i}$ could be synthesized selectively at ambient temperature, making heterobimetallic complexes like LNiCu accessible. ${ }^{[55]}$

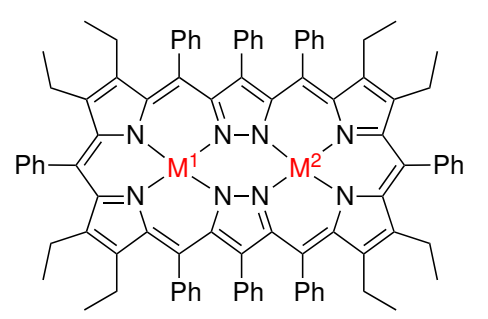

$$
\begin{array}{ll}
\mathbf{L H}_{\mathbf{2}} \mathbf{N i}: & \mathrm{M}^{1}=2 \mathrm{H}, \mathrm{M}^{2}=\mathrm{Ni} \\
\mathbf{L N i C u}: & \mathrm{M}^{1}=\mathrm{Cu}, \mathrm{M}^{2}=\mathrm{Ni} \\
\mathbf{L N i}_{\mathbf{2}}: & \mathrm{M}^{1}=\mathrm{M}^{2}=\mathrm{Ni} \\
\mathbf{L C u}_{\mathbf{2}}: & \mathrm{M}^{1}=\mathrm{M}^{2}=\mathrm{Cu}
\end{array}
$$

Figure 1.7: Homo- and heterobimetallic complexes of the STP $\mathbf{L H}_{4} \cdot{ }^{[10,53-55]}$

The metal complexes $\mathbf{L X}$ exhibited well-defined redox processes in their cyclic voltammogram, whose first two oxidations were shown to mainly take place at the dipyrromethane subunits of the organic macrocycle (Fig. 1.8). ${ }^{[53]}$ Thus the Siamese-twin porphyrin is a non-innocent ligand.

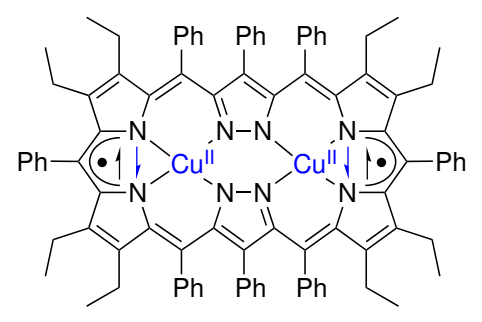

Figure 1.8: Location of the ligand oxidation of $\mathbf{L C \mathbf { C u } _ { 2 }}$. ${ }^{[53]}$

The oxidation potentials change remarkably when one or two metal ions are changed from copper to nickel or vice versa. With a shift of up to $260 \mathrm{mV}$, the influence of the metal ion is much higher than in the corresponding meso-tetraphenylporphyrinato(TPP)-complexes $(\Delta \mathrm{E}=60 \mathrm{mV}){ }^{[56-58]}$ This underlines the principally different electronic structures of the non-macrocycle-aromatic STP compared to the aromatic porphyrins even further.

Remarkably $\mathbf{L} \mathbf{C u}_{2}$ showed a ferromagnetic coupling between two pyrazolato bridged copper(II) ions observed for the first time, due to orthogonal coordination pockets. ${ }^{\text {[53] }}$

\subsection{Iron Proteins}

Numerous metalloproteins that are responsible for either the transport or the conversion of dioxygen involve an iron ion in the active center. ${ }^{[6]}$ They can be distinguished between three classes, heme, non-heme diiron centers, and mononuclear non-heme iron centers (Scheme 1.2), whereas only the non-heme diiron and heme iron centers will be discussed in more detail in this chapter. Heme proteins possess a substituted porphyrin ring with an incoorporated iron ion, which will not described in more detail. 


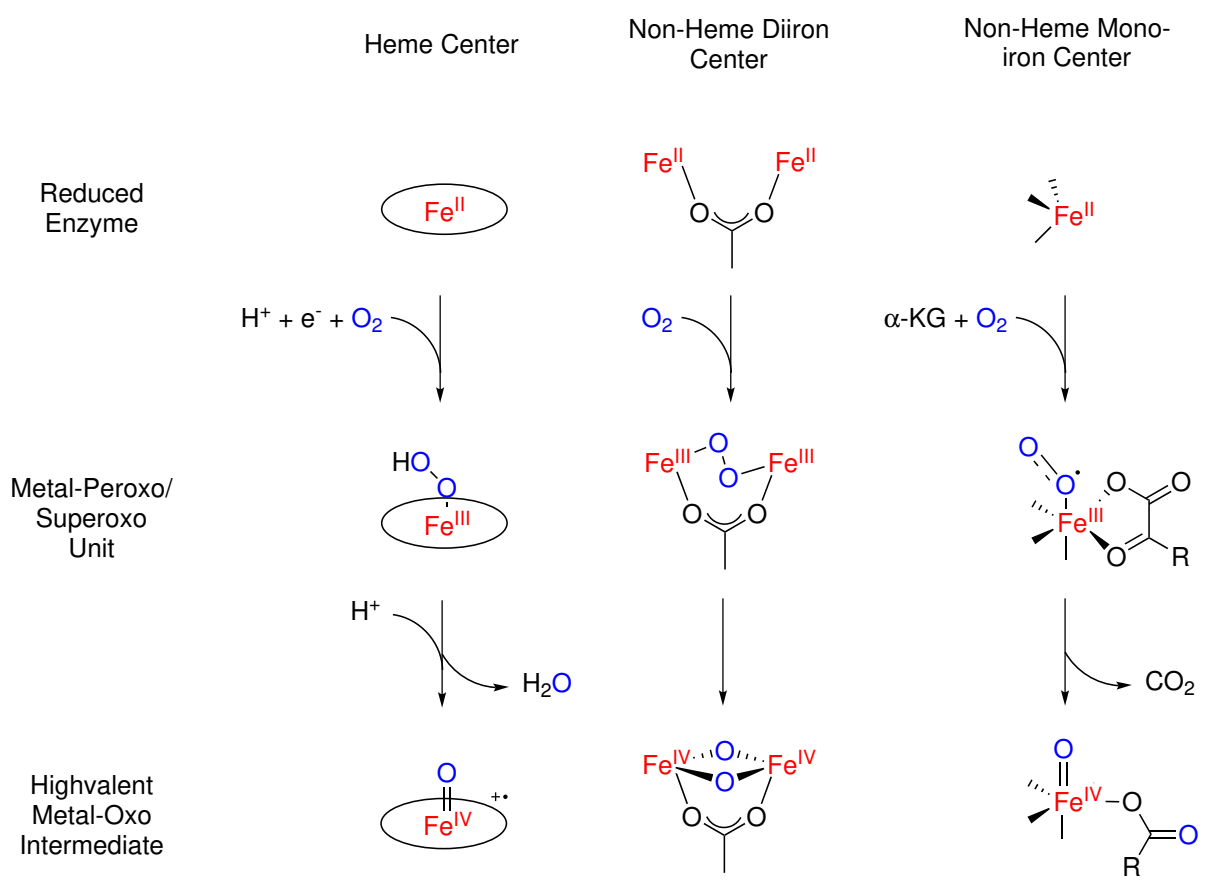

Scheme 1.2: Schematic representation of dioxygen activation by heme, non-heme diiron centers and non-heme monoiron centers. ${ }^{[59]}$

As can be seen in Fig 1.2, non-heme monoiron centers obtain their four electrons, necessary for the reduction of molecular oxygen, partially from external electron donors. ${ }^{[60]}$ In contrast, in non-heme diiron centers the two iron ions can provide the required four electrons alone. With the coordination of molecular oxygen to the reduced iron(II) form of the active center a diiron(III) peroxo species is formed which transforms into a high valent bis( $\mu$-oxo)-diiron(IV) species, whose coordination geometry is called "diamond core", by reductive homolytic cleavage of the dioxygen bond. This iron(IV) intermediate was identified as the active species. ${ }^{[5,61,62]}$

Two classes of proteins that are able to bind and transfer molecular oxygen to organic substrates with the afore-mentioned mechanisms are the heme based cytochrome P450 (Cyt P450) (Chap. 1.4.1) and the non-heme diiron containing soluble methane monoxygenase (sMMO) (Chap. 1.4.2). ${ }^{[63]}$

\subsubsection{Heme Iron Proteins}

Heme iron proteins contain an iron porphyrin in their active center. Such metalloproteins catalyze a variety of chemical reactions. such as the functionalization of aliphatic $\mathrm{C}-$ $\mathrm{H}$ bonds to alcohols (cytochrome $\mathrm{P} 450$ ) or they operate as oxygen carriers in living organisms (hemoglobin). ${ }^{[5,6]}$ The various heme iron proteins differ only in the protein's environment of the active center and in the proximal and distal ligands. 


\section{Cytochrome P450}

Cytochrome P450, named after its carbon monoxid complex's absorption band at $450 \mathrm{~nm}$, is a monoxygenase. It plays a major role in the biosynthesis of steroids, carcinogenesis and drug metabolism. Cyt $\mathrm{P} 450$ catalyze the hydroxylation of non-activated $\mathrm{C}-\mathrm{H}$ bonds under physiological conditions, a reaction that is highly unspecific with a high activation energy barrier when uncatalyzed. ${ }^{[5,64]}$

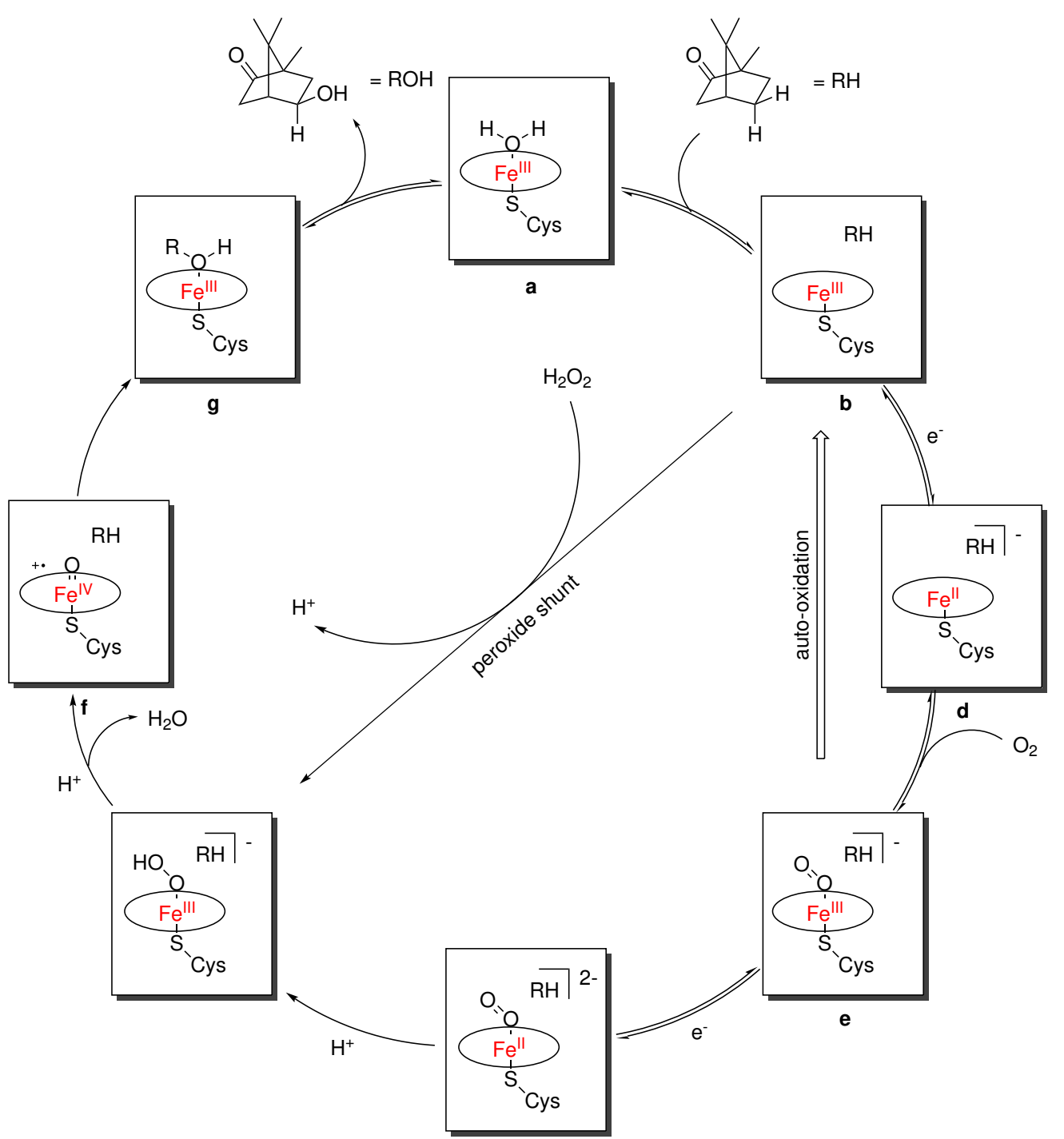

Scheme 1.3: Schematic representation of the catalytic cycle of the hydroxylation of campher by cytochrome P450cam. ${ }^{[64]}$

Cytochrome P450, more precisely Cytochrome P450cam (Cyt P450cam), is a well studied metalloenzyme catalyzing the regio and stereo specific hydroxylation of campher (Scheme 1.3). However, several details of the mechanism of this hydroxylation (Scheme 1.3) are still unexplained. ${ }^{[5,64]}$ Whereas the intermediates $\mathbf{a}, \mathbf{b}$ and $\mathbf{g}$ have been structurally characterized, most other intermediates were postulated through spectroscopic comparison with model complexes. One reason is the limited lifetime of 
high valent iron oxo species even at low temperatures. Intermediate f, often labeled as Compound I (Cpd I) or "active oxygen species", is the key intermediate for the activation of molecular oxygen. ${ }^{[3,5,64-67]}$

As can be seen in Scheme 1.3 two of the four electrons necessary are provided by the substrate, whereas the remaining two reduction equivalents are provided by external donors. Hence, it is clear that mononuclear heme iron complexes are not able to activate or split molecular oxygen by themselves. Only the interaction with the enzyme framework allows this reactivity, ${ }^{[68]}$ showing the importance of the latter in enzymatic catalysis. Thus, the enzyme framework does not only ensure the spacial proximity between substrate and active center but rather protects it against undesired side reactions and provides the channels for the reactants, including electrons and protons. ${ }^{[68]}$

\subsubsection{Non-heme Diiron Proteins}

All non-heme diiron enzymes possess a similar coordination environment of their iron center with carboxylic aminoacids i.e., glutamate and aspartate, ${ }^{[69]}$ resulting in an extremely flexible conformation during dioxygen activation. ${ }^{[0,71]}$ Besides a few exceptions, for example Hemerythrin, the main function of these iron proteins is the activation of molecular oxygen and the subsequent oxygenation catalysis of various substrates. ${ }^{[69]}$ The main pathway of this activation reaction is displayed in Figure 1.2.

\section{Methane Monoxygenase}

The methane monoxygenase (MMO) family is another representative of non-heme diiron enzymes. It is an outstanding family of enzymes from methanotropic bacteria, using methane as carbon and energy source by the oxidation to methanol. ${ }^{[61,63,72]}$ The first step of the methane metabolism is the oxidation of the robust $\mathrm{C}-\mathrm{H}$ bond, a tremendous performance. ${ }^{[63]}$ The industrial oxidation of methane to methanol is thereby only accessible via a two-step process, which makes the process unselective and cost-intensive. ${ }^{[7,73]}$

In general, two types of MMOs, the particulate form (pMMO) having copper in its active center and the soluble form (sMMO) with two iron atoms in its active center, can be distinguished. Due to its accessibility, stability and solubility the sMMO is the MMO type of choice for the examination of the dioxygen activation. ${ }^{[63,68]}$ Its hydroxylase subunit $\mathrm{MMOH}$, in which the oxidation of methane takes place, is able to oxidize different saturated and unsaturated, linear, branched or cyclic hydrocarbons up to $\mathrm{C}_{8}$ as well as 
halogenated derivatives in laboratory experiments, if it gets chemically reduced by an external reductant. ${ }^{63,68,74,75]}$

The coordination motif of the sMMO is displayed in Scheme 1.4.
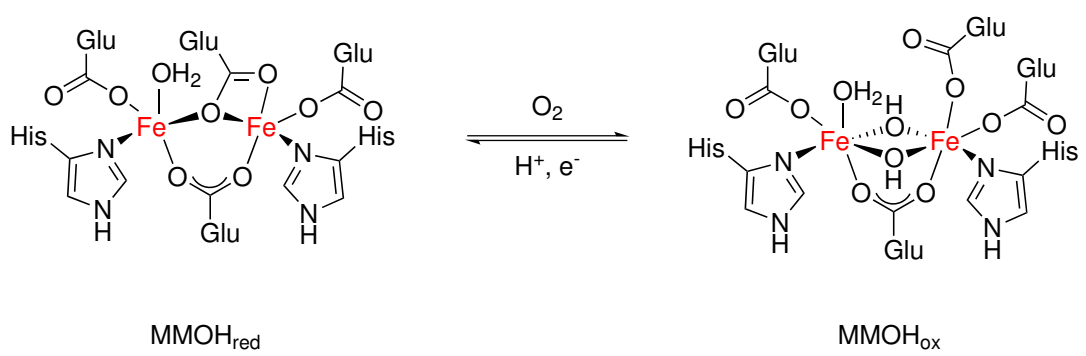

$\mathrm{MMOH}_{\mathrm{Ox}}$

Scheme 1.4: Schematic representation of the carboxylate shift of MMOH in its reduced (left) and oxidized form (right).

Due to the carboxylate shift the bridging glutamate residues can bind either in a $\mu-\eta^{1}: \eta^{2}$ or end-on fashion, depending on the oxidation state of the enzyme. ${ }^{[63,76]}$

Scheme 1.5 shows a schematic representation of the catalytic cycle of the soluble methane monoxygenase hydroxylase (sMMOH) center. Only the reduced and oxidized forms $\mathrm{MMOH}_{\text {red }}$ and $\mathrm{MMOH}_{\mathrm{ox}}$ could be authenticated by solid state crystal structure determination. The other intermediates were characterized or determinated by a variety of spectroscopic techniques like UV-vis and EPR spectroscpocy, stopped-flow measurements, resonance Raman and especially Mößbauer spectroscopy, ${ }^{[63,75,77-81]}$ one of the most precise techniques for the determination of the iron ion's oxidation and spin state. ${ }^{[82]}$

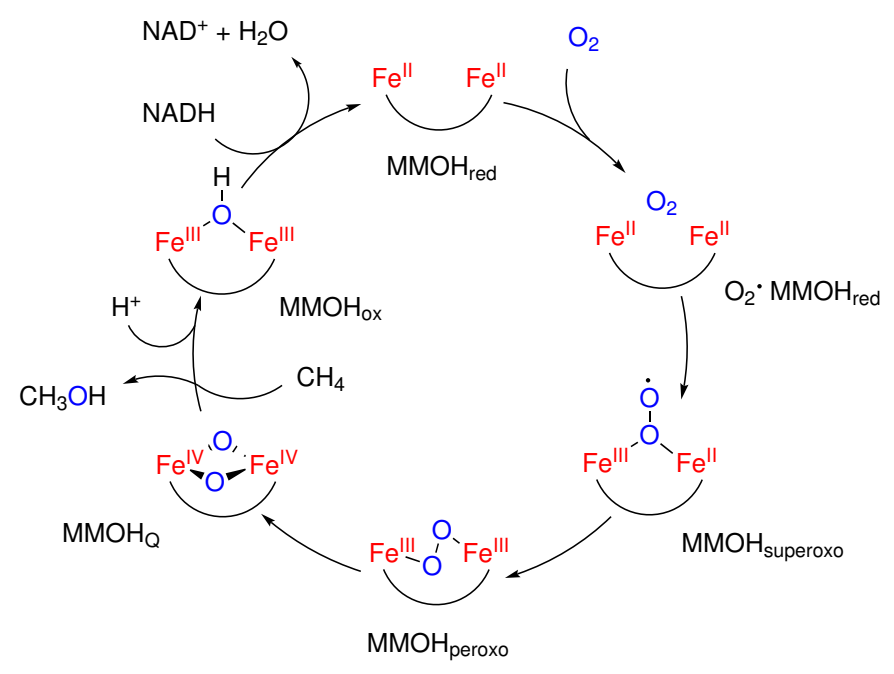

Scheme 1.5: Schematic representation of the catalytic cycle of the soluble methane monoxygenase hydroxylase (sMMOH).

Interestingly enough, the peroxo intermediate $\mathrm{MMOH}_{\text {peroxo }}$ is stable under ambient temperatures in contrast to synthetic peroxo-complexes and does not decompose into a diiron(II) species. ${ }^{[83]}$ Instead, a high valent iron-oxo intermediate $\left(\mathrm{MMOH}_{\mathrm{Q}}\right)$ is formed, 
whose decay to $\mathrm{MMOH}_{\mathrm{ox}}$ can be accelerated by the addition of a substrate. Therefore $\mathrm{MMOH}_{\mathrm{Q}}$ is distinguished to be the active species in the oxidation of methane. ${ }^{[75,79,80]}$ The oxidation state of + IV could be determined by Mößbauer spectroscopy, showing only one iron species with a minor isomer shift of $\delta=0.17 \mathrm{~mm} \cdot \mathrm{s}^{-1}$, a typical value for $\mathrm{Fe}(\mathrm{IV}) .{ }^{[79,82]}$

An oxidation state of + IV was confirmed, among others, by EXAFS measurements. ${ }^{[63]}$ However, the actual structural motif of the iron center could not be identified with absolute certainty, due to the absence of a solid state crystal structure. Furthermore the mechanism of the substrate oxidation is still the subject of ongoing research and various calculations and measurements are proposing and assuming different mechanisms. ${ }^{[84,85]}$ 


\section{Objective}

It has been shown that metalloproteins containing redox active metal ions, for example iron, in their active center efficiently activate and transfer dioxygen to various substrates, which is a four electron redox process, very efficiently at ambient conditions. The familiy of Cyt P450 for instance combines, in the key intermediate Cpd I, a porphyrin cation radical scaffold with a high valent oxoiron(IV) unit to oxygenate various substrates. Furthermore, the Siamese-twin porpyhrin (STP) has been shown to be a suitable platform for hetero- and homobimetallic complexes. Their redox chemistry is fascinating, even though it is much distinct from that of a normal porphyrin scaffold. ${ }^{[52-55,86]}$ With the synthesis of hetero- and homobimetallic complexes of the STP incorporating redox active metal ions like iron, suitable complexes for the activation of small molecules, especially molecular oxygen, can likely be obtained. The complexes combine two functional principles of a mononuclear heme iron enzyme, namely a redox non-innocent oligopyrrole scaffold, and of a non-heme diiron enzyme. In the latter one the two redox active metal ions work in concert to provide the four electrons needed for the activation and conversion of molecular oxygen. The Siamese-twin porphyrin scafffold has the ability to supply up to two electrons, like it has been shown for the nickel(II) and copper(II) complexes. ${ }^{[55,86]}$ Furthermore, in their ferrous state, the two iron ions can potentially provide two electrons each by the oxidation state change to iron(IV). The combination of the STP with two iron(II) ions should therefore be able to supply up to six electrons for the activation of small molecules and should further be of particular interest with regard to its redox chemistry in general.

The aim of this work was to synthesized and characterize first hetero- and homobimetallic iron complexes of the parent STP and to investigate their redox behavior to obtain first insights into the fundamental iron coordination chemistry of the STP scaffold.

With the use of different metal ions within a heterobimetallic complex, iron complexes with characteristic redox potentials can the synthesized. Analytic methods should comprise different techniques like UV-vis, IR, EPR and Mößbauer spectroscopy as well as magnetic susceptibility measurements. 


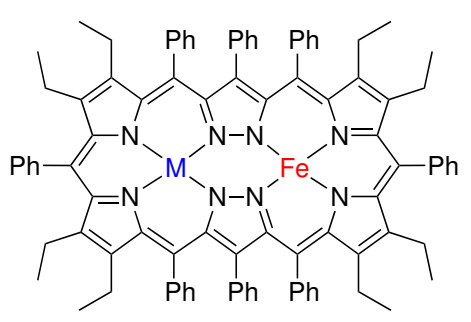

$\mathrm{M}=\mathrm{Fe}, \mathrm{Ni}, \mathrm{Cu}$

Figure 2.1: Potential homo- and heterobimetallic iron complexes of the parent Siamese-twin porphyrin.

Furthermore, new STPs with different substitution patterns should be synthesized, since so far, no structural variations of the parent free base Siamese-twin porphyrin $\left(\mathbf{L H}_{\mathbf{4}}\right)$ have been reported.

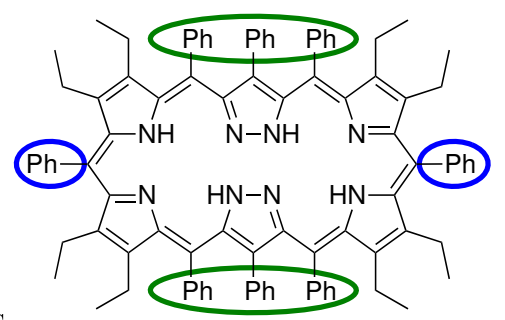

$\mathrm{LH}_{4}$

Figure 2.2: Chemical structure of the free base parent STP $\mathbf{L H}_{4}$ with highlighted substituents in the pyrazoles' meso and 4-position (green) and the meso-phenyl groups located between two pyrrolic moieties (blue).

A variation of the substituent at the pyrazoles' meso- and 4-position (Fig. 2.2 green) would change the molecule's physical properties and should minimize the steric demand in the STP's backbone, which would result in a more planar conformation. Variation of the meso-phenyl group located between two pyrrolic moieties (Fig. 2.2 blue) would primarily change the molecule's electronic and physical properties. 


\section{A New Pyrrole/Pyrazole Building Block}

Since the unique twist of the STP, induced by the high steric demand of the bulky phenyl groups, prevents a macrocycle-aromatic system, a planarization of the whole STP is desirable. A more planar conformation of the Siamese-twin porphyrin would further lead to an opening of the coordination pocket in metal complexes and the corresponding metal ion would be less shielded. Geometry optimizing DFT calculations have shown, that the Siamese-twin porphyrin core would be completely flat, when any substituent is taken away. ${ }^{[54]}$ However, it is more practicable to minimize the steric demand in the backbone of the pyrazole building block 15, since the use of non-substituted pyrrole led to hardly soluble products with vanishingly low yields in their synthesis and the follow up reaction. ${ }^{[86]}$ Nevertheless, electron donating groups in the pyrazole's mesopositions are required so that these positions can be oxidized. ${ }^{[48]}$ Because a synthesis of a pyrazole building block with phenyl groups only in the meso-position (17) (Scheme 3.1), for example by reacting phenyllithium with $1 H$-pyrazole-3,5-dicarbaldehyde (16), was not successful so far, ${ }^{[87]}$ a building block with phenylacetylene groups in the mesopositions (18) was synthesized in this thesis. This compound has a lower steric demand in the backbone but should still be easy to oxidize in the pyrazoles' meso-positions in the corresponding STP, since the phenyl groups still have an electron donating effect via the acetylene linker.

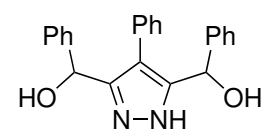

15

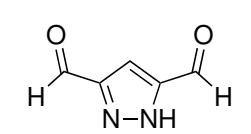

16
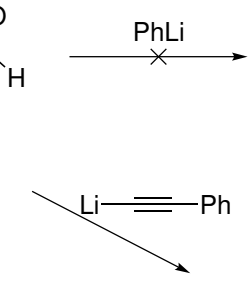

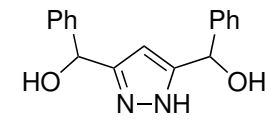

17

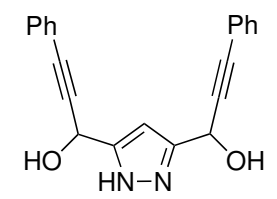

18

Scheme 3.1: Structure of the initially used diol $\mathbf{1 5}$ and the schematic reaction towards new pyrazole builing blocks $\mathbf{1 7}$ and $\mathbf{1 8}$ without any substituent in the pyrazole's 4position, with $1 H$-pyrazole-3,5-dicarbaldehyde (16) as starting point. 
The synthesis of pyrazole-based diol $\mathbf{1 8}$ seemed to be the most challenging step because it was assumed to react similar to the pyrazol building block 15. The meso-position should be more activated towards a nucleophilic attack of the pyrroles' $\alpha$-position and therefore 18 should react more easily with 3,5-diethylpyrrole.

The synthesis was started from $1 H$-pyrazole-3,5-dicarbaldehyde (16), which dimerizes over time leading to a nearly insoluble and unreactive white dimer, ${ }^{[8]}$ which did not further react with lithium phenylacetylide. To increase solubility by preventing the dimerization and to protect the NH-function in further reactions, a methoxymethyl (MOM) protecting group was introduced. The MOM group should be easy to cleave under mildly acidic conditions, for example in the reaction step in which the $\mathrm{OH}$ is exchanged by a chloride by use of $\mathrm{SOCl}_{2}$. Therefore no extra reaction step for the cleavage of the MOM group should be necessary.

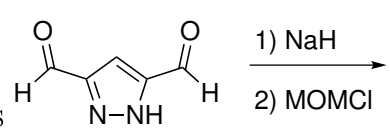

16

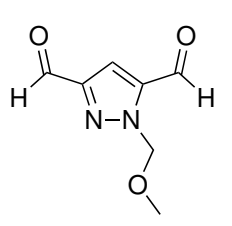

19

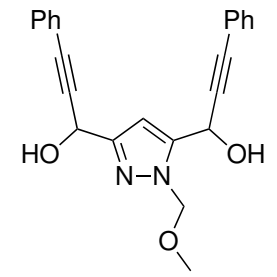

20

Scheme 3.2: Synthesis of 1-(methoxymethyl)-3,5-bis(1-hydroxy-3-phenylprop-2-yn-2-yl)- $1 H$ pyrazole $(\mathbf{2 0})$.

The dicarbaldehyde $\mathbf{1 6}$ was suspended in a large amount of THF over night and the pyrazole was deprotonated with sodium hydride. Subsequently $\mathrm{MOMCl}$ was added and the resulting mixture was stirred for 50 min before the reaction was stopped by the addition of water. After extraction with dichloromethane the raw product was purified by column chromatography to yield the desired product $\mathbf{1 9}(60 \%)$. 19 was characterized via EI mass spectrometry and NMR spectroscopy (see Chap. 9.2.1).

To introduce the phenylacetylene group $n$-butyllithium was added to a solution of phenylacetylene $(\mathrm{PhCCH})$ in dry $\mathrm{THF}$ at $-78{ }^{\circ} \mathrm{C}$ under inert conditions and the mixture was stirred for 1 hour. Subsequently, 19, dissolved in THF, was added and the solution was warmed up to ambient temperature over $1 \mathrm{~h}$. Aqueous ammonium chloride solution was added and the reaction mixture was extracted with THF and washed with brine. After drying the raw product was purified by column chromatography to yield the desired product $(73 \%)$. The diol $\mathbf{2 0}$ was characterized by EI mass spectrometry and NMR spectroscopy (Fig. 3.1).

A reaction of 1-(methoxymethyl)-3,5-bis(1-hydroxy-3-phenylprop-2-yn-2-yl)-1 $H$-pyrazole (20) with $\mathrm{SOCl}_{2}$ according to literature ${ }^{[10]}$ was not successful. ${ }^{1} \mathrm{H}$ NMR spectroscopy of the reaction outcome showed neither the anticipated reaction nor a cleavage of the MOM protecting group. Most of the starting material was still intact, but decomposed with extended reaction time or higher temperatures. Especially the unsuccessful cleavage of 
the protecting group was surprising, because it should be easily split off under already mild acidic conditions ${ }^{[89]}$ and even easier with refluxing $\mathrm{SOCl}_{2}$.

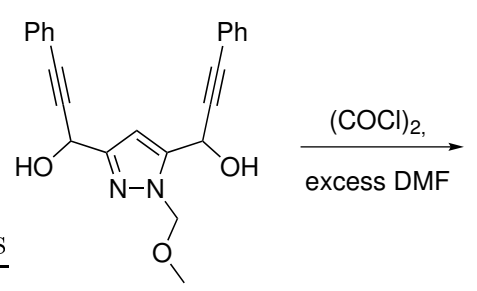

20

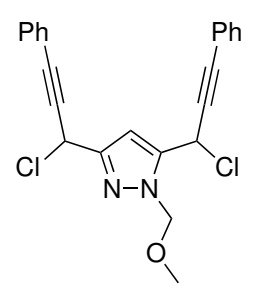

21

Scheme 3.3: Synthesis of 1-(methoxymethyl)-3,5-bis(1-chloro-3-phenylprop-2-yn-2-yl)- $1 H$ pyrazole (21).

The reaction with oxalylchloride $(\mathrm{COCl})_{2}$ instead of thionylchloride did not yield the expected product either, when literature known procedures were used. ${ }^{[90]}$ Only an increase of the amount of dimethylformamide (DMF) to more than 100 equivalents made the conversion to the dichloride compound $\mathbf{2 1}$ possible.

$(\mathrm{COCl})_{2}$ was added to a solution of DMF and acetonitrile at $-20{ }^{\circ} \mathrm{C}$ under inert conditions and the solution was stirred for $20 \mathrm{~min}$. The diol 20, dissolved in MeCN, was subsequently added dropwise and the reaction mixture was allowed to warm up to ambient temperature and stirred for $2.5 \mathrm{~h}$. After removing the solvent the raw product was purified by column chromatography to yield $\mathbf{2 1}(68 \%)$. The methoxy-methyl group was still present, as could be seen in mass spectrometry and NMR spectroscopy by the occurrence of the characteristic signals for the MOM's $\mathrm{CH}_{3}$ and $\mathrm{CH}_{2}$ groups (Fig. 3.1).

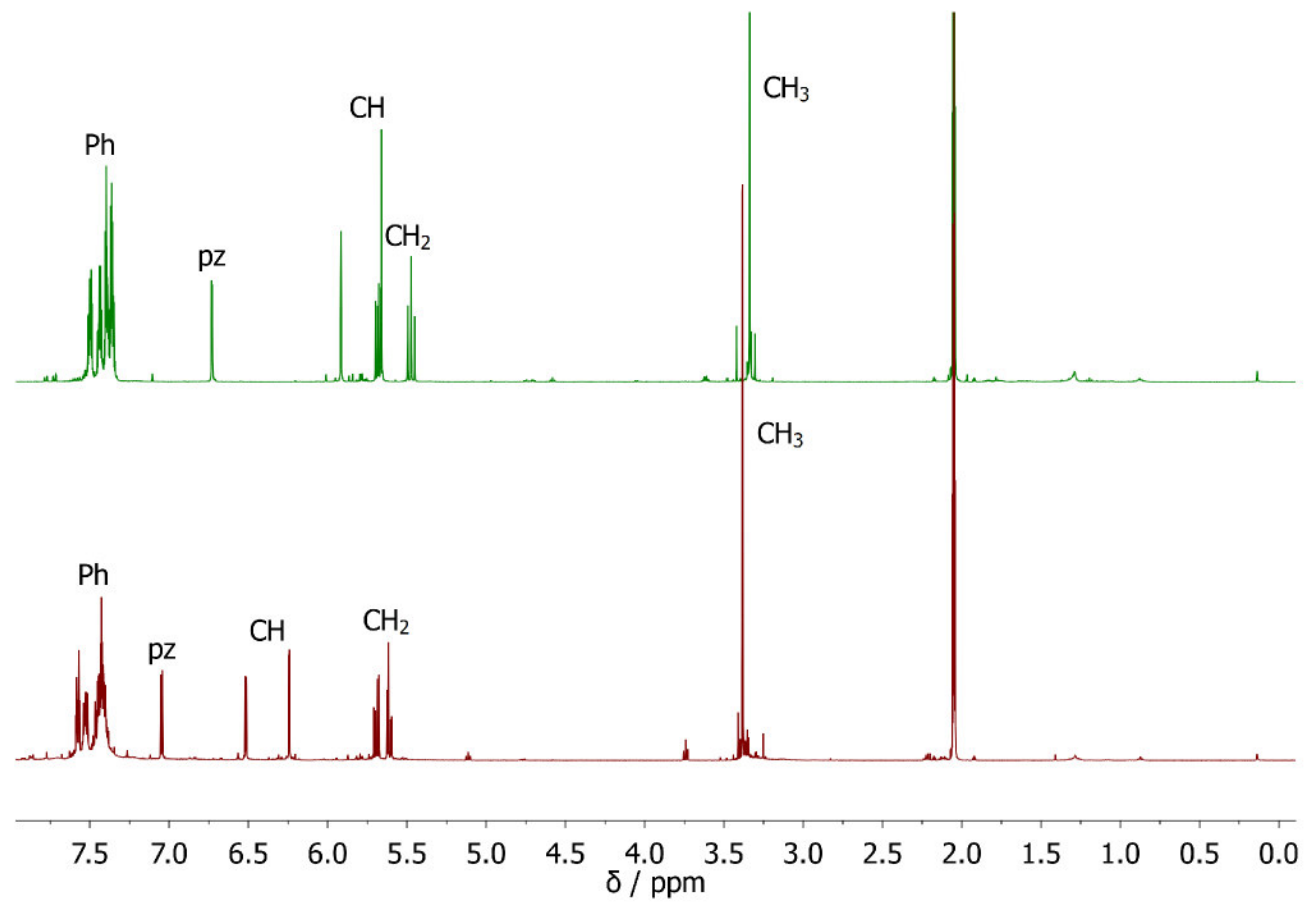

Figure 3.1: ${ }^{1} \mathrm{H}$ NMR spectra of the diol 20 (top) and the corresponding dichloride compound 21 (bottom) in acetone- $\mathrm{d}_{6}$. 
Unfortunately the dichloride compound $\mathbf{2 1}$ showed faster decomposition than the regular dichloride building block with three phenyl groups, but could nevertheless be used for coupling reactions with 3,5-diethylpyrrole. Because the coupling was not successful under the literature known conditions, ${ }^{[10,46]}$ 3,5-diethylpyrrole was replaced by pyrrole, 5 -phenyl-dipyrromethane or thiophene, the latter one being more reactive in $\alpha$-position, and used for coupling reactions with both the dichloride (21) and the diol building block (20) (see Tab. 3.1). None of these reactions led to a coupling.

Furthermore, the MOM group could not be removed, not even under harsh acidic conditions or with heterogeneous catalysts like $\mathrm{KSO}_{4} \cdot \mathrm{SiO}_{2}$ and $\mathrm{ZnBr} .{ }^{[91-94]}$ The presence of the protecting group could lead to problems after the successful coupling with one of the heterocycles, because a ring closing towards the corresponding porphyrinogen would eventually not be possible due to the steric demand of the two MOM groups that are both pointing into the coordination pockets. Furthermore, the MOM group would prevent the corresponding pyrazole nitrogen atom from coordination to metal ions. Therefore a synthesis without a protecting group was developed.

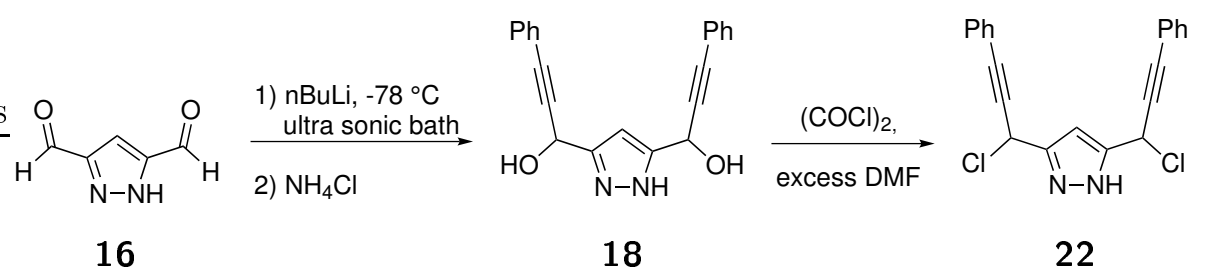

Scheme 3.4: Synthesis of 3,5-bis(1-chloro-3-phenylprop-2-yn-2-yl)-1H-pyrazole (22).

The $1 H$-pyrazole- 3,5 -dicarbaldehyde $\mathbf{1 6}$ was suspended in THF over night in an ultra sonic bath connected to water cooling. $n$-Butyllithium was added to a solution of phenylacetylene in dry $\mathrm{THF}$ at $-78{ }^{\circ} \mathrm{C}$ under inert conditions and the mixture was stirred for $1 \mathrm{~h}$. Subsequently the mixture was slowly added to the suspension of $1 H$-pyrazole-3,5-dicarbaldehyde (16). The mixture was treated in the ultrasonic bath for additional $5 \mathrm{~h}$. Afterwards a saturated aqueous ammonium chloride solution was added and the reaction mixture was extracted with THF and washed with brine. After drying, the crude product was purified by column chromatography and the desired diol 18 was obtained in a lower yield $(26 \%)$ than the protected one (44\% over two steps). 18 was characterized by EI mass spectrometry and NMR spectroscopy (Fig. 3.2).

Like the MOM protected building block 20, reaction with oxalylchloride yielded the dichloride compound $\mathbf{2 2}$ in reasonable quantity $(35 \%)$. The shift of the proton NMR signals in respect to the diol $\mathbf{1 8}$ is not as obvious as is was in case of the MOM protected one, but the $\mathrm{OH}-$ peak was not observable anymore. Nevertheless, the carbon NMR spectrum indicate a shift of the corresponding carbon atom and the meso-pyrazol acetylene carbon signals as expected. 


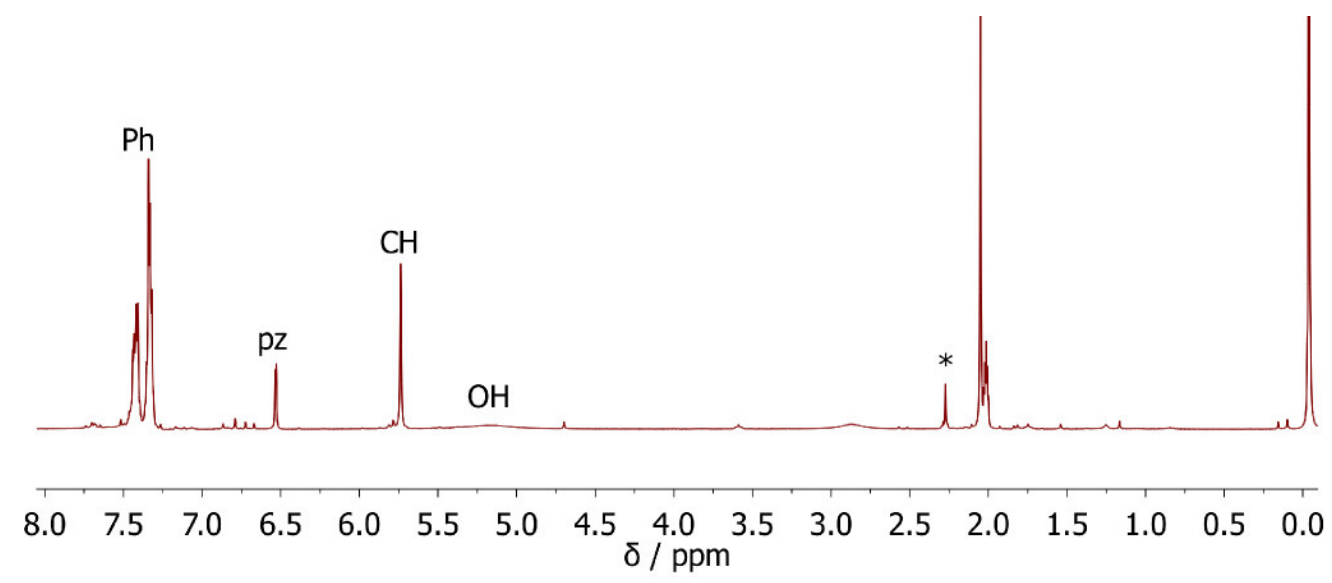

Figure 3.2: ${ }^{1} \mathrm{H}$ NMR spectrum of the diol 18 in acetone- $\mathrm{d}_{6}$. Solvent impurities are marked with an asterisk.
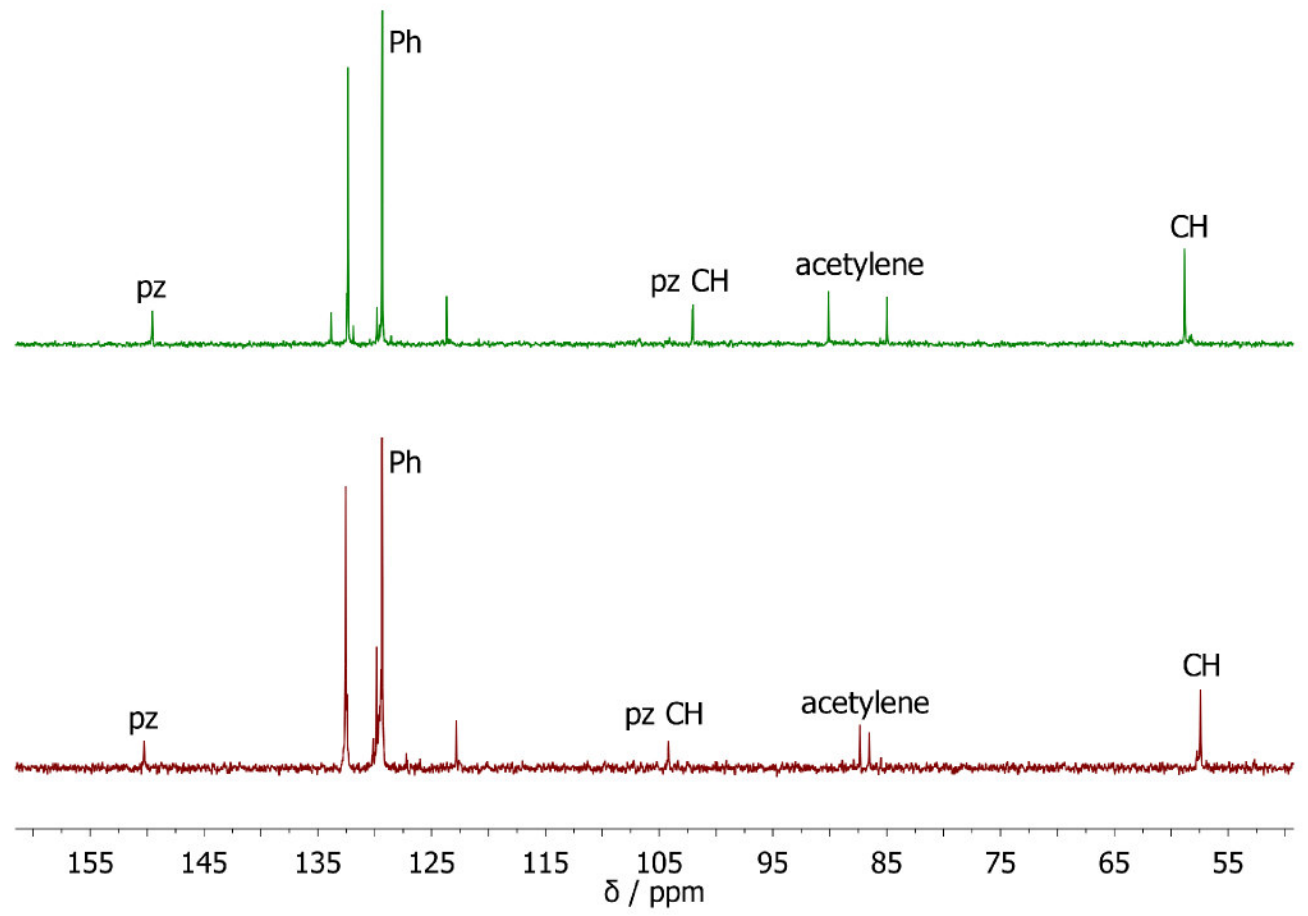

Figure 3.3: Low field region of the ${ }^{13} \mathrm{C}$ NMR spectrum of the diol $\mathbf{1 8}$ (top) and the corresponding dichloride compound 22 (bottom) in acetone- $\mathrm{d}_{6}$. 
The dichloride compound $\mathbf{2 2}$ was decomposing rapidly even under inert conditions and was therefore only used in direct coupling with 3,5-diethylpyrrol, pyrrole, 5-phenyldipyrromethane and thiophene; these reactions were performed in DCM (Tab 3.1).

A direct coupling with the heterocycles mentioned above was again not successful, neither with the diol 18 nor its chloride analog 22. Therefore, a variety of different reactants was tested. The reaction conditions used for the different unsuccessful coupling reactions with 3,5-diethylpyrrole, pyrrole, 5-phenyl-dipyrromethane and thiophene are given in table 3.1. Since $\mathbf{1 8}$ appeared to be the most suitable compound, it was used in most of the reactions.

Table 3.1: Different reaction conditions used for the coupling of the pyrazole building blocks 18, 20, 21 and 22 with the different heterocycles at ambient temperature.

\begin{tabular}{l|c|c|c|c}
\hline Compound & Reactant & Equivalents & Solvent & Time \\
\hline $\mathbf{1 8 , 2 0 , 2 1 , 2 2} \mathbf{2 2}^{\mathrm{a}, \mathrm{b}, \mathrm{c}, \mathrm{d}}$ & - & - & $\mathrm{CH}_{2} \mathrm{Cl}_{2}$ & $2 \mathrm{~h} / 16 \mathrm{~h}$ \\
$\mathbf{1 8 , 2 0} \mathbf{2}^{\mathrm{a}, \mathrm{b}, \mathrm{c}, \mathrm{d}}$ & $\mathrm{BF}_{3} \cdot \mathrm{Et}_{2} \mathrm{O}$ & 2.9 & $\mathrm{MeCN}^{2}$ & $2 \mathrm{~h} / 16 \mathrm{~h}$ \\
$\mathbf{1 8}^{\mathrm{a}, \mathrm{b}, \mathrm{c}, \mathrm{d}}$ & $\mathrm{TFA}$ & $0.2 / 2.0$ & $\mathrm{CH}_{2} \mathrm{Cl}_{2}$ & $2 \mathrm{~h} / 16 \mathrm{~h}$ \\
$\mathbf{1 8}^{\mathrm{a}, \mathrm{d}}$ & $\mathrm{Amb}^{\mathrm{a}} 15$ & $0.2 / 2.0$ & $\mathrm{CH}_{2} \mathrm{Cl}_{2}$ & $2 \mathrm{~h} / 16 \mathrm{~h}$ \\
$\mathbf{1 8}^{\mathrm{a}, \mathrm{c}}$ & $\mathrm{KHSO}_{4} \cdot \mathrm{SiO}_{2}$ & $0.2 / 2.0$ & $\mathrm{CH}_{2} \mathrm{Cl}_{2}$ & $16 \mathrm{~h}$ \\
$\mathbf{1 8}^{\mathrm{c}}$ & $\mathrm{AcOH}$ & $0.2 / 2.0$ & $\mathrm{CH}_{2} \mathrm{Cl}_{2}$ & $16 \mathrm{~h}$ \\
$\mathbf{1 8}^{\mathrm{a}, \mathrm{c}}$ & $\mathrm{TsOH}$ & $0.2 / 2.0$ & $\mathrm{CH}_{2} \mathrm{Cl}_{2}$ & $16 \mathrm{~h}$ \\
\hline
\end{tabular}

[a] 3,5-diethylpyrrole. [b] pyrrole. [c] 5-phenyl-dipyrromethane. [d] thiophene.

The expected similar reactivity of the phenylacetylene pyrazole building blocks $\mathbf{1 8}$ and $\mathbf{2 2}$ in comparison to the previously used pyrazole building block with three phenyl groups in the backbone was not observed. This finding could not be explained so far, but other investigations have shown that the electronic structure of the pyrazole building block has a huge influence on its coupling reactions. Whereas 3,5-bis(chloromethyl)$1 H$-pyrazole does react with 3,5 -diethylpyrrole straight forward ${ }^{[46]}$ the corresponding 3,5-bis(chloromethyl)- $1 H$-triazole did not react at all. ${ }^{\text {95] }}$ 


\section{New Siamese-Twin Porphyrins}

The synthesis of new Siamese-twin porphyrins is of high interest, due to their interresting electronic structure, especially in metal complexes. Besides the planarization by removing steric pressure in the STPs backbone (Chapter 3), the variation of the aldehyde used for the cyclization reaction is another possibilty to change the molecule's physical properties.

A variation of all or some of the meso-groups perceivably modulates the electronic properties of the STP-macrocycle, even though the meso-phenyl groups are in the solid state idealized orthogonal to the mean plane of the position of the macrocycle they are attached to in the solid state. ${ }^{[10]}$ However, parallel to the findings for meso-arylporphyrins, some rotational freedom of the meso-phenyl groups can be assumed, with the transition state of the rotation possibly being stabilized by resonance effects. ${ }^{[96-98]}$ Even though the electronic effects, due to inductive effects of the meso-phenyl substituents, of varying meso-aryls are small, they are not negligible. ${ }^{[32,99-102]}$ meso-Alkylporphyrins are also electronically much distinct from their meso-aryl congeners. ${ }^{103]}$

With the use of electron withdrawing or donating substituents the oxidation or reduction of the corresponding metal complexes should be easier or harder to achieve. Furthermore it has been shown in literature, that slight changes in the porphyrin backbone by electron donating or withdrawing groups have an influence on the binding of substrates, for example oxygen to a cobalt TPP complex. ${ }^{[104]}$ The tuning of the redox potential can therefore help to find suitable metal complexes of the Siamese-twin porphyrin for the activation of small molecules.

\subsection{Synthesis of $\mathrm{X}_{\mathrm{LH}_{4}}$}

Of all the substituents on the Siamese-twin porphyrin framework, in principle, none are as readily varied as the meso-phenyl group located between two pyrrolic moieties (Fig. 4.1). ${ }^{[10,86]}$ All it requires is the variation of the arylaldehyde in the $3+3$ condensation step of pyrrole/pyrazole building block $\mathbf{1 3}$ with an arylaldehyde to produce porphyrinogen $\mathbf{X}_{\mathbf{L H}_{\mathbf{6}}}$, where $\mathbf{X}$ reflects the substitution pattern at the used arylaldehyde. This intermediate is subsequently oxidized to the final product ${ }^{\mathbf{X}} \mathbf{L H}_{\mathbf{4}}$ (Scheme 4.2). Its practical realization, however, proved to be more problematic. 
A series of arylaldehydes containing electron donating and withdrawing substituents as well as heptanal were used for the cyclization of $\mathbf{1 3}$ following the standard literature procedure for the preparation of $\mathbf{L H}_{\mathbf{4}} \cdot{ }^{[10,52]}$ All of the aldehydes tested resulted in the formation of the porphyrinogen $\mathbf{X}_{\mathbf{L}} \mathbf{H}_{\mathbf{6}}$ (Tab. 4.1).

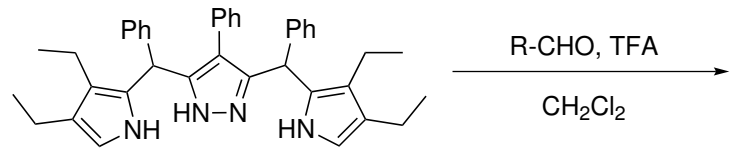

13

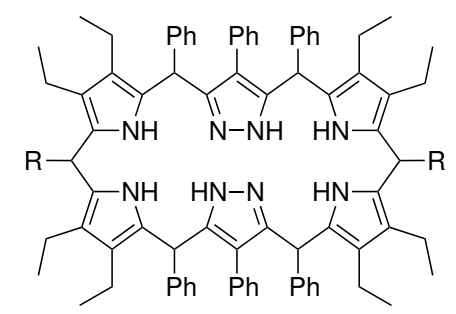

$\mathrm{X}_{\mathbf{L H}_{6}}$

Scheme 4.1: General synthesis of the Siamese-twin porphyrinogen ${ }^{\mathbf{L}_{\mathbf{6}}} \mathbf{\text { . }}$

The characterization of the porphyrinogen, as a mixture of multiple stereoisomers, is not trivial, because it is hard to detect in mass spectrometry and the proton NMR spectra are always very complicated due to the high number of stereoisomeres and therefore barely meaningful. ${ }^{[10]}$ Furthermore, the yields for the oxidation reaction with DDQ have been much higher, if the porphyrinogen was oxidized as soon as possible. Therefore the reaction mixture of the cyclization was always concentrated and filtered over a plug of basic aluminum oxide, where ${ }^{\mathbf{X}_{\mathbf{L}}} \mathbf{H}_{\mathbf{6}}$ was the only fraction passing. The porphyrinogen could be isolated and directly oxidized with DDQ to the corresponding expanded porphyrin $\mathbf{X}_{\mathbf{L H}}$ (Scheme. 4.2).

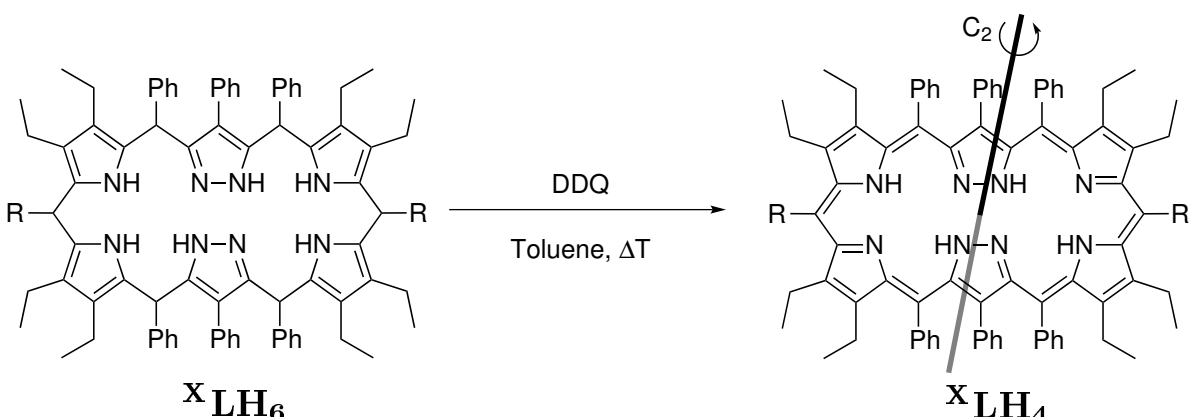

Scheme 4.2: Synthesis of the Siamese-twin porphyrins ${ }^{\mathrm{X}} \mathbf{L H}_{\mathbf{4}}$ with displayed $\mathrm{C}_{2}$ symmetry axis.

The new Siamese-twin porphyrins were sythesized according to literature, ${ }^{[10,86]}$ but the reaction time was lowered to 8 minutes and then the reaction mixture was rapidly cooled down to avoid overoxidation. ${ }^{[52]}$ The solvent was removed under reduced pressure and the resulting residue was suspended in a mixture of MTBE/ $\mathrm{CH}_{2} \mathrm{Cl}_{2} /$ EtOAC 10:3:1, filtered over a plug of basic aluminum oxide, where the expanded porphyrin ${ }^{\mathbf{X}} \mathbf{L} \mathbf{H}_{\mathbf{4}}$ was the only fraction passing, and dried under reduced pressure. ${ }^{[52]}$ The raw product was then further purified by column chromatography on silica with methanol as eluent. The isolated yields of the STP-derivatives $\mathbf{X}_{\mathbf{L H}}$ are listed in Table 4.1. 
Characterization of the resulting STPs was notably easier than the characterization of the porphyrinogens. The Siamese-twin porphyrin derivatives formed were spectroscopically characterized and showed all the expected analytical data. On a routine basis, the singly and doubly protonated $[\mathrm{M}+\mathrm{H}]^{+}$and $[\mathrm{M}+2 \mathrm{H}]^{2+}$ species could be observed in the $\mathrm{ESI}^{+}$ mass spectra, another indication for the relative pronounced electronic independence of each pyrrolic binding pocket in the STPs, shown previously also in solution state investigations. ${ }^{[10]}$

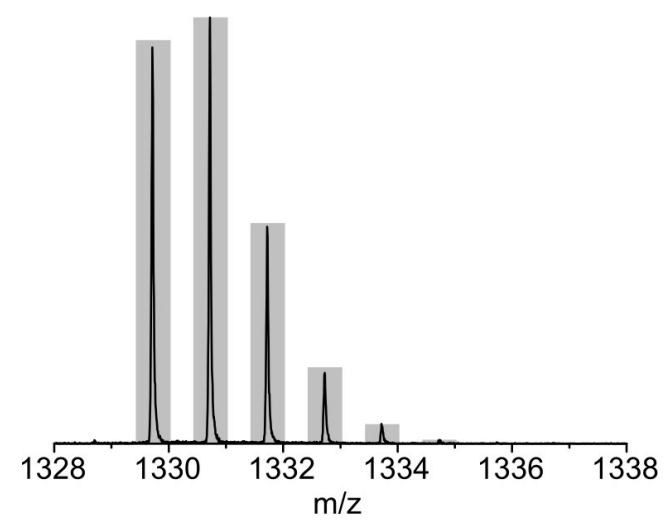

(a)

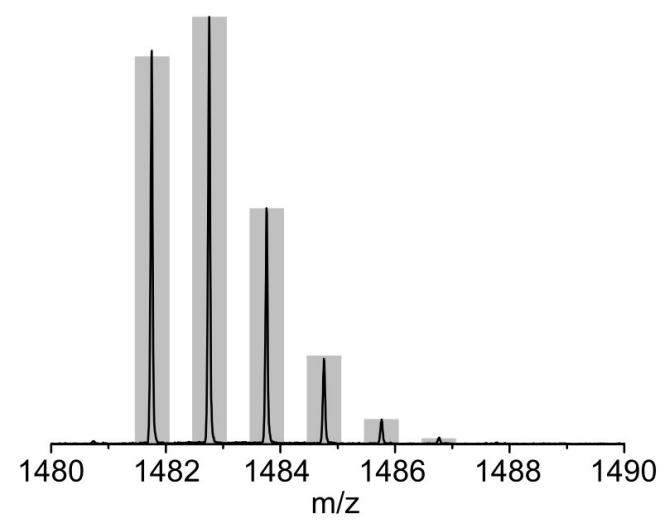

(c)

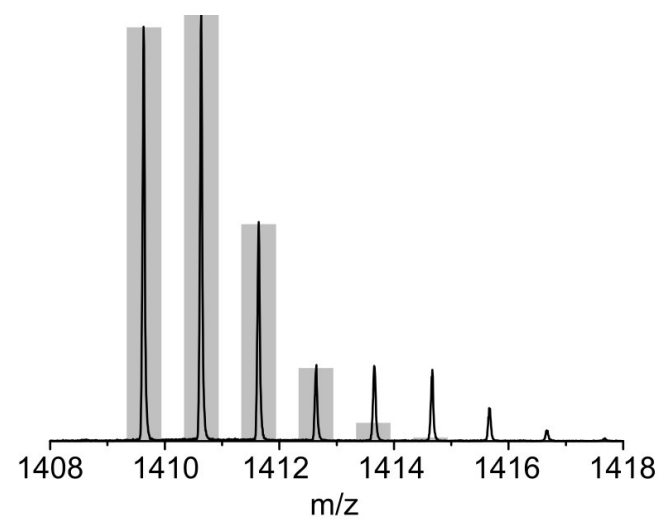

(e)

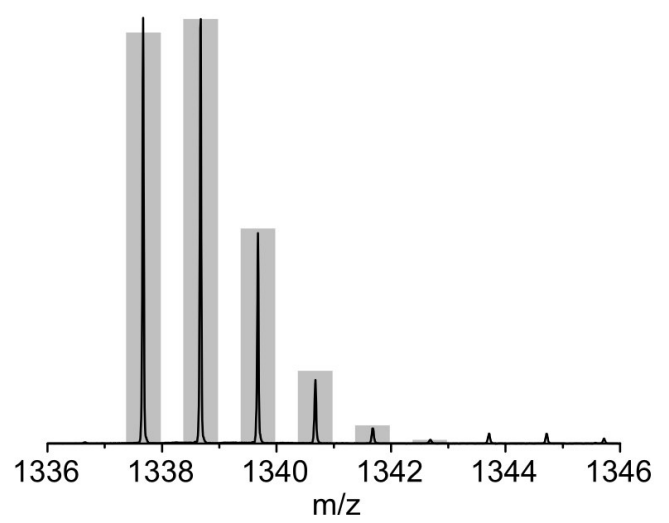

(b)

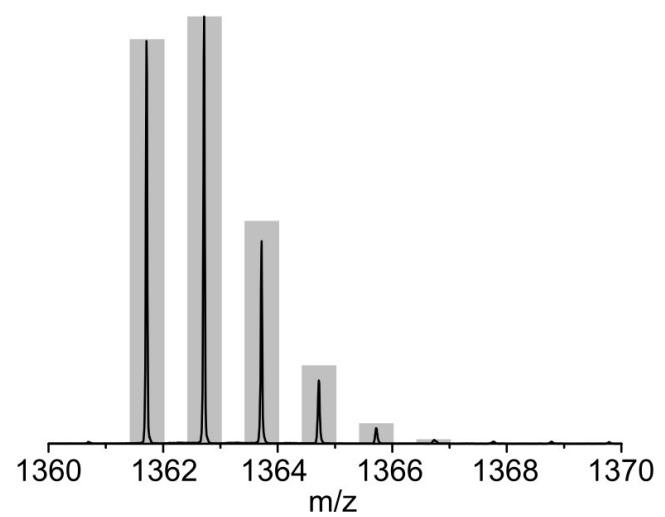

(d)

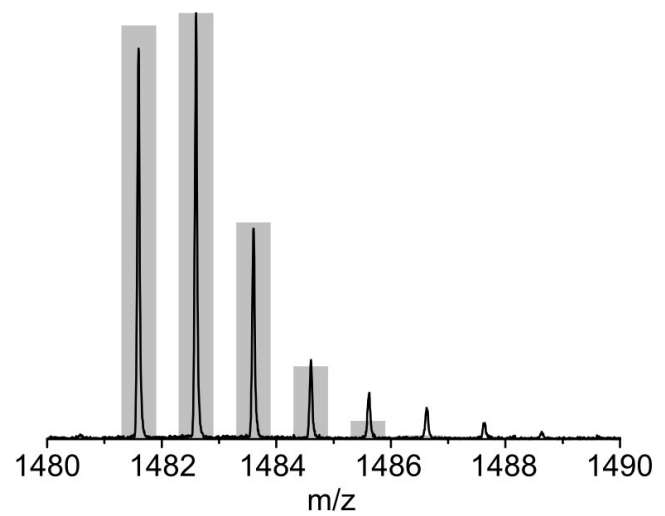

(f)

Figure 4.1: Cut-out HRMS $\mathrm{ESI}^{+}$spectra of the species $[\mathrm{M}+\mathrm{H}]^{+}$(black) of ${ }^{p \mathrm{Me}} \mathbf{L H}_{4}$ (a), ${ }^{p \mathrm{~F}} \mathrm{LH}_{4}$ (b), ${ }^{\mathrm{MeO}} \mathrm{LH}_{4}$ (c), ${ }^{p \mathrm{MeO}} \mathrm{LH}_{4}$ (d), ${ }^{\text {tri-F }} \mathrm{LH}_{4}$ (e) and penta-F $\mathrm{LH}_{4}$ (f) as well as the calculated isotopic pattern (grey bars). 
Table 4.1: Experimental findings and Hammett parameters of the aldehydes used.

\begin{tabular}{|c|c|c|c|c|}
\hline Aldehyde & Label & Yield / \%[a] & $\sum \sigma^{[\mathrm{b}]}$ & $\lambda_{\max } / \mathrm{nm}^{[\mathrm{c}]}$ \\
\hline $\mathrm{PhCHO}$ & $\mathrm{LH}_{4}$ & $50^{[10]}(23)^{[\mathrm{d}]}$ & 0 (by definition) & 640 \\
\hline 4- $\mathrm{CH}_{3}-\mathrm{PhCHO}$ & ${ }^{p \mathrm{Me}} \mathrm{LH}_{4}$ & 24 & -0.17 & 640 \\
\hline $4-\mathrm{F}-\mathrm{PhCHO}$ & ${ }^{p \mathrm{~F}} \mathrm{LH}_{4}$ & 9 & 0.06 & 637 \\
\hline $3,4,5-\mathrm{OCH}_{3}-\mathrm{PhCHO}$ & ${ }^{\mathrm{MeO}_{2} \mathrm{LH}_{4}}$ & 15 & -0.03 & 640 \\
\hline $4-\mathrm{OCH}_{3}-\mathrm{PhCHO}$ & ${ }^{p \mathrm{MeO}} \mathrm{LH}_{4}$ & 27 & -0.27 & 640 \\
\hline 2,4,6-F-PhCHO & ${ }^{t r i-}-{ }^{-} \mathbf{L H}_{4}$ & -[e] & 0.06 & - \\
\hline $2,3,4,5,6-\mathrm{F}-\mathrm{PhCHO}$ & penta- ${ }^{-} \mathbf{L H}_{4}$ & _[e] & 0.74 & - \\
\hline $\mathrm{n}-\mathrm{C}_{6} \mathrm{H}_{13} \mathrm{CHO}$ & - & n.d. ${ }^{[\mathrm{f}]}$ & - & - \\
\hline
\end{tabular}

[a] Isolated yield of (micro-)crystalline material based on pyrrole/pyrazole building block $\mathbf{1 3}$ used in synthesis. [b] Sum of Hammett parameters of a single meso-aryl group. ${ }^{[105]}$ [c] Longest wavelength of absorption (in $\mathrm{CH}_{2} \mathrm{Cl}_{2}$ ) cf. also Figure 4.3. [d] The high yield reported by FRENSCH ${ }^{[10]}$ could not be reproduced on a routine basis at larger scales reported here. [e] Detected by MS. No material isolated. [f] Not detected.

Remarkable are the diverse isolated yields of the products, varying from traces for penta-F $\mathbf{L H}_{\mathbf{4}}$, to $9 \%$ for ${ }^{\boldsymbol{p} \mathbf{F}} \mathbf{L} \mathbf{H}_{\mathbf{4}}$, to satisfying yields of $24 \%$ and $27 \%$ for ${ }^{\boldsymbol{p} \mathbf{M e}} \mathbf{L} \mathbf{H}_{\mathbf{4}}$ and ${ }^{p \mathrm{MeO}} \mathbf{L H}_{4}$, respectively. While aldehydes carrying the strongly electron-withdrawing substituents, such as 2,4,6-trifluoro- and 2,3,4,5,6-pentafluorobenzaldehyde, were expected to be more susceptible to nucleophilic attack and thus to react faster than the electron-rich aldehydes, this did not translate into higher isolated yields of the final product. This effect may be due to a decreased reactivity of the porphyrinogen precursor in the oxidation step. Thus it was found that the use of meso- $\mathrm{C}_{6} \mathrm{~F}_{5}$ groups did not have any benefits, even though this meso-substituent has been particularly popular in the field of expanded porphyrinic macrocycles. ${ }^{[26,106-113]}$ Inversely, the somewhat more electron-rich aldehydes 4-methyl- and 4-methoxybenzaldeyde produced high yields of product. Heptanal led to the formation of an unstable product that could not be characterized as an STP by HR-MS and UV-vis spectroscopy. Thus, $p$-tolylaldehyde, 4-fluorobenzaldehyde, 4-methoxybenzaldehyde and 3,4,5-trimethoxybenzaldehyde allowed the synthesis of the corresponding STPs in multi-100 mg batches using 2 gram of 13 in $350 \mathrm{~mL}$ solvent. The degree of crystallinity of the substituted STPs also differs significantly from each other, contributing to the higher yields for some of the products.

The proton NMR spectrum of each STP was recorded at $-35{ }^{\circ} \mathrm{C}$ due to signal broadening at higher temperatures (Fig. 4.2). As can be seen in the inset the introduction of three methoxy groups resulted in a more unstable expanded porphyrin which always showed decomposition in solution, resulting in more than three methoxy peaks. This effect was much weaker, yet still observable when 4-methoxybenzaldehyde was used. Like $\mathbf{L H}_{4}$ all new STPs are $\mathrm{C}_{2}$ symmetric (Fig. 4.2), resulting in half a set of proton and carbon NMR signals. ${ }^{[10]}$ 


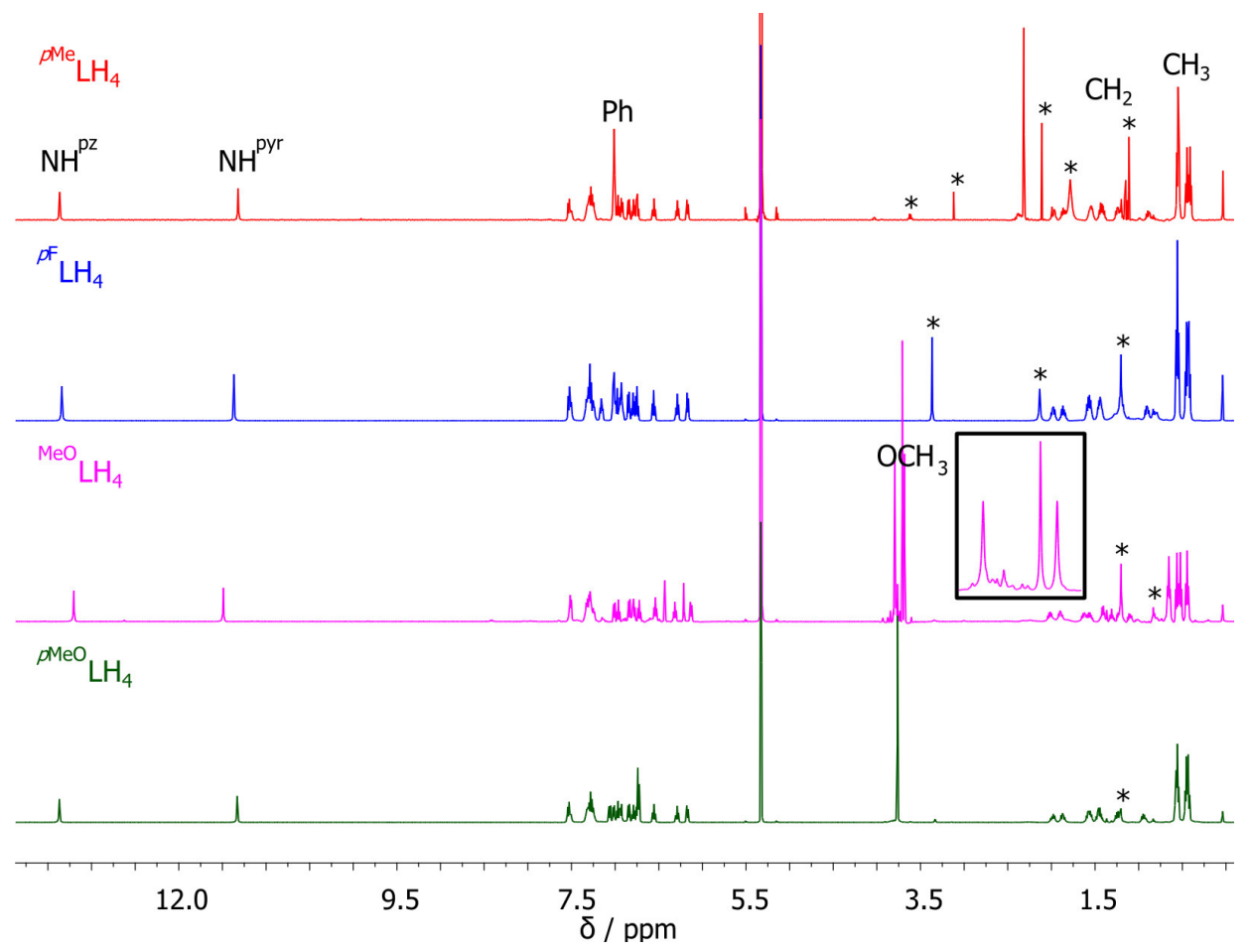

Figure 4.2: ${ }^{1} \mathrm{H}$ NMR spectra of the different STPs in $\mathrm{CD}_{2} \mathrm{Cl}_{2}$ at $238 \mathrm{~K}$. The inset shows the region of the methoxy groups of the corresponding spectrum. Solvent impurities are marked with an asterisk.

The UV-vis spectra of the STPS $\mathbf{X}_{\mathbf{L H}}$ are all as expected, and nearly identical to the all-phenyl derivative $\mathbf{L H}_{\mathbf{4}}$, because the phenyl group's inductive effect does not affect the HOMO-LUMO gap. The differences observed in their extinction coefficients are within the error of the measurement (Fig. 4.3).

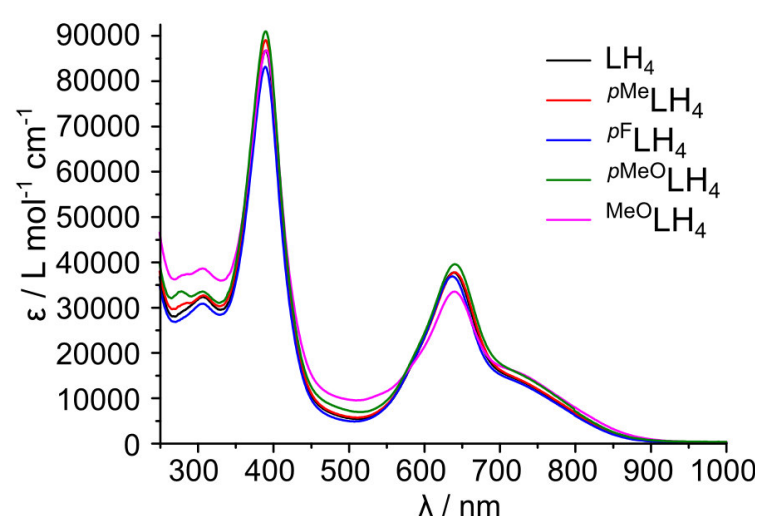

Figure 4.3: UV-vis spectra of the different STPs in $\mathrm{CH}_{2} \mathrm{Cl}_{2}$ at ambient temperature.

However, the substituents modulate to a noticeable degree the solubility of the STPs. In particular, the presence of methoxy groups remarkably increased the solubility of the SPTs in polar solvents like acetone or methanol, complicating the cleanup and crystallization. Therefore no crystal structures of the Siamese-twin porphyrins ${ }^{p \mathbf{M e O}} \mathbf{L H}_{\mathbf{4}}$ and 
${ }^{\mathrm{MeO}} \mathbf{L H}_{4}$ could be obtained so far. Nevertheless, the single crystal X-ray structures of ${ }^{p \mathrm{~F}} \mathbf{L H}_{4}$ (Fig. 4.4) and ${ }^{p \mathrm{Me}} \mathbf{L H}_{\mathbf{4}}$ (not shown, see Appendix) could be determined.

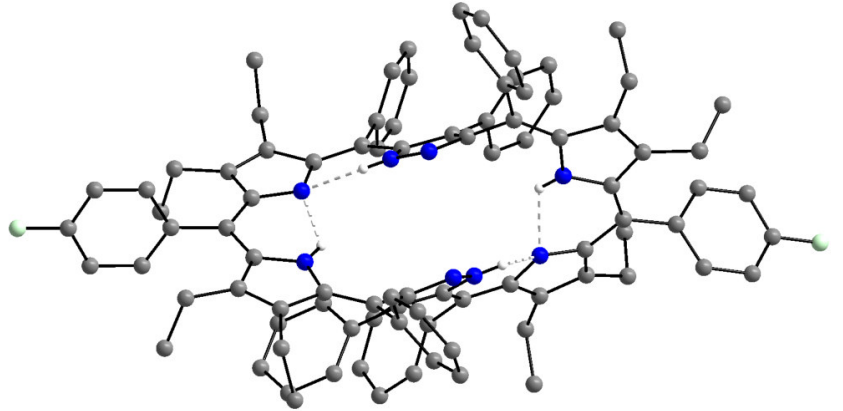

(a)

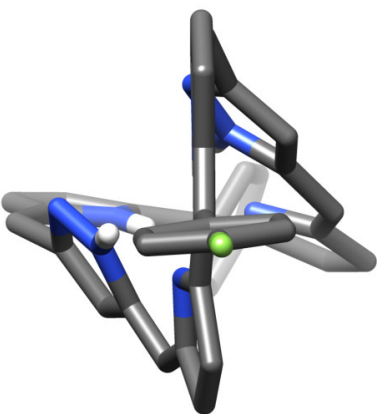

(b)

Figure 4.4: Ball-and-stick model of the molecular structure of ${ }^{p \mathrm{~F}} \mathbf{L H}_{4}$ (a) top view and (b) side view as stick model along the pyrrole/pyrrole meso-position axis. All carbonbound hydrogen atoms, disorder and solvent molecules were omitted for clarity (grey: carbon, blue: nitrogen, green: flourine, white: hydrogen).

The framework structures of $\mathbf{L H}_{\mathbf{4}},{ }^{p \text { Me }} \mathbf{L H}_{4}$, and ${ }^{p \mathbf{F}} \mathbf{L H}_{\mathbf{4}}$ are all near-identical to each other, albeit the compounds crystallized in different space groups $\left(\mathbf{L H}_{4}: \mathrm{P} \overline{1},{ }^{p \mathbf{M e}} \mathbf{L H}_{4}\right.$ and $\left.{ }^{p \mathbf{F}} \mathbf{L H}_{\mathbf{4}}: \mathrm{P} 2_{1} / \mathrm{c}\right)$. Thus, the change of the two meso-substituents did not alter the twisted conformation of the macrocycle (Fig. 4.5). This underlines the conformational rigidity of the macrocycle imposed by the substituents, shown previously. ${ }^{[53-55]}$
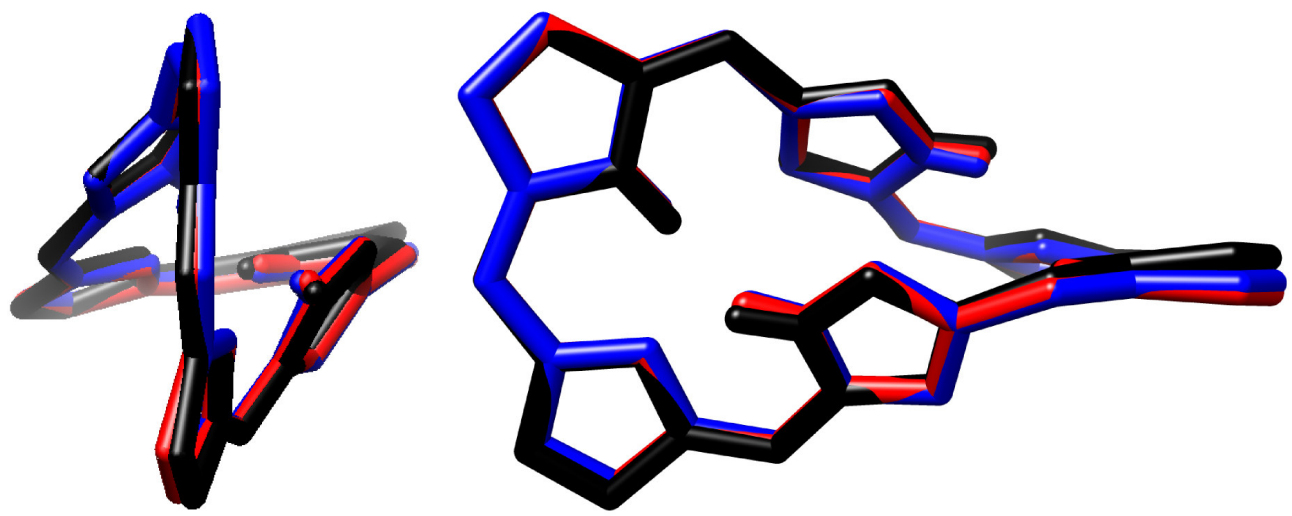

Figure 4.5: Overlay of the macrocycle core structures of $\mathbf{L H}_{\mathbf{4}}{ }^{[52]}$ (black), ${ }^{\boldsymbol{p} \mathbf{M e}} \mathbf{L H}_{\mathbf{4}}$ (red) and ${ }^{p} \mathbf{F} \mathbf{L H}_{4}$ (blue) as determined by single crystal X-ray crystal diffractometry indicating near-identical conformations. For details see appendix.

Due to the enhanced solubility of ${ }^{p \mathrm{MeO}} \mathbf{L H}_{4}$ and ${ }^{\mathrm{MeO}} \mathbf{L H}_{4}$ no solid state crystal structure could be obtained so far. The molecular structure of these derivatives was calculated by DFT methods using the ORCA program, because DFT calculations have previously been shown to predict the conformations of STPs and its metal complexes with high fidelity. ${ }^{[10,53-55]}$ The computed conformation of ${ }^{p \mathrm{MeO}} \mathbf{L H}_{\mathbf{4}}$ and ${ }^{\mathrm{MeO}} \mathbf{L H}_{\mathbf{4}}$ indicated the retention of the conformation observed and calculated for $\mathbf{L H}_{\mathbf{4}}$. The perpendicular arrangement for the aryl groups prevents any steric clashes between the aryl substituents and the neighboring $\beta$-ethyl groups (Fig. 4.6). 


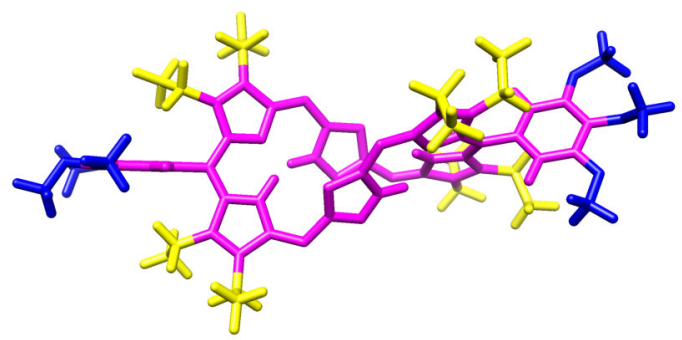

(a)

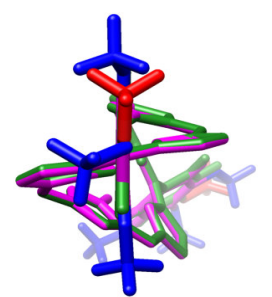

(b)

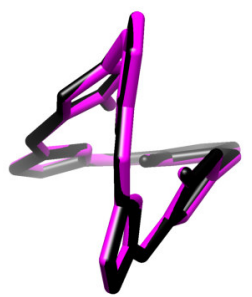

(c)

Figure 4.6: (a) Calculated core structure of ${ }^{\mathrm{MeO}} \mathbf{L H}_{4}$ without the phenyl groups of the pyrrolpyrazol building block (yellow: Et, blue: $\mathrm{MeO}$ ), (b) side view of the overlay of ${ }^{\mathrm{MeO}} \mathbf{L H}_{4}$ (magenta with blue methoxy groups) and ${ }^{p \mathrm{MeO}} \mathrm{LH}_{4}$ (green with red methoxy groups) and (c) side view of the overlay of $\mathbf{L H}_{4}$ (black) and ${ }^{\mathrm{MeO}} \mathbf{L H}_{4}$ (magenta).

The methoxy groups are almost in a parallel arrangement with the phenyl ring (Fig. 4.6 (b)) and therefore far enough away from the $\beta$-ethyl groups. Thus, the methoxy groups have no influence on the twist of the Siamese-twin porphyrin (Fig. 4.6 (c)).

\subsection{Copper Complexes of ${ }^{\mathrm{LH}_{4}}$}

The STP dicopper complex $\mathbf{L} \mathbf{C u}_{\mathbf{2}}$ exhibited well-defined redox peaks in its cyclic voltammogram, associated with oxidations that mainly take place at the dipyrromethene subunits of the macrocycle (cf. Fig. 1.8). ${ }^{[53]}$ It thus could be expected that the oxidation

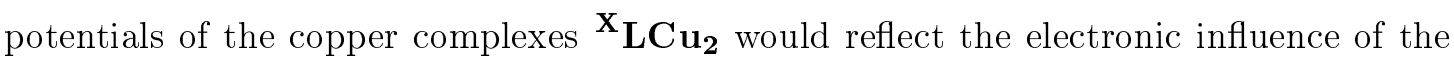
meso-aryl groups. Electron withdrawing groups in the phenyl's backbone should hamper the oxidation, resulting in a shift to higher electric potential, whereas the opposite should be true for electron donating groups. This is indeed the case (Fig. 4.8)

The blue dicopper complexes ${ }^{p \mathrm{Me}} \mathbf{L} \mathbf{C u}_{2},{ }^{p \mathrm{~F}} \mathbf{L} \mathbf{C u}_{2},{ }^{\mathrm{MeO}} \mathbf{L} \mathbf{C u}_{2}$ and ${ }^{p \mathbf{M e O}} \mathbf{L C u}_{2}$ have been synthesized by reaction of the free base STP with a source of copper(II) in a polar solvent (Scheme 4.3), as described previously for the formation of $\mathbf{L} \mathbf{C u}_{\mathbf{2}}{ }^{[53,54]} \mathrm{but}$ using a modified purification protocol.
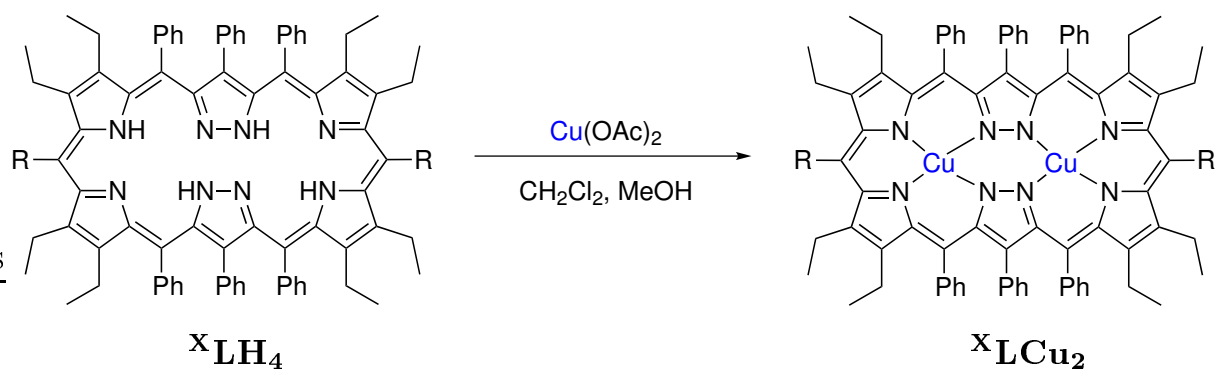

Scheme 4.3: Synthesis of the copper complexes ${ }^{\mathbf{x}} \mathbf{L C u}_{2}$ of the STP according to literature. ${ }^{[53,54,86]}$ 
The solvent of the reaction mixture was removed under reduced pressure and the residue was dissolved in dichloromethane and filtered over a plug of basic aluminium oxide. The only fraction passing was the desired blue copper complex. Again the isolated yields differ widely, varying from low yields of $18 \%$ for ${ }^{p \mathbf{F}} \mathbf{L} \mathbf{C u}_{2}$, to satisfying yields of $52 \%$, $60 \%$ and $67 \%$ for ${ }^{p \mathbf{M e O}_{\mathbf{L C u}}}{ }^{p}{ }^{p \mathbf{M e}} \mathbf{L} \mathbf{C u}_{2}$ and ${ }^{\mathrm{MeO}} \mathbf{L} \mathbf{C u}$, respectively.

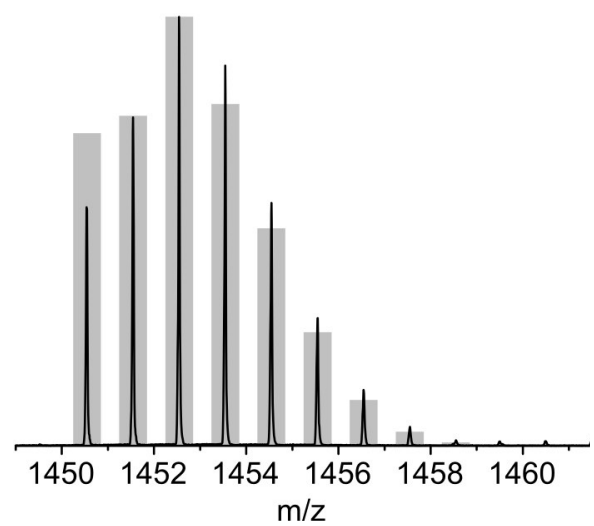

(a)

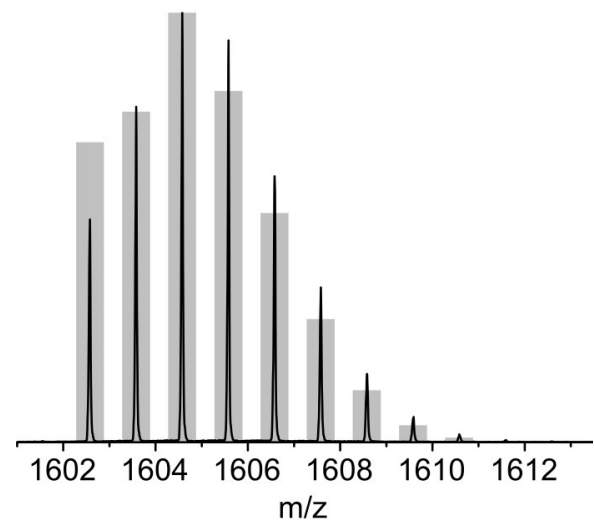

(c)

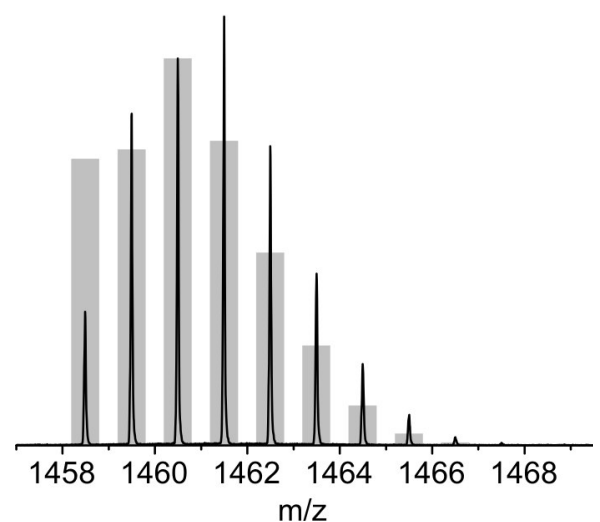

(b)

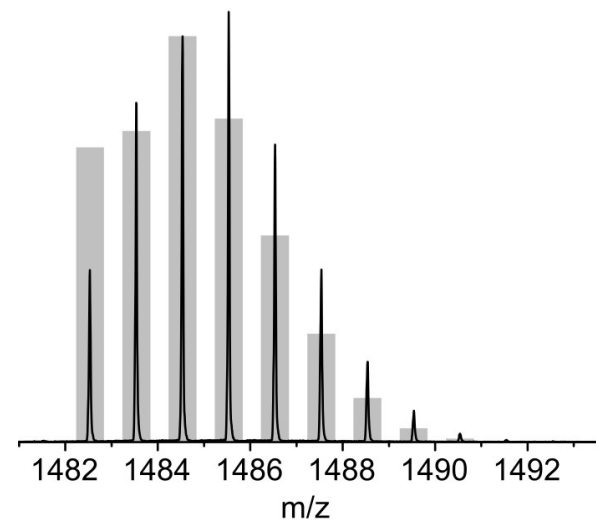

(d)

Figure 4.6: Cut-out HRMS ESI ${ }^{+}$spectra of the species $[\mathrm{M}]^{+}$(black) of ${ }^{p \mathrm{Me}}{ }^{\mathbf{L}} \mathbf{C u}_{2}(\mathbf{a}),{ }^{p \mathrm{~F}} \mathbf{L} \mathbf{C u} \mathbf{u}_{2}$ (b), ${ }^{\mathrm{MeO}} \mathbf{L C u}_{2}$ (c) and ${ }^{p \mathrm{MeO}} \mathbf{L C u}_{2}$ (d) and the calculated isotopic pattern (grey bars).

On a routine basis, the singly oxidized $[\mathrm{M}]^{+}$species are observed in the $\mathrm{ESI}^{+}$mass spectra. The isotopic pattern does not always fit because of an overlap of the singly oxidized species $[\mathrm{M}]^{+}$and the minor species $[\mathrm{M}+\mathrm{H}]^{+}$, one mass unit heavier, resulting in a too high third and too low first peak of the isotopic pattern. This difference is highest for ${ }^{p \mathrm{~F}} \mathbf{L} \mathbf{C u}_{\mathbf{2}}$ (Fig. $4.6(\mathbf{b})$ ).

Mirroring the trends seen for the free base expanded porphyrins, the UV-vis spectra of the dicopper complexes were also near-identical (Fig. 4.7). The absorption band marked with an asterisk (Fig. 4.7) most likely results from a slight oxidation of the corresponding copper complex. A similar absorption band has been observed in the oxidizied copper complex $\mathbf{L C u}_{2}+.{ }^{[53]}$ 


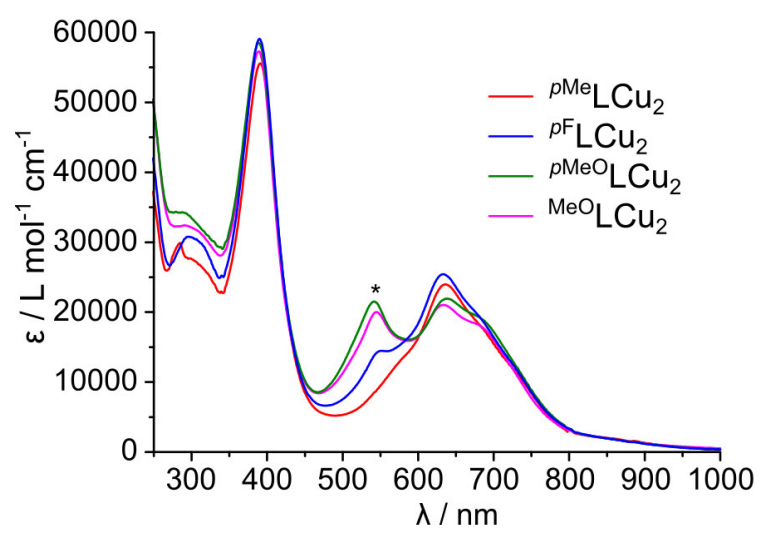

Figure 4.7: UV-vis spectra of the different copper complexes ${ }^{\mathbf{x}_{\mathbf{L}}} \mathbf{L C u}_{2}\left({ }^{*}\right.$ likely some oxidation product) in $\mathrm{CH}_{2} \mathrm{Cl}_{2}$ at ambient temperature.

Cyclic and square wave voltammetry measurements of the dicopper complexes ${ }^{\mathbf{L}} \mathbf{C u}_{\mathbf{2}}$ (Fig. 4.8) showed the expected two oxidation events in the potential range from -0.8 to $+0.4 \mathrm{~V}$ vs. $\mathrm{Fc} / \mathrm{Fc}^{+} .{ }^{[53,55]}$ The potentials are shifted to higher values with the electron withdrawing fluorine substituent and are shifted to lower values with electron-donating substituents, with the shifts conforming to a linear Hammett plot (Fig. 4.9). Only the first oxidation peak of the trimethoxyphenyl-substituted complex ${ }^{\mathrm{MeO}}{ }_{\mathbf{L C u}} \mathbf{C u}_{2}$ lies outside this trend.

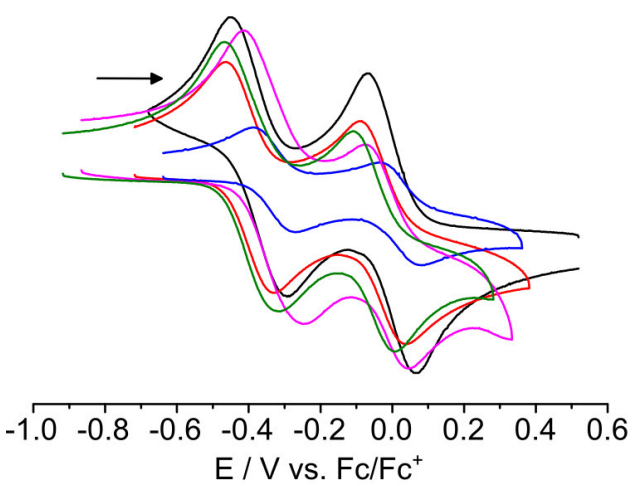

(a)

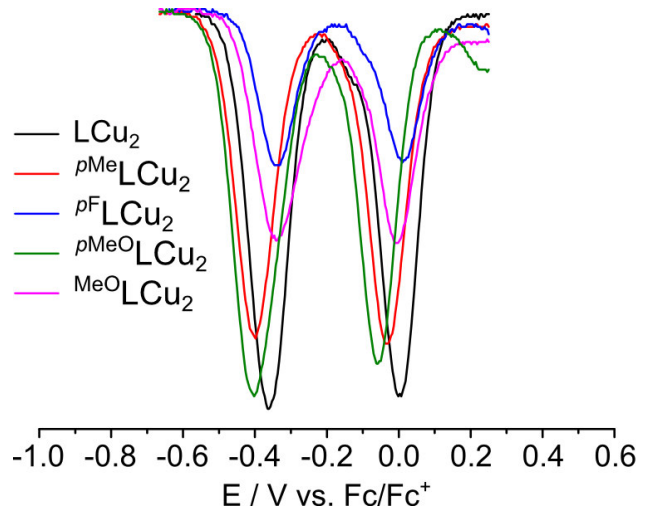

(b)

Figure 4.8: (a) Cyclic and (b) square wave voltammogram of the new copper complexes in comparison to $\mathbf{L C u}_{2}\left(\mathrm{CH}_{2} \mathrm{Cl}_{2}, 0.1 \mathrm{M}\left[\mathrm{Bu}_{4} \mathrm{~N}\right] \mathrm{PF}_{6}\right)$ at a scan rate of $100 \mathrm{mV}$. ${ }^{[53]}$

One explanation of the deviation of ${ }^{\mathrm{MeO}} \mathbf{L} \mathbf{C u}_{2}$ could be its instabillity in solution. Decomposition during the measurement is supported by the fact that the longer the measurement was running the more small additional peaks arose in case of the two methoxy

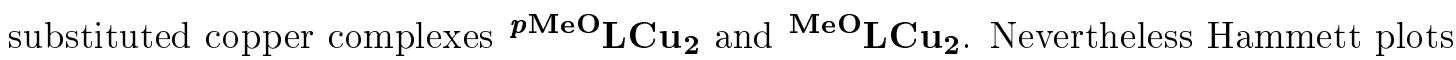
of the two oxidations of the copper complexes clearly show a linear correlation between the electronic structure at the dipyrromethene subunits of the Siamese-twin porphyrin and the substitution of the phenyl group (Fig. 4.9). 


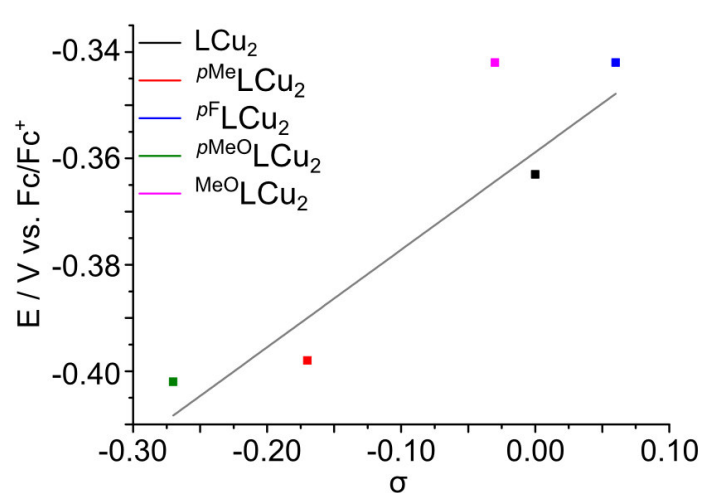

(a)

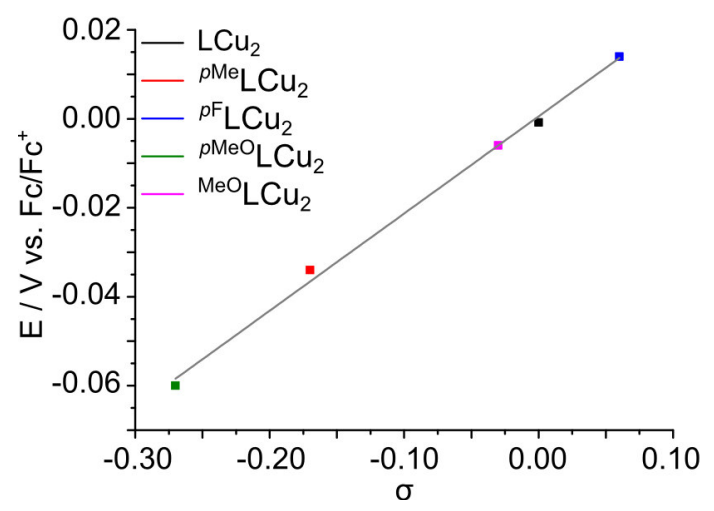

(b)

Figure 4.9: Hammett plot of the first (a) and second (b) oxidation of the copper complexes with linear regressions (grey). The data point in (a) corresponding to ${ }^{\mathrm{MeO}} \mathrm{LCu}_{2}$ is excluded from the linear regression, because it lies outside the trend in SWV (cf. Fig. 4.8).

Interestingly, the exchange of one or both copper ions with one or two nickel ions has a much bigger influence on the corresponding redox potentials than the substitution patterns of the two meso-aryl groups (Table 4.2). In comparison, the shifts observed in the corresponding meso-tetraarylporphyrin copper complexes $\mathrm{T}(p \mathrm{X}) \mathrm{PPCu}$ upon variation of the (four) meso-aryl groups are significantly larger while the shifts when switching the metal ion from copper to nickel are much smaller than those observed for the STP complexes. ${ }^{[56-58,101,114,115]}$ This further underlines the principally different electronic structures of the non-macrocycle-aromatic STPs compared to the aromatic porphyrins.

Table 4.2: Potential difference of the first two oxidations of the different metal complexes in comparison to $\mathbf{L C u}_{2}{ }^{[\mathrm{a}]}$ and the corresponding meso-tetrakis(aryl)porphyrin complexes.

\begin{tabular}{|c|c|c|c|}
\hline Complex & $\Delta E \mathrm{Ox} 1 / \mathrm{mV}$ & $\Delta E \mathrm{Ox} 2 / \mathrm{mV}$ & Ref. \\
\hline $\mathrm{LCu}_{2}$ & $0^{[\mathrm{b}]}$ & $0^{[\mathrm{b}]}$ & [53] \\
\hline$p{ }^{\mathrm{Me}} \mathrm{LCu}_{2}$ & -35 & -34 & - \\
\hline$p \mathrm{~F}_{\mathbf{L C u}}$ & 20 & 14 & - \\
\hline${ }^{\mathrm{MeO}} \mathrm{LCu}_{2}$ & -40 & -60 & - \\
\hline$p \mathrm{MeO}_{\mathbf{L C u}}$ & 20 & -6 & - \\
\hline LCuNi & 30 & 260 & [55] \\
\hline $\mathbf{L N i}_{2}$ & 210 & 320 & [55] \\
\hline $\mathrm{TPPCu}^{[\mathrm{c}]}$ & $0^{[b]}$ & $0^{[\mathrm{b}]}$ & [56] \\
\hline $\mathbf{T}(p \mathbf{M e}) \mathbf{P P C u}{ }^{[\mathrm{d}]}$ & -70 & -20 & [101] \\
\hline $\mathbf{T}(p \mathrm{OMe}) \mathbf{P P C u}{ }^{[\mathrm{e}]}$ & -140 & - & [114] \\
\hline $\mathbf{T P P N i} \mathbf{i}^{[\mathrm{f}]}$ & 10 & 60 & [57] \\
\hline
\end{tabular}

[a] Values as determined by square wave voltammetry (in $\mathrm{CH}_{2} \mathrm{Cl}_{2}, 0.1 \mathrm{M}\left[\mathrm{Bu}_{4} \mathrm{~N}\right] \mathrm{PF}_{6}$ ) cf. to Fig. 4.8. [b] By definition. [c] [meso-tetraphenyl-porphyrinato]copper(II). [d] [meso-tetrakis(4-methylphenyl)porphyrinato]-copper(II). [e] [meso-tetrakis(4methoxyphenyl)porphyrinato]-copper(II). [f] [meso-tetraphenyl-porphyrinato]-nickel(II). 
Partial oxidation of the copper complexes ${ }^{\mathbf{X}} \mathbf{L} \mathbf{C u}_{\mathbf{2}}$ observed in UV-vis spectroscopy (Fig. 4.7) could be confirmed by thin layer chromatography, with the presence of two additional weak violet and pink spots, giving the same mass spectrum than the blue copper complexes. Former studies have shown that a color change to violet and purple came along with the oxidation of $\mathbf{L} \mathbf{C u}_{2} \cdot{ }^{[53,55]}$ However, EPR measurements of the different copper complexes (Fig. 4.10) showed no signal for the oxidized complexes when compared with the spectrum of $\mathbf{L} \mathbf{C u}_{2}{ }^{+}{ }^{[53]}$ It can therefore be assumed that the amount of oxidized ${ }^{\mathbf{X}} \mathbf{L} \mathbf{C u}_{\mathbf{2}}$ in the samples is rather small.

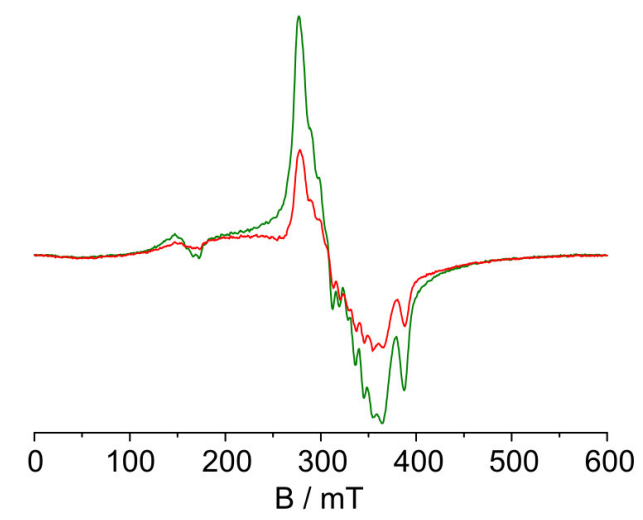

Figure 4.10: Selected EPR spectra of the copper complexes ${ }^{p \mathrm{Me}} \mathbf{L C} \mathbf{u}_{2}$ (red) and ${ }^{p \mathrm{MeO}} \mathbf{L C u}_{2}$ (green) in $\mathrm{CH}_{2} \mathrm{Cl}_{2}$ at $150 \mathrm{~K}$ being very similar.

Furthermore the EPR spectra of ${ }^{\mathbf{X}} \mathbf{L} \mathbf{C u}_{\mathbf{2}}$ were all very similar, as expected. Even though the elctronic structure of the dipyrromethane subunit of the expanded porphyrins ${ }^{\mathbf{L}_{\mathbf{L}}} \mathbf{H}_{\mathbf{4}}$ should be influenced by the different arylsubstituents (Fig. 4.8) this obviously does not effect the EPR spectra, due to still identical copper environment. 



\section{Iron Complexes of the Siamese-Twin Porphyrin}

In analogy to the copper and nickel complexes of the Siamese-twin porphyrin, ${ }^{[10,53-55]}$ diiron and monoiron complexes of the parent STP $\mathbf{L H}_{\mathbf{4}}$ have been synthesized and characterized in the oxidation states of + II and + III. First small molecule activation reactions have been done, providing further evidence for the different electronic structure of a regular porphyrin and the Siamese-twin porphyrin (Chap. 7).

Until now a selective (temperature dependent) monometalation of the regular Siamesetwin porphyrin $\mathbf{L H}_{\mathbf{4}}$ was only possible in the case of nickel. ${ }^{[55]}$ In the case of copper and iron no temperature dependend monometalation could be observed. Even if only one equivalent of metal salt was used a mixture of free base Siamese-twin porphyrin and the dimetal complex was formed. Prior to the diiron complex of the Siamese-twin porphyrin, a number of different monoiron complexes $\left(\mathbf{L N i F e C l}, \mathbf{L N i F e}, \mathbf{L H}_{2} \mathbf{F e C l}, \mathbf{L C u F e C l}\right)$ have been synthesized with the use of the mononickel complex $\mathbf{L H}_{\mathbf{2}} \mathbf{N i}$ and according to recent observations by VOGEL. ${ }^{[116]}$ A monoiron complex would clearly facilitate the characterization of the iron ion's electronic structure, since cooperativity effects in the diiron complex hamper a disctinct assignment. Especially in Mößbauer spectroscopy the presence of only one iron atom simplifies its characterization tremendously.

\subsection{Iron Nickel Complexes of the Siamese-Twin Porphyrin}

The complex LNiFe is most suitable for the convenient characterization of an iron ion's electronic structure due to the low spin nickel(II) spin state $(S=0)$ in a square planar complexation geometry. Therefore the nickel(II) is EPR silent and does not contribute to magnetic susceptibility measurements, making the determination of the iron ion's spin state straightforward. Furthermore the synthesis of $\mathbf{L H}_{\mathbf{2}} \mathbf{N i}$ is well established and the nickel ion's electronic structure is well understood. ${ }^{[54,55]}$

Interestingly the use of $\mathrm{Fe}(\mathrm{OAc})_{2}$ did not lead to the formation of any iron complex at all, unlike the acetate salts of nickel and copper, ${ }^{[54,55]}$ even if the amount of iron acetate was raised or the reaction time was extended from hours up to days. With the use of $\mathrm{Fe}\left(\mathrm{BF}_{4}\right)_{2}$ or $\mathrm{FeCl}_{2}$ in the presence of an external base heterobimetallic nickel iron(III) complexes could be synthesized under aerobic conditions according to HRMS 
measurements. Aerobic conditons were applied initially, because iron porphyrins have been shown to be most stable in their ferric oxidation state and they can be easily reduced to the corresponding iron(II) complex later on. ${ }^{[8,9]}$

Even though complexation reactions with $\mathrm{Fe}\left(\mathrm{BF}_{4}\right)_{2}$ led to the corresponding heterobimetallic nickel iron complex, the complex could not be isolated. The complex showed fast decomposition during liquid chromatography, and its solubility in organic solvents was enhanced in comparison to the previously synthesized nickel and copper complexes. ${ }^{[10,53,55,86]}$ The stability of the corresponding ferric complex $\mathbf{L N i F e C l}$ was higher, and its clean up was remarkably easier.

\subsubsection{Synthesis of LNiFeCl}

Complexation with $\mathrm{FeCl}_{2}$ under aerobic conditions, which results in an oxidation of the iron(II) ion subsequent to its complexation, was promising due to the iron ion's affinity to coordinate an axial ligand in the oxidation state of + III. Consequently, a clean conversion into the ferric complex $\mathbf{L N i F e C l}$ was expected. Indeed $\mathbf{L N i F e C l}$ could be synthesized with the use of iron(II) chloride and an external base. The complexation reaction with $\mathrm{FeCl}_{2}$ alone did not yield any product, like observed in porphyrin and/or corrole chemistry before. ${ }^{[8,117]}$ The metal insertion is assumed to be a concerted mechanism. ${ }^{[32,118]}$ The NH groups are only sligthly acidic and can most likely only be deprotonated with strong acids like trifluoroacetic acid, depending on the electronic structure of the porphyrin. ${ }^{[32]}$ During metalation, deprotonation of the NH groups only occurs when the metal ion/complex is already in close proximity. Conversely, incoorporation of a metal ion only occurs when a base is present. ${ }^{[118]}$ Therefore, deprotonation of the porphyrin's $\mathrm{NH}$ groups does not occure in the presence of $\mathrm{FeCl}_{2}$ only, because the basicity of chloride is not high enough. ${ }^{[32]}$ However, with the use of additional sodium acetate, the formation of $\mathbf{L N i F e C l}$ could be achieved (Fig. 5.1).

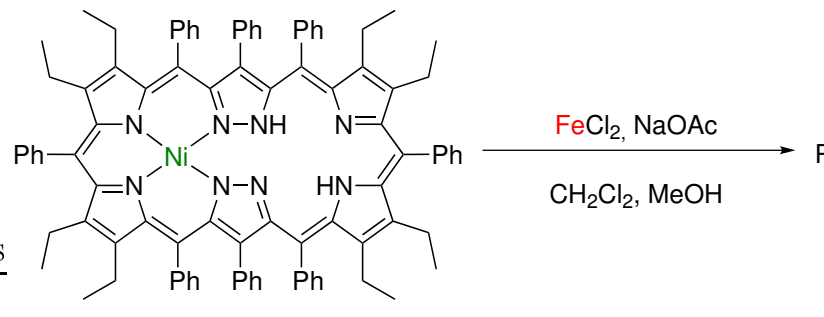

$\mathrm{LH}_{2} \mathrm{Ni}$

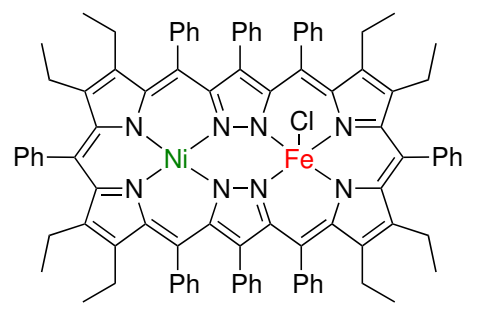

$\mathrm{LNiFeCl}$

Scheme 5.1: Synthesis of the ferric nickel iron complex LNiFeCl.

An excess of $\mathrm{FeCl}_{2}$ and $\mathrm{NaOAc}$ was added to a solution of $\mathbf{L H}_{\mathbf{2}} \mathbf{N i}$ in polar solvents and the mixture was stirred for 15 minutes at ambient conditions. After removing the solvent under reduced pressure, $\mathrm{CH}_{2} \mathrm{Cl}_{2}$ was added and the suspension was filtered to remove excess $\mathrm{FeCl}_{2}$ and $\mathrm{NaOAc}$. The filtrate was filtered over a plug of basic aluminum 
oxide, where $\mathbf{L N i F e C l}$ was the only fraction passing, and the solvent was removed under reduced pressure. The raw product was recrystallized from a mixture of $\mathrm{CH}_{2} \mathrm{Cl}_{2}$ and $n$-hexane to yield ferric $\mathbf{L N i F e C l}$ as brown needles.

\subsubsection{Characterization of $\mathrm{LNiFeCl}$}

The iron nickel complex $\mathbf{L N i F e C l}$ was characterized with standard analysis techniques like HRMS and UV-vis spectroscopy and the metal ions' oxidation and spin state could be determined with Mößbauer and EPR spectroscopy in combination with magnetic susceptibility measurements.

The formation of $\mathbf{L N i F e C l}$ could be confirmed in mass spectrometry. In $\mathrm{ESI}^{+}$mass spectrometry the ionized species $[\mathrm{M}]^{+}$without any axial ligand was observed on a routine basis (Fig. 5.1), like in all previously synthesized metal complexes of the Siamese-twin porphyrin. ${ }^{[10,53-55]}$

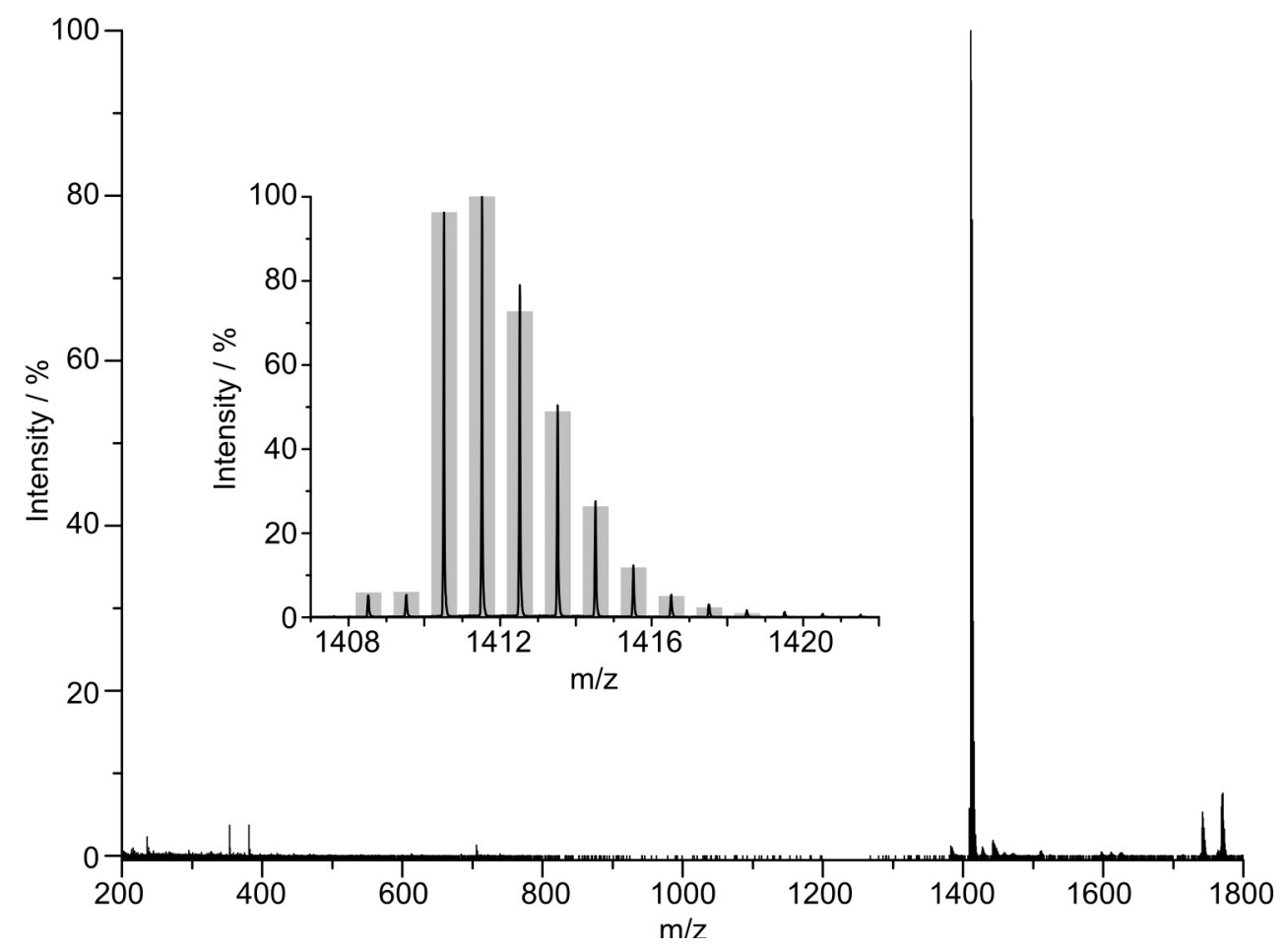

Figure 5.1: HRMS $\mathrm{ESI}^{+}$spectrum of crystalline $\mathbf{L N i F e C l}$ in MeOH. Inset: Comparison of the measured (black) and calculated isotopic pattern (grey bars). $[\mathrm{M}]^{+}$ $\mathrm{m} / \mathrm{z}=1410.5208$ (calcd.: 1410.5209).

The solid state structure of $\mathbf{L N i F e C l}$, as determined by single crystal X-ray diffraction, confirmed the expected oxidation of $\mathbf{L N i F e , ~ d u e ~ t o ~ a n ~ a x i a l ~ c o o r d i n a t i o n ~ o f ~ a ~ c h l o r i d e ~}$ (Fig. 5.2), that would most likely occur in a ferric complex.

In the molecular structure of $\mathbf{L N i F e C l}$ the $\mathrm{FeCl}$ moiety and the nickel atom are disordered about the two coordination sites of the ligand in a 1:1 ratio (1/2 occupancy) 


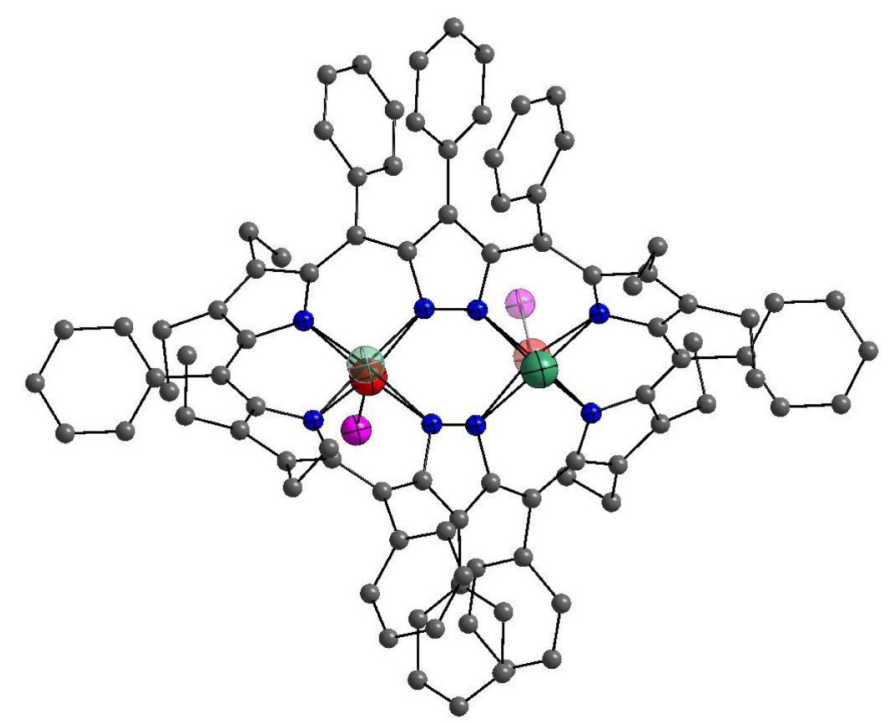

(a)

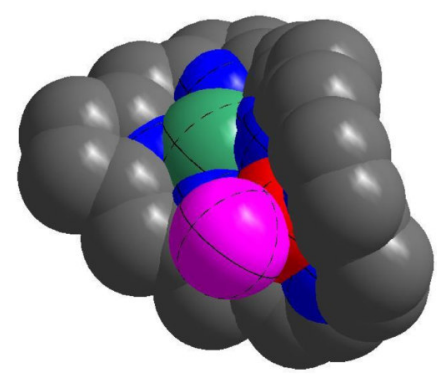

(b)

Figure 5.2: (a) Ball-and-stick model of the molecular structure of $\mathbf{L N i F e C l}$ and (b) spacefilling model of the sideview of the core-structure, without any substituent, of LNiFeCl , approximately along the iron nickel axis, determined by single crystal X-ray diffraction. All hydrogen atoms and solvent molecules were omitted for clarity (grey: carbon, blue: nitrogen, green: nickel, red: iron, pink: chlorine) For details, see Appendix.

(Fig. 5.2).

The optical spectrum of $\mathbf{L N i F e C l}$ showed the expected high absorption $\left(\epsilon=67400 \mathrm{M}^{-1} \mathrm{~cm}^{-1}\right)$ of the Soret like band $(380 \mathrm{~nm})$ and two additional Qbands (535 and $700 \mathrm{~nm}$ ) (Fig. 5.3) that are assumed to have metal contribution ${ }^{[53,86]}$ (see also Chap. 5.1.4).

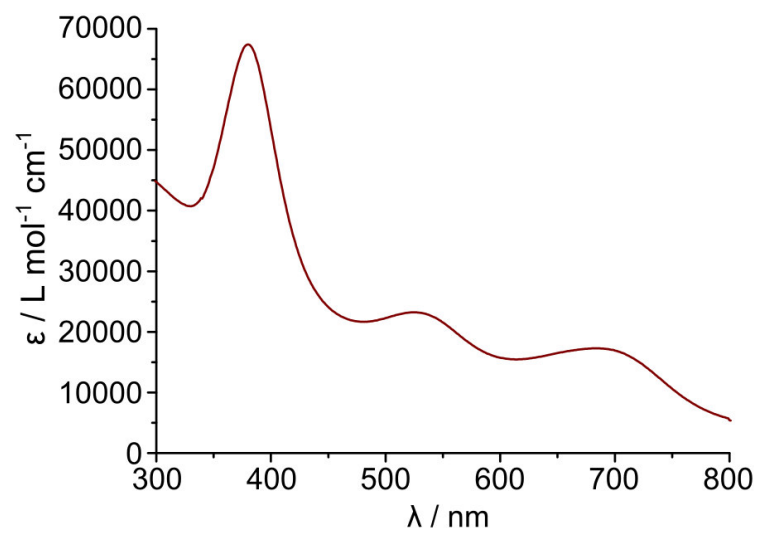

Figure 5.3: UV-vis spectrum of $\mathbf{L N i F e C l}$ in $\mathrm{CH}_{2} \mathrm{Cl}_{2}$ at ambient temperature.

To get further insights into the electronic structure of the heterobimetallic complex, magnetic susceptibility and EPR measurements were performed (Fig. 5.4).

The EPR measurements had to be done at $10 \mathrm{~K}$ due to the expected total spin of $\mathrm{S}>1 / 2$, which leads to signal broadening at higher temperatures. A total spin of $S>1 / 2$ is 


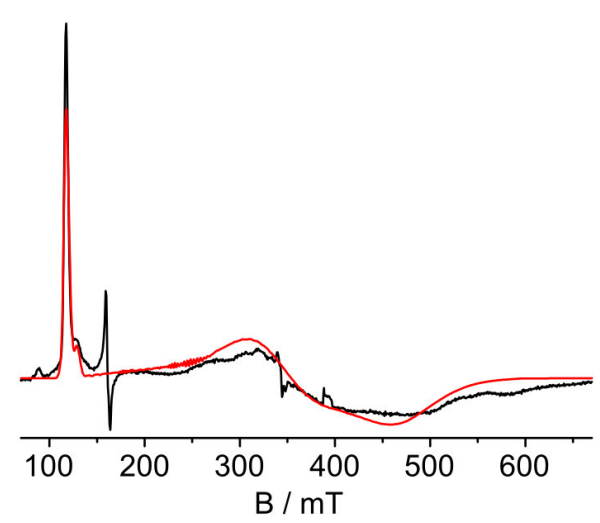

(a)

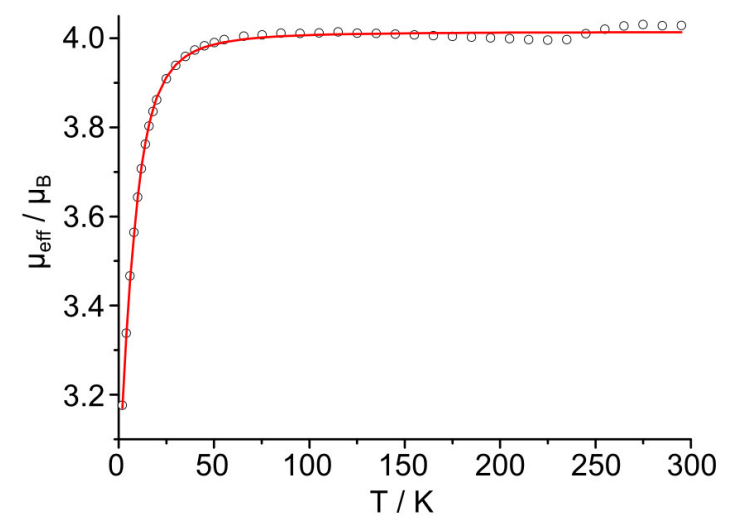

(b)

Figure 5.4: (a) X-band $\mathrm{EPR}$ spectrum of $\mathbf{L N i F e C l}$ in $\mathrm{CH}_{2} \mathrm{Cl}_{2}$ (frozen glass) at $10 \mathrm{~K}$ (black) and its simulation (red). Data were simulated with $\mathrm{g}_{x}=1.92, \mathrm{~g}_{y}=1.96, \mathrm{~g}_{z}=2.15$, (intrinsic line width $\mathrm{W}_{f}=14 \mathrm{mT}$ ), $\mathrm{D}=6.100$ and $\mathrm{E} / \mathrm{D}=0.33$. (b) Susceptibility measurement of $\mathbf{L N i F e C l}$ (black circles) and its fit (red) with $\mathrm{g}=2.073$, $\mathrm{D}=-9.981$ and $\mathrm{E} / \mathrm{D}=0.33$. The rhombic zero field splitting $(\mathrm{E} / \mathrm{D})$ was set to 0.33 like obtained from the EPR measurement.

expected because of the presumed iron ion's oxidation state of + III under non-inert conditions. The spin of the iron(III) ion can either be $\mathrm{S}=1 / 2$ (low spin (ls)), $\mathrm{S}=3 / 2$ (intermediate spin (is)) or $\mathrm{S}=5 / 2$ (high spin (hs)), whereby a low spin state in a rhombic square pyramidal complex with an anionic axial ligand has never been observed before. ${ }^{[8]}$ Furthermore, the spin state of the nickel(II) ion is assumed to be low spin ( $\mathrm{S}=0$ ), like observed in all nickel complexes of the Siamese-twin porphyrin. ${ }^{[54,55]}$ A total spin of more than $1 / 2$ is indeed the case (Fig. 5.4 (a)), as can be inferred from the EPR spectrum. The signal at $117 \mathrm{mT}$ and its shape are typical for an $\mathrm{S}=3 / 2$ system with large rhombic zero field splitting parameter. ${ }^{[119-121]}$ The shift towards high g-values is justified by the maximal rhombic zero field splitting of $\mathrm{E} / \mathrm{D}=0.33$. The actual $\mathrm{g}$-values are close to $\mathrm{g}=2$. The sharp signal at $160 \mathrm{mT}$, which integrates to less than $0.1 \%$, results from some iron impurity.

A total spin of $\mathrm{S}=3 / 2$ could be confirmed in magnetic susceptibilty measurements (Fig. 5.4); experimental data could be fitted using the isotropic Heisenberg-Dirac-Van-Vleck Hamiltonian corrected with the corresponding Zeeman term (equation (1)) with $B$ being the applied field and $\mu_{B}$ being the Bohr magneton. ${ }^{[122]}$ The effective magnetic moment $\mu_{\text {eff }}$ of a dinuclear complex can be determined with equation (2) as a spin-only value, ignoring coupling with $g$ being the Landé factor. $g$ has a value of 2.0023 for the free electron and is close to this value for most organic radicals but differs for transition metal ions due to larger spin-orbit coupling. ${ }^{[123-126]}$

$$
\hat{H}=-2 J \hat{S}_{1} \cdot \hat{S}_{2}+g \mu_{B}\left(\hat{S}_{1}+\hat{S}_{2}\right) B
$$




$$
\frac{\mu_{e f f}}{\mu_{B}}=g \sqrt{S_{1}\left(S_{1}+1\right)+S_{2}\left(S_{2}+1\right)}
$$

An effective magnetic moment of $\mu_{\text {eff }}=4.01$ fits to a $\mathrm{S}=3 / 2$ spin system, whose spin-only value would be 3.88 assuming a $g$-value of 2.0023 .

A spin system of $\mathrm{S}=3 / 2$ can be the result of an is-iron(III) center $(\mathrm{S}=3 / 2)$, of antiferromagnetic coupling between a hs-iron(II) $(\mathrm{S}=2)$ and an organic radical $(\mathrm{S}=1 / 2)$ or of antiferromagnetic coupling between a hs-iron(III) $(\mathrm{S}=5 / 2)$ and a hs-nickel(II) $(\mathrm{S}=1)$. The latter is extremely unlikely, because a hs-nickel(II) has never been observed in nickel complexes of the Siamese-twin porphyrin and is rather untypical for a square planar coordinated $\mathrm{d}^{8}$ nickel(II) ion. Within a square planar coordination environment, especially with strong binding ligands, the $d_{x^{2}-y^{2}}$ orbital is highly destabilized, leading to a low spin complex for $\mathrm{d}^{8}$ metals.

To determine the iron ion's oxidation and spin state in more detail, Mößbauer (MB) spectroscopy was used in solid state. The Mößbauer spectrum of $\mathbf{L N i F e C l}$ showed two doublets in a ratio of 20:80 with isomer shifts of $\delta_{F e}=0.53$ and 0.58 and a quadrupole splitting of $\Delta E_{Q}=1.20$ and 2.70, respectively (Fig. 5.5).

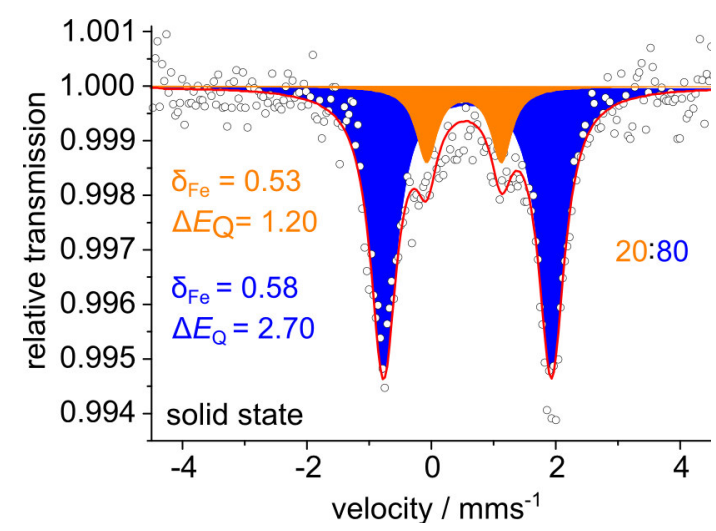

Figure 5.5: Solid state Mößbauer spectrum of $\mathbf{L N i F e C l}$ with $\delta_{F e}=0.53$ and $0.58, \Delta E_{Q}=1.20$ and 2.70 at $80 \mathrm{~K}$.

The presence of two doublets indicated two different iron ions within the material measured with distinct coordination environments with a big difference in $\Delta E_{Q}$. Whereas the doublet marked in blue is in agreement with the EPR and SQUID data, indicating an intermediate spin iron(III), the doublet marked in orange is indicative of a high spin iron(III), due to the isomer shift and the small quadrupole splitting of $\Delta E_{Q}=1.20$ (Tab. 5.1). An intermediate spin or a spin admixture of $\mathrm{S}=3 / 2$ and $5 / 2$ is well known from iron porphyrin systems, and the amount of each spin state strongly depends on the electronic structure of the porphyrin and especially the nature of the axial ligand. ${ }^{[82,127]}$ For example the first iron porphyrins with an is-Fe(III) ion have been synthesized with weakly binding axial ligands like $\mathrm{ClO}_{4}{ }^{-}$(Tab. 5.1). [128-130] 
Table 5.1: Mößbauer parameter of a selection of iron(III) porphyrins with $\mathrm{S}=3 / 2$ or $\mathrm{S}=5 / 2$ spin states at $80 \mathrm{~K}$.

\begin{tabular}{l|c|c|c|c}
\hline Complex & $\mathrm{S}$ & $\delta_{F e} / \mathrm{mms}^{-1}$ & $\Delta E_{Q} / \mathrm{mms}^{-1}$ & Ref. \\
\hline TPPFeCl $^{[\mathrm{a}]}$ & $5 / 2$ & 0.47 & 0.60 & {$[131]$} \\
$\mathrm{TPPFeClO}_{4}{ }^{[\mathrm{b}]}$ & $3 / 2$ & 0.38 & 3.48 & {$[132]$} \\
$\mathrm{OEPFeClO}_{4}{ }^{[\mathrm{c}]}$ & $3 / 2$ & 0.40 & 3.54 & {$[129]$} \\
$\mathrm{OETPPFeCl}^{[\mathrm{d}]}$ & $5 / 2$ & 0.35 & 0.95 & {$[133]$} \\
{$\left[\mathrm{OETPPFe}(\mathrm{THF})_{2}\right] \mathrm{ClO}_{4}$} & $3 / 2$ & 0.25 & 2.29 & {$[134]$} \\
{$\left[\mathrm{OETPPFe}(\mathrm{py})_{2} \mathrm{ClO}_{4}\right.$} & $3 / 2$ & 0.57 & 3.03 & {$[134]$} \\
\hline
\end{tabular}

TPP: meso-tetraphenyl-porphyrinato, OEP: $\beta$-octaethyl-porphyrinato, OETPP: $\beta$-octaethylmeso-tetraphenyl-porphyrinato.

An intermediate spin for Fe(III) is favored when one $d$-orbital lies strikingly higher in energy than the other four. Such an orbital arrangement occurs for example in $\mathrm{C}_{2 \mathrm{v}}$ or $\mathrm{C}_{4 \mathrm{v}}$ symmetric square-pyramidal Fe(III) complexes, when the $d_{x^{2}-y^{2}}$-orbital is highly destabilizied due to strong binding equatorial ligands and the $d_{z^{2}}$-orbital stays low in energy, because of a weakly binding axial ligand (Fig. 5.6). A spin of $S=3 / 2$ for a ferric complex is further favored when the porphyrin pocket is contracted or not exactly planar and/or the metal ion is coordinated more out-of-plane. ${ }^{82,117,135]}$

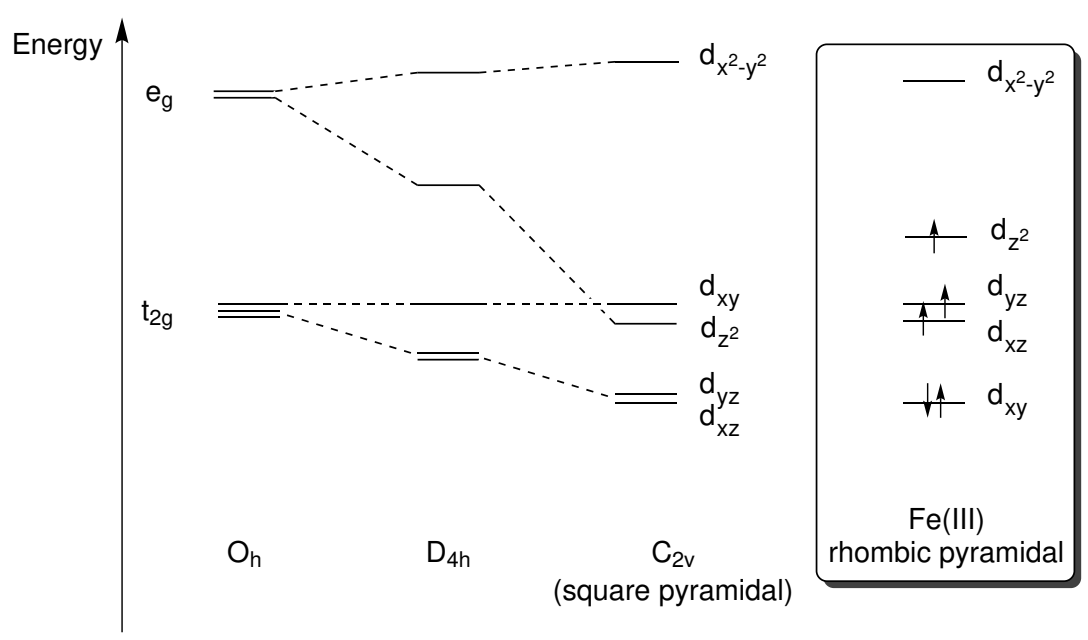

Figure 5.6: Schematic illustration of the splitting of molecular $d$-orbitals in different coordination environments and the favored $\mathrm{S}=3 / 2$ spin state in rhombic pyramidal Fe(III) complexes (black box). ${ }^{[82]}$

All these requirements for an intermediate spin iron(III) center seem to be fulfilled for LNiFeCl. With the Siamese-twin porphyrin's unique coordination motif in combination with the weakly binding chloride in the iron ion's axial position, splitting of the molecular orbitals is within the perfect range to favor the $\mathrm{S}=3 / 2$ intermediate spin state. However, if the two doublets in Figure 5.5 result from a spin admixture of $\mathrm{S}=3 / 2$ and $5 / 2$, this admixture should be temperature dependent, resulting in a non-constant effective magnetic moment. However, $\mu_{\text {eff }}$ is constant between 50 and $300 \mathrm{~K}$ (Fig. 5.4 
(b)) and clearly shows an $\mathrm{S}=3 / 2$ system over the whole temperature range.

To rule out that different coordination motifs in solid state, like in standard Mößbauer measurements, and frozen solution, like in the measured EPR spectrum, influence the spin state of the iron(III) center, EPR measurements in solid state and Mößbauer measurements in frozen solution were performed. An EPR measurement on solid material resulted in signal broadening (see. Appendix A31), which made interpretation difficult. Mößbauer measurements on frozen solutions were notably easier. Due to the fact, that Mößbauer spectroscopy only detects the ${ }^{57} \mathrm{Fe}$ isotope, which has a natural abundance of $2.18 \%,{ }^{[82]}$ the amount of substance needed for solution measurements is too high to be dissolved in any organic solvent suitable for Mößbauer spectroscopy, especially because measurements in frozen solution result in additional line broadening. ${ }^{[82,136]}$ Therefore the ${ }^{57}$ Fe labeled pendant $\mathbf{L N i}{ }^{57} \mathbf{F e C l}$ was synthesized by use of labeled ${ }^{57} \mathrm{FeCl}_{2}$.

The labeled iron(II) chloride was synthesized by the addition of concentrated $\mathrm{HCl}$ to metallic ${ }^{57} \mathrm{Fe}$ under inert conditions. The reaction mixture was stirred over night and the remaining aqueous $\mathrm{HCl}$ solution was removed under reduced pressure yielding white ${ }^{57} \mathrm{FeCl}_{2}$ powder. The synthesis of $\mathbf{L N i}{ }^{57} \mathbf{F e C l}$ was done as previously described for LNiFeCl.

To exclude the effect of solvent coordination, Mößauer measurements in coordinating THF and non-coordinating toluene were performed (Fig. 5.7).

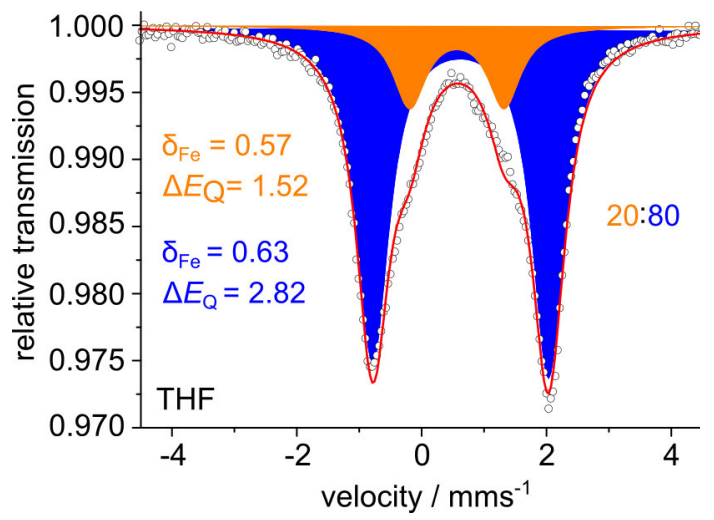

(a)

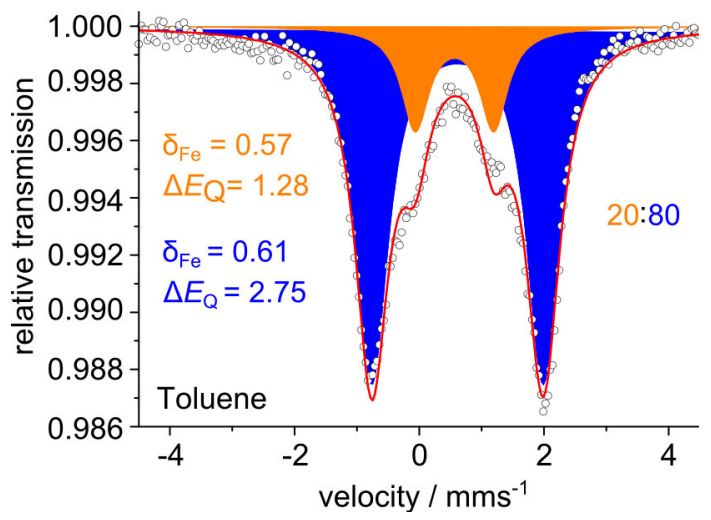

(b)

Figure 5.7: (a) Mößbauer spectrum of isotopically labeled $\mathbf{L N i}{ }^{57} \mathbf{F e}$ in THF $\left(\delta_{F e}=0.57\right.$ and $0.63, \Delta E_{Q}=1.52$ and 2.82) and (b) in toluene $\left(\delta_{F e}=0.57\right.$ and $0.61, \Delta E_{Q}=1.26$ and 2.75 ) both at $80 \mathrm{~K}$.

The Mößbauer measurements on frozen solutions clearly retained the ratio of the two doublets of 20:80 as observed in the solid state spectra (Fig. 5.5). The isomer shift as well as the quadrupole splitting and the line width slightly change but are in the region expected due to solvation. ${ }^{[82,136]}$ The presence of the inner Mößbauer doublet is highly interesting but could not be explained so far. Due to EPR and magnetic susceptibility measurements the inner signal seems to be an intermediate spin signal, with an unusual small isomer shift. This disagrees with a spin admixture. Additionally, 
if the iron center would be within the same coordination environment with only the spin varying, the high spin signal always has to have a higher isomer shift than the intermediate spin signal. ${ }^{[82,137]}$ This is not the case for $\mathbf{L N i F e C l , ~ f u r t h e r ~ i n d i c a t i n g ~}$ that the inner signal corresponds to an intermediate spin signal too, or does not belong to the molecule but to an impurity. However, an iron containing impurity could be ruled out, due to the use of crystalline and polycrystalline material and an elemental analysis, indicating no impurity, but the incorporation of two $n$-hexane and $0.75 \mathrm{CH}_{2} \mathrm{Cl}_{2}$ molecules per metal complex (see Chap. 5.1.1). Furthermore, investigations of the redox chemistry of $\mathbf{L N i F e C l}$ have shown a clean reduction towards ferrous $\mathbf{L N i F e}$, resulting in an spectrum giving only one Mößbauer doublet, further indicating the unlikeliness of an iron containing impurity. Intriguingly, the second inner doublet reappears after oxidation of the ferrous complex (Chap. 5.1.4).

The redox chemistry of $\mathbf{L N i F e C l}$ was studied by cyclic and square wave voltammetry and showed the expected two reversible ligand based oxidation events. Reversibility can be determined based on the shape of the wave and the separation between the oxidation and reduction half-wave. Furthermore, an additional irreversible reduction event was observed (Fig. 5.8) in contrast to the nickel and copper complexes of the parent STP (cf. Fig. 1.8).[55]

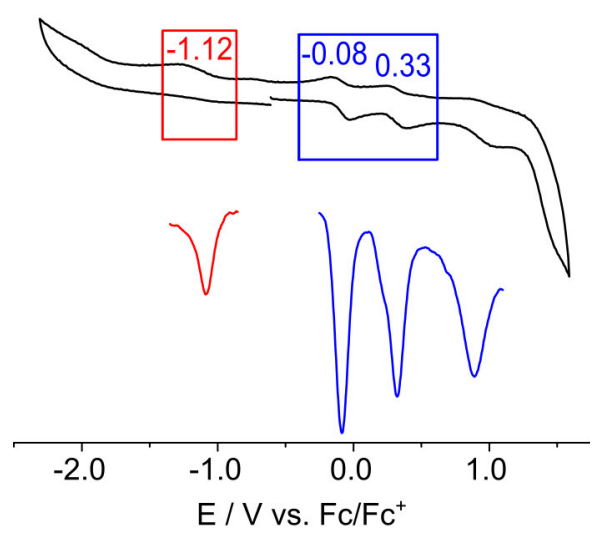

(a)

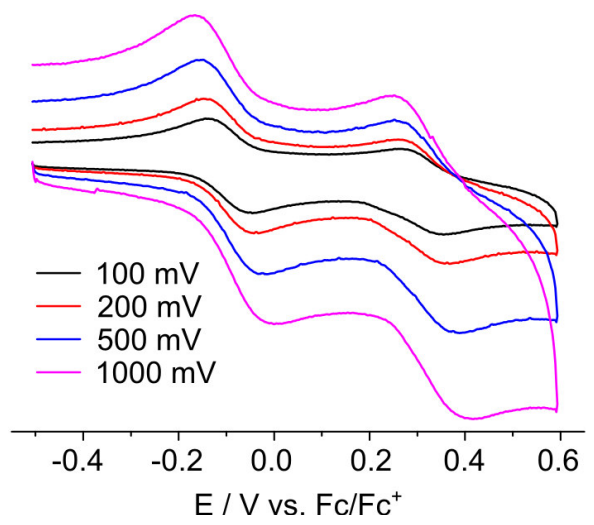

(b)

Figure 5.8: (a) CV (top) SWV (bottom) curve of $\mathbf{L N i F e C l}$ at a scan rate of $100 \mathrm{mV}$. The two reversible ligand-based oxidations are marked in blue and the assumed iron(III) reduction in red. Half wave potentials $\left(\mathrm{E}_{1 / 2}\right)$ are indicated in volts and determined from square wave voltammetry. (b) CV curve of the electrochemically reversible oxidations of $\mathbf{L N i F e C l}$ at different scan rates indicated. Both measurements were performed in $\mathrm{CH}_{2} \mathrm{Cl}_{2}$ at ambient temperature with $0.1 \mathrm{M}\left[\mathrm{Bu}_{4} \mathrm{~N}\right] \mathrm{PF}_{6}$ as electrolyte referenced to the redox couple $\mathrm{Fc} / \mathrm{Fc}^{+}$.

The two reversible ligand based oxidation waves at -0.08 and $+0.33 \mathrm{~V}$ vs. $\mathrm{Fc} / \mathrm{Fc}^{+}$and the reduction wave at around $-1.9 \mathrm{~V}$ are comparable with the measured values for the other metal complexes of the Siamese-twin porphyrin (Tab. 5.2). The additional reduction event at $-1.12 \mathrm{~V}$ vs. $\mathrm{Fc} / \mathrm{Fc}^{+}$was assigned to the reduction $\mathrm{Fe}(\mathrm{III}) / \mathrm{Fe}(\mathrm{II})$, a reduction that was not observable in nickel and copper complexes due to their already 
reduced oxidation state of $+\mathrm{II}$. The reduction potential of $-1.12 \mathrm{~V}$ vs. $\mathrm{Fc} / \mathrm{Fc}^{+}$for the redoxcouple $\mathrm{Fe}(\mathrm{III}) / \mathrm{Fe}$ (II) is in line with the observed potentials in the corresponding [meso-tetrakis(aryl)porphyrinato]-iron(III) and [ $\beta$-octalkylporphyrinato]-iron(III) complexes (Tab. 5.2).

Table 5.2: Potentials of the first reduction and the first two oxidations of $\mathbf{L N i F e C l}{ }^{[a]}$ in comparison to the nickel and copper complexes of the STP and the corresponding [meso-tetrakis(aryl)porphyrinato]-iron(III) and [ $\beta$-octalkylporphyrinato]iron(III) complexes vs. $\mathrm{Fc} / \mathrm{Fc}^{+}$.

\begin{tabular}{|c|c|c|c|c|c|}
\hline Complex & $\begin{array}{c}E \text { Red / V } \\
\text { ligand }\end{array}$ & $\begin{array}{c}E \operatorname{Red} / \mathrm{V} \\
\text { metal }\end{array}$ & $E$ Ox $1 / \mathrm{V}$ & $E$ Ox $2 / \mathrm{V}$ & Ref. \\
\hline $\begin{array}{l}\mathbf{L N i F e C l} \\
\mathbf{L N i}_{2}{ }^{[c]} \\
\mathbf{L C u N i}^{[c]} \\
\mathbf{L C u}_{2}{ }^{[c]}\end{array}$ & $\begin{array}{c}-1.92 \\
-1.90 \\
-1.81^{[\mathrm{b}]} \\
-1.86\end{array}$ & $\begin{array}{c}-1.12^{[\mathrm{b}]} \\
- \\
- \\
-\end{array}$ & $\begin{array}{l}-0.08 \\
-0.16 \\
-0.34 \\
-0.37\end{array}$ & $\begin{array}{c}0.33 \\
0.30 \\
0.24 \\
-0.02\end{array}$ & $\begin{array}{c}- \\
{[55]} \\
{[55]} \\
{[55]}\end{array}$ \\
\hline $\begin{array}{l}\text { TPPFeCl }^{[\mathrm{d}, \mathrm{e}]} \\
\mathrm{OEPFeCl}^{[\mathrm{e}, \mathrm{f}]} \\
\mathbf{E t}_{\mathbf{8}} \mathrm{TPPFeCl}^{[\mathrm{e}, \mathrm{g}, \mathrm{h}]}\end{array}$ & $\begin{array}{l}- \\
- \\
-\end{array}$ & $\begin{array}{l}-0.75 \\
-0.98 \\
-1.06\end{array}$ & $\begin{array}{l}0.68 \\
0.55 \\
0.20\end{array}$ & $\begin{array}{l}0.94 \\
0.93 \\
0.66\end{array}$ & $\begin{array}{l}{[138]} \\
{[139]} \\
{[140]}\end{array}$ \\
\hline
\end{tabular}

[a] Values as determined by square wave voltammetry (in $\mathrm{CH}_{2} \mathrm{Cl}_{2}, 0.1 \mathrm{M}\left[\mathrm{Bu}_{4} \mathrm{~N}\right] \mathrm{PF}_{6}$ ) cf. to Fig. 5.8. [b] Electrochemically irreversible. [c] The values reported previously ${ }^{[55]}$ have been incorrectly referenced because of conflicting data in literature. ${ }^{[141]} \mathrm{A}$ potential of $-0.48 \mathrm{~V}$ of decamethylferrocene vs. ferrocene was choosen instead of $-0.59 \mathrm{~V}$. A correction of $-0.11 \mathrm{~V}$ was done in this table. [d] [meso-tetraphenyl-porphyrinato]-iron(III)chloride. [e] Experimental data referenced vs. SCE and converted with $E\left(\mathrm{Fc} / \mathrm{Fc}^{+}\right)=0.46 \mathrm{~V}$ vs. SCE. ${ }^{[141]}[\mathrm{f}][\beta$-octaethyl-porphyrinato]iron(III)chloride. [g] [ $\beta$-octaethyl-meso-tetraphenyl-porphyrinato]-iron(III)chloride. [h] Different solvent: $\mathrm{PhCN}$.

With an increasing electron pushing effect of the substituents, the reduction is hampered and therefore shifted to a more negative potential, as observed for several other metal porphyrins. ${ }^{[56]} \mathbf{L N i F e C l , ~ e v e n ~ t h o u g h ~ t h e ~ S T P ~ i s ~ e l e c t r o n i c a l l y ~ m u c h ~ d i s t i n c t ~ f r o m ~ a ~}$ regular porphyin, it fits into this series of redox potentials (Tab. 5.2).

In contrast to the two oxidations, the reduction of $\mathrm{Fe}(\mathrm{III})$ in $\mathbf{L N i F e C l}$ is electrochemically irreversible (Fig. 5.8 (a)). Irreversibility of the reduction of ferric porphyrins has been observed earlier and is usually a result of follow up chemical reactions. ${ }^{[140,142,143]}$ Furthermore, both the potential and reversibility of the redox events are dependent on the axial ligand and the solvent. ${ }^{[138,142-145]}$ In the case of $\mathbf{L N i F e C l}$ the irreversiblity can result from a decoordination of the axial chloride ligand upon reduction to the ferrous pendant LNiFe. Furthermore, the excess of $\mathrm{PF}_{6}{ }^{-}$could for example lead to the formation of a complexe like $[\mathbf{L N i F e}]^{+} \mathbf{P F}_{\mathbf{6}}{ }^{-}$during reoxidation.

Due to the irreversibility of the reduction wave at $-1.12 \mathrm{~V}$ electrochemical measurements with $\left[\mathrm{Bu}_{4} \mathrm{~N}\right] \mathrm{Cl}$ as electrolyte were performed to probe whether the reduction becomes reversible. Unfortunatelly suitable measurements were not possible, due to low signal intensity and a narrowed potential window. 
With distinct seperated oxidation and reduction waves, $\mathbf{L N i F e C l}$ is a suitable complex for reduction and oxidation chemistry, and the synthesis of the ferrous complex LNiFe should be easily accessible with the right reduction agent (see. Chap. 5.1.4).

\subsubsection{Oxidation of $\mathrm{LNiFeCl}$}

According to the oxidation of the copper and nickel complexes of the Siamese-twin porphyrin ${ }^{[55]}$ oxidation of $\mathbf{L N i F e C l}$ was performed. The determination of the oxidation locus could give insights into subsequent reactivity studies with both, the iron(II) and iron (III) complex of the STP, in which an oxidation of either the metal ion and/or the Siamese-twin porphyrin scaffold will take place.

The oxidation with $\mathrm{AgBF}_{4}$ was found to be the best way to oxidize the metal complexes of the Siamese-twin porphyrin (Fig. 5.2). ${ }^{[55,86]}$

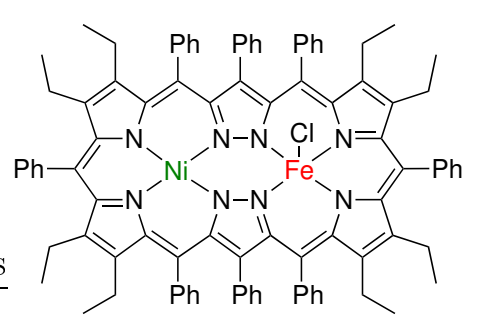

$\mathrm{LNiFeCl}$
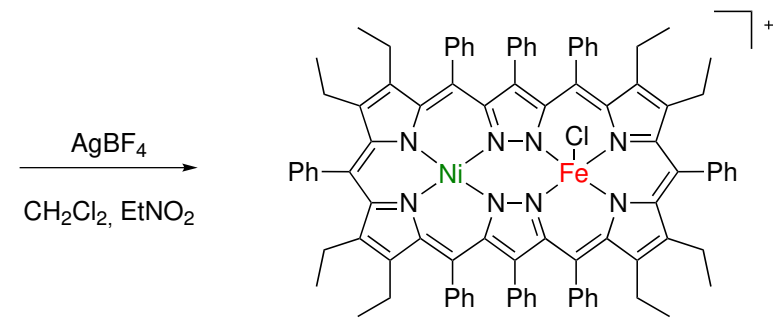

$\mathrm{LNiFeCl}^{+}$

Scheme 5.2: Oxidation of ferric nickel iron complex $\mathbf{L N i F e C l}$ with silver(I).

The ferric nickel iron complex $\mathbf{L N i F e C l}$ was dissolved in $\mathrm{CH}_{2} \mathrm{Cl}_{2}$ and treated with $\mathrm{AgBF}_{4}$, dissolved in nitroethane. Nitroethane was chosen as solvent, because $\mathrm{AgBF}_{4}$ is insoluble in $\mathrm{CH}_{2} \mathrm{Cl}_{2}$. Additionally, nitroethane does not have any absorption bands above $400 \mathrm{~nm}$. To get an insight into the oxidation process, UV-vis spectra, monitoring the transformation of $\mathbf{L N i F e C l}$ into the oxidized species, were recorded. Therefore, a solution of $\mathbf{L N i F e C l}$ was treated with 1.0, 2.0 and 2.5 equivalents of $\mathrm{AgBF}_{4}$. Like observed for the nickel and copper complexes, the intensity of the Soret like absorption band diminished, whereas the first Q-band, which has been assigned to have at least some metal character, is rising (Fig. 5.9).

After the addition of more than two equivalents of oxidizing agent $\mathrm{AgBF}_{4}, \mathbf{L N i F e C l}$ started to decompose. In comparison to chemical oxidation, electrochemical oxidation was performed, followed by simultaneous UV-vis spectroscopy (spectro-electrochemistry) (Fig. 5.9). Electrochemical oxidation was performed on the ferric complex LNiFeCl under inert conditions at +0.05 and $+0.4 \mathrm{~V}$ vs. $\mathrm{Fc} / \mathrm{Fc}^{+}$, because cyclic voltammetry has shown two suitable reversible oxidation waves at -0.08 and $+0.33 \mathrm{~V}$ vs. $\mathrm{Fc} / \mathrm{Fc}^{+}$ 


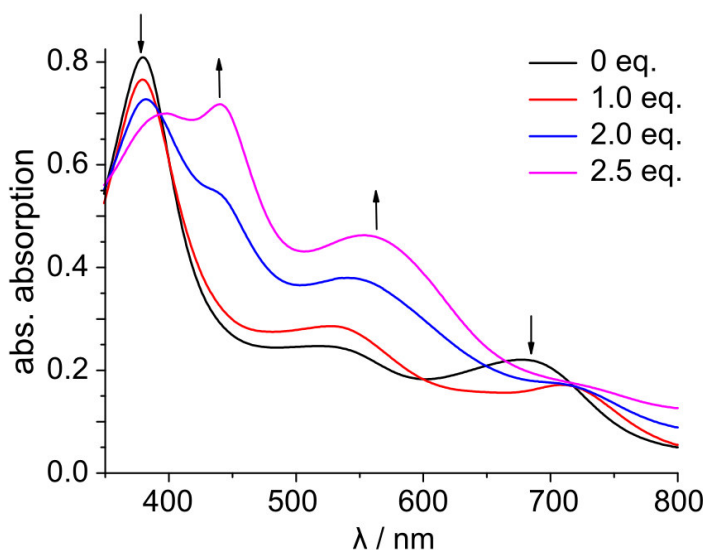

(a)

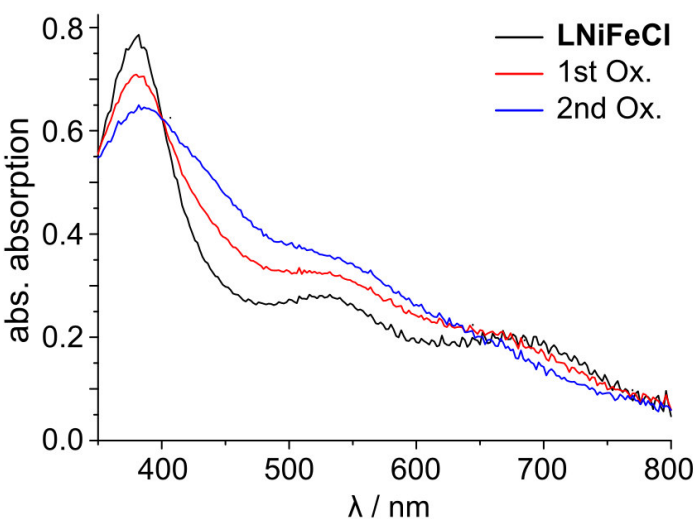

(b)

Figure 5.9: (a) UV-vis spectra of the chemical oxidation of LNiFeCl (black) with different equivalents of $\mathrm{AgBF}_{4}$ indicated in $\mathrm{CH}_{2} \mathrm{Cl}_{2}$ at ambient temperature under inert conditions. (b) UV-vis spectra of the electrochemical oxidation of $\mathbf{L N i F e C l}$ (black) at 0.05 (1st oxidation, red) and $0.40 \mathrm{~V}$ vs. $\mathrm{Fc} / \mathrm{Fc}^{+}$(2nd oxidation, blue) in $\mathrm{CH}_{2} \mathrm{Cl}_{2}$ at ambient temperature under inert conditions with $\mathrm{Bu}_{4} \mathrm{NPF}_{6}$ as eletrolyte.

(Fig. 5.8). The UV-vis spectrum showed again a conversion towards $\mathbf{L N i F e C l}{ }^{+}$and $\mathbf{L N i F e C l}^{2+}$, respectively. Because of the platinum net within the beam of the UV-vis spectrometer and due to very fast scans (every 5 seconds) the signal to noise ratio is affecting the intensity of each absorption band, especially in the Q-band region (Fig. 5.9).

To investigate the redox locus of the oxidations, EPR measurements were performed at liquid nitrogen temperature. Even though the $\mathrm{S}=3 / 2$ signal would only be detectable at lower temperatures, the ligand oxidation, which is commonly taking place upon oxidation on a regular basis, was detectable at a temperature of $133 \mathrm{~K}$ (Fig. 5.2).

EPR samples of oxidized $\mathbf{L N i F e C l}$ have been prepared according to the procedure of oxidized nickel and copper complexes. ${ }^{[55]}$ The ferric nickel iron complex $\mathbf{L N i F e C l}$ was dissolved in $\mathrm{CH}_{2} \mathrm{Cl}_{2}$ and one or two equivalents of $\mathrm{AgBF}_{4}$ dissolved in nitroethane were added, respectively. The solvent was removed under reduced pressure and the residue was redissolved in $\mathrm{CH}_{2} \mathrm{Cl}_{2}$ to achieve a concentration suitable for EPR measurements. The solution was filtered to remove elemental silver and directly frozen to avoid rereduction and/or decomposition and stored at $-80{ }^{\circ} \mathrm{C}$ in a freezer.

Even though UV-vis spectroscopy indicated two different species upon the first and second oxidation (Fig. 5.9), the EPR spectra of once and twice oxidized LNiFeCl look very similar. Only the ratio between the signal of the organic radical (at around $330 \mathrm{mT}$ ) and the $\mathrm{S}=3 / 2$ signal (at around $150 \mathrm{mT}$ ) differs in both oxidation states of the complex (Fig. 5.10). The oxidation of the ferric complex can take place at both dipyrromethane like sides of the Siamese-twin porphyrin (cf. Fig. 1.8). However, the side with the nickel(II) ion should be preferred, due to the already "oxidized" ferric side of the complex, where an equilibrium between a ligand and a metal based radical, 


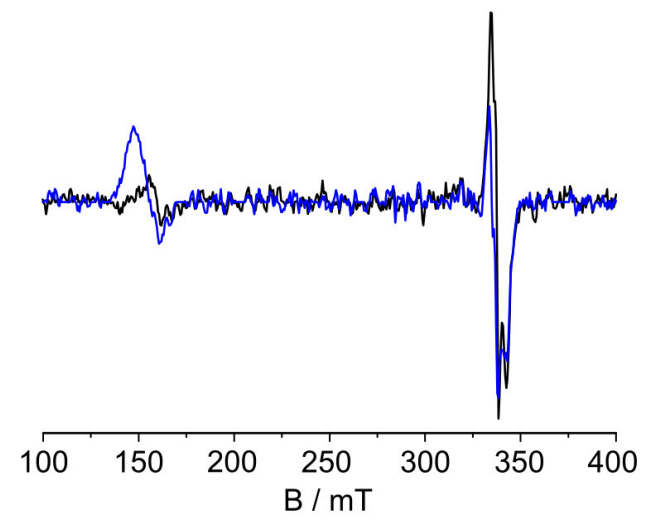

Figure 5.10: EPR spectra of chemically oxidized $\mathbf{L N i F e C l}^{+}$(black) and $\mathbf{L N i F e C l}^{2+}$ (blue) in $\mathrm{CH}_{2} \mathrm{Cl}_{2}$ at $133 \mathrm{~K}$.

resulting in a formerly iron(IV) ion, is most unlikely. This is in contradiction with the measured EPR spectra. Interestingly no nickel(III) signal was observed, neither in the once nor the twize oxidized complex, as observed for the previously synthesized nickel complexes of the Siamese-twin porphyrin, if the first oxidation took place on the nickel side (Chap. 6). ${ }^{[55,86]}$ Furthermore the addition of $\mathrm{Bu}_{4} \mathrm{NCl}$ did not lead to a vanishing of the signal for the organic radical, like it was observed in cobalt and nickel complexes of the STP (Chap. 6). This further indicates a different behavior of $\mathbf{L N i F e C l ~ i n ~ o x i d a t i o n ~}$ chemistry due to the incorporated iron(III) ion.

The oxidized complexes could not be isolated, due to rapid decomposition. While it has been shown that the twice oxidized complexes of $\mathbf{L} \mathbf{C u}_{\mathbf{2}}, \mathbf{L C u N i}$ and $\mathbf{L N i}_{\mathbf{2}}$ are unstable, ${ }^{[55,86]}$ also the once oxidized complex of $\mathbf{L N i F e C l}$ was decomposing. The reason might be the presence of the iron(III) ion. Thus, $\mathbf{L N i F e C l}$ contains a metal ion that already is in a higher oxidation state and is therefore prone to decompose even if only oxidized once further.

A possible instability of oxidized metal complexes of the Siamese-twin porphyrin is not unexpected, considering that two electron reactions in porphyrins can lead to either a decomposition or in the meso-positions subsituted derivatives even if no dioxygen is present. ${ }^{[8,32,146]}$ The mechanisms of the breakdowns and substitution reactions of metal porphyrins are not well understood so far. For that reason and because the electronic structure of the Siamese-twin porphyrin is notably different from the one of a regular porphyrin, no further statement on the decomposition in the oxidized metal complexes can be made for now. 


\subsubsection{Reduction of LNiFeCl}

The distinct isolated reduction event of $\mathbf{L N i F e C l ~ r e c o m m e n d s ~ i t s e l f ~ f o r ~ i t s ~ r e d u c t i o n ~}$ to ferrous LNiFe. Besides electrochemical reduction, a variety of different typical reductants for ferric iron porphyrins were used, for example sodium hydrogensulfide (NaHS), ethanethiol (EtSH), sodium dithionite $\left(\mathrm{Na}_{2} \mathrm{~S}_{2} \mathrm{O}_{4}\right)$, potassium superoxide $\left(\mathrm{KO}_{2}\right)$ and cobaltocene $\left(\mathrm{CoCp}_{2}\right) \cdot{ }^{[8,147-149]}$

To get first insights into the reduction of $\mathbf{L N i F e C l}$, electrochemical reduction was performed at a potential of $-1.2 \mathrm{~V}$ vs. $\mathrm{Fc} / \mathrm{Fc}^{+}$under inert conditions, since cyclic voltammetry has shown a suitable reduction wave at $-1.12 \mathrm{~V}$ vs. $\mathrm{Fc} / \mathrm{Fc}^{+}$(Fig. 5.8) whose irreversibilty was explained with a coordination change upon reoxidation (Chap. 5.1). A potential slightly lower was applied to assure complete reduction. The reaction was followed with simultaneous UV-vis spectroscopy (Fig. 5.11).

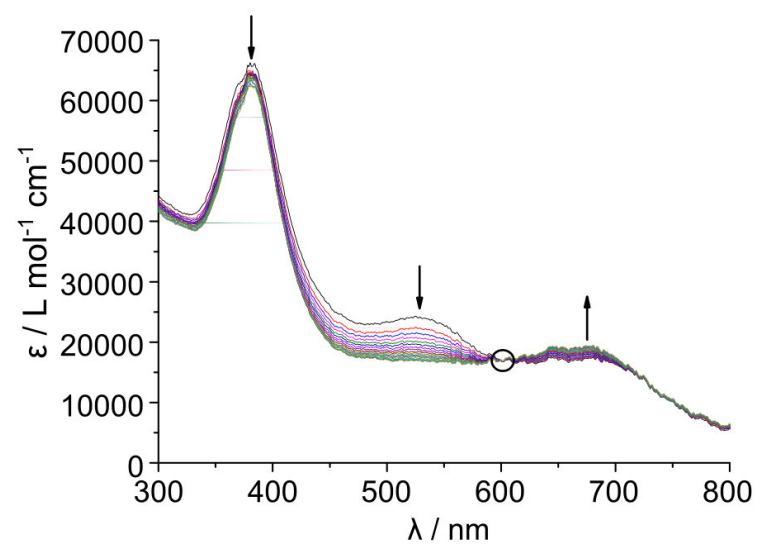

Figure 5.11: UV-vis spectrum of the electrochemical reduction of $\mathbf{L N i F e C l}$ in $\mathrm{CH}_{2} \mathrm{Cl}_{2}$ at ambient temperature with $\left[\mathrm{Bu}_{4} \mathrm{~N}\right] \mathrm{PF}_{6}$ as electrolyte at a potential $-1.2 \mathrm{~V}$ vs. the redox couple $\mathrm{Fc} / \mathrm{Fc}^{+}$. Data was collected every 5 seconds. Isosbestic points are marked with black circles

$\mathbf{L N i F e C l}$ gets rapidly reduced to $\mathbf{L N i F e}$ and the reduction is completed within 90 seconds. The reduction resulted in a color change from dark brown to green. The first Q-band at $535 \mathrm{~nm}$ vanishes, indicating this band to have some iron(III) contribution. Thus, this decline in absorption can be taken as a good indicator for the oxidation state of the iron nickel complex. Furthermore the isosbestic point (Fig. 5.11) indicates a clean conversion from one species into another. LNiFe showed rapid reoxidation in noncoordinating solvents like $\mathrm{CH}_{2} \mathrm{Cl}_{2}$, when the negative potential was not applied anymore. The iron(II) ion is within a rhombic coordination environment after reduction, due to the absence of a suitable neutral axial ligand. Such a coordination environment is unfavored for iron(II), leading to an instable complex. A stabilization was possible with the addition of a coordinating solvent like THF, but reoxidation still occurred rapidly, as could be observed in UV-vis spectroscopy. Mößbauer spectroscopy of the corresponding solu- 
tion, obtained from electrochemical reduction, was not suitable, due to the tremendous excess of electrolyte $\left[\mathrm{Bu}_{4} \mathrm{~N}\right] \mathrm{PF}_{6}$. Halides are known to absorb $\gamma$-radiation in Mößbauer spectroscopy, resulting in an enormous drop of the intensity. A cleanup of the solution of $\mathbf{L N i F e}$ was not possible due to immediate reoxidation, even under inert conditions as could be observed by UV-vis spectroscopy. To obtain a LNiFe solution suitable for MB spectroscopy, the former mentioned reductants (NaHS, EtSH, $\mathrm{Na}_{2} \mathrm{~S}_{2} \mathrm{O}_{4}, \mathrm{KO}_{2}$ ) were used on labeled ferric $\mathbf{L N i}{ }^{57} \mathbf{F e C l}$, to enhance the signal to noise ratio. The benefit of these reductants in comparison with for example $\mathrm{CoCp}_{2}$ is their demonstrated selectivity. Independent of the amount used, these reductants normaly reduce ferric porphyrin complexes only once. ${ }^{[8,147-149]}$ Obviously NaHS and EtSH are strong ligands, which result in decoordination of the iron ion in the case of $\mathbf{L N i F e C l}$, as could be observed by HRMS and UV-vis spectroscopy and excludes these as suitable reductants. However, with $\mathrm{Na}_{2} \mathrm{~S}_{2} \mathrm{O}_{4}$ and $\mathrm{KO}_{2}$ suitable reductants for $\mathbf{L N i F e C l}$ were found, as could be distinguished by UV-vis spectroscopy, which are easy to seperate from the solution by simple filtration. However with the addition of $\mathrm{Na}_{2} \mathrm{~S}_{2} \mathrm{O}_{4}$, decomposition was observed after filtration, which does not occur with $\mathrm{KO}_{2}$. Interestingly, potassium superoxide is suitable to reduce ferric porphyrins even though dioxygen is released, as long as an excess of the reducing agent is present or the reaction is performed in coordinating solvents, stabilizing the iron(II) species due to coordination. ${ }^{[148,150]}$ Nevertheless no MB spectrum from the resulting solution of $\mathbf{L N i}^{\mathbf{5 7}} \mathbf{F e}$ could be obtained so far.

With the stoichiometric use of $\mathrm{CoCp}_{2}$ the reduction of $\mathbf{L N i}^{57} \mathbf{F e C l}$ was successful and the resulting solution in THF could directly be used for MB spectroscopy (Fig. 5.12). $\mathrm{CoCp}_{2}$ has a potential of $-1.33 \mathrm{~V}$ vs. $\mathrm{Fc} / \mathrm{Fc}^{+},{ }^{[141]}$ which is in the perfect range for the reduction of the iron ion in $\mathbf{L N i F e C l}\left(\mathrm{E}_{1 / 2}=-1.12 \mathrm{~V}\right)$ and is only slightly lower than the potential of $-1.2 \mathrm{~V}$ applied in electrochemical reduction (Fig. 5.11).

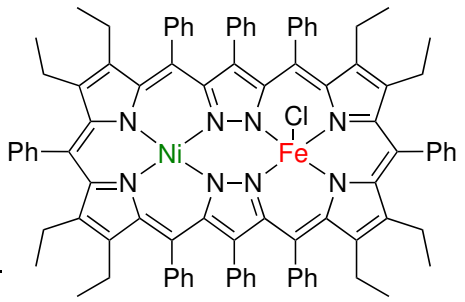

$\mathrm{LNiFeCl}$
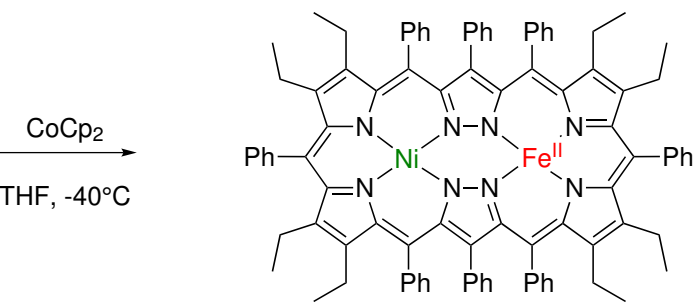

LNiFe

Scheme 5.3: Reduction of the ferric nickel iron complex $\mathbf{L N i F e C l}$ with cobaltocene at $-40{ }^{\circ} \mathrm{C}$.

A solution of $\mathrm{CoCp}_{2}$ in dry THF was added to a solution of $\mathbf{L N i F e C l}$ in dry THF under inert conditions at low temperature (Fig. 5.3). The solution was stirred for 5 minutes and directly used for the characterization and further reactions. The resulting UV-vis spectrum of the solution matched the one of the electrochemical reduction (see Appendix Fig. A33). For MB spectroscopy the corresponding labeled complex $\mathbf{L} \mathbf{N i}^{\mathbf{5 7}} \mathbf{F e C l}$ was used and the reaction mixture was transferred into a Mößbauer sample holder and directly frozen. 


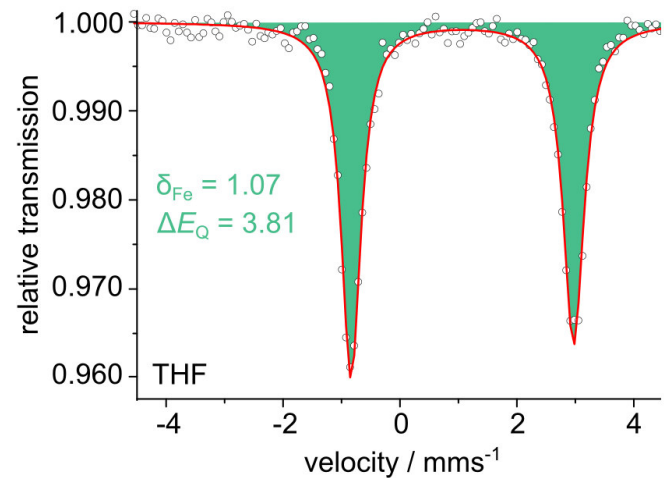

Figure 5.12: Mößbauer spectrum of labeled ferrous high spin $\mathbf{L N i}{ }^{57} \mathbf{F e}$ in $\mathrm{THF}$ at $80 \mathrm{~K}$ with $\delta_{F e}=1.07$ and $\Delta E_{Q}=3.81$.

The Mößbauer spectrum clearly shows a high spin iron(II) (Fig. 5.12), proving a successful reduction of $\mathbf{L N i F e C l}$. A high spin state iron(II) ion can be assigned, due to an isomere shift of more than $0.9 \mathrm{mms}^{-1}$. ${ }^{[82]}$ In solution the hs-iron(II) ion in LNiFe can either be in octahedral or a rhombic pyramidal geometry. Even though both coordination geometries are known from ferrous high spin iron porphyrins (Tab. 5.3), a rhombic pyramidal geometry is normally preferred. ${ }^{[32,147,150-153]}$

Table 5.3: Mößbauer parameter of a selection of [meso-tetraphenyl-porphyrinato]iron(II) (TPPFe) complexes with different spin states at $80 \mathrm{~K}$.

\begin{tabular}{l|c|c|c|c}
\hline Complex & $\mathrm{S}$ & $\delta_{F e} / \mathrm{mms}^{-1}$ & $\Delta E_{Q} / \mathrm{mms}^{-1}$ & Ref. \\
\hline TPPFe & 1 & 0.50 & 1.51 & {$[154]$} \\
TPPFe(2-MeHIm) $)^{[a]}$ & 2 & 0.92 & 2.26 & {$[154]$} \\
TPPFe(py) 2 & 0 & 0.40 & 1.15 & {$[155]$} \\
TPPFe(THF) 2 & 2 & 0.95 & 2.64 & {$[150]$} \\
\hline
\end{tabular}

[a] 2-MeHIm: 2-methyl-3- $H$-imidazole.

Interestingly, the inner Mößbauer doublet, which was always observed for ferric $\mathbf{L N i F e C l}$ (Fig. 5.5), vanished. This disappearance further indicates the inner signal (cf. Fig. 5.5) not to be an impurity, because an impurity would still give a Mößbauer signal after reduction, likely different to the one of $\mathbf{L N i F e}$.

Intriguingly, the inner double reappears when $\mathbf{L N i F e}$ is reoxidized by the addition of the oxidant $m$-CPBA (Fig. 5.13).

A solution of $m$-CPBA in THF was added to the solution of reduced $\mathbf{L N i F e}$, transfered into a MB sample holder and directly frozen like before. When a substoichiometric amount of the oxidant was added, a mixture of LNiFe (green) and LNiFeCl (blue and orange) was observed (Fig. 5.13 (a)), that turned into the typical two doublets regularly observed for $\mathbf{L N i F e C l}$ when the amount was increased up to one equivalent (Fig. 5.13 (b)). The previously observed ratio of 20:80 is nearly recovered (30:70). 


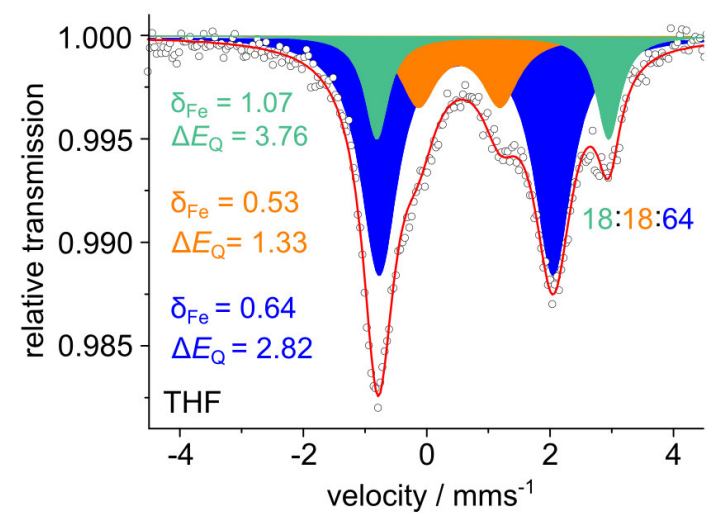

(a)

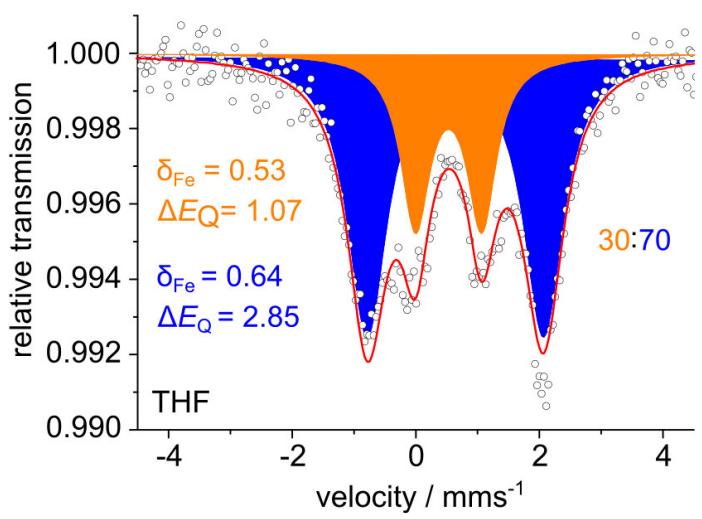

(b)

Figure 5.13: (a) Mößbauer spectrum of isotopically labeled ferrous high spin $\mathbf{L N i}^{57} \mathbf{F e}$ after reoxidation with substoichiometric $\left(\delta_{\mathrm{Fe}}=0.53,0.64\right.$ and $1.07 ; \Delta E_{Q}=1.33,2.82$ and 3.76) and (b) with stoichiometric amount of $m$-CPBA $\left(\delta_{F e}=0.53\right.$ and 0.64 ; $\Delta E_{Q}=1.07$ and 2.85.) in THF at $80 \mathrm{~K}$.

Table 5.4: Mößbauer parameter of $\mathbf{L N i F e}, \mathrm{LNiFeCl}$ and $\mathrm{LNiFe}$ after reoxidation with sub(a) and stoichiometric (b) amount of $m$-CPBA (cf. Fig. 5.15) in frozen THF solution at $80 \mathrm{~K}$.

\begin{tabular}{l|c|c|c}
\hline Complex & $\delta_{F e}$ & $\Delta E_{Q}$ & rel. Int. /\% \\
\hline LNiFe & 1.07 & 3.81 & 100 \\
\hline \multirow{2}{*}{ LNiFeCl } & 0.46 & 1.01 & 20 \\
& 0.54 & 2.79 & 80 \\
\hline \multirow{3}{*}{ Reoxidation (a) } & 1.07 & 3.76 & 18 \\
& 0.53 & 1.33 & 18 \\
& 0.64 & 2.82 & 64 \\
\hline \multirow{3}{*}{ Reoxidation (b) } & 0.53 & 1.07 & 30 \\
\hline
\end{tabular}

The slight differences in ratio, isomer shift and quadrupole splitting can be explained with possible side reactions and/or decomposition, supported by the loss in intensity from the partly reoxidized (Fig. 5.13 (a)) to the fully reoxidized spectrum (Fig. 5.13 (b)). If the reaction mixture was stirred for a longer time after the addition of $m$-CPBA, decomposition could be observed in UV-vis spectroscopy. The recovery of the two doublets indicates once more the inner doublet to be part of $\mathbf{L N i F e C l . ~}$

With ferrous LNiFe in hand, first preliminary reactivity studies were performed (Chap. 7) and the direct synthesis from the free base Siamese-twin porphyrin was performed (Chap. 5.1.5), due to the now known spectroscopic details and behavior of LNiFe. 


\subsubsection{Synthesis of ferrous LNiFe}

After the succesful synthesis of $\mathbf{L N i F e C l}$ and its structural characterization followed by spectroscopic characterization of its ferrous counterpart LNiFe, the latter one should be synthesized directly from the free base Siamese-twin porphyrin for later reactivity studies towards small molecule activation (Chap. 7).

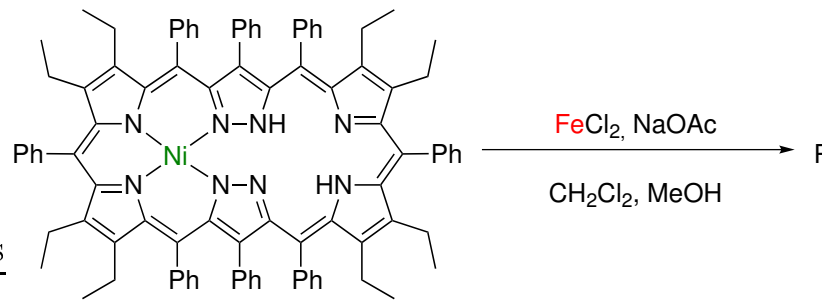

$\mathrm{LH}_{2} \mathrm{Ni}$

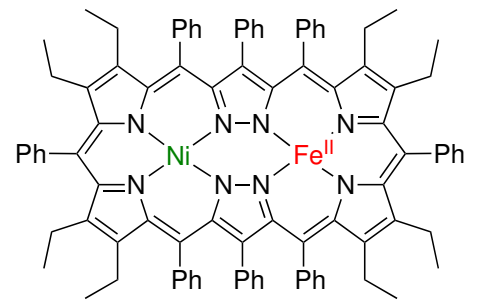

LNiFe

Scheme 5.4: Synthesis of the ferrous complex LNiFe.

LNiFe was synthesized according to the procedure of $\mathbf{L N i F e C l}$ (Chap. 5.1.1), but the reaction was carried out under inert conditions. Regardless, if the reaction was carried out under inert or non-inert condition the same ionized species $[\mathrm{M}]^{+}$without an axial ligand like for $\mathbf{L N i F e C l}$ was observed in inert and non-inert $\mathrm{ESI}^{+}$mass spectrometry. This indicates an already oxidized/ionized complex, which was observed for all previously synthesized copper(II) and nickel(II) complexes of the Siamese-twin porphyrin as well. Therefore ESI mass spectrometry is not suitable to distinguish between the oxidation states of the nickel iron complex. Nevertheless the color of the solution turned from greenish, typical for the ferrous complex, when synthesized under inert conditions, to brownish, typical for the ferric complex, when exposed to air. This indicated a successful synthesis of the ferrous complex LNiFe. Even though the solution was typically green, UV-vis spectroscopy showed a not negligible amount of already oxidized LNiFeCl due to the presence of the absorption band at $535 \mathrm{~nm}$ (Fig. 5.14).

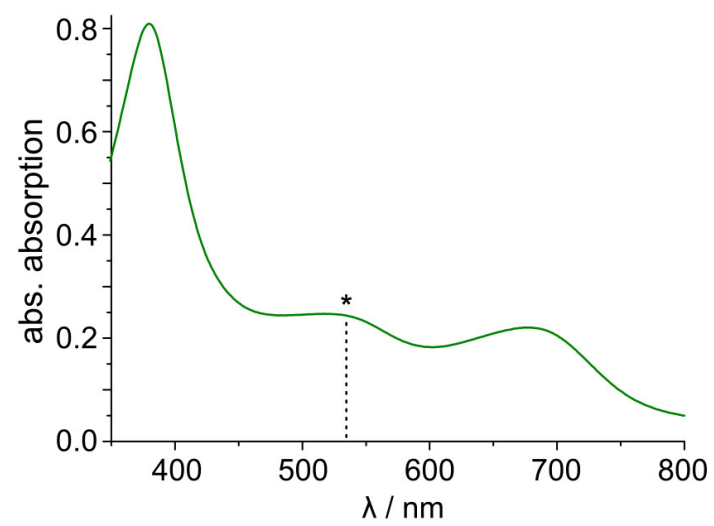

Figure 5.14: UV-vis spectrum of reaction mixture of the synthesis of $\mathbf{L N i F e}$ in $\mathrm{CH}_{2} \mathrm{Cl}_{2}$. The absorption band at $535 \mathrm{~nm}$, marked with a black asterisk, is characteristic for the oxidation state of the iron ion. 
The prominent Q-band at $535 \mathrm{~nm}$ is a good indicator for the oxidation state of the iron ion within the iron nickel complex $\mathbf{L N i F e}$ or $\mathbf{L N i F e C l . ~ W h e r e a s ~ t h i s ~ b a n d ~ i s ~ v e r y ~}$ intense in $\mathbf{L N i F e C l}$ it vanishes in the reduced form $\mathbf{L N i F e}$ (cf. Fig. 5.11).

Partial oxidation of LNiFe was furthermore confirmed by Mößbauer spectroscopy. The MB spectrum shows a mixture of the ferrous and ferric iron nickel complexes (cf. Fig. 5.13) together with an additional doublet typical for iron(II) (Fig. 5.15).

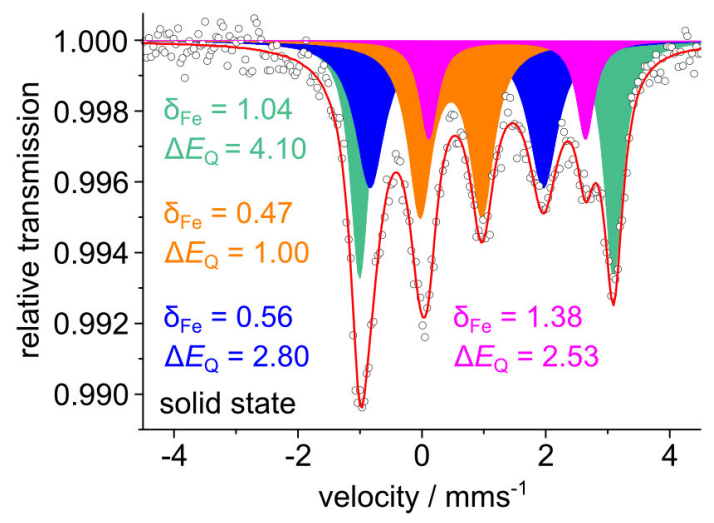

Figure 5.15: Mößbauer spectrum of partly oxidized $\mathbf{L N i F e}$ in $\mathrm{CH}_{2} \mathrm{Cl}_{2}$ at $80 \mathrm{~K}$ with $\delta_{F e}=0.47$, $0.56,1.04$ and $1.38 ; \Delta E_{Q}=1.00,2.80,4.10$ and 2.53 .

The additional doublet in Figure 5.15 with an isomer shift of $\delta_{F e}=1.38$ and a quadrupole splitting of $\Delta E_{Q}=2.53$ corresponds to a hs-iron(II) and is assumed to be remaining $\mathrm{FeCl}_{2}$, whose Mößbauer parameters are in good agreement with the one of the additional doublet (Tab. 5.4). ${ }^{[156,157]}$

Table 5.5: Mößbauer parameter of $\mathbf{L N i F e}$ in frozen THF solution, $\mathbf{L N i F e C l}$ and the reaction mixture during the direct synthesis of $\mathbf{L N i F e}(\mathrm{cf}$. Fig. 5.15) in solid state at $80 \mathrm{~K}$ in comparison to $\mathrm{FeCl}_{2}$ at $78 \mathrm{~K}$ in solid state (Fig.5.5).[156,157]

\begin{tabular}{l|c|c|c}
\hline Complex & $\delta_{F e}$ & $\Delta E_{Q}$ & rel. Int. /\% \\
\hline LNiFe & 1.07 & 3.81 & 100 \\
\hline \multirow{2}{*}{$\mathbf{L N i F e C l}$} & 0.53 & 1.20 & 20 \\
& 0.58 & 2.70 & 80 \\
\hline \multirow{4}{*}{ Reaction mixture } & 1.04 & 4.10 & 31 \\
& 0.47 & 1.00 & 28 \\
& 0.56 & 2.80 & 29 \\
$\mathrm{FeCl}_{2}$ & 1.38 & 2.53 & 12 \\
\hline
\end{tabular}

Partial oxidation of the ferrous complex could not be avoided, regardless which reaction conditions were chosen. Strikingly LNiFe could also not be stabelized with the addition of carbon monoxide, with the intention to form $\mathbf{L N i F e C O}$ or $\mathbf{L N i F e}(\mathbf{C O})_{\mathbf{2}},{ }^{[158]}$ a reaction that is well established in porphyrin chemistry to stabilize iron porphyrins with iron in the oxidation state of + II..$^{[152,158-161]}$ Instead, LNiFe decomposed over time, as 
could be observed in UV-vis spectroscopy. This decomposition was unexpected.

The oxidation of LNiFe is remarkable but not suprising, since the oxidation of $\mathrm{Fe}(\mathrm{II})$ to $\mathrm{Fe}(\mathrm{III})$ in $\mathbf{L N i F e}$ is easily accessible, as it was observed during electrochemical studies described in chapter 5.1.2 (cf. Fig. 5.8) and is much easier (lower potential) than in the corresponding TPP and OEP complexes (Tab. 5.2). Furthermore oxidation of iron porphyrin complexes under inert conditions has been observed before in several cases. ${ }^{[32,146]}$

Due to this oxidation, the synthesis of ferrous $\mathbf{L N i F e}$ has to be done starting from its ferric counterpart $\mathbf{L N i F e C l}$ followed by reduction with $\mathrm{CoCp}_{2}$ (Chap. 5.1.4).

\subsection{Mononuclear Iron Complex $\mathrm{LH}_{2} \mathrm{FeCl}$}

A mononuclear iron complex $\mathbf{L H}_{2} \mathbf{F e}$ of the Siamese-twin porphyrin is a suitable precursor for the synthesis of different heterobimetallic complexes incorporating redox active metal ions. With the combination of different metal ions within one complex, the redox properties can be selectively varied, which may help to find suitable complexes for the activation of small molecules.

A reaction temperature controlled monometallation resulting in $\mathbf{L H}_{2} \mathbf{F e}$ could not be achieved, even if only one equivalent of the metal salt, $\mathrm{FeCl}_{2}$ or $\mathrm{Fe}\left(\mathrm{BF}_{4}\right)_{2}$, was used (Fig. 5.5). Therefore, the solvent system for the complexation was changed from the previously used $\mathrm{CH}_{2} \mathrm{Cl}_{2} / \mathrm{MeOH}$ mixture to pyridine, according to recent observations by VOGEL. ${ }^{[16]}$ With the use of different iron(II) salts and different stoichiometry a suitable procedure for the synthesis of the monoiron complex $\mathbf{L H}_{\mathbf{2}} \mathbf{F e}$ was found.

Monometallation was possible with the use of $\mathrm{FeCl}_{2}$ in pure pyridine. However, monometallation could only be achieved with the right concentration of the free base porphyrin $\mathbf{L H}_{4}$, no matter which stoichiometry was used. Whereas a concentration below $1.5 \mathrm{~mm}$ did not yield any metallation or a mixture of $\mathbf{L H}_{\mathbf{4}}$ and $\mathbf{L H}_{\mathbf{2}} \mathbf{F e C l}$, a concentration above $1.5 \mathrm{~mm}$ resulted in a double metallation towards $\mathbf{L}\{\mathbf{F e C l}\}_{2}$. Even if only one equivalent of the metal salt was used, a mixture of the free base porphyrin $\mathbf{L H}_{\mathbf{4}}$, the monoiron and the diiron complex was observed.

Careful optimization of the reaction conditions finally led to the following protocol: $\mathrm{FeCl}_{2}$ was added to a $1.5 \mathrm{~mm}$ solution of the free base STP in pyridine and the reaction mixture was heated to $60{ }^{\circ} \mathrm{C}$ for 15 minutes under aerobic conditions. The solvent was removed under reduced pressure and toluene was added and removed again twice to eliminate excess pyridine. The residue was dissolved in toluene once again and the suspension was filtered to remove excess $\mathrm{FeCl}_{2}$. The resulting mixture of $\mathbf{L H}_{\mathbf{4}}, \mathbf{L}\{\mathbf{F e C l}\}_{\mathbf{2}}$ and $\mathbf{L H}_{2} \mathbf{F e C l}$ - a coordination of chloride in axial position was assumed similar to previously 


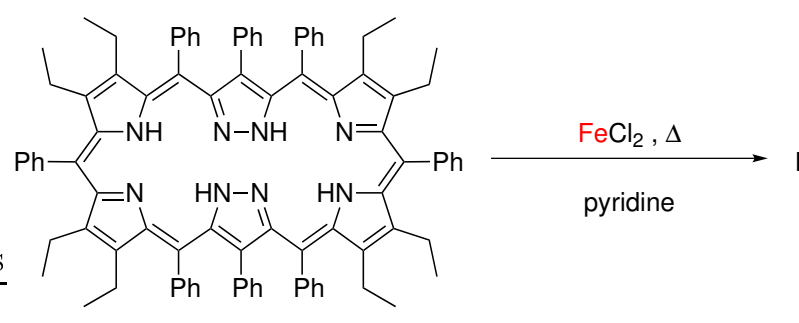

$\mathrm{LH}_{4}$

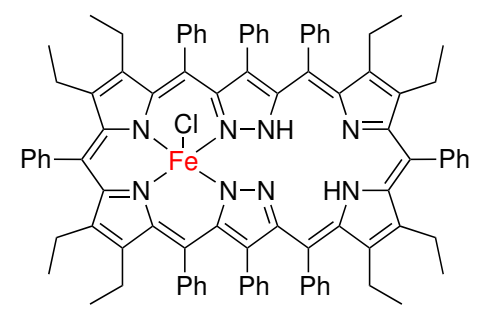

$\mathrm{LH}_{2} \mathrm{FeCl}$

Scheme 5.5: Synthesis of the monoiron complex $\mathbf{L H}_{2} \mathbf{F e C l}$.

synthesized $\mathbf{L N i F e C l ~ - ~ c o u l d ~ n o t ~ b e ~ p u r i f i e d ~ a n y ~ f u r t h e r ~ d u e ~ t o ~ i t s ~ i n s t a b i l i t y . ~ T h e ~}$ ferric monoiron complex showed decomposition during column chromatography and size selective chromatography could not seperate $\mathbf{L H}_{\mathbf{2}} \mathbf{F e C l}$ from $\mathbf{L} \mathbf{H}_{\mathbf{4}}$ and $\mathbf{L}\{\mathbf{F e C l}\}_{\mathbf{2}}$, due to their similar/same size. The mixture was therefore directly used for analysis and further reactions.

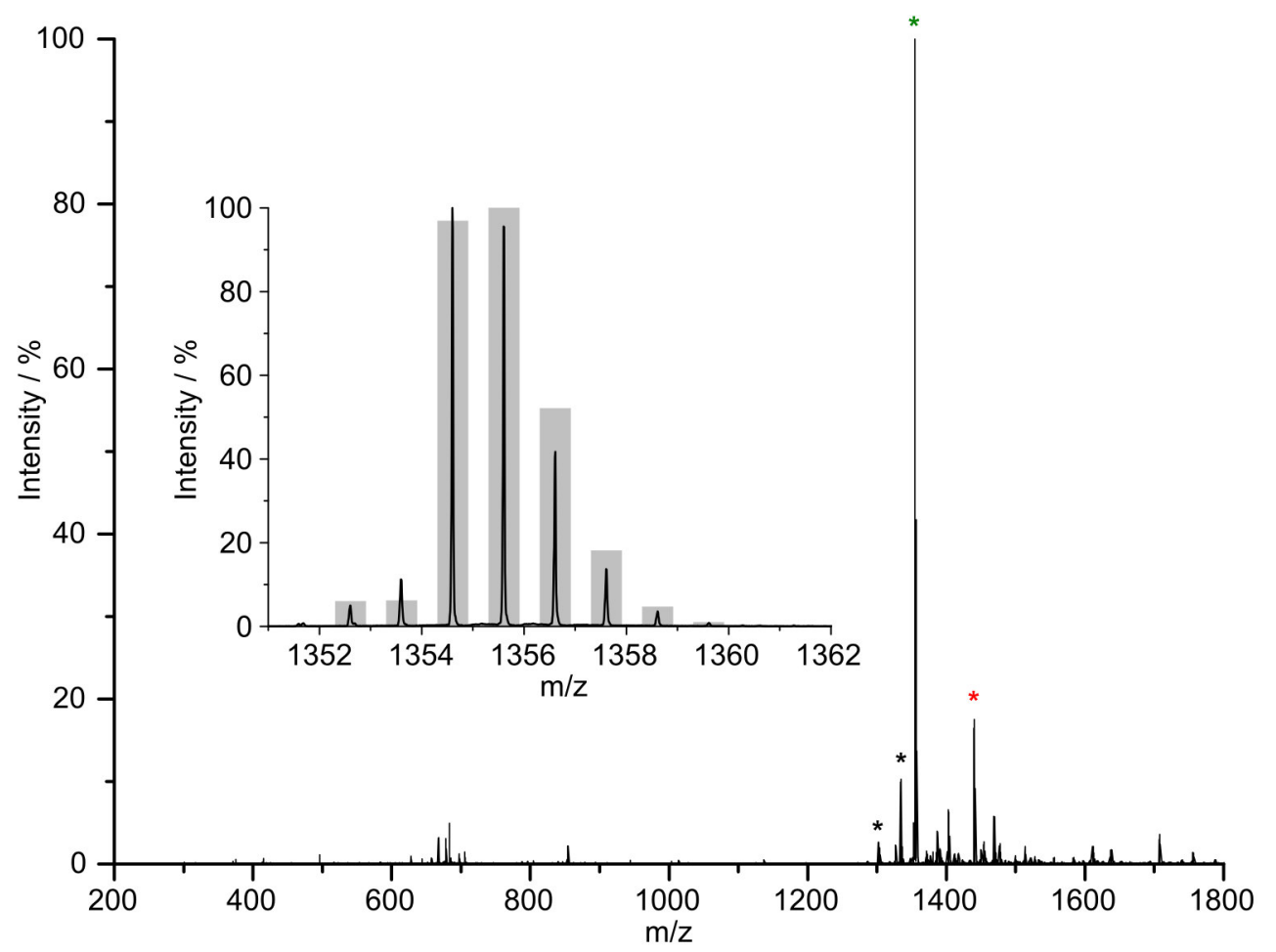

Figure 5.16: HRMS ESI ${ }^{+}$spectrum of the reaction mixture of $\mathbf{L H}_{2} \mathbf{F e}$ (marked with a green asterisk), $\mathbf{L}\{\mathbf{F e C l}\}_{2}\left([\mathrm{M}-\mathrm{Cl}+\mathrm{MeOH}]^{+}\right.$marked with a red asterisk) and $\mathbf{L H}_{4}$ $\left(\left[\mathbf{L H}_{4}\right]^{+}\right.$and $\left[\mathbf{L H}_{4}+\mathrm{CH}_{3} \mathrm{OH}\right]^{+}$marked with black asterisks) with the measured and the calculated isotopic pattern of $\mathbf{L H}_{2} \mathbf{F e}$ (grey bars). $[\mathrm{M}]^{+} \mathrm{m} / \mathrm{z}=1354.6002$ (calcd.: 1354.6010).

The resulting iron(III) complex $\mathbf{L} \mathbf{H}_{\mathbf{2}} \mathbf{F e C l}$ could be characterized via HRMS (Fig. 5.16). The synthesis of the corresponding Fe(II) complex was again not feasible and resulted in a mixture of the ferrous and ferric complex. Furthermore, the iron(II) complex was prone to decomposition more rapidly.

A more detailed spectroscopic characterization was hampered by remaining impurities 
and the presence of $\mathbf{L H}_{\mathbf{4}}$ and $\mathbf{L}\{\mathbf{F e C l}\}_{2}$. However, the monoiron complex was used for further complexation reactions to yield the corresponding heterobimetallic copper iron complex (Chap. 5.3).

It should be noted that synthesis of the monoiron complex was also possible with the use of $\mathrm{Fe}\left(\mathrm{BF}_{4}\right)_{2}$, but the resulting complex, most likely bearing a $\mathrm{BF}_{4}{ }^{-}$as counterion instead of a chloride as an axial ligand, was prone to faster decomposition than the chloride analog.

\subsection{Iron Copper Complex LCuFeCl}

With $\mathbf{L H}_{\mathbf{2}} \mathbf{F e C l}$ in hand, the synthesis of the iron copper complex $\mathbf{L C u F e C l}$ could be performed by addition of a copper(II) salt in a polar solvent. The resulting heterobimetallic complex incorporates two redox active metal ions, which in their reduced states are both potentially suitable for small molecule activation, like molecular oxygen. ${ }^{[59,162-164]}$ The crude reaction mixture of $\mathbf{L H}_{\mathbf{2}} \mathbf{F e C l}$ could be used for further complexation reactions (Fig. 5.6), even though remaining free base Siamese-twin porphyrin in the solution also led to the formation of the dicopper complex $\mathbf{L} \mathbf{C u}_{\mathbf{2}}$ upon addition of a copper salt.

\subsubsection{Synthesis of $\mathrm{LCuFeCl}$}

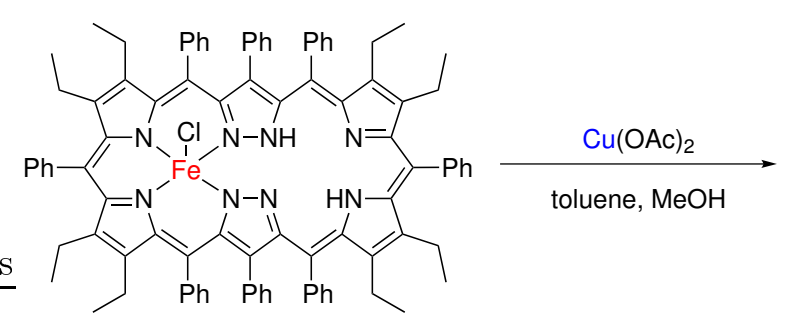

$\mathrm{LH}_{2} \mathrm{FeCl}$

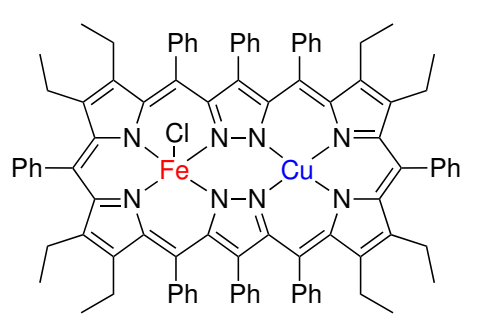

$\mathrm{LCuFeCl}$

Scheme 5.6: Synthesis of the heterobimetallic iron copper complex LCuFeCl.

The residue of the synthesis of $\mathbf{L H}_{\mathbf{2}} \mathbf{F e}$ was dissolved in toluene and added to a solution of copper acetate in $\mathrm{MeOH}$. The reaction mixture was stirred for $30 \mathrm{~min}$ at ambient temperature and the solvent was removed under reduced pressure. The residue was redissolved in $\mathrm{CH}_{2} \mathrm{Cl}_{2}$ and filtered over a plug of basic aluminum oxide to remove $\mathbf{L} \mathbf{C u}_{\mathbf{2}}$, which was the only fraction passing. The remaining $\mathbf{L C u F e C l}$ was eluted with $\mathrm{MeOH}$ and the solvent was removed under reduced pressure. The crude product was purified by column chromatography (silica, $n$-hexane/EtOAc 4:1) and recrystallized from a mixture of $\mathrm{CH}_{2} \mathrm{Cl}_{2}$ and $n$-hexane (1:1) to yield polycrystalline $\mathbf{L C u F e C l}$. No suitable single crystals for X-ray diffraction could be obtained so far. 


\subsubsection{Characterization of $\mathrm{LCuFeCl}$}

The crystallization behavior and electronic structure of the incorporated iron ion of $\mathbf{L C u F e C l}$ is assumed to be similar to that of $\mathbf{L N i F e C l}$, due to the similarity of $\mathrm{Cu}(\mathrm{II})$ and $\mathrm{Ni}(\mathrm{II})$ with regards to their coordination environment, as well as previously encountered similarities of the homobimetallic complexes $\mathbf{L} \mathbf{C u}_{\mathbf{2}}$ and $\mathbf{L} \mathbf{N i}_{\mathbf{2}}$.

The $\mathbf{L C u F e C l}$ could be characterized by HRMS, again as [M] ${ }^{+}$species (Fig. 5.17).

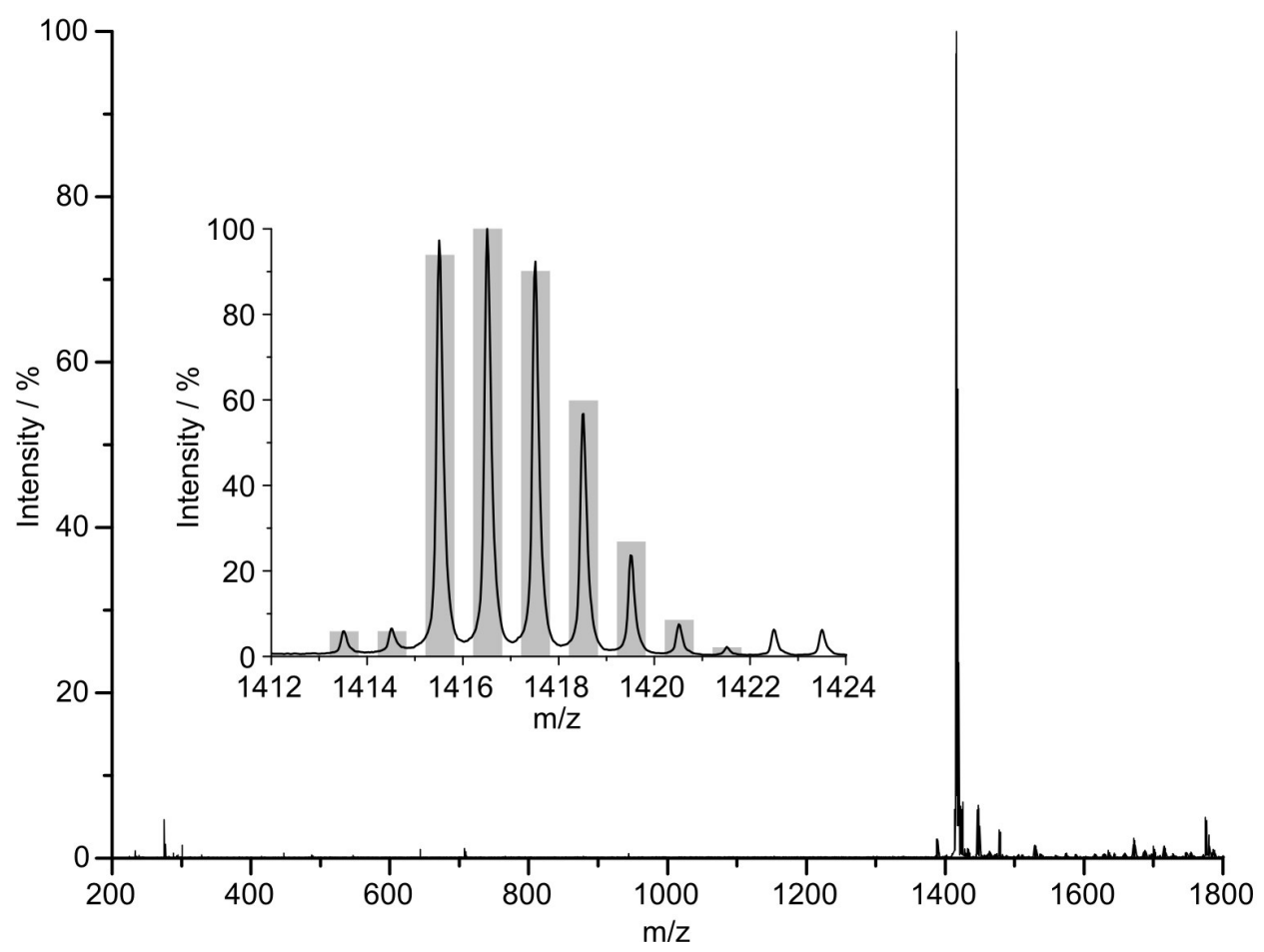

Figure 5.17: $\mathrm{HRMS} \mathrm{ESI}^{+}$spectrum of $\mathbf{L C u F e C l}$ in MeOH. Inset: Comparison of the measured (black) and the calculated isotopic pattern (grey bars). $[\mathrm{M}]^{+} \mathrm{m} / \mathrm{z}=1415.5149$ (calcd.: 1415.5150).

The overall optical spectrum of $\mathbf{L C u F e C l}$ looked similar to that of LNiFeCl (Fig. 5.18), even though all three absorption bands are shifted.

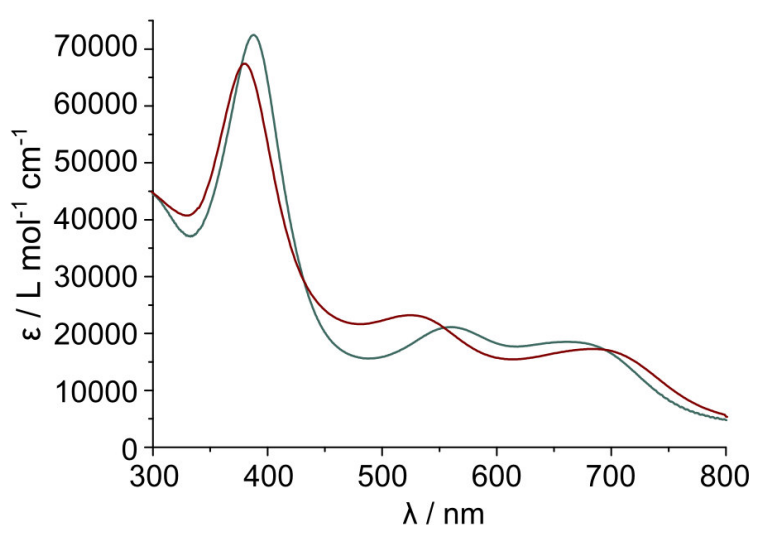

Figure 5.18: UV-vis spectra of $\mathbf{L C u F e C l}$ (blue) and $\mathbf{L N i F e C l}$ (brown) in $\mathrm{CH}_{2} \mathrm{Cl}_{2}$ at ambient temperature. 
Within the error of the measurement the red shifted Soret like band (388 nm) showed a similar extinction coefficient $(\epsilon=72500)$. The first Q-band appears at a wavelength of $560 \mathrm{~nm}$ and is red shifted, whereas the second Q-band (692 nm), which is assumed to have mainly some copper contribution, is slightly blue shifted.

EPR and Mößbauer spectroscopy as well as magnetic susceptibility measurements were performed on $\mathbf{L C u F e C l}$ to compare the iron ion's spin and oxidation state with those of LNiFeCl. Even though copper(II), as a $\mathrm{d}^{9}$ metal ion, has a spin of $\mathrm{S}=1 / 2$, no EPR signal could be obtained at temperatures above $120 \mathrm{~K}$. This is in contradiction to the previously synthesized copper complexes $\mathbf{L} \mathbf{C u}_{\mathbf{2}}$ and $\mathbf{L N i C u},{ }^{[53,55]}$ but can be explained with the presence of the iron(III) ion. Exchange coupling between the iron(III) and copper(II) metal ions can lead to an EPR silent spin ground state or can result in signal broadening too large to resolve a signal in X-band EPR spectroscopy at higher temperatures. Indeed, magnetic susceptibility measurements on solid material have been interpreted as an antiferromatically coupled two spin system with spins of $\mathrm{S}_{1}=3 / 2$ and $\mathrm{S}_{2}=1 / 2$ (Fig. 5.19).

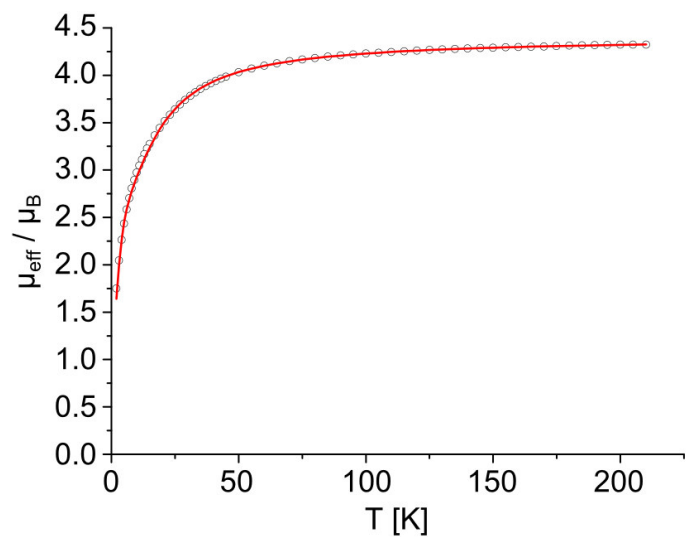

Figure 5.19: Susceptibility measurement of $\mathbf{L C u F e C l}$ (black circles) and its fit (red) with $\mathrm{g}_{1}=2.083, \mathrm{D}_{1}=-6.81, \mathrm{~g}_{2}=2.050$ and $\mathrm{J}=-6.40 \mathrm{~cm}^{-1}$.

An effective magnetic moment of $\mu_{e f f}=4.32$ at temperatures over $100 \mathrm{~K}$ is indicative of a spin system of $\mathrm{S}_{1}=3 / 2$ and $\mathrm{S}_{2}=1 / 2$, whose spin-only value would be 4.25 , assuming g-values of 2.0023. Two spin centers with $\mathrm{S}_{1}=3 / 2$ and $\mathrm{S}_{2}=1 / 2$ are in agreement with an is-iron(III) $(\mathrm{S}=3 / 2)$ and a copper(II) $(\mathrm{S}=1 / 2)$ ion. An intermediate spin iron(III) ion was expected from previous results for $\mathbf{L N i F e C l}$ and was further confirmed by Mößbauer spectroscopy (Fig. 5.20).

A doublet $\left(\delta_{F e}=0.55\right)$ with a quadrupol splitting of $\Delta E_{Q}=2.82$, which is typical for iron(III) intermediate spin, was observed in $80 \%$ area ratio together with a doublet with a distinct smaller quadrupol splitting $\left(\delta_{F e}=0.54, \Delta E_{Q}=1.36\right)$ in $20 \%$ area ratio. Both doublets are nearly identical with the doublets obtained for LNiFeCl (Table 5.6). Even though the synthesis of $\mathbf{L C u F e C l}$ and $\mathbf{L N i F e C l}$ was partly different, the ratio between the two characteristic Mößbauer doublets was retained, however. 


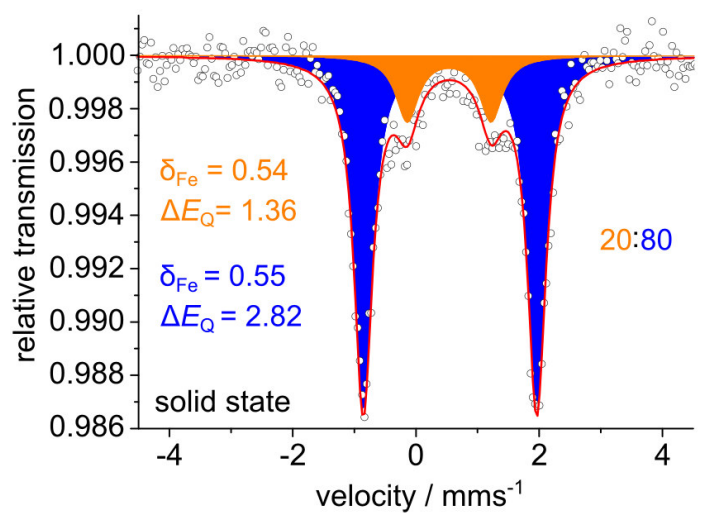

Figure 5.20: Solid state Mößbauer spectrum of $\mathbf{L C u F e C l}$ with $\delta_{F e}=0.54$ and 0.55 , $\Delta E_{Q}=1.36$ and 2.82 at $80 \mathrm{~K}$.

Table 5.6: Mößbauer parameters of $\mathbf{L C u F e C l}$ and $\mathbf{L N i F e C l}$ in solid state at $80 \mathrm{~K}$.

\begin{tabular}{c|c|c|c}
\hline Complex & $\delta_{F e}$ & $\Delta E_{Q}$ & rel. Int. /\% \\
\hline \multirow{2}{*}{ LCuFeCl } & 0.54 & 1.36 & 20 \\
& 0.55 & 2.82 & 80 \\
\hline \multirow{2}{*}{ LNiFeCl } & 0.53 & 1.20 & 20 \\
& 0.58 & 2.70 & 80 \\
\hline
\end{tabular}

Similar to $\mathbf{L N i F e C l}$, the redox chemistry of $\mathbf{L C u F e C l}$ was studied by cyclic and square wave voltammetry and showed the expected two reversible ligand-based oxidation events together with four irreversible reduction events (Fig. 5.21).

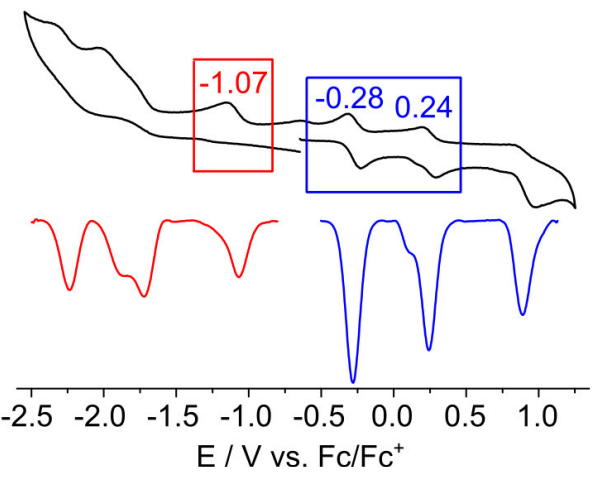

(a)

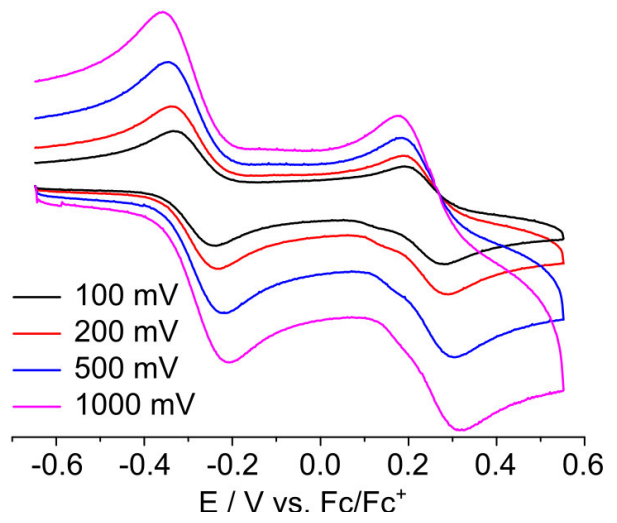

(b)

Figure 5.21: (a) CV (top) and SWV (bottom) curve of $\mathbf{L C u F e C l}$ at a scan rate of $100 \mathrm{mV}$. The two reversible ligand-based oxidations are marked in blue and the assumed iron(III) reduction in red. Half wave potentials $\left(\mathrm{E}_{1 / 2}\right)$ are indicated in volts and determined from square wave voltammetry. (b) CV curve of the electrochemically reversible oxidations of $\mathbf{L C u F e C l}$ at different scan rates indicated. Both measurements were performed in $\mathrm{CH}_{2} \mathrm{Cl}_{2}$ at ambient temperature with $0.1 \mathrm{M}\left[\mathrm{Bu}_{4} \mathrm{~N}\right] \mathrm{PF}_{6}$ as electrolyte referenced to the redox couple $\mathrm{Fc} / \mathrm{Fc}^{+}$.

The two reversible ligand-based oxidation waves at -0.28 and $+0.24 \mathrm{~V}$ vs. $\mathrm{Fc} / \mathrm{Fc}^{+}$and the reduction events at -1.07 and -1.71 or $-1.85 \mathrm{~V}$ are comparable with the measured 
values for the other metal complexes of the Siamese-twin porphyrin (Tab. 5.7). The two reduction waves at -1.71 and $1.85 \mathrm{~V}$ interfere and can not be explained.

Table 5.7: Potentials of the first reduction and the first two oxidations of $\mathbf{L C u F e C l}^{[\mathrm{a}]}$ in comparison to the iron, nickel and copper complexes of the STP vs. Fc $/ \mathrm{Fc}^{+}$.

\begin{tabular}{l|c|c|c|c|c}
\hline Complex & $\begin{array}{c}E \text { Red / V } \\
\text { ligand }\end{array}$ & $\begin{array}{c}E \text { Red / V } \\
\text { metal }\end{array}$ & $E$ Ox 1/V & $E$ Ox 2/V & Ref. \\
\hline $\mathbf{L C u F e C l}$ & $-1.71 /-1.85^{[\mathrm{b}]}$ & $-1.07^{[\mathrm{b}]}$ & -0.28 & 0.24 & - \\
$\mathbf{L N i F e C l}$ & -1.92 & $-1.12^{[\mathrm{b}]}$ & -0.08 & 0.33 & - \\
$\mathbf{L N i}{ }_{2}{ }^{[c]}$ & -1.90 & - & -0.16 & 0.30 & {$[55]$} \\
$\mathbf{L C \mathbf { C u } ^ { i }}$ & $-1.81^{[\mathrm{b}]}$ & - & -0.34 & 0.24 & {$[55]$} \\
$\mathbf{L C u}_{\mathbf{2}}{ }^{[\mathrm{c}]}$ & -1.86 & - & -0.37 & -0.02 & {$[55]$} \\
\hline
\end{tabular}

[a] Values as determined by square wave voltammetry (in $\mathrm{CH}_{2} \mathrm{Cl}_{2}, 0.1 \mathrm{M}\left[\mathrm{Bu}_{4} \mathrm{~N}\right] \mathrm{PF}_{6}$ ) cf. to Fig. 5.8. [b] Electrochemically irreversible. [c] The values reported previously ${ }^{[55]}$ have been incorrectly referenced because of conflicting data in literature. ${ }^{[141]} \mathrm{A}$ potential of $-0.48 \mathrm{~V}$ of decamethylferrocene vs. ferrocene was choosen instead of -0.59 . A correction of $-0.11 \mathrm{~V}$ was done in this table.

The first oxidation of the STP scaffold of $\mathbf{L C u F e C l}$ occurs at a distinct lower potential $(-0.34 \mathrm{~V})$ than the first oxidation in $\mathbf{L N i F e C l}(-0.08 \mathrm{~V})$ but is in the same region as the first oxidation of the copper complexes $\mathbf{L C u N i}$ and $\mathbf{L} \mathbf{C} \mathbf{u}_{2}$. This indicates, that the first oxidation of $\mathbf{L C u F e C l}$ takes place at the dipyrromethene unit at the copper side. The iron-based reduction wave at $-1.07 \mathrm{~V}$ vs. $\mathrm{Fc} / \mathrm{Fc}^{+}$is within the same region as it is for $\mathbf{L N i F e C l}\left(-1.12 \mathrm{~V}\right.$ vs. $\left.\mathrm{Fc} / \mathrm{Fc}^{+}\right)$and should therefore also be accessible for reduction with $\mathrm{CoCp}_{2}$, to yield the ferrous complex LCuFe. However, further investigations have yet to be done to analyze the redox chemistry of $\mathbf{L C u F e C l}$ in more detail. Additionally, other heterobimetallic complexes can be synthesized starting from $\mathbf{L H}_{\mathbf{2}} \mathbf{F e C l}$.

\subsection{Diiron Complex $\mathrm{L}\{\mathrm{FeCl}\}_{2}$}

After the characterization and investigation of the redox behavior of $\mathbf{L N i F e C l}$ the corresponding homobimetallic complex $\mathbf{L}\{\mathbf{F e C l}\}_{2}$ was synthesized. As expected, the incorporation of two iron(III) ions led to a complication of the spectroscopic data.

\subsubsection{Synthesis of $\mathrm{L}\{\mathrm{FeCl}\}_{2}$}

According to the procedure developed for $\mathbf{L N i F e C l , ~ t h e ~ c o r r e s p o n d i n g ~ h o m o b i m e t a l l i c ~}$ complex $\mathbf{L}\{\mathbf{F e C l}\}_{\mathbf{2}}$ was synthesized from $\mathbf{L} \mathbf{H}_{\mathbf{4}}$ with the use of $\mathrm{FeCl}_{2}$ and $\mathrm{NaOAc}$ (Fig. 5.7). Strikingly $\mathbf{L}\{\mathbf{F e C l}\}_{2}$ was found to be more sensitive than $\mathbf{L N i F e C l}$. Column chromatography always led to decomposition of the complex, no matter which solid phase was used. Only with size exclusion chromatography $\mathbf{L}\{\mathbf{F e C l}\}_{2}$ could be separated. 


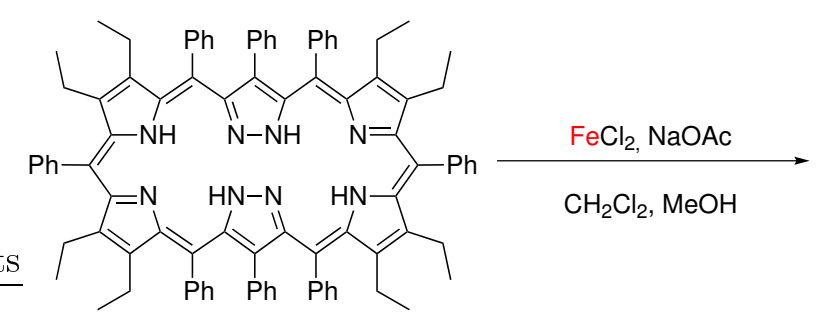

$\mathrm{LH}_{4}$

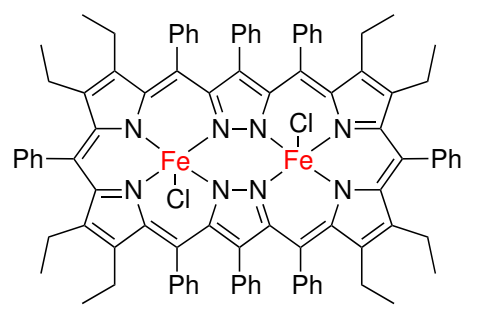

$\mathrm{L}\{\mathrm{FeCl}\}_{2}$

Scheme 5.7: Synthesis of the diiron complex $\mathrm{L}\{\mathrm{FeCl}\}_{2}$.

$\mathrm{FeCl}_{2}$ and $\mathrm{NaOAc}$ were added to a solution of $\mathbf{L H}_{\mathbf{4}}$ in a polar solvent and the reaction mixture was stirred for 30 minutes at ambient temperature. The solvent was removed under reduced pressure, $\mathrm{Et}_{2} \mathrm{O}$ was added and the suspension was filtered. The solvent was removed again and the residue was redissolved in $\mathrm{CH}_{2} \mathrm{Cl}_{2}$, washed with brine and dried over sodium sulfate. The raw product was purified by size exclusion chromatography and recrystallized from $n$-heptane and chlorobenzene.

\subsubsection{Characterization of $\mathrm{L}\{\mathrm{FeCl}\}_{2}$}

$\mathbf{L}\{\mathbf{F e C l}\}_{\mathbf{2}}$ could be detected in HRMS (MeOH solution) as $\left[\mathrm{M}+\mathrm{CH}_{3} \mathrm{O}\right]^{+}$and $[\mathrm{M}]^{2+}$ (Fig. 5.22). Due to its two iron(III) ions the corresponding complex $\mathbf{L F e F e} \mathbf{2}^{\mathbf{2}+}$ is twice positively charged after decoordination of the axial chloride ligands; loss of the chloride ligand unter ESI-MS conditions was also observed for LNiFeCl. One of the positive charges is compensated with a methanolate which is present because the measurements were performed in $\mathrm{MeOH}$, resulting in the formation of $\left[\mathrm{M}+\mathrm{CH}_{3} \mathrm{O}\right]^{+}$ions.

The molecular structure of $\mathbf{L}\{\mathbf{F e C l}\}_{\mathbf{2}}$, as determined by single crystal X-ray diffraction, confirmed the assumed double oxidation of $\mathbf{L F e F e}$, due to an axial coordinated chloride ligand for each iron ion, which most likely occurs in a ferric iron complex, resulting in an overall neutral compound (Fig. 5.23) as in LNiFeCl (cf. Fig. 5.2).

Due to the twist of the Siamese-twin porphyrin, one side of each coordination pocket is more shielded than the other one, because of the orientation of the phenyl and ethyl groups. Thus, the coordination of the axial chloride ligand always occurs from the less shielded site, resulting in a trans-coordination of the chlorides (Fig. 5.23 (b)). This trans-configuration has to be taken into account in future reactivity studies of bimetallic complexes of the STP towards small molecule activation and transfer to a substrate. 


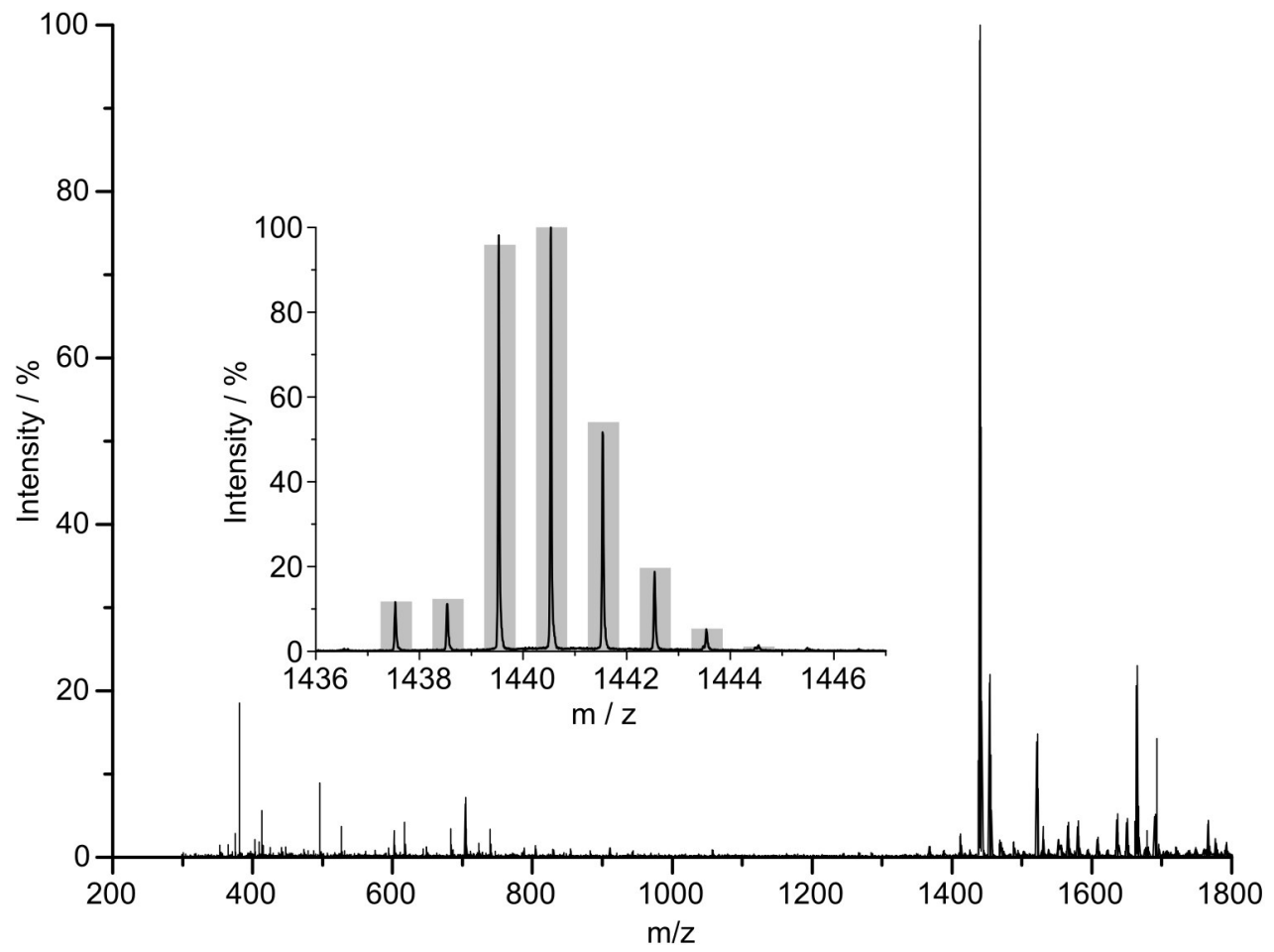

Figure 5.22: HRMS ESI ${ }^{+}$spectrum of crystalline $\mathbf{L}\{\mathbf{F e C l}\}_{2}$ in $\mathrm{MeOH}$. Inset: Comparison of the measured (black) and calculated isotopic pattern (grey bars). $\left[\mathrm{M}+\mathrm{CH}_{3} \mathrm{O}\right]^{+}$ $\mathrm{m} / \mathrm{z}=1439.5375$ (calcd.: 1439.5386).

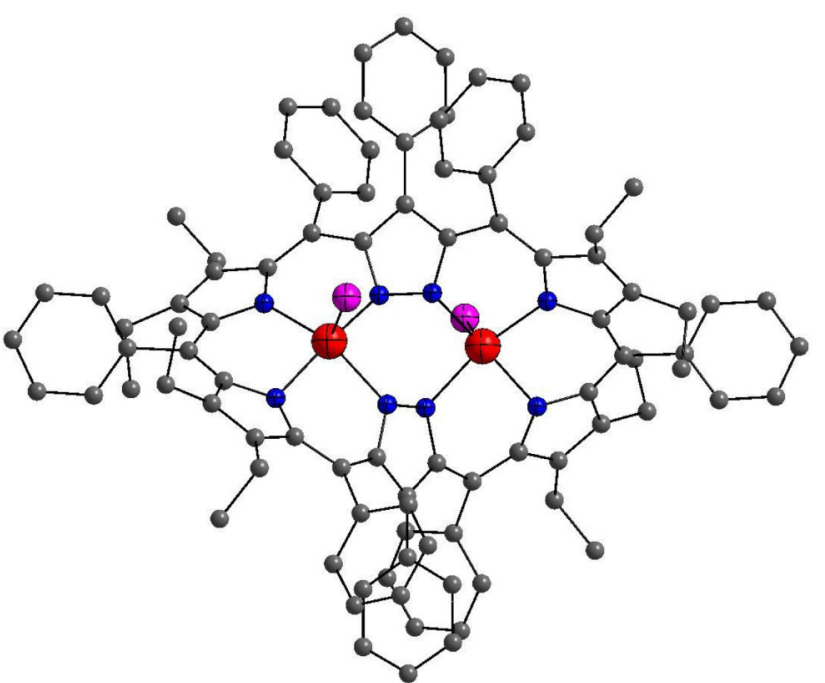

(a)

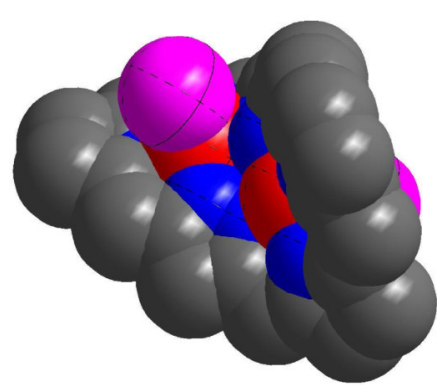

(b)

Figure 5.23: (a) Ball-and-stick model of the molecular structure of $\mathrm{L}\{\mathrm{FeCl}\}_{2}$ and (b) stick model of the sideview of the core-structure, without any substituent, of $\mathbf{L}\{\mathbf{F e C l}\}_{2}$, approximately along the iron nickel axis, determined by single crystal X-ray diffraction. All hydrogen atoms and solvent molecules were omitted for clarity (grey: carbon, blue: nitrogen, red: iron, pink: chlorine) For details, see Appendix. 
$\mathbf{L}\{\mathbf{F e C l}\}_{2}$ showed the expected absorption spectrum with an extintion coefficient of 67400 for the Soret like band (382 nm) like in LNiFeCl $(\lambda=380 \mathrm{~nm}, \epsilon=67400)$ and LCuFeCl $(\lambda=388 \mathrm{~nm}, \epsilon=72500)$.

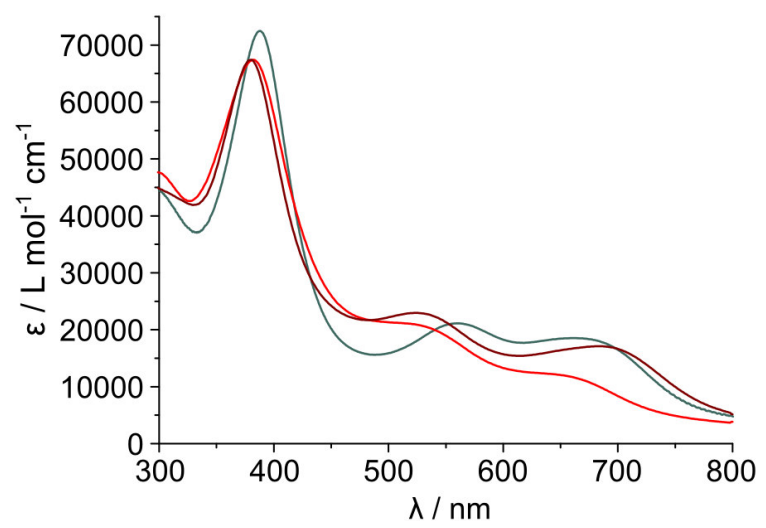

Figure 5.24: UV-vis spectra of $\mathrm{L}\{\mathbf{F e C l}\}_{2}$ (red), $\mathbf{L N i F e C l}$ (brown) and $\mathbf{L C u F e C l}$ (blue) in $\mathrm{CH}_{2} \mathrm{Cl}_{2}$ at ambient temperature.

The first Q-band appears at the same wavelength of $535 \mathrm{~nm}(\epsilon=20400)$ as in LNiFeCl $(\epsilon=22700)$, further indicating that this absorption band has some iron contribution. The second Q-band at $667 \mathrm{~nm}$ is significantly weaker $(\epsilon=11300)$ and blue shifted with regards to $\mathbf{L N i F e C l}(\lambda=700 \mathrm{~nm}, \epsilon=16600)$ and $\mathbf{L C u F e C l}$ $(\lambda=692 \mathrm{~nm}, \epsilon=17300$ ) (Fig. 5.24), further indicating that this absorption band has mainly some nickel and copper contribution in $\mathbf{L N i F e C l}$ and $\mathbf{L C u F e C l}$, respectively.

For further insight into the electronic structure of the diiron complex, EPR, Mößbauer and magnetic susceptibility measurements were performed. In this case the diiron complex showed no signal in the X-band EPR spectrum, due to typical signal broadening in a dinuclear complex having two spin centers with a spin of $S>1 / 2$ each, because of exchange coupling. ${ }^{[2,165]}$ The spin state of the iron(III) ion could therefore only be determined by magnetic susceptibility measurements (Fig. 5.25) and Mößbauer spectroscopy (Fig. 5.26).

An effective magnetic moment of $\mu_{e f f}=5.92$, as determined by magnetic susceptibility measurements (Fig. 5.25), indicated two $\mathrm{S}=3 / 2$ spins, whose spin-only value according to equation (2) would be $\mu_{\text {eff }}=5.48$. A spin system of two times $\mathrm{S}=3 / 2$ means that both iron ions are in the intermediate spin state, like observed for the iron(III) ion in LNiFeCl (Chap. 5.1.2) and $\mathbf{L C u F e C l ( C h a p . ~ 5 . 3 . 2 ) . ~ A ~ v e r y ~ s m a l l ~ a n t i f e r r o - ~}$ magnetic coupling with $J=-0.17 \mathrm{~cm}^{-1}$ was observed for $\mathbf{L}\{\mathbf{F e C l}\}_{2}$ like in $\mathbf{L C u F e C l}$ $\left(J=-6.40 \mathrm{~cm}^{-1}\right)$ whereas the previously synthesized dicopper complex $\mathbf{L} \mathbf{C u}_{\mathbf{2}}$ showed ferromagnetic coupling due to orthogonal magnetic orbitals. ${ }^{[53]}$ 


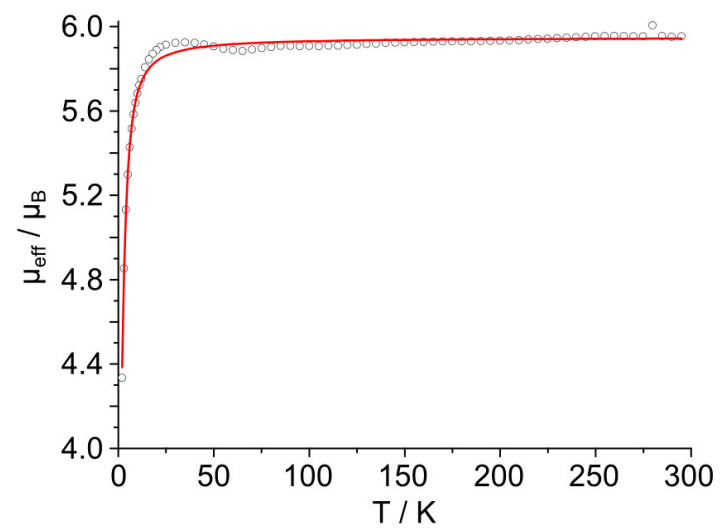

Figure 5.25: Susceptibility measurement of $\mathrm{L}\{\mathrm{FeCl}\}_{2}$ (black circles) and its fit (red) with $\mathrm{g}=2.172, \mathrm{D}=3.01, \mathrm{E} / \mathrm{D}=0$ and a slight antiferromagnetical coupling of $J=-0.17$.

To determine the iron ion's oxidation and spin state in more detail, Mößbauer (MB) spectroscopy was performed on solid samples of $\mathbf{L}\{\mathbf{F e C l}\}_{\mathbf{2}}$ (Fig. 5.26) and compared to the results for $\mathbf{L N i F e C l}$ and $\mathbf{L C u F e C l}$ (Tab. 5.6).

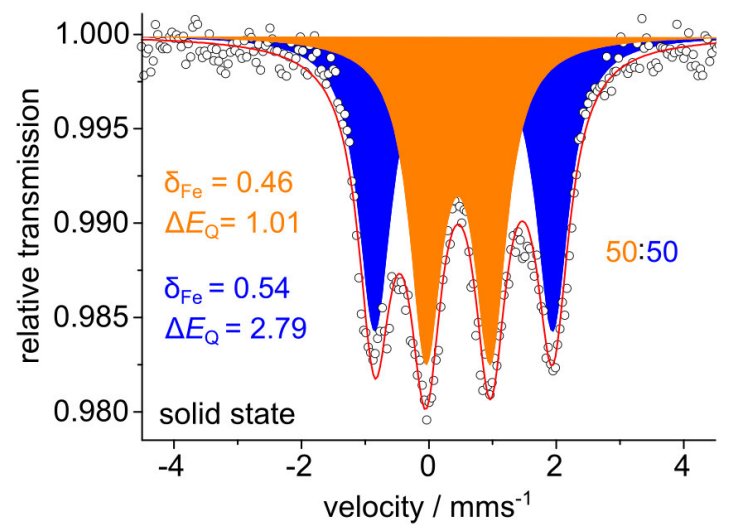

Figure 5.26: Solid state Mößbauer spectrum of $\mathbf{L}\{\mathbf{F e C l}\}_{2}$ with $\delta_{F e}=0.46$ and 0.54 and $\Delta E_{Q}=1.01$ and 2.79 at $80 \mathrm{~K}$.

The Mößbauer spectrum of $\mathbf{L}\{\mathbf{F e C l}\}_{2}$ showed two different doublets in a ratio of 50:50, one with a large quadrupole splitting of $\Delta E_{Q}=2.79$ and one with a smaller splitting of $\Delta E_{Q}=1.01$. The isomer shift and the quadrupol splitting of both doublets are in good agreement with the doublets observed in $\mathbf{L N i F e C l}$ and $\mathbf{L C u F e C l}$ (Tab. 5.6).

For $\mathbf{L}\{\mathbf{F e C l}\}_{\mathbf{2}}$ the ratio of the two doublets is 50:50 while it is 20:80 in the case of $\mathbf{L N i F e C l}$ and $\mathbf{L C u F e C l}$. On the first glance, it seemed that both iron ions in $\mathbf{L}\{\mathbf{F e C l}\}_{\mathbf{2}}$ are different, due to two different Mößbauer doublets in a ratio of 1:1. However, $\mathbf{L}\{\mathbf{F e C l}\}_{2}$ 's solid state structure showed that both Fe(III) ions are identical from a crystallographic point of view. Furthermore, $\mathbf{L N i F e C l}$ and $\mathbf{L C u F e C l}$ have also shown two different doublets. 
Table 5.8: Mößbauer parameter of $\mathrm{L}\{\mathrm{FeCl}\}_{2}, \mathbf{L C u F e C l}$, and $\mathbf{L N i F e C l}$ in the solid state at $80 \mathrm{~K}$.

\begin{tabular}{l|c|c}
\hline Complex & $\delta_{F e}$ & $\Delta E_{Q}$ \\
\hline $\multirow{2}{*}{\mathbf{F e C l}}_{\mathbf{2}}$ & 0.46 & 1.01 \\
& 0.54 & 2.79 \\
\hline \multirow{2}{*}{$\mathbf{L N i F e C l}$} & 0.53 & 1.20 \\
& 0.58 & 2.70 \\
\hline \multirow{2}{*}{$\mathbf{L C u F e C l}$} & 0.54 & 1.36 \\
& 0.55 & 2.82 \\
\hline
\end{tabular}

The redox chemistry of $\mathbf{L}\{\mathbf{F e C l}\}_{2}$ was studied by cyclic and square wave voltammetry and showed the expected two ligand based oxidation events.

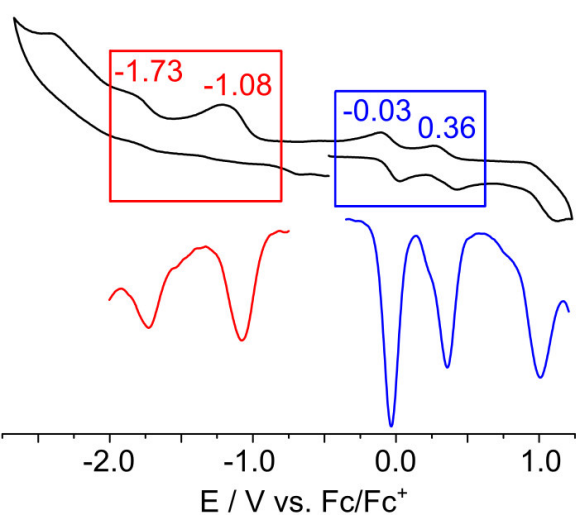

(a)

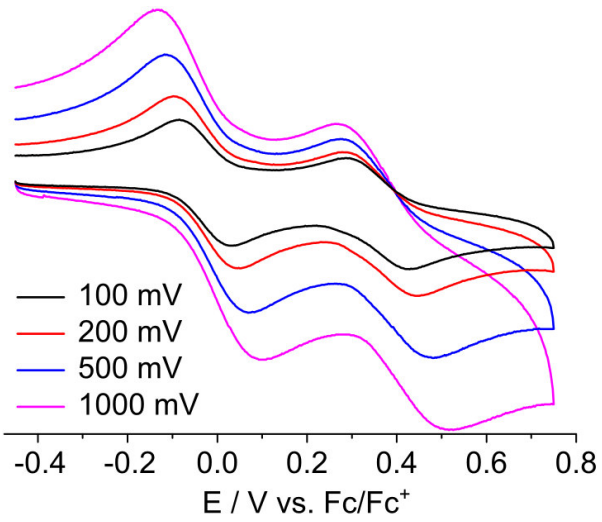

(b)

Figure 5.27: (a) CV (top) and SWV (bottom) curve of $\mathbf{L}\{\mathbf{F e C l}\}_{2}$ in $\mathrm{CH}_{2} \mathrm{Cl}_{2}$ at ambient temperature with $\left[\mathrm{Bu}_{4} \mathrm{~N}\right] \mathrm{PF}_{6}$ as electrolyte at a scan rate of $100 \mathrm{mV}$ referenced to the redox couple $\mathrm{Fc} / \mathrm{Fc}^{+}$. The two ligand-based oxidations are marked in blue and the two presumptive iron reduction waves in red. Half wave potentials $\left(\mathrm{E}_{1 / 2}\right)$ are indicated in volts and determined from square wave voltammetry. (b) CV curve of the electrochemically (quasi)reversible oxidations of $\mathbf{L}\{\mathbf{F e C l}\}_{2}$ at different scan rates indicated. Measurements were performed in $\mathrm{CH}_{2} \mathrm{Cl}_{2}$ at ambient temperature with $0.1 \mathrm{M}\left[\mathrm{Bu}_{4} \mathrm{~N}\right] \mathrm{PF}_{6}$ as electrolyte referred to the redox couple $\mathrm{Fc} / \mathrm{Fc}^{+}$.

The first of the two ligand based oxidation waves at $-0.03 \mathrm{~V}$ is reversible whereas the second one and $+0.36 \mathrm{~V}$ vs. $\mathrm{Fc} / \mathrm{Fc}^{+}$becomes quasireversible for $\mathbf{L}\{\mathbf{F e C l}\}_{\mathbf{2}}$, further indicating its lower stability. Additionaly the first reduction wave at $-1.08 \mathrm{~V}$ is within the same region as for $\mathbf{L N i F e C l}(-1.12 \mathrm{~V})$ and $\mathbf{L C u F e C l}(-1.08 \mathrm{~V})$ (Tab. 5.9) and a second reduction wave at $-1.73 \mathrm{~V}$ vs $\mathrm{Fc} / \mathrm{Fc}^{+}$appears. Two metal-based reductions are theoretically possible, due to the presence of two iron(III) ions. It has to be mentioned, that the SWV spectra result from two measurements, one for the oxidation and one for the reduction. Therefore comparison of the integrals of the redox peaks of the oxidation and the reduction is not suitable to obtain the number of transfered electrons during the reduction events. 
Table 5.9: Potentials of the first reduction and the first two oxidations of $\mathbf{L}\{\mathbf{F e C l}\}_{2}{ }^{[\mathrm{a}]}$ in comparison to the iron, nickel and copper complexes of the STP vs. $\mathrm{Fc} / \mathrm{Fc}^{+}$.

\begin{tabular}{l|c|c|c|c|c}
\hline Complex & $\begin{array}{c}E \text { Red } / \mathrm{V} \\
\text { ligand }\end{array}$ & $\begin{array}{c}E \text { Red / V } \\
\text { metal }\end{array}$ & $E$ Ox 1/V & $E$ Ox 2/V & Ref. \\
\hline $\mathbf{L}\{\mathbf{F e C l}\}_{\mathbf{2}}$ & -2.27 & $-1.73^{[\mathrm{b}]}$ and $-1.08^{[\mathrm{b}]}$ & -0.03 & 0.36 & - \\
$\mathbf{L N i F e C l}$ & -1.92 & $-1.12^{[\mathrm{b}]}$ & -0.08 & 0.33 & - \\
$\mathbf{L C u F e C l}$ & $-1.71 /-1.85^{[\mathrm{b}]}$ & $-1.07^{[\mathrm{b}]}$ & -0.28 & 0.24 & - \\
$\mathbf{L} \mathbf{N i}_{\mathbf{2}}[\mathrm{c}]$ & -1.90 & - & -0.16 & 0.30 & {$[55]$} \\
$\mathbf{L C u N i}[\mathrm{c}]$ & $-1.81^{[\mathrm{b}]}$ & - & -0.34 & 0.24 & {$[55]$} \\
$\mathbf{L C u}_{\mathbf{2}}{ }^{[\mathrm{c}]}$ & -1.86 & - & -0.37 & -0.02 & {$[55]$} \\
\hline
\end{tabular}

[a] Values as determined by square wave voltammetry (in $\mathrm{CH}_{2} \mathrm{Cl}_{2}, 0.1 \mathrm{M}\left[\mathrm{Bu}_{4} \mathrm{~N}\right] \mathrm{PF}_{6}$ ) cf. to Fig. 5.8. [b] Electrochemically irreversible. [c] The values reported previously ${ }^{[55]}$ have been incorrectly referenced because of conflicting data in literature. ${ }^{[141]} \mathrm{A}$ potential of $-0.48 \mathrm{~V}$ of decamethylferrocene vs. ferrocene was choosen instead of -0.59 . A correction of $-0.11 \mathrm{~V}$ was done in this table.

The (quasi)reversible oxidation events for $\mathbf{L}\{\mathbf{F e C l}\}_{\mathbf{2}}$ occured at similiar potentials as for LNiFeCl and are only sligthly shifted to higher potentials (Fig. 5.9). The oxidation locus for both oxidations of $\mathbf{L N i F e C l}$ was therefore difficult to determine.

Furthermore, $\mathbf{L}\{\mathbf{F e C l}\}_{2}$ showed decomposition during $\mathrm{CV}$ measurements at potentials higher than $1.2 \mathrm{~V}$ and lower than $-1.3 \mathrm{~V}$ vs. $\mathrm{Fc} / \mathrm{Fc}^{+}$, as also indicated by instant UV-vis spectroscopy.

It has been shown for $\mathbf{L N i F e C l}$ that single oxidation led to an unstable compound and $\mathbf{L}\{\mathbf{F e C l}\}_{2}$ is formally already oxidized once more due to the presence of a second iron(III) ion. With an additional oxidation, decomposition is expected to be much faster, not to mention a second oxidation.

The instability of $\mathbf{L}\{\mathbf{F e C l}\}_{2}$ also affected its reduction. Reduction with $\mathrm{CoCp}_{2}$ like for $\mathbf{L N i F e C l}$ always led to decomposition, even at low temperatures.

Nevertheless, when the redox chemistry of the STP's iron complexes is understood in more detail, $\mathbf{L}\{\mathbf{F e C l}\}_{\mathbf{2}}$ can be a suitable precursor complex for reactivity studies towards small molecule activation. 


\section{Cobalt Complexes of the Siamese-Twin Porphyrin}

Since cobalt(II) is a redox active $\mathrm{d}^{7}$ metal ion, it is interesting for catalysis and oxygen activation ${ }^{[104,166-168]}$ and would be the missing link between the known $\mathrm{d}^{5}-\mathrm{d}^{6}\left(\mathrm{Fe}^{\mathrm{III}}, \mathrm{Fe}^{\mathrm{II}}\right)$ and $\mathrm{d}^{8}-\mathrm{d}^{9}\left(\mathrm{Ni}^{\mathrm{II}}, \mathrm{Cu}^{\mathrm{II}}\right)$ metal complexes of the Siamese-twin porphyrin. ${ }^{[10,53-55,95]}$

\subsection{Nickel Cobalt Complex LNiCo}

Initially, the nickel(II) cobalt(II) complex LNiCo has been synthesized. Like LNiFeCl and LNiFe (Chap. 5) the presence of only one cobalt ion in LNiCo should simplify the cobalt ion's characterization.

\subsubsection{Synthesis of LNiCo}

Interestingly the nickel cobalt complex could not be synthesized according to $\mathbf{L N i C u}{ }^{[55]}$ using the corresponding cobalt acetate $\mathrm{Co}(\mathrm{OAc})_{2}$. In contrast to $\mathbf{L N i F e C l}$ a complexation with the corresponding cobalt chloride salt $\mathrm{CoCl}_{2}$ did not led to a complexation of cobalt. Also the addition of pyridine as an axial ligand, due to the cobalt ion's tendency to adopt a five or six fold coordination and its affinity towards additional nitrogen based ligands, ${ }^{8]}$ did not yield the cobalt complex of the Siamese-twin porphyrin. Only the use of $\mathrm{Co}\left(\mathrm{BF}_{4}\right)_{2}$ under inert conditions succesfully generated LNiCo.

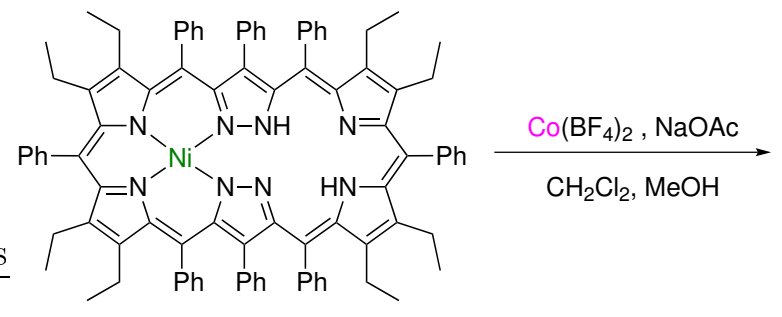

$\mathrm{LH}_{2} \mathrm{Ni}$

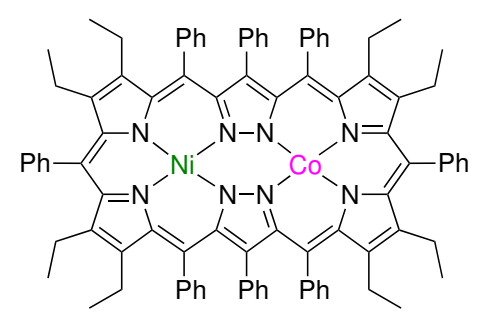

LNiCo

Scheme 6.1: Synthesis of the heterobimetallic complex LNiCo. 
$\mathrm{Co}\left(\mathrm{BF}_{4}\right)_{2}$ and $\mathrm{NaOAc}$ were added to a solution of the mononickel complex $\mathbf{L H}_{\mathbf{2}} \mathbf{N i}$ in polar solvent under inert conditions. The reaction mixture was stirred at ambient temperature for 30 minutes. The solvent was removed under reduced pressure and the residue was filtered over a plug of basic aluminum oxide, yielding LNiCo .

\subsubsection{Characterization of LNiCo}

Like all other metal complexes of the STP, LNiCo could be observed in mass spectrometry as $[\mathrm{M}]^{+}$species (Fig. 6.1) and could be characterized by HRMS.

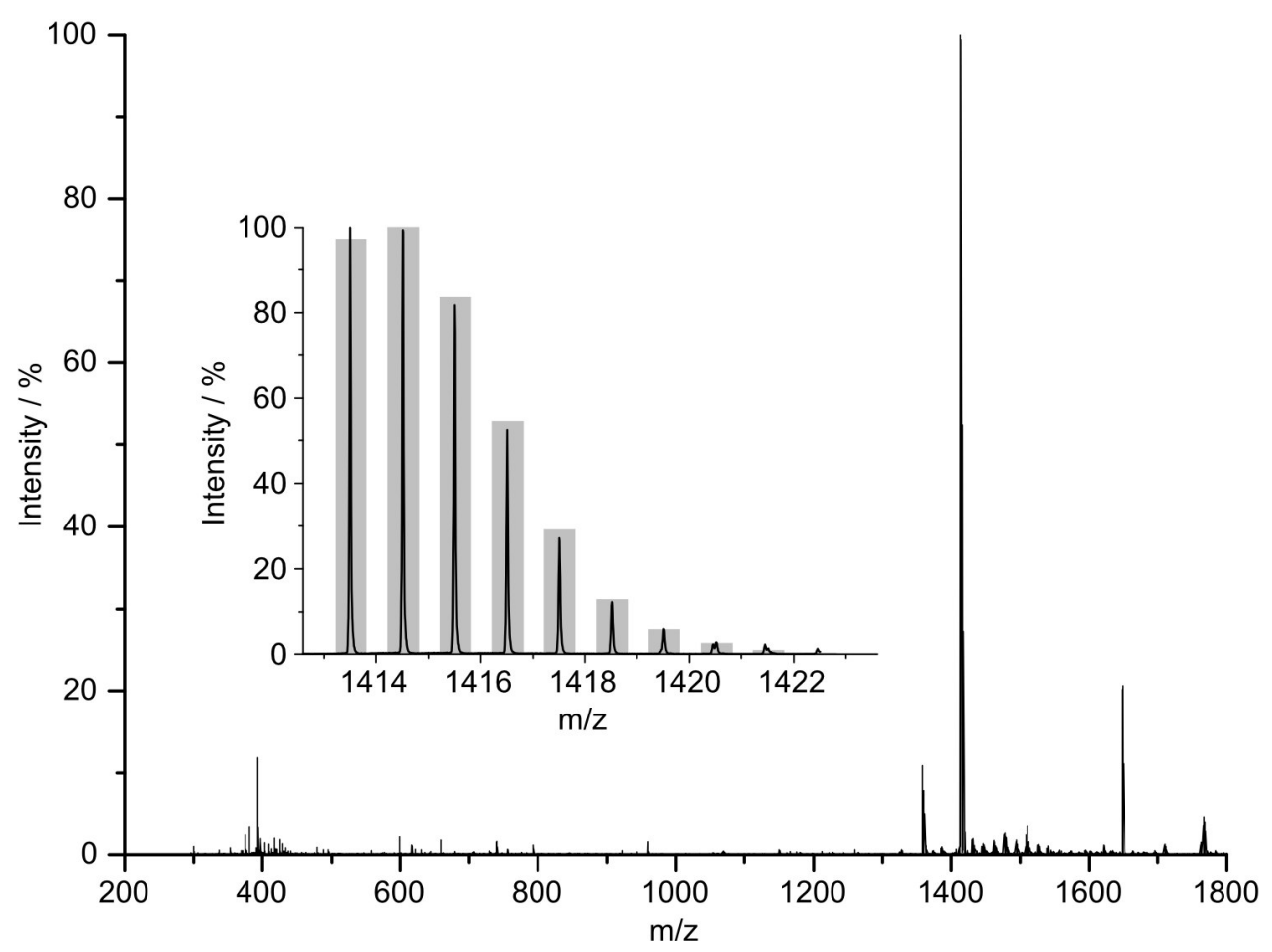

Figure 6.1: $\mathrm{HRMS} \mathrm{ESI}^{+}$spectrum of $\mathbf{L N i C o}$ in $\mathrm{MeOH}$. Inset: Comparison of the measured (black) and calculated isotopic pattern (grey bars). $[\mathrm{M}]^{+} \mathrm{m} / \mathrm{z}=1413.5186$ (calcd.: 1413.5171).

Interestingly LNiCo showed rapid decomposition, as determined by mass spectrometry, in solution as well as in solid state, even if it was stored under inert conditions in the freezer. This decomposition could also be observed in UV-vis spectroscopy. Hardly any STP like spectra could be observed, even if the solution of LNiCo was freshly prepared and measured immediately under inert condition.

EPR spectroscopic investigation indicated an organic radical $\left(\mathrm{g}_{x}=1.9643, \mathrm{~g}_{y}=1.9892\right.$, $\left.\mathrm{g}_{z}=2.0060\right)$ along with an unpaired electron located at the cobalt $\left(\mathrm{g}_{x}=2.1050\right.$, $\mathrm{g}_{y}=2.2853, \mathrm{~g}_{z}=2.3054$ ) (Fig. 6.2 black), suggesting an oxidized ligand together with a low-spin cobalt(II) $(\mathrm{S}=1 / 2)$ metal ion. ${ }^{[169-171]}$ With the addition of $\mathrm{Bu}_{4} \mathrm{NCl}$, intro- 
ducing a chloride as a medium strong ligand, both EPR signals nearly vanished (Fig. 6.2 red) indicating coordination of the chloride in the cobalt ion's axial position.

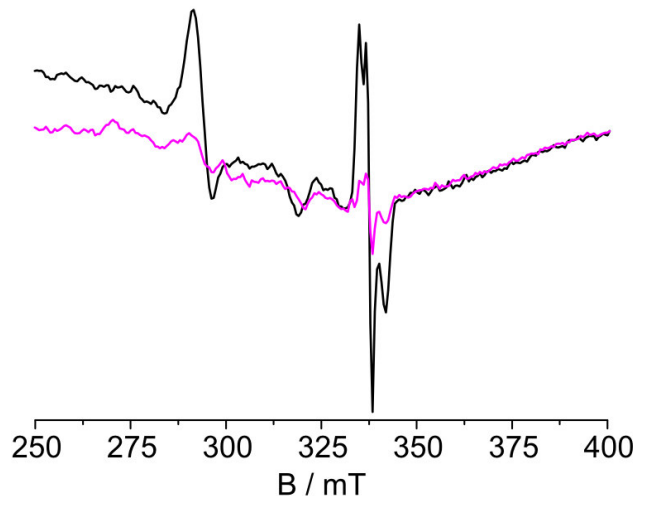

(a)

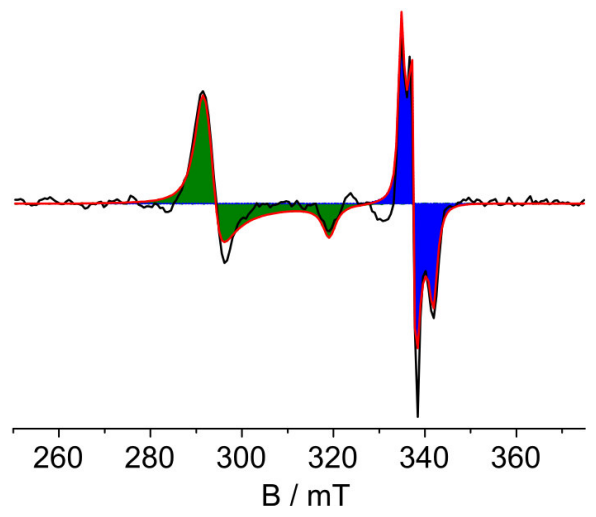

(b)

Figure 6.2: (a) EPR spectra of $\mathbf{L N i C o}$ in $\mathrm{CH}_{2} \mathrm{Cl}_{2}$ at $152 \mathrm{~K}$ before (black) and after (pink) addition of $\mathrm{Bu}_{4} \mathrm{NCl}$. (b) Simulated EPR spectrum (red) of LNiCo (black) with $\mathrm{g}_{x}=1.9643, \mathrm{~g}_{y}=1.9892, \mathrm{~g}_{z}=2.0060$ (Gaussian line shape, line width: $\mathrm{W}_{x}=24 \mathrm{G}$, $\mathrm{W}_{y}=10 \mathrm{G}, \mathrm{W}_{z}=16 \mathrm{G}$ ) for the organic radical (blue) and $\mathrm{g}_{x}=2.1050, \mathrm{~g}_{y}=2.2853$, $\mathrm{g}_{z}=2.3054$ (Gaussian line shape, line width: $\mathrm{W}_{x}=50 \mathrm{G}, \mathrm{W}_{y}=50 \mathrm{G}, \mathrm{W}_{z}=40 \mathrm{G}$ ) for the cobalt(II) species (green) with an amplitude ratio of 1:4.

Upon coordination of the chloride ligand the oxidation locus of $\mathbf{L N i C o}^{+}$is shifted from the porphyrin scaffold to the cobalt(II) ion (Scheme 6.2), resulting in a switch from $\mathrm{d}^{7}$ to $\mathrm{d}^{6}$ configuration of the cobalt ion. A cobalt(III) ion is stabilized by the coordination of the chloride ligand and the accrued diamagnetic low spin cobalt(III) ion ( $\mathrm{S}=0$ ) give rise to an EPR silent complex. ${ }^{[172]}$ These findings support the assumption of a formerly ligand-centered oxidation locus in $\mathbf{L N i C o}^{+}$.

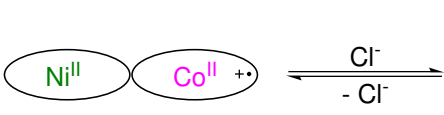

$\mathrm{LNiCo}^{+}$

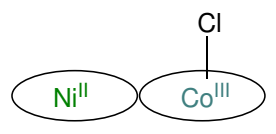

LNiCoCl

Scheme 6.2: Schematic representation of the shift of the oxidation locus in $\mathrm{LNiCo}^{+}$upon addition of chloride.

A coexistance of a ligand and a metal-based oxidation has been observed previously by BLusch for the oxidized nickel complexes $\mathbf{L H}_{\mathbf{2}} \mathbf{N i}, \mathbf{L N i} \mathbf{2}_{\mathbf{2}}$ and $\mathbf{L N i C u}$, whereat the ligand based oxidation has always represented the major species. ${ }^{[55,86]}$ If non-distilled $\mathrm{CH}_{2} \mathrm{Cl}_{2}$ is used for EPR measurements of $\mathbf{L N i}_{2}{ }^{+}$, the amount of nickel(III) species varies. This can be explained by different traces of $\mathrm{HCl}$ within the $\mathrm{CH}_{2} \mathrm{Cl}_{2}$, due to slow autoxidation. ${ }^{[173,174]}$ When an EPR spectrum of a freshly prepared sample of $\mathbf{L N i}_{\mathbf{2}}{ }^{+}$ in freshly distilled $\mathrm{CH}_{2} \mathrm{Cl}_{2}$ was recorded, hardly any nickel(III) was found (Fig. 6.3), like observed by BLUSCH. ${ }^{[5]}$ With the addition of $\mathrm{Bu}_{4} \mathrm{NCl}$ the oxidation locus could be shifted nearly quantitatively towards a nickel(III) species (Fig. 6.3), due to axial binding of the chloride, stabilizing the nickel(III) species. This further reinforces the assumption on a ligand-centered oxidation locus in $\mathbf{L N i C o}^{+}$before addition of $\mathrm{Bu}_{4} \mathrm{NCl}$. 


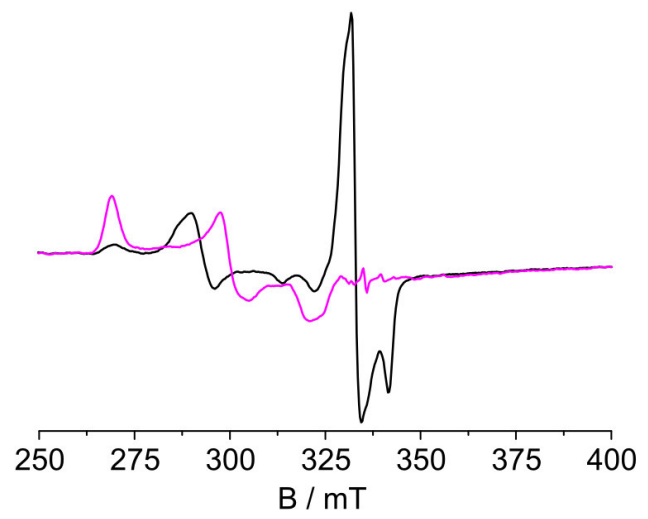

(a)

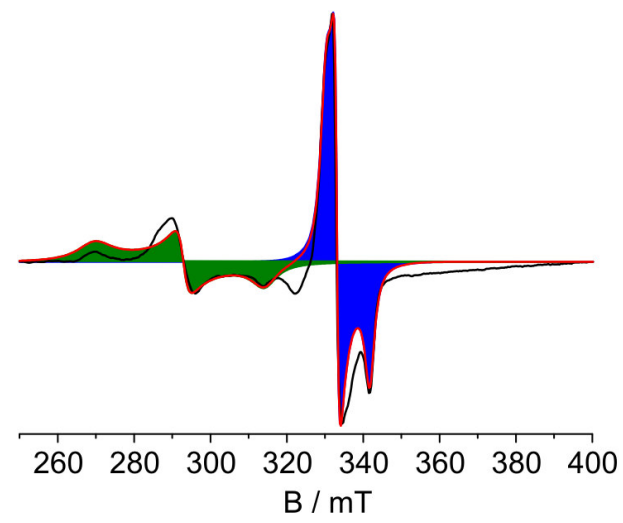

(b)

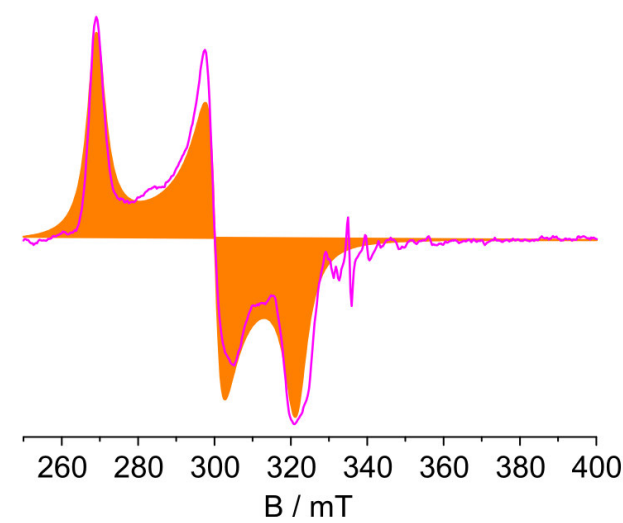

(c)

Figure 6.3: (a) EPR spectra of $\mathbf{L N i}_{2}{ }^{+}$in $\mathrm{CH}_{2} \mathrm{Cl}_{2}$ at $150 \mathrm{~K}$ before (black) and after (pink) addition of $\mathrm{Bu}_{4} \mathrm{NCl}$. (b) Simulated EPR spectrum (red) of $\mathbf{L N i}_{2}{ }^{+}$(black) with $\mathrm{g}_{x}=1.9655, \mathrm{~g}_{y}=2.0167, \mathrm{~g}_{z}=2.0344$ (Gaussian line shape, line width: $\mathrm{W}_{x}=35 \mathrm{G}$, $\mathrm{W}_{y}=19 \mathrm{G}, \mathrm{W}_{z}=43 \mathrm{G}$ ) for the organic radical (blue) and $\mathrm{g}_{x}=2.1382, \mathrm{~g}_{y}=2.2932$, $\mathrm{g}_{z}=2.4915$ (Gaussian line shape, line width: $\mathrm{W}_{x}=92 \mathrm{G}, \mathrm{W}_{y}=50 \mathrm{G}, \mathrm{W}_{z}=130 \mathrm{G}$ ) for the nickel(III) species (green) with an amplitude ratio of 1:2. (c) Simulated EPR spectrum (orange) of the nickel(III) species of $\mathbf{L N i}_{\mathbf{2}}{ }^{+}$after the addition of $\mathrm{Bu}_{4} \mathrm{NCl}$ with $\mathrm{g}_{x}=2.0900, \mathrm{~g}_{y}=2.2370, \mathrm{~g}_{z}=2.4958$ (Gaussian line shape, line width: $\mathrm{W}_{x}=80 \mathrm{G}, \mathrm{W}_{y}=60 \mathrm{G}, \mathrm{W}_{z}=65 \mathrm{G}$ ).

With the addition of $\mathrm{Bu}_{4} \mathrm{NPF}_{6}$ no change of the EPR spectra could be obeserved, indicating the effect only relies on the potential axial chloride ligand.

The oxidation of LNiCo is not unexpected even under inert conditions in an inert glove box. Cobalt(II) porphyrins are known to have rather low oxidation potentials in contrast to the corresponding complexes with different dicationic metal ions like nickel(II) and copper(II). The actual potential of cobalt(II)/cobalt(III) can vary in a wide range, depending on the electronic structure of the porphyrin ${ }^{[58,175]}$ but is always higher oxidation towards cobalt(III) is harder accessible - than for iron(II)/iron(III) within the same porphyrin (CoTPP: $0.32 \mathrm{~V}^{[176]}$; FeTPP: $-0.80 \mathrm{~V}^{[114]}$ vs. $\mathrm{Fc} / \mathrm{Fc}^{+}$(converted with $E\left(\mathrm{Fc} / \mathrm{Fc}^{+}\right)=0.46 \mathrm{~V}$ vs. $\left.\left.\mathrm{SCE}^{[141]}\right)\right)$. The rapid oxidation of $\mathbf{L N i C o}$ further explains 
why the corresponding Fe(II) complex of the Siamese-twin porphyrin could directly not be synthesized in a pure ferrous oxidation state (see. Chap. 5.1.5).

Interestingly the oxidized cobalt nickel complex could not be stabilized by addition of chloride, forming LNiCoCl, whereas the corresponding iron(III) complex LNiFeCl was stable under ambient conditions (Chap. 5.1). This is not a suprise, considering that decomposition under inert conditions was observed for a variety of cobalt(III) porphyrins. ${ }^{[8,32,146]}$

\subsection{Dicobalt Complex $\mathrm{LCo}_{2}$}

Following the procedure developed for $\mathbf{L N i C o}$ the corresponding dicobalt complex $\mathbf{L C \mathbf { C o } _ { 2 }}$ was synthesized with the free base Siamese-twin porphyrin $\mathbf{L H}_{\mathbf{4}}$ as starting point.

\subsubsection{Synthesis of $\mathrm{LCo}_{2}$}

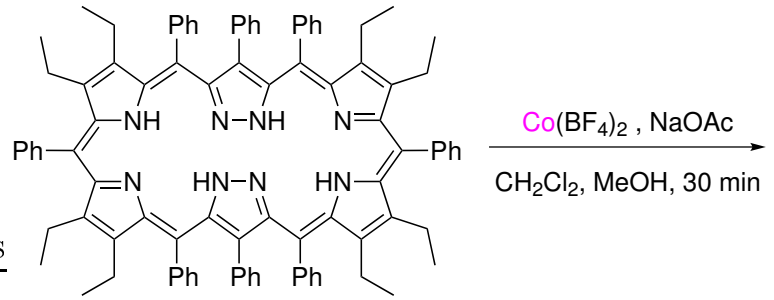

$\mathrm{LH}_{4}$

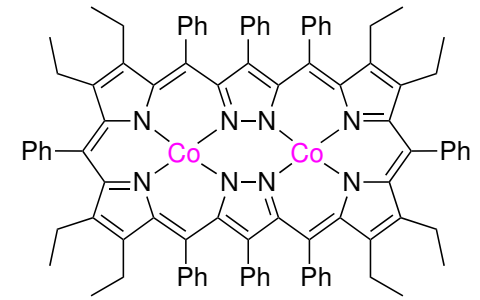

$\mathrm{LCo}_{2}$

Scheme 6.3: Synthesis of the homobimetallic complex $\mathbf{L C o}_{2}$.

$\mathrm{Co}\left(\mathrm{BF}_{4}\right)_{2}$ and $\mathrm{NaOAc}$ were added to a solution of the free base Siamese-twin porphyrin $\mathbf{L H}_{4}$ in polar solvent under inert conditions. The reaction mixture was stirred at ambient temperatures for 30 minutes. The solvent was removed under reduced pressure and the residue was filtered over a plug of Sephadex ${ }^{\mathrm{TM}}$, yielding $\mathbf{L} \mathbf{C o}_{2}$.

\subsubsection{Characterization of $\mathrm{LCo}_{2}$}

$\mathbf{L C o}_{2}$ could be characterized by HRMS (Fig. 6.1) but showed the expected decomposition even faster than LNiCo in solution as well as in solid state.

The higher instability of $\mathbf{L} \mathbf{C o}_{2}$ was expected and can be explained by once further oxidation in contrast to $\mathbf{L N i C o}{ }^{+}$, resulting in $\mathbf{L C o}_{2}{ }^{2+}$. It has been shown that even for the monocobalt complex LNiCo oxidation led to rapid decomposition. This decomposition is accelerated upon a second oxidation, like it has been observated for the chemically and 


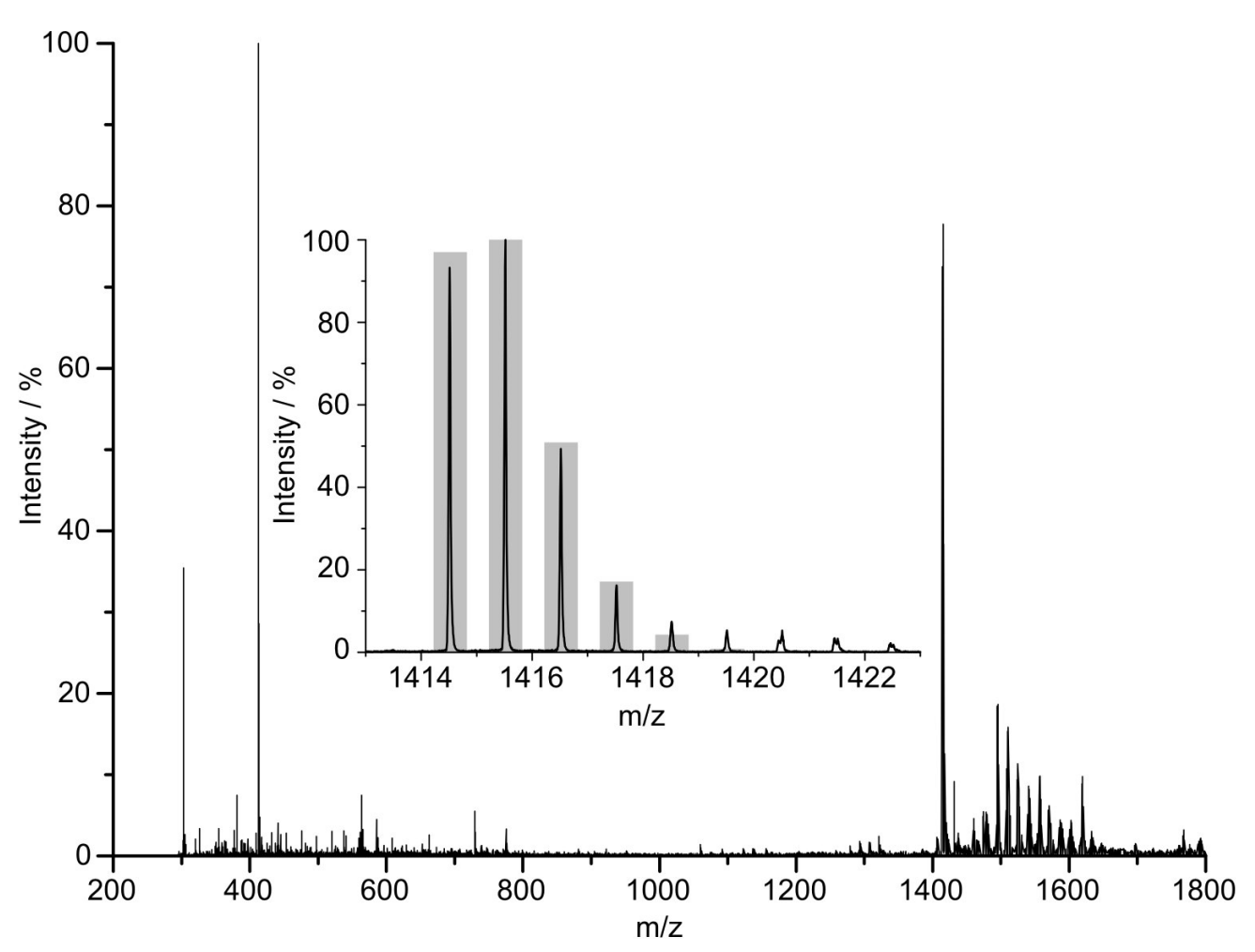

Figure 6.4: $\mathrm{HRMS} \mathrm{ESI}^{+}$spectrum of $\mathbf{L} \mathbf{C o}_{2}$ in $\mathrm{MeOH}$. Inset: Comparison of the measured (black) and calculated isotopic pattern (grey bars). $[\mathrm{M}]^{+} \mathrm{m} / \mathrm{z}=1414.5164$ (calcd.: 1414.5164).

electrochemically doubly oxidized metal complexes of $\mathbf{L} \mathbf{C u}_{2}, \mathbf{L N i C u}$ and $\mathbf{L N i}_{2}{ }^{[55]}$ and the monoxidized complex $\mathbf{L N i F e C l}{ }^{+}$(Chap. 5.1.3). 


\section{Preliminary Reactivity Studies of LNiFe and LNiFeCl}

First preliminary reactivity studies with the well characterized and stable complex LNiFeCl and its ferrous pendant LNiFe were performed to obtain first insights into their potential for substrate activation. Therefore, a selection of small molecules, or a corresponding group transfer agent, have been added to the ferrous and ferric iron nickel complexes in solution.

Iron complexes, not even necessarily of porphyrins, are known to react with different gases like CO, $\mathrm{NO}$ or $\mathrm{O}_{2} \cdot{ }^{[8,152,158,160,177]}$ Therefore, solutions of $\mathbf{L N i F e C l}$ and $\mathbf{L N i F e}$ were treated with these gases at different temperatures ranging from ambient temperature down to $-60{ }^{\circ} \mathrm{C}$. The complexes either decomposed while they were stirred with a gas for a longer time even at low temperature, or did not react at all. Possible intermediates of the decomposition reactions could not be isolated and characterized. Decomposition either means that the complex is successfully coordinating the small molecule but decomposes in a subsequent reaction, or the gas directly reacts with the porphyrin ring, leading to its breakdown. Neither of these two possibilities could be proven. The decomposition products likely did not retain a porphyrin like structure as deduced from UV-vis spectroscopy. NMR spectroscopic investigations always showed a variety of signals, mainly ethyl and phenyl groups, making NMR spectroscopy unsuitable to distinguish the breakdown product.

Similar behavior was observed when the complexes were treated with soluble iodosobenzene, an oxygen atom transfer agent, or $\mathrm{Ph}_{3} \mathrm{CSNO}$, a known NO transfer agent. ${ }^{[178,179]}$ Whereas $\mathbf{L N i F e C l}$ and $\mathbf{L N i F e}$ decomposed upon addition of the soluble iodosobenzene, they did not react with $\mathrm{Ph}_{3} \mathrm{CSNO}$ at all.

With the addition of $\mathrm{KO}_{2}$, a reagent that is regularly used to reduce ferric iron complexes and/or to form (su)peroxo complexes, ${ }^{[148,150]}$ to $\mathbf{L N i F e}$ a change of the UV-vis spectrum could be observed (Fig. 7.1).

After the addition of $\mathrm{KO}_{2}$ to a solution of $\mathbf{L N i F e C l}$ in THF, a reduction towards $\mathbf{L N i F e}$ (red trace) could be observed (cf. Fig. 5.11), followed by a slow reaction over the course of one hour to a second species (blue trace) with a new absorption band at $580 \mathrm{~nm}$. Similar observations have previously been made for different iron porphyrin complexes and have been explained with the coordination of an additional superoxide ion to the reduced iron(II) ion to yield an iron(III) peroxo adduct ${ }^{[17,148,149,180-184]}$ or an 


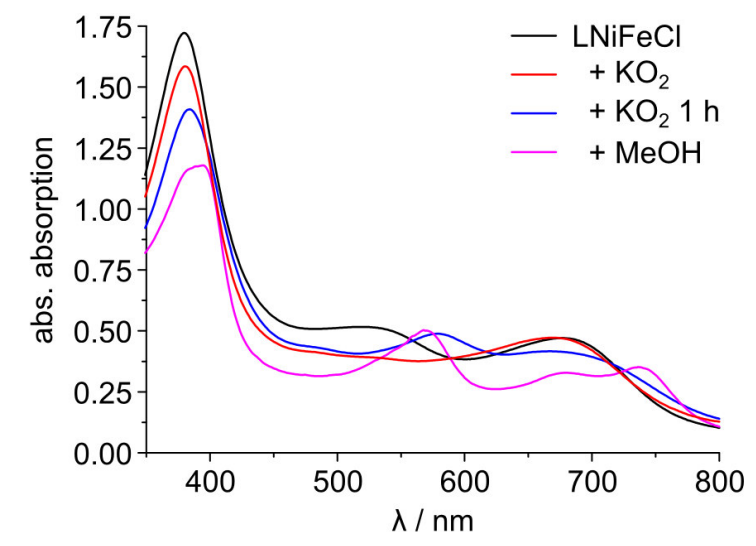

Figure 7.1: UV-vis spectra of $\mathbf{L N i F e C l}$ before (black) and after the addition of $\mathrm{KO}_{2}$ before (red and blue) and after addition of $\mathrm{MeOH}$ (pink) in THF at ambient temperature.

iron(II) superoxo adduct ${ }^{[149,183]}$ (Fig. 7.2). The observed absorption bands for these iron (su)peroxo adduct are within a region of 570-595 $\mathrm{nm}$ and are in good agreement with the observed absorption band of $580 \mathrm{~nm}$ for the blue trace in figure 7.1. In particular, for a substituted [meso-tetrakis(2,4,6-trimethylphenyl)-porphyrinato]iron(II) complex with an additional imidazole ( $\operatorname{Im})$ based ligand $([(\mathrm{tmp}) \operatorname{ImFe}])$ it recently has been shown that a transformation of the iron (su)peroxo adduct towards an Fe(III) hydroperoxo occurred, when $\mathrm{MeOH}$ was added as proton source (Fig. 7.2). ${ }^{[17]}$ Both, the (su)peroxo and hydroperoxo complex of [(tmp) ImFe] have been characterized spectroscopically using resonance raman, Mößbauer, UV-vis and EPR spectroscopy but no molecular structure could be obained so far. ${ }^{[17]}$

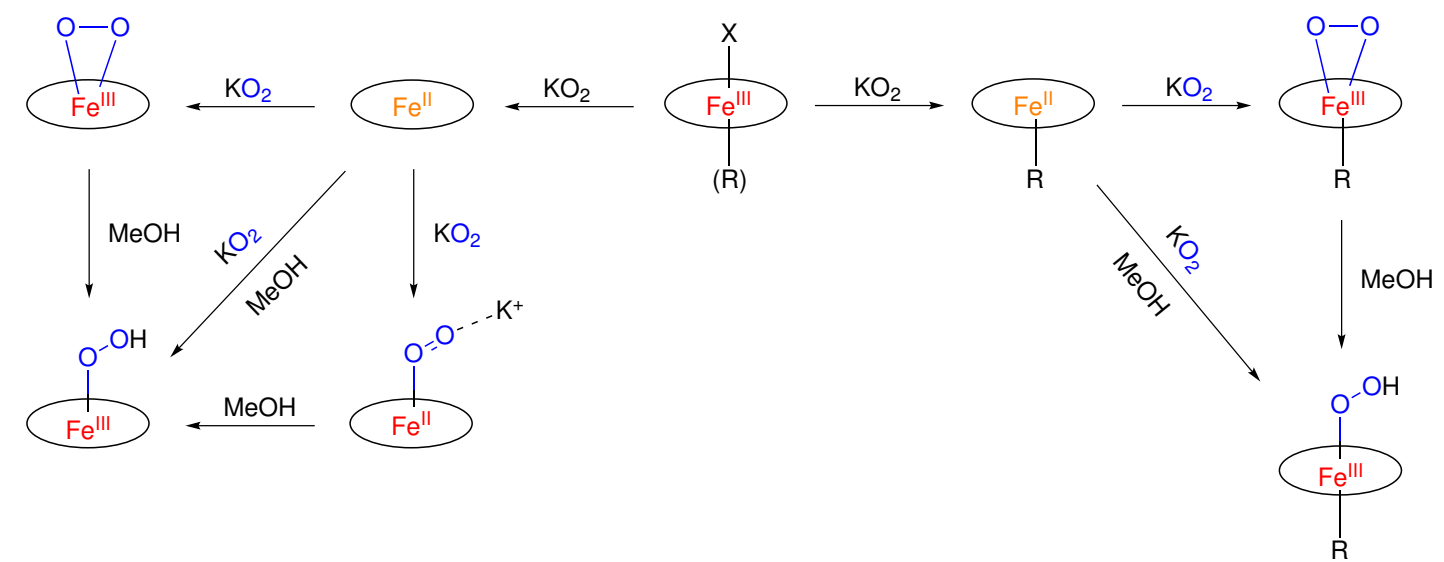

Figure 7.2: Proposed reaction of $\mathrm{KO}_{2}$ with a five coordinated iron(III) porphyrin (left) and a six coordinated iron(III) porphyrin with a covalently linked ligand $\mathbf{R}$ (right). ${ }^{[17,148,149,180-184]}$

Upon the addition of $\mathrm{MeOH}$ to the solution of $\mathbf{L N i F e}$ and $\mathrm{KO}_{2}$, a further change in the UV-vis spectrum could be observed (Fig. 7.1 pink trace): The new absorption band at around $570 \mathrm{~nm}$ is shifted to lower wavelength and is within the same region as the assumed hydroperoxo absorption band for [(tmp)ImFe]. ${ }^{[17]}$ To investigate if the pink trace in Fig 7.1 could correspond to a hydroperoxo adduct, ferrous $\mathbf{L N i F e}$ was synthesized with the 
use of $\mathrm{KO}_{2}$, whose excess was filtered off, and the solution was split into two portions. The first one was treated with $\mathrm{KO}_{2}$ and $\mathrm{MeOH}$, whereas the second one was treated with tert-butyl-hydroperoxide ( $t$-BuOOH), a known hydroperoxide transfer reagent.

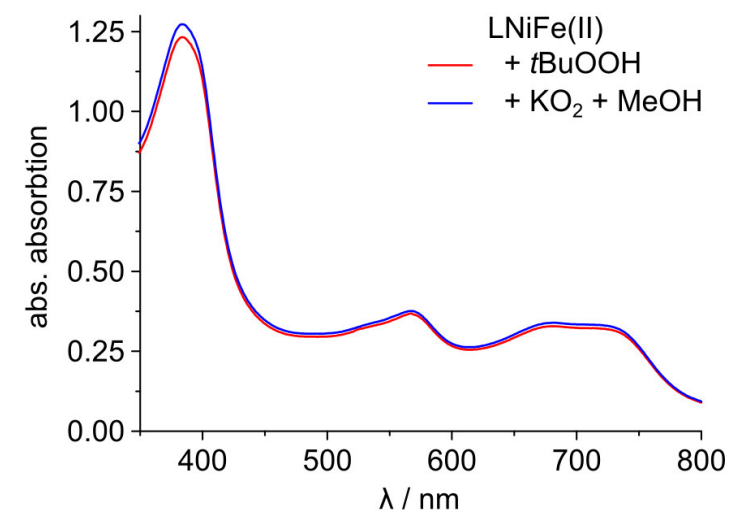

Figure 7.3: UV-vis spectra of $\mathbf{L N i F e}$ after the addition of $\mathrm{KO}_{2}$ and $\mathrm{MeOH}$ (blue) and after the addition of $t$ - $\mathrm{BuOOH}$ (red) in THF at ambient temperature.

The addition of $\mathrm{KO}_{2}$ and methanol as well as the addition of $t$-BuOOH led to the formation of exactly the same UV-vis spectrum (Fig. 7.3), suggesting formation of the same species (eventually a hydroperoxo complex LNiFeOOR, $\mathrm{R}=\mathrm{H}, t$-Bu). The reaction with $\mathrm{KO}_{2}$ and $t$-BuOOH was repeated with $\mathbf{L} \mathbf{N i}_{\mathbf{2}}$ and $\mathbf{L} \mathbf{C u}_{\mathbf{2}}$ to inverstigate if the change in the UV-vis spectrum primary resulted from a decomposition of the STP framework. Whereas the dinickel complex showed no reaction with $\mathrm{KO}_{2}$ and $t$ - $\mathrm{BuOOH}$, the corresponding dicopper complex did (Fig. 7.4).

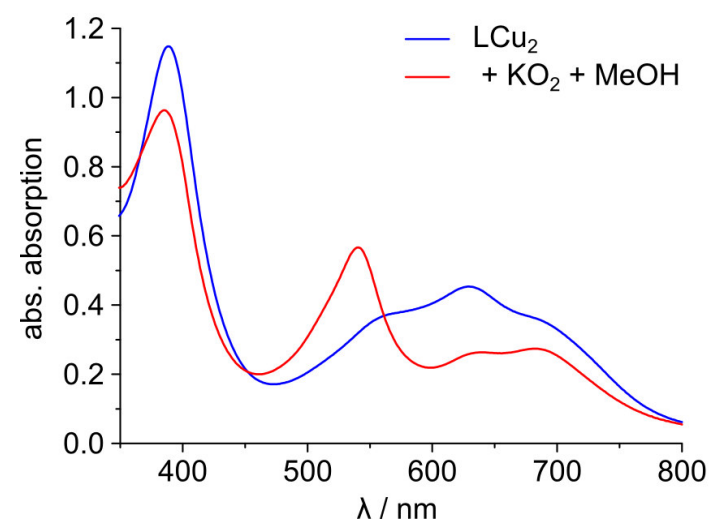

(a)

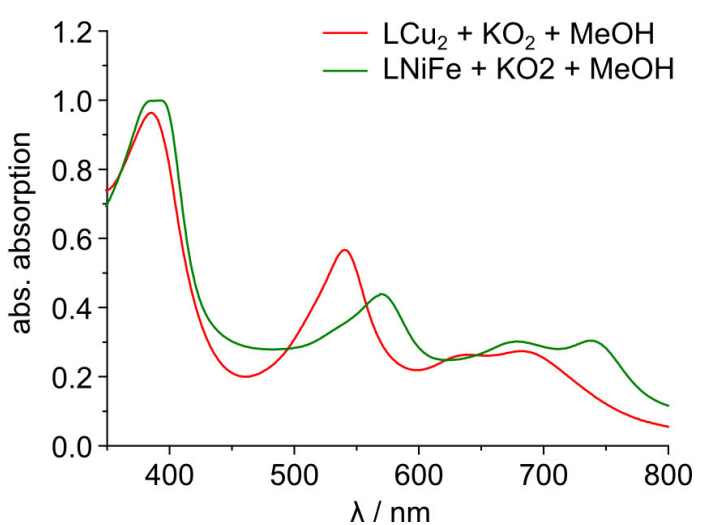

(b)

Figure 7.4: (a) UV-vis spectra of $\mathbf{L C} \mathbf{C u}_{2}$ before (blue) and after (red) the addition of $\mathrm{KO}_{2}$ and

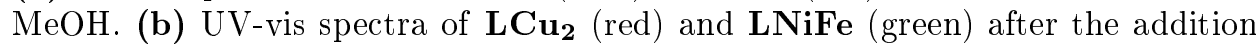
of $\mathrm{KO}_{2}$ and $\mathrm{MeOH}$. All spectra were measured in THF at ambient temperature.

$\mathbf{L C u}_{2}$ and $\mathbf{L N i F e}$ showed comparable spectra after the addition of $\mathrm{KO}_{2}$ and $\mathrm{MeOH}$. The slight shift of the absorption bands for presumed ( $\mathbf{L N i F e O O R}$ ) and $\left(\mathbf{L C u}_{2} \mathbf{O O R}\right)$ species can be explained with the different electronic structure of the copper and iron ions within the complexes. 
However, further studies have to be performed to investigate the outcome of the reaction with $\mathrm{KO}_{2}$ and $(t-\mathrm{BuOOH})$ and to probe whether any adducts are formed or not.

Especially the ferric complex LNiFeOOH would be interesting, because ferric hydroperoxo species of an iron porphyrin have been supposed to be the intermediate in the formation of Cpd I from the peroxo species in heme enzymes like Cyt P450 (cf. Fig. 1.3). Furthermore, the hydroperoxo species of Cyt P450, also called Cpd 0, was discussed to be the active species in Cyt P450. ${ }^{[185,186]}$ Even though Cpd 0 has been characterized spectroscopically, no suitable model complex could be synthesized so far. ${ }^{[184,185]}$ 


\section{Summary and Outlook}

In conclusion, a variety of new Siamese-twin porphyrins, their precursors and their dicopper complexes could be synthesized and the effet of different STP substituents on redox potentials and spectroscopic properties of the complexes was investigated. Furthermore, different homo- and heterobimetallic iron complexes of the parent STP were synthesized, and their coordination chemistry and electronic structure was investigated in comprehensive studies.

In a first step, the substituents at the pyrazole's meso-positions of the STP were varied and the pyrazole building blocks 18, 20, 21 and 22, comprising a pyrazole and two phenylacetylene units, were developed and synthesized. A collection of different coupling reactions has been performed to attach $\mathbf{1 8}, \mathbf{2 0}, \mathbf{2 1}$ and $\mathbf{2 2}$ to 3,5-diethyl-pyrrole and various other heterocycles. None ot these reactions led to any coupling product.

In a second approach, the meso-phenyl group located between two pyrrolic moieties was varied. The resulting expanded porphyrins $\mathbf{x}_{\mathbf{L H}}$, with electron donating and withdrawing substituents, were characterized and showed no conformational difference compared to the parent STP. The helical conformation was retained, as could be shown by single crystal X-ray diffraction for ${ }^{p \mathbf{M e}} \mathbf{L H}_{\mathbf{4}}$ and ${ }^{p \mathbf{F}} \mathbf{L H}_{\mathbf{4}}$, even though they crystallized in a different space group, and by DFT calculations for ${ }^{\mathrm{MeO}} \mathbf{L H}_{\mathbf{4}}$ and $p \mathrm{MeO}_{\mathrm{LH}_{4}}$.

Likewise, the influence on the electronic structure of the free base STPs, as assessed by their near-identical optical spectra, is minute. However, the influence of the substituents on the yield of the STP synthesis, their solubility, and degree of crystallinity is remarkably large. Particularly the meso-bistolyl-tetraphenyl-derivative ${ }^{p \mathbf{M e}} \mathbf{L H}_{\mathbf{4}}$ recommends itself for its ease of synthesis in relatively large scales, solubility, relative chemical stability, and crystallinity for further investigation of the chemistry of the STPs.

The influence of the different electron donating or withdrawing aryl groups was further investigated for the copper complexes ${ }^{\mathbf{X}} \mathbf{L} \mathbf{C u}_{\mathbf{2}}$ of the new STPs. The difference in the electronic structure of the copper complexes, as could be observed by UV-vis spectroscopy, was minute. The oxidation potentials of the copper complexes were somewhat more affected by the derivatizations, reflecting their influence on the oxidation site, namely the dipyrromethene subunits to which the substituents are attached. With the electron withdrawing fluorine substituent the potentials were shifted to higher values, whereas they were shifted to lower potentials with the electron donating substituents, 
with the shifts conforming to linear Hammett plots. Interestingly, the exchange of one or both copper ions with one or two nickel ions has a much bigger influence on the corresponding redox potentials than the substitution patterns of the two meso-aryl groups. Even though the electronic influence by changing two of eight substituents is minor, it is not negligible and can help to finetune the redox properties of metal complexes of the STPs.
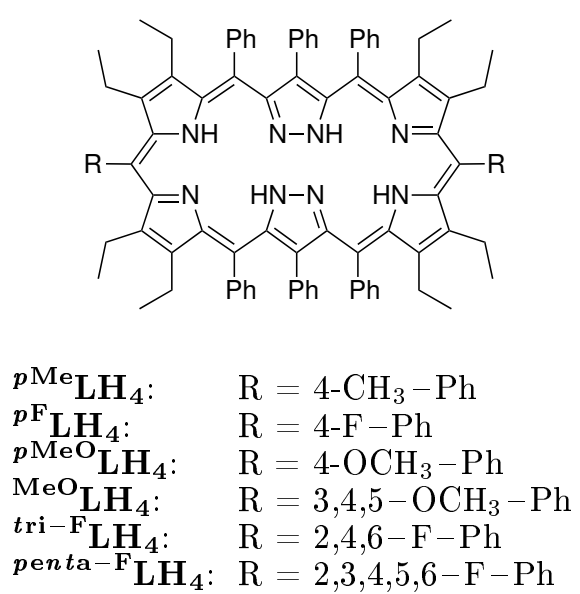

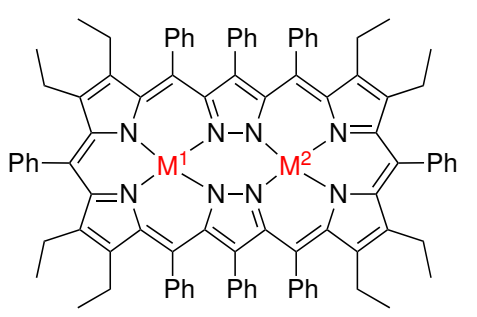

$\mathbf{L H}_{2}$ FeCl: $\quad \mathrm{M}^{1}=2 \mathrm{H}, \mathrm{M}^{2}=\mathrm{FeCl}$

LCuFeCl: $\quad \mathrm{M}^{1}=\mathrm{Cu}, \mathrm{M}^{2}=\mathrm{FeCl}$

LNiFeCl: $\quad \mathrm{M}^{1}=\mathrm{Ni}, \mathrm{M}^{2}=\mathrm{FeCl}$

LNiFe: $\quad \mathrm{M}^{1}=\mathrm{Ni}, \mathrm{M}^{2}=\mathrm{Fe}$

$\mathrm{L}\{\mathbf{F e C l}\}_{2}: \mathrm{M}^{1}=\mathrm{M}^{2}=\mathrm{FeCl}$

LNiCo: $\quad \mathrm{M}^{1}=\mathrm{Ni}, \mathrm{M}^{2}=\mathrm{Co}$

LCo $\mathbf{C o}_{2}: \quad \mathrm{M}^{1}=\mathrm{M}^{2}=\mathrm{Co}$

Furthermore, the fundamental iron coordination chemistry of the parent Siamese-twin porphyrin was investigated with different homo- and heterobimetallic iron complexes. The direct synthesis of ferrous complexes proved to be unachievable, due to partial oxidation to the ferric complexes. The synthesis of ferric complexes $\mathbf{L H}_{\mathbf{2}} \mathbf{F e C l}, \mathbf{L C u F e C l}$, $\mathbf{L N i F e C l}$ and $\mathbf{L}\{\mathbf{F e C l}\}_{2}$ was notably easier.

The unique conformation of the STP scaffold is retained upon exchange of a square planar coordinated metal ion (nickel(II), copper(II)) with a square pyramidal coordinated metal ion (iron(III)), as could be shown by single crystal X-ray diffraction of $\mathbf{L N i F e C l}$ and $\mathbf{L}\{\mathbf{F e C l}\}_{2}$. With this unique conformation and coordination motif of the STP in combination with the weakly binding chloride in the iron ion's axial position, splitting of the molecular orbitals was within the perfect range to favor a pure $\mathrm{S}=3 / 2$ intermediate spin state for the iron(III) ion in $\mathbf{L C u F e C l}, \mathbf{L N i F e C l}$ and $\mathbf{L}\{\mathbf{F e C l}\}_{2}$. This intermediate spin state was confirmed by EPR and Mößbauer spectroscopy and magnetic susceptibility measurements. Mößbauer spectra of all bimetallic iron complexes always showed two doublets. Whereas the first one, with a large quadrupole splitting of around 2.80 and a quantity of $80 \%$ was in agreement with an is-iron(III) ion, the other one had an untypically small quadrupole splitting for an is-iron(III) signal, in a quantity of $20 \%$ for $\mathbf{L N i F e C l}$ and $\mathbf{L C u F e C l}$, and a $50 \%$ quantity for $\mathbf{L}\{\mathbf{F e C l}\}_{\mathbf{2}}$. The ratio of both Mößbauer doublets was retained at any temperature measured and was further investigated for $\mathbf{L N i F e C l , ~ t h e ~ m o s t ~ s t a b l e ~ i r o n ~ c o m p l e x ~ o f ~ t h e ~ S T P . ~ I n ~ c o o r d i n a t i n g ~}$ (THF) and non-coordinating (toluene) solvents the ratio of the two Mößbauer doublets of 1:4 was retained and furthermore reappears after reoxidation of the ferrous complex 
LNiFe. Thus, the second Mößbauer doublet is definitely part of the interesting spin system of ferric iron complexes of the STP, but could not be explained so far.

The redox chemistry of $\mathbf{L C u F e C l}, \mathbf{L N i F e C l}$ and $\mathbf{L}\{\mathbf{F e C l}\}_{2}$ was studied by cyclic and square wave voltammetry. Besides the previously observed ligand-based oxidation and reduction events, cyclic and square wave voltammetry of $\mathbf{L C u F e C l}, \mathbf{L N i F e C l}$ and $\mathbf{L}\{\mathbf{F e C l}\}_{2}$ showed an additional accessible reduction wave for the iron(III) ion at around $-1.10 \mathrm{~V}$ vs. $\mathrm{Fc} / \mathrm{Fc}^{+}$. All iron complexes were more prone to decompose upon oxidation, but were found to be stable under aerobic conditions. However, LNiFeCl could be oxidizied with $\mathrm{AgBF}_{4}$ under anaerobic conditions and showed the expected STP scaffold based oxidations, as could be observed by instantaneous EPR spectroscopy. Furthermore, the first ferrous iron complex $\mathbf{L N i F e}$ was synthesized using $\mathrm{CoCp}_{2}$ as a reductant, which resulted in a clean conversion to a hs-iron(II) complex, with only one Mößbauer doublet. LNiFe was reoxidized with the addition of an oxidizing reagent ( $m$-CPBA) and the ratio of 4:1 for the two Mößbauer doublets for the ferric complex was recovered.

Finally, first preliminary reactivity studies with LNiFe were performed, clearly showing that the iron complexes of the STP are much distinct from their porphyrin congeners and do not react in a straightforward way with typical substrates such as $\mathrm{CO}, \mathrm{NO}$ or $\mathrm{O}_{2}$.

Now, that the fundamental coordination chemistry of iron complexes of the STP is investigated, the iron complexes of the $\operatorname{STP}(\mathrm{s})$ can be studied in a more detailed fashion, concerning for example reactivity studies. Furthermore, with the use of the synthesized monoiron complex $\mathbf{L H}_{2} \mathbf{F e}$ a variety of different metal complexes incorporating different redox active metal ions can be synthesized, to maybe find suitable complexes for substrate activation. 



\section{Experimental Section}

\subsection{Instruments and Materials}

\section{NMR Spectroscopy}

NMR spectra were recorded on Bruker Avance $400 \mathrm{MHz}$ or $500 \mathrm{MHz}$ spectrometers at room temperature if not mentioned otherwise. ${ }^{13} \mathrm{C}$ NMR spectra were generally recorded in proton decoupled mode. Chemical shifts are reported in ppm relative to residual proton signals of the solvent. The multiplicity of the signals is abbreviated as follows: singlet (s), doublet (d), triplet (t), quartet (q), quintet (qu), multiplet (m), broad signal (br). The coupling constant is abbreviated as follows, depending on the range of the coupling: ${ }^{1} J,{ }^{2} J,{ }^{3} J,{ }^{4} J$.

\section{Mass Spectrometry}

Mass spectrometry measurements were recorded on a Bruker APEX IV (FTICR-MS), a Bruker MicroTOF (ESI-TOF-MS) or a Bruker maXis (ESI-QTOF-MS) spectrometer. Methanol was used as solvent if not mentioned otherwise.

$[\mathrm{M}]$ indicates the corresponding organic molecule or metal complex without any axial ligand.

\section{Infrared Spectroscopy}

IR spectra were recorded on a Brucker VERTEX 70 spectrometer using $\mathrm{KBr}$ pellets.

The position of the reported bands is given in wavenumbers $\left(\mathrm{cm}^{-1}\right)$ and the reported intensity is abbreviated as follows: very strong (vs), strong (s), medium (m), weak (w).

\section{UV-vis Spectroscopy}

UV-vis spectra were recorded at room temperature on a Varian Cary 5000 spectrophotometer using quartz cuvettes $(d=1 \mathrm{~cm})$. Dichloromethane was used as solvent if not mentioned otherwise.

The position of the reported bands is given in $\mathrm{nm}$ and the extinction coefficient is given in $\mathrm{L} \cdot \mathrm{mol}^{-1} \cdot \mathrm{cm}^{-1}$.

\section{Mößbauer Spectroscopy}

Mößbauer spectra were recorded on a Mößbauer spectrometer with a helium closed cycle cryostate and a ${ }^{57} \mathrm{Co}$ source. The isomere shift $(\delta)$ is given relative to $\alpha$-iron foil. The 
program Mfit (E. Bill, Max-Planck Institute for Chemical Energy Conversion, Mülheim an der Ruhr, Germany, 2008) was used for simulations.

\section{Susceptibility Measurements}

Susceptibility measurements were performed on a Quantum-Design MPMS-5S SQUID magnetometer equipped with a 5 Tesla magnet. The powdered sample was contained in a teflon bucket and fixed in a non-magnetic sample holder. The raw data file of the measured magnetic moment was corrected for the diamagnetic contribution of the sample holder and the teflon bucket. Simulation of the experimental data with a fullmatix diagonalization of exchange coupling and Zeeman splitting was perfomed with the julX program (E. Bill, Max-Planck Institute for Chemical Energy Conversion, Mülheim an der Ruhr, Germany, 2008).

\section{Elemental Analysis}

Standard CHN elemental analyses were measured on an Elementar 4.1 vario EL 3 by the "Analytic Laboratory" of the Institute of Inorganic Chemistry at the University of Göttingen.

\section{X-Ray Crystallography}

X-ray data were collected on a STOE IPDS II diffractometer (graphite monochromated Mo- $\mathrm{K}_{\alpha}$ radiation, $\lambda=0.71073 \AA$ ) by use of $\omega$ scans. The structures were solved with SHELXT ${ }^{[187]}$ and refined on $F^{2}$ using all reflections with SHELXL-2014. ${ }^{[188]}$ All nonhydrogen atoms were refined anisotropically. Most hydrogen atoms were placed in calculated positions and assigned to an isotropic displacement parameter of $1.2 / 1.5 \mathrm{U}_{\text {eq }}(\mathrm{C})$. Nitrogen-bound hydrogen atoms were refined freely.

\section{Electron Paramagnetic Resonance Measurements}

X-band EPR measurements in the temperature range of 120-198 K were performed on a Bruker ELEXSYS E500 spectrometer equipped with a ER-049X microwave bridge, an ER083CS magnet, a digital temperature control system ER 4131 VT and liquid nitrogen as coolant. Measurements at lower temperatures were performed on a Bruker ELEXSYS E500 spectrometer equipped with a ER-049X microwave bridge, an ER032T magnetic field controller calibrated with a Bruker ER035M NMR field probe, a Bruker ER4116DM dual-mode or a ER4102ST standard cavity and an Oxford Instruments ESR 900 helium flow cryostat at the Max-Planck Institute for Chemical Energy Conversion in Mülheim an der Ruhr. The microwave frequency of $9.4 \mathrm{GHz}$ was modulated with 5-9 G field modulation amplitude, $100 \mathrm{kHz}$ field modulation frequency and a microwave power of around $10 \mathrm{~mW}$. EPR simulations have been done with the programs esim_gfit and esim_sx (E. Bill, Max-Planck Institute for Chemical Energy Conversion, Mülheim an der Ruhr, Germany, 2008).

\section{Electrochemical Measurements}

Electrochemical measurements were performed in dichloromethane with $\left[\mathrm{NBu}_{4}\right] \mathrm{PF}_{6}$ 
(0.1 M) as electrolyte using a Perkin Elmer 263A potentiostat controlled by Electrochemistry Powersuite software (Princeton Applied Research). A glassy carbon electrode was used as working electrode, together with a platinum wire counter and a silver wire reference electrode. Decamethylferrocene was added as internal standard $\left(\mathrm{E}_{1 / 2}=-0.59 \mathrm{~V}\right.$ vs $\left.\mathrm{Fc} / \mathrm{Fc}^{+}\right)^{[141]}$ after the measurement. Bulk electrolysis were performed in dichloromethane with $\left[\mathrm{NBu}_{4}\right] \mathrm{PF}_{6}(0.1 \mathrm{M})$ as electrolyte and a Metrohm Autolab PGSTAT101 controlled by Novasoft Software using a platinum net as working electrode, together with a platinum wire counter and a silver wire reference electrode.

\section{Thin Layer Chromatography}

Thin Layer Chromatography was carried out on fluorescence active polyester sheets coated with silica gel Machery-Nagel MN60 (Polygram ${ }^{\circledR}$ SIL G/UV $254,0.2 \mathrm{~mm}$ silica).

\section{Column Chromatrography}

Column chromatography was performed on Machery-Nagel MN60 (0.063-0.2 mm/70230 mesh ASTM) silica gel or Machery-Nagel MN90 (0.05-0.200 mm, BET $\sim 130 \mathrm{~m}^{2} / \mathrm{g}$, Brockmann activity 1) basic aluminum oxide.

Size exclusion chromatography was perfomed on GE Healthcare Sephadex ${ }^{\text {TM }}$ LH-20.

\section{DFT Calculations}

DFT calculations were carried out using the ORCA program (version 3.0.3) (F. Neese, ORCA, Max-Planck Institute for Chemical Energy Conversion, Mühlheim/Ruhr, Germany, 2012.). Atom coordinates were obtained from the crystal structure of the parent Siamese-twin porphyrin ${ }^{[52]}$ and refined using the Becke-Perdew-1986 functional (BP86) and the def2-tzvp basis set for the copper atoms and the def2-svp basis set for all other atoms. ${ }^{[53]}$ Structure optimizations of all compounds readily converged.

\section{Materials and Equipment}

Reactions were carried out under ambient conditions if not mentioned otherwise. Reactions under inert conditions were carried out under nitrogen or argon atmosphere using Schlenk techniques with appropriate glassware or in a glovebox.

Materials obtained from commercial suppliers (Sigmaaldrich, Fluka, VWR, Deutero, ABCR, TCI, Fisher, ACROS, Roth, Merck) were used without further purification, except benzaldehyde, which was freshyl distilled before usage.

Anhydrous solvents were dried and destillied according to literature procedures. ${ }^{[189]}$ Compounds $1 H$-pyrazole-3,5-dicarbaldehyde (16), 3,4-diethyl-pyrrole and the regular Siamese-twin porphyrin $\mathbf{9}$ were synthesized according to literature. ${ }^{[10,51,52,190]}$ 


\subsection{Synthesis of the Pyrazole Building Blocks 18, 19, 20, 21 and 22}

\subsubsection{1-(Methoxymethyl)-1 $H$-pyrazole-3,5-dicarbaldehyde (19)}

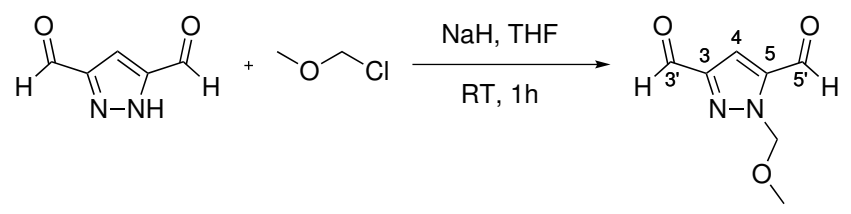

$1 H$-pyrazole-3,5-dicarbaldehyde $(2.00 \mathrm{~g}, 16.1 \mathrm{mmol}, 1.0 \mathrm{eq}$.) was dissolved in THF $(800 \mathrm{~mL})$ over night. A $60 \%$ dispersion of sodium hydride in mineral oil (1.93 g, $48.3 \mathrm{mmol}, 3.0$ eq.) was added and the mixture was stirred for $50 \mathrm{~min}$ at ambient temperature. Subsequently (Chloromethyl)methylether $(1.84 \mathrm{~mL}, 1.93 \mathrm{~g}, 24.2 \mathrm{mmol}$, 1.5 eq.) was added dropwise and the reaction mixture was stirred for another $50 \mathrm{~min}$ at ambient temperature. The reaction was stopped by addition of $500 \mathrm{~mL}$ of demineralized water. The mixture was extracted with dichloromethane $(3 \times 300 \mathrm{~mL})$, dried over sodium sulfate and the solvent was removed under reduced pressure. The raw product was purified by colum chromatography (silica, $n$-hexane/EtOAc $2: 1, \mathrm{R}_{f}=0.50$ ). The product was obtained as a yellow solid (1.61 g, $60 \%)$.

Empirical Formula:

Molecular Weight $(\mathrm{g} / \mathrm{mol})$ :

${ }^{\mathbf{1}} \mathbf{H}$ NMR (500 MHz, $\left.\mathrm{CDCl}_{3}\right)$ :

${ }^{13}$ C NMR (125 MHz, $\left.\mathrm{CDCl}_{3}\right)$ :

$\operatorname{MS}(\mathrm{EI}): m / z=$

IR $(\mathrm{KBr})$ :
$\mathrm{C}_{7} \mathrm{H}_{8} \mathrm{~N}_{2} \mathrm{O}_{3}$

168.15

$\delta / \mathrm{ppm}=3.38\left(\mathrm{~s}, 3 \mathrm{H}, \mathrm{CH}_{3}\right), 5.84\left(\mathrm{~s}, 2 \mathrm{H}, \mathrm{CH}_{2}\right)$, $7.45\left(\mathrm{~s}, 1 \mathrm{H}, \mathrm{H}^{\mathrm{pz}}\right), 9.95$ (s, 1H, C5'HO), 10.02 (s, $1 \mathrm{H}, \mathrm{C} 3$ 'HO).

$\delta / \mathrm{ppm}=57.5\left(\mathrm{CH}_{3}\right), 82.3\left(\mathrm{CH}_{2}\right), 114.5(\mathrm{C} 4)$, 140.9 (C5), 151.0 (C3), 179.6 (C5'), 185.7 (C3'). $168(12)[\mathrm{M}]^{+}, 153(26)\left[\mathrm{M}-C H_{3}\right]^{+}, 139$ (30) $[\mathrm{M}-\mathrm{CHO}]^{+}, 137(20)\left[\mathrm{M}-\mathrm{OCH}_{3}\right]^{+}, 125$ (9) [M$\mathrm{MOM}+2 \mathrm{H}]^{+}, 123(12)[\mathrm{M}-\mathrm{MOM}]^{+}, 81$ (7), 79 (9), $52(8), 45(100)[\mathrm{MOM}]^{+}$.

$\left.\widetilde{v}\left(\mathrm{~cm}^{-1}\right)=2950(\mathrm{~s}), 2589(\mathrm{~m}), 2360 \mathrm{w}\right)$, $2209(\mathrm{w}), 2013(\mathrm{w}), 1703$ (vs), 1535 (s), 1461 (s), 1385 (m), 1324 (s), 1246 (s), 1208 (s), 1105 (s), $1208(\mathrm{~s}), 1040(\mathrm{~m}), 999(\mathrm{~m}), 916(\mathrm{~s}), 846(\mathrm{~m})$, $792(\mathrm{~s}), 785(\mathrm{~s}), 758(\mathrm{~s}), 743(\mathrm{~s}), 589(\mathrm{~m})$, , $549(\mathrm{~m}), 534(\mathrm{~m})$. 


\subsubsection{1-(Methoxymethyl)-3,5-bis(1-hydroxy-3-phenylprop-2-yn-2-yl)-1H- pyrazole \\ (20)}

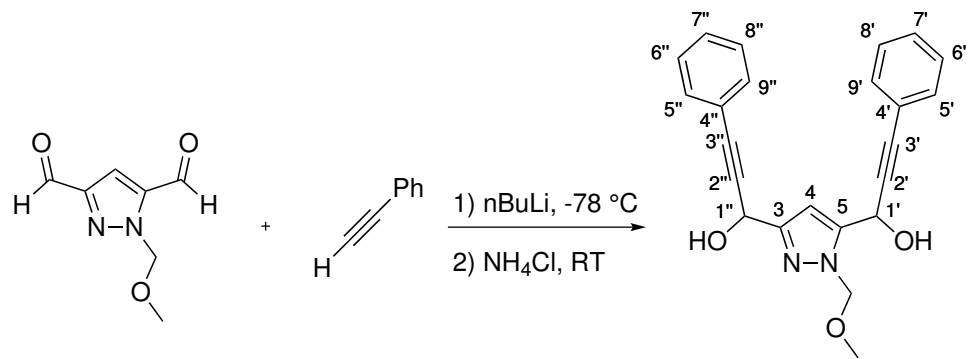

$n \operatorname{BuLi}(2.5 \mathrm{M}, 8.44 \mathrm{~mL}, 21.1 \mathrm{mmol}, 2.2$ eq.) was added to a solution of phenylacetylene (2.32 mL, $2.16 \mathrm{~g}, 21.1 \mathrm{mmol}, 2.2$ eq.) in dry THF $(100 \mathrm{~mL})$ at $-78{ }^{\circ} \mathrm{C}$ under inert conditions and the mixture was stirred for $1 \mathrm{~h}$. Subsequently 1-(methoxymethyl)- $1 \mathrm{H}$ pyrazole-3,5-dicarbaldehyde (19) $(1.61 \mathrm{~g}, 9.95 \mathrm{mmol}, 1.0$ eq.) in THF (100 mL) was added and the reactions mixture was allowed to warm up to ambient temperature under stirring for $1 \mathrm{~h}$. After addition of $200 \mathrm{~mL}$ of a saturaed aqueous solution of $\mathrm{NH}_{4} \mathrm{Cl}$ the mixture was extracted with THF $(3 \times 50 \mathrm{~mL})$, washed with brine $(100 \mathrm{~mL})$ and dried over sodium sulfate. The solvent was removed under reduced pressure and the raw product was purified by column chromatography (silica, $n$-hexane/EtOAc $1: 1, \mathrm{R}_{f}=0.29$ ). The product was obtained as a yellow solid (2.62 g, $73 \%)$.

Empirical Formula:

Molecular Weight $(\mathrm{g} / \mathrm{mol})$ :

${ }^{\mathbf{1}} \mathbf{H}$ NMR (500 MHz, acetone- $\left.\mathrm{d}_{6}\right)$ :

${ }^{13}$ C NMR (125 MHz, acetone- $\left.\mathrm{d}_{6}\right)$ :
$\mathrm{C}_{23} \mathrm{H}_{20} \mathrm{~N}_{2} \mathrm{O}_{3}$

372.42

$\delta / \mathrm{ppm}=3.33 \quad\left(\mathrm{~s}, \quad 3 \mathrm{H}, \quad \mathrm{CH}_{3}\right), \quad 5.46 \quad(\mathrm{~d}$, $\left.{ }^{3} J(\mathrm{H}, \mathrm{H})=10 \mathrm{~Hz}, 1 \mathrm{H}, \mathrm{CH}_{2}\right), 5.65(\mathrm{~s}, 1 \mathrm{H}$, 1 '- $\mathrm{H}), 5.67\left(\mathrm{~d}, 1 \mathrm{H}, \mathrm{CH}_{2}\right), 5.91\left(\mathrm{~s}, 1 \mathrm{H}, 1^{\prime}-\mathrm{H}\right)$, $6.72\left(\mathrm{~s}, 1 \mathrm{H}, \mathrm{H}^{\mathrm{pz}}\right), 7.35-7.37$ (m, 3H, 6"-H, 7"-H, 8'-H), 7.38-7.40 (m, 3H, 6'-H, 7'-H, 8'-H), 7.43-7.45 (m, 2H, 5"-H, 9"-H), 7.49-7.51 (m, 2H, 5'-H, 9'-H). (All signals are doubled due to the presence of diastereomeric pairs of enantiomers.) $\delta / \mathrm{ppm}=56.7\left(\mathrm{CH}_{3}\right), 56.8\left(\mathrm{C}^{\prime}\right), 59.9(\mathrm{C} 1 ”), 80.8$ $\left(\mathrm{CH}_{2}\right), 84.9$ (C3”), 85.5 (C3'), 88.7 (C2'), 90.8 (C2”), 105.3 (C4), 123.3 (C4'), 123.8 (C4"), 129.3 (C6", C8"), 129.4 (C7"), 129.4 (C7'), 129.7 (C6', C8'), 132.3 (C5”, C9”), 132.4 (C5', C9'), 144.7 (C5), 153.1 (C3). 
$\operatorname{MS}(\mathrm{EI}): m / z=$

$372(20)[\mathrm{M}]^{+}, 343(15)[\mathrm{M}-\mathrm{CHO}]^{+}, 341(15)$
$\left[\mathrm{M}-\mathrm{OCH}_{3}\right]^{+}, 340(15), 339(15), 327(36)[\mathrm{M}-$
$\mathrm{MOM}^{+}, 310(100)[\mathrm{M}-\mathrm{MOM}-\mathrm{OH}]^{+}, 281(66)$,
$265(7), 252(12), 241(9), 237(13), 225(16)$,
$209(24), 197(37), 195(14), 181(18), 169(10)$,
$139(16), 131(32)[\mathrm{PhCCCHOH}]^{+}, 129(24)$
$[\mathrm{PhCCCO}]^{+}, 115(39)\left[\mathrm{PhCCCH}_{2}\right]^{+}, 102(28)$
$[\mathrm{PhCCH}]^{+}, 77(22)\left[\mathrm{C}_{6} \mathrm{H}_{5}\right]^{+}, 45(48)[\mathrm{MOM}]^{+}$.
$\widetilde{v}\left(\mathrm{~cm}^{-1}\right)=3354(\mathrm{~s}), 2935(\mathrm{~m}), 2233(\mathrm{w})$,
$1600(\mathrm{w}), 1571(\mathrm{w}), 1549(\mathrm{w}), 1490(\mathrm{~s}), 1464(\mathrm{w})$,
$1442(\mathrm{~m}), 1398(\mathrm{~m}), 1381(\mathrm{~m}), 1298(\mathrm{~m})$,
$1251(\mathrm{~m}), 1191(\mathrm{w}), 1148(\mathrm{~m}), 1133(\mathrm{~m})$,
$1095(\mathrm{~s}), 1071(\mathrm{w}), 1033(\mathrm{~s}), 1020(\mathrm{~s}), 998(\mathrm{~m})$,
$964(\mathrm{~m}), 916(\mathrm{~m}), 842(\mathrm{~m}), 815(\mathrm{~m}), 757(\mathrm{vs})$,
$691(\mathrm{vs}), 581(\mathrm{w}), 528(\mathrm{w})$.

\subsubsection{1-(Methoxymethyl)-3,5-bis(1-chloro-3-phenylprop-2-yn-2-yl)-1H- pyrazole}

(21)

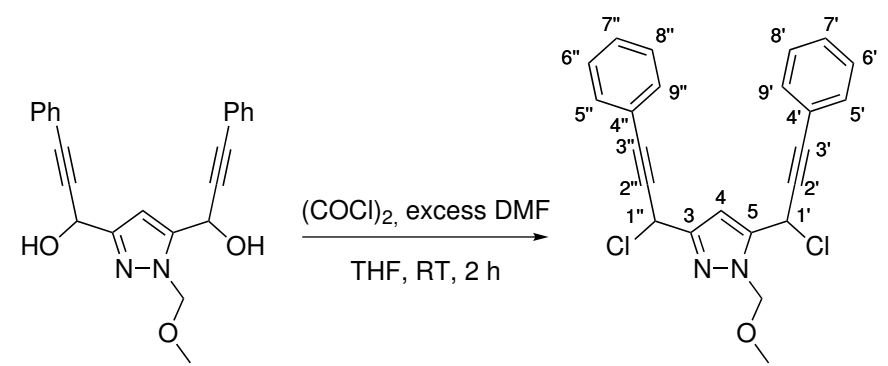

Oxalylchloride (1.05 mL, $1.525 \mathrm{~g}, 12.02 \mathrm{mmol}, 2.7 \mathrm{eq}$.) was added to a solution of DMF (34.5 mL, $32.8 \mathrm{~g}, 448.4 \mathrm{mmol}, 101 \mathrm{eq}$ ) ) and acetonitrile $(83 \mathrm{~mL})$ at $-20{ }^{\circ} \mathrm{C}$ under inert conditions and stirred for $20 \mathrm{~min}$ at this temperature. Subsequently 1-(methoxymethyl)3,5-bis(1-hydroxy-3-phenylprop-2-yn-2-yl)-1 $H$-pyrazole (20) (1.66 g, $4.45 \mathrm{mmol}, 1.0 \mathrm{eq}$.) in $\mathrm{MeCN}(80 \mathrm{~mL})$ was added dropwise and the reaction mixture was stirred for $20 \mathrm{~min}$ at $-20{ }^{\circ} \mathrm{C}$ and $2.5 \mathrm{~h}$ at ambient temperature. The solvent was removed under reduced pressure and the raw product was purified by column chromatography (silica, $n$-hexane/EtOAc $\left.2: 1, \mathrm{R}_{f}=0.61\right)$. The product was obtained as a yellow solid (1.24 g, $68 \%)$.
Empirical Formula:
$\mathrm{C}_{23} \mathrm{H}_{18} \mathrm{~N}_{2} \mathrm{Cl}_{2} \mathrm{O}$
Molecular Weight $(\mathrm{g} / \mathrm{mol})$ :
409.31 
${ }^{\mathbf{1}} \mathbf{H}$ NMR $\left(500 \mathrm{MHz}\right.$, acetone- $\left.\mathrm{d}_{6}\right): \quad \delta / \mathrm{ppm}=3.38\left(\mathrm{~s}, 3 \mathrm{H}, \mathrm{CH}_{3}\right), 5.62(\mathrm{~d}, 1 \mathrm{H}$, $\left.{ }^{3} J(\mathrm{H}, \mathrm{H})=10 \mathrm{~Hz}, \quad \mathrm{CH}_{2}\right), \quad 5.70 \quad(\mathrm{~d}, \quad 1 \mathrm{H}$, $\left.{ }^{3} J(\mathrm{H}, \mathrm{H})=10 \mathrm{~Hz}, \mathrm{CH}_{2}\right), 6.24(\mathrm{~s}, 1 \mathrm{H}, 1$ '-H), 6.51 (s, 1H, 1'-H), 7.05 (s, 1H, $\left.\mathrm{H}^{\mathrm{pz}}\right), 7.38-7.48(\mathrm{~m}, 6 \mathrm{H}$, 6'-H, 7'-H, 8'-H, 6"-H, 7'-H, 8'-H), 7.51-7.54 (m,

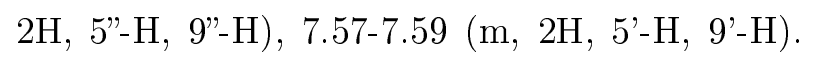
(All signals are doubled due to the presence of diastereomeric pairs of enantiomers.)

${ }^{13}$ C NMR (125 MHz, acetone- $\left.\mathrm{d}_{6}\right)$ : $\delta / \mathrm{ppm}=40.9\left(\mathrm{C} 1^{\prime}\right), 44.6(\mathrm{C} 1 ”), 57.0\left(\mathrm{CH}_{3}\right), 81.3$ $\left(\mathrm{CH}_{2}\right), 84.7$ (C2'), 86.3 (C2"), 88.0 (C3"), 88.5 (C3'), 107.5 (C4), 122.2 (C4'), 122.6 (C4”), 130.2 (C6", C8”), 130.5 (C6', C8'), 129.5 (C7'/C7”), 129.7 (C7'/C7”), 132.5 (C5”, C9”), 132.7 (C5', C9'), 142.3 (C5), 150.4 (C3).

MS (EI): $m / z=$ $409(5)[\mathrm{M}]^{+}, 373(100)[\mathrm{M}-\mathrm{HCl}]^{+}, 338(13)[\mathrm{M}-$ $\mathrm{HCl}-\mathrm{Cl}^{+}, 328$ (24) $[\mathrm{M}-\mathrm{HCl}-\mathrm{MOM}]^{+}, 308$ (24) $[\mathrm{M}-\mathrm{HCl}-\mathrm{Cl}-\mathrm{MOM}+\mathrm{OH}]^{+}, 265$ (56), 259 (17), 139 (37), 115 (18) $\left[\mathrm{PhCCCH}_{2}\right]^{+}, 73$ (38), 45 (98) $[\mathrm{MOM}]^{+}, 36(95)[\mathrm{HCl}]^{+}$.

IR $(\mathrm{KBr})$ : $\widetilde{v}\left(\mathrm{~cm}^{-1}\right)=3134(\mathrm{w}), 3079(\mathrm{w}), 3057(\mathrm{w})$, $3029(\mathrm{w}), 3021(\mathrm{w}), 2997(\mathrm{w}), 2935(\mathrm{~m})$, $2849(\mathrm{w}), 2248(\mathrm{w}), 2195(\mathrm{w}), 1946(\mathrm{w})$, $1885(\mathrm{w}), 1808$ (w), 1730 (m), 1644 (vs), 1598 (w), 1557 (w), 1490 (s), 1443 (s), 1430 (s), $1362(\mathrm{~s}), 1288(\mathrm{w}), 1251(\mathrm{~m}), 1191(\mathrm{w}), 1153(\mathrm{w})$, $1095(\mathrm{~m}), 1029(\mathrm{w}), 966(\mathrm{w}), 914(\mathrm{~m}), 814(\mathrm{~m})$, 758 (s), 691 (s), 531 (m), $440(\mathrm{~m})$.

\subsubsection{3,5-Bis(1-hydroxy-3-phenylprop-2-yn-2-yl)-1H-pyrazole (18)}
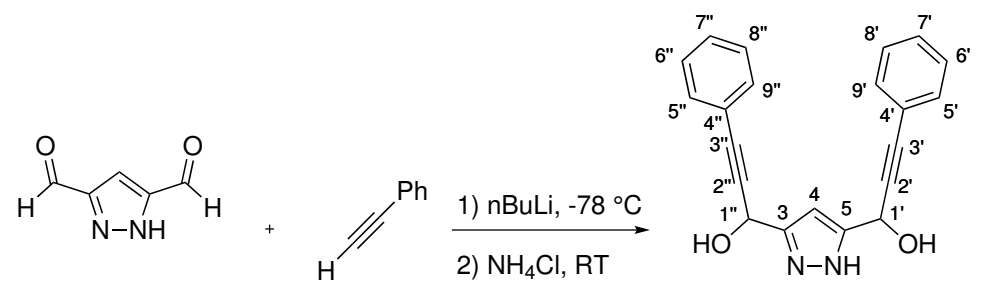

$1 H$-pyrazole-3,5-dicarbaldehyde (16) (508 $\mathrm{mg}, 4,09 \mathrm{mmol}, 1.0$ eq.) was suspended in THF (300 mL) in an ultrasonic bath connected to water cooling over night. $n \operatorname{BuLi}(2.5 \mathrm{M}, 6.55 \mathrm{~mL}, 16.4 \mathrm{mmol}, 4.0 \mathrm{eq}$.) was added to a solution of Phenylacetylene $(1.79 \mathrm{~mL}, 1.67 \mathrm{~g}, 16.4 \mathrm{mmol}, 4.0$ eq. $)$ in dry THF $(75 \mathrm{~mL})$ at $-78{ }^{\circ} \mathrm{C}$ under inert conditions and the mixture was stirred for $1 \mathrm{~h}$. Subsequently the mixture was added 
dropwise to the suspension of $1 H$-pyrazole-3,5-dicarbaldehyde (16) $(1.61 \mathrm{~g}, 9.95 \mathrm{mmol}$, 1.0 eq.) in THF. The mixture was treated in the ultrasonic bath for additional $5 \mathrm{~h}$. After addition of $300 \mathrm{~mL}$ of a saturaed aqueous solution of $\mathrm{NH}_{4} \mathrm{Cl}$ the mixture was extracted with THF $(3 \times 50 \mathrm{~mL})$, washed with brine $(100 \mathrm{~mL})$ and dried over sodium sulfate. The solvent was removed under reduced pressure and the raw product was purified by column chromatography (silica, $n$-hexane/EtOAc $1: 3, \mathrm{R}_{f}=0.43$ ). The product was obtained as a yellow solid (343 mg, $26 \%$ ).

\begin{tabular}{|c|c|}
\hline Empirical Formula: & $\mathrm{C}_{21} \mathrm{H}_{16} \mathrm{~N}_{2} \mathrm{O}_{2}$ \\
\hline Molecular Weight (g/mol): & 328.36 \\
\hline${ }^{\mathbf{1}} \mathbf{H}$ NMR (400 MHz, acetone- $\left.\mathrm{d}_{6}\right)$ : & $\begin{array}{l}\delta / \mathrm{ppm}=5.77 \quad\left(\mathrm{~s}, \quad 2 \mathrm{H}, \quad 1^{\prime}-\mathrm{H}\right), \quad 6.57 \quad(\mathrm{~d}, \\
\left.{ }^{3} J(\mathrm{H}, \mathrm{H})=2.5 \mathrm{~Hz}, 1 \mathrm{H}, \mathrm{H}^{\mathrm{pz}}\right), \quad 7.34-7.36(\mathrm{~m}, \\
\left.6 \mathrm{H}, 6{ }^{\prime}-\mathrm{H}, 7^{\prime}-\mathrm{H}, 8^{\prime}-\mathrm{H}\right), 7.43-7.48\left(\mathrm{~m}, 4 \mathrm{H}, 5^{\prime}-\mathrm{H},\right. \\
\left.9^{\prime}-\mathrm{H}\right), 12.14(\mathrm{~s} \mathrm{br}, 1 \mathrm{H}, \mathrm{NH}) .\end{array}$ \\
\hline${ }^{13} \mathbf{C}$ NMR (100 MHz, acetone- $\left.\mathrm{d}_{6}\right)$ : & $\begin{array}{l}\delta / \mathrm{ppm}=58.9\left(\mathrm{C}^{\prime}\right), 85.0\left(\mathrm{C} 3^{\prime}\right), 90.2\left(\mathrm{C}^{\prime}\right), 102.0 \\
(\mathrm{C} 4), 123.7\left(\mathrm{C} 4^{\prime}\right), 129.3\left(\mathrm{C} 6 ', \mathrm{C} 8^{\prime}\right), 129.8\left(\mathrm{C} 7^{\prime}\right), \\
132.4\left(\mathrm{C} 5{ }^{\prime}, \mathrm{C}^{\prime}\right), 149.6(\mathrm{C} 3, \mathrm{C} 5) .\end{array}$ \\
\hline IR $(\mathrm{KBr})$ : & $\begin{array}{l}\widetilde{v}\left(\mathrm{~cm}^{-1}\right)=3140(\mathrm{w}), 3079(\mathrm{w}), 3059(\mathrm{w}), \\
3029(\mathrm{w}), 3021(\mathrm{w}), 2997(\mathrm{w}), 2961(\mathrm{w}), \\
2925(\mathrm{w}), 2873(\mathrm{~m}), 2361(\mathrm{w}), 2338(\mathrm{w}), \\
2231(\mathrm{~m}), 2196(\mathrm{~s}), 1958(\mathrm{w}), 1884(\mathrm{w}), 1808(\mathrm{w}), \\
1692(\mathrm{~s}), 1636(\mathrm{~s}), 1597(\mathrm{~m}), 1570(\mathrm{~m}), 1490(\mathrm{vs}), \\
1443(\mathrm{~s}), 1402(\mathrm{~m}), 1261(\mathrm{~s}), 1153(\mathrm{~s}), 1133(\mathrm{~s}), \\
1032(\mathrm{~s}), 1016(\mathrm{~s}), 997(\mathrm{~s}), 956(\mathrm{~s}), 917(\mathrm{w}), \\
829(\mathrm{~m}), 801(\mathrm{~m}), 755(\mathrm{vs}), 690(\mathrm{vs}), 584(\mathrm{~m}), \\
532(\mathrm{~m}) .\end{array}$ \\
\hline
\end{tabular}

\subsubsection{3,5-Bis(1-chloro-3-phenylprop-2-yn-2-yl)-1H-pyrazole (22)}
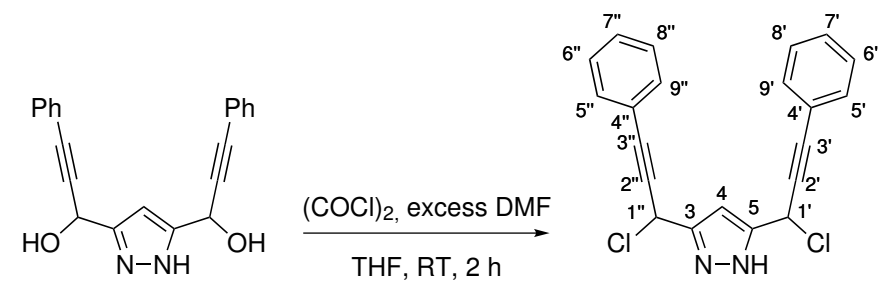

Oxalylchloride (0.186 mL, $270 \mathrm{mg}, 2.13 \mathrm{mmol}, 2.7$ eq.) was added to a solution of DMF $\left(6.5 \mathrm{~mL}, 6.18 \mathrm{~g}, 84.5 \mathrm{mmol}, 107 \mathrm{eq}\right.$ ) and acetonitrile $(20 \mathrm{~mL})$ at $-20{ }^{\circ} \mathrm{C}$ under inert conditions and stirred for 20 min at this temperature. Subsequently 3,5-bis(1-hydroxy-3phenylprop-2-yn-2-yl)- $1 H$-pyrazole (18) (260 mg, $0.792 \mathrm{mmol}, 1.0$ eq.) in $\mathrm{MeCN}$ (20 mL) was added dropwise and the reaction mixture was stirred for $30 \mathrm{~min}$ at $-20{ }^{\circ} \mathrm{C}$ and $3.5 \mathrm{~h}$ 
at ambient temperature. The solvent was removed under reduced pressure and the raw product was obtained as a yellow brownish solid (100 mg, $35 \%$ ).

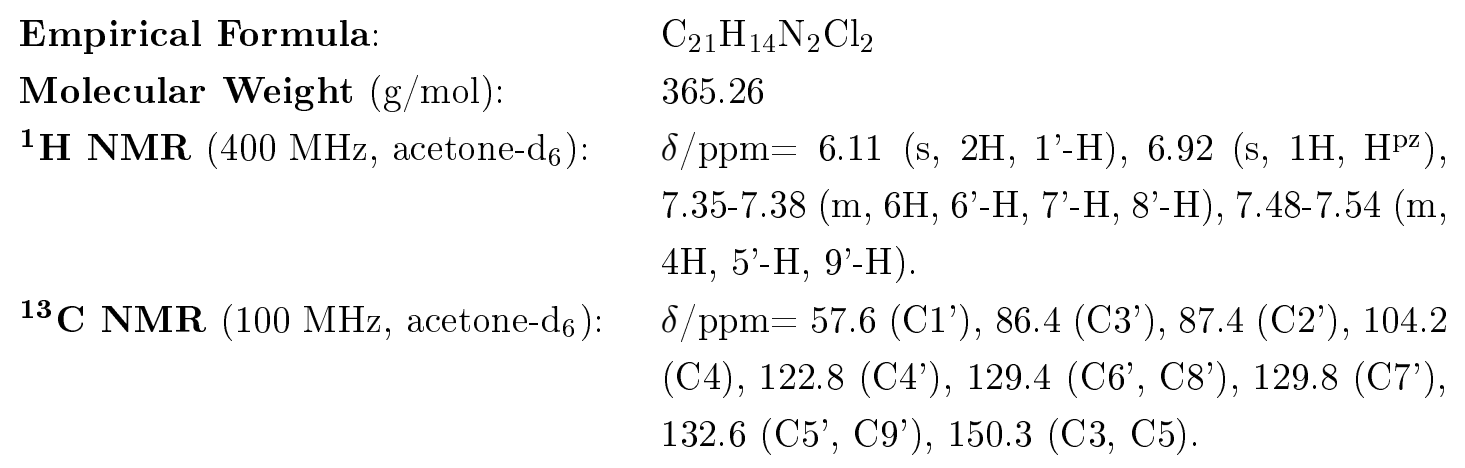

\subsection{Synthesis of Siamese-Twin Porphyrins ${ }^{\mathrm{X}} \mathrm{LH}_{4}$}

\subsubsection{General Procedure for the Synthesis of a Siamese-Twin Porphyrinogen ${ }^{\mathrm{X}} \mathrm{LH}_{6}$}
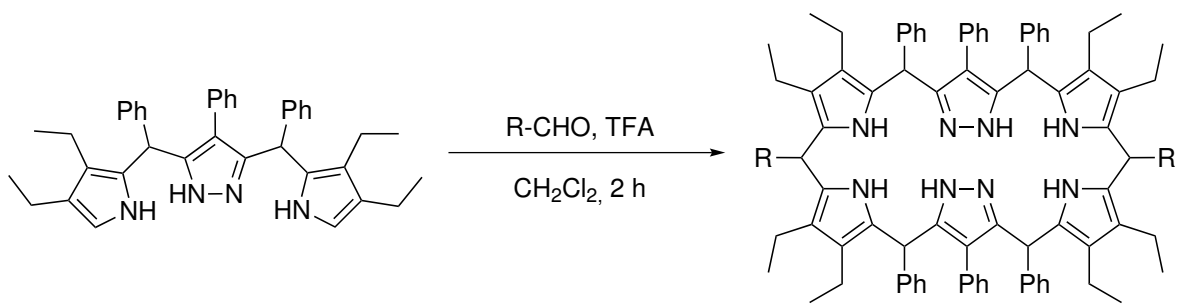

The aldehyde (0.180 mmol, 1.0 eq.) and 3,5-Bis-(3,4-diethyl- $H$-pyrrole-2-yl-benzyl)$1 \mathrm{H}$-pyrazole (13) (0.180 mmol, 1.0 eq.) were dissolved in dichloromethane $(18.6 \mathrm{~mL})$ and protected from light. TFA $(180 \mu \mathrm{L}, 1 \mathrm{M}$ in DCM, $20.5 \mathrm{mg}, 0.180 \mathrm{mmol}, 1.0$ eq.) was added and the solution was stirred for $2 \mathrm{~h}$. The solvent was reduced to less than $5 \%$ and the solution was filtered over a plug of basic aluminum oxide. The only fraction passing was the product. The Siamese-twin porphyrinogen ${ }^{\mathbf{X}} \mathbf{L H}_{\mathbf{6}}$ was obtained as a yellow solid and directly used for the oxidation to the corresponding Siamese-twin porphyrin ${ }^{\mathbf{X}_{\mathbf{L}}} \mathbf{L H}_{\mathbf{4}}$.

${ }^{\mathrm{pMe}} \mathrm{LH}_{6}$

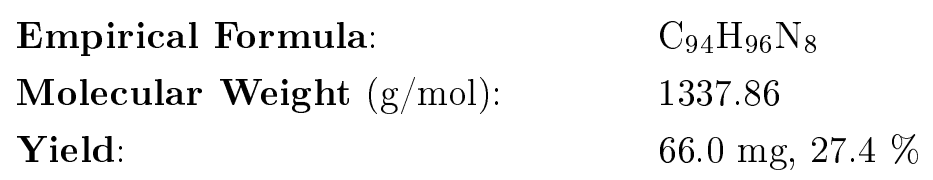

${ }^{\mathrm{pF}} \mathrm{LH}_{6}$

Empirical Formula: $\quad \mathrm{C}_{92} \mathrm{H}_{90} \mathrm{~N}_{8} \mathrm{~F}_{2}$

Molecular Weight $(\mathrm{g} / \mathrm{mol}): \quad 1345.78$ 
Yield:

$64.8 \mathrm{mg}, 26.7 \%$

${ }^{\mathrm{MeO}} \mathrm{LH}_{6}$

Empirical Formula:

Molecular Weight $(\mathrm{g} / \mathrm{mol})$ :

Yield:

${ }^{\mathrm{pMeO}} \mathrm{LH}_{6}$

Empirical Formula:

Molecular Weight $(\mathrm{g} / \mathrm{mol})$ :

Yield:
$\mathrm{C}_{98} \mathrm{H}_{104} \mathrm{~N}_{8} \mathrm{O}_{6}$

1489.96

$46.5 \mathrm{mg}, 17.3 \%$

\subsubsection{General Procedure for the Synthesis of Siamese-Twin Porphyrins $\mathrm{X}_{\mathrm{LH}_{4}}$}
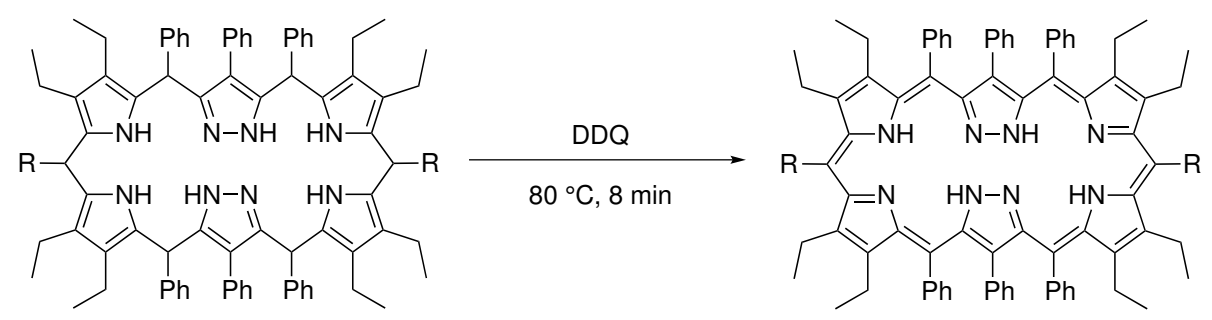

The Siamese-twin porphyrinogen ${ }^{\mathbf{X}} \mathbf{L H}_{\mathbf{6}}$ (0.310 mmol, 1.0 eq.) was dissolved in toluene $(60.0 \mathrm{~mL})$ at $80{ }^{\circ} \mathrm{C}$, DDQ $(1.24 \mathrm{mmol}, 4.0$ eq. $)$ was added all at once and the solution was stirred for $8 \mathrm{~min}$ at $80{ }^{\circ} \mathrm{C}$. The solvent was immediately removed under reduced pressure and the residue was dissolved in a mixture of methyl tert-butyl ether/dichloromethane/EtOAc (10:3:1) and filtered over a plug of basic aluminum oxide. The only fraction passing was the raw product which was further purified by column chromatography (silica, MeOH). The Siamese-twin porphyrin ${ }^{\mathbf{X}} \mathbf{L H}_{\mathbf{4}}$ was obtained as a green blueish solid.

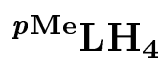

Crystals suitable for X-Ray rystallography were obtained by cooling down a nearly saturated solution of ${ }^{p \mathrm{Me}} \mathbf{L H}_{4}$ in dichloromethane to $-35{ }^{\circ} \mathrm{C}$.

$\begin{array}{ll}\text { Empirical Formula: } & \mathrm{C}_{94} \mathrm{H}_{88} \mathrm{~N}_{8} \\ \text { Molecular Weight }(\mathrm{g} / \mathrm{mol}): & 1329.79 \\ \text { Yield: } & 98.5 \mathrm{mg}, 23.9 \% \\ \mathbf{R}_{f} \text { (silica, MeOH): } & 0.30\end{array}$


HRMS $\left(\mathrm{ESI}^{+}, \mathrm{MeOH}\right): m / z=$

IR $(\mathrm{KBr})$ :
1329.7217 (calcd. 1329.7205) $[\mathrm{M}+\mathrm{H}]^{+}, 665.3634$ (calcd. 665.3639) $[\mathrm{M}+2 \mathrm{H}]^{2+}$.

$\widetilde{v}\left(\mathrm{~cm}^{-1}\right)=3074(\mathrm{w}), 3055(\mathrm{w}), 3022(\mathrm{w})$, 2972 (vs), 2930 (s), 2895 (w), 2871 (m), 1645 (s), $1604(\mathrm{~m}), 1574(\mathrm{w}), 1525(\mathrm{vs}), 1506$ (s), $1494(\mathrm{~m}), 1454$ (m), 1442 (m), 1392 (m), 1360 (vs), 1313 (m), 1297 (w), 1251 (m), $1208(\mathrm{~m}), 1180(\mathrm{~s}), 1136(\mathrm{~s}), 1117(\mathrm{~m}), 1107(\mathrm{w})$, $1052(\mathrm{~m}), 1011(\mathrm{~m}), 950(\mathrm{w}), 922(\mathrm{w}), 905(\mathrm{w})$, $875(\mathrm{w}), 814(\mathrm{w}), 755(\mathrm{w}), 697(\mathrm{~s}), 668(\mathrm{~m})$, $547(\mathrm{w}), 515(\mathrm{w}), 397(\mathrm{w})$.

UV-vis $/ \mathbf{n m}\left(\epsilon / \mathrm{M}^{-1} \mathrm{~cm}^{-1}\right)$ :
278 (30600), 307 (32700), 390 (89000), 640 (37800), 734 (13500).

${ }^{1} \mathbf{H}$ NMR $\left(500 \mathrm{MHz}, \mathrm{CD}_{2} \mathrm{Cl}_{2}, 238 \mathrm{~K}\right): \delta / \mathrm{ppm}=0.41\left(\mathrm{t},{ }^{3} J(\mathrm{H}-\mathrm{H})=7.3 \mathrm{~Hz}, 3 \mathrm{H}\right.$, $\left.\mathrm{CH}_{3}\right), 0.45\left(\mathrm{t},{ }^{3} \mathrm{~J}(\mathrm{H}-\mathrm{H})=7.3 \mathrm{~Hz}, 3 \mathrm{H}, \mathrm{CH}_{3}\right)$, $0.55\left(\mathrm{t},{ }^{3} J(\mathrm{H}-\mathrm{H})=7.3 \mathrm{~Hz}, 6 \mathrm{H}, \mathrm{CH}_{3}\right), 0.89$ $\left(\mathrm{q},{ }^{3} \mathrm{~J}(\mathrm{H}-\mathrm{H})=7.3 \mathrm{~Hz}, 1 \mathrm{H}, \mathrm{CH}_{2}\right), 1.20$ (q, $\left.{ }^{3} J(\mathrm{H}-\mathrm{H})=7.3 \mathrm{~Hz}, 1 \mathrm{H}, \mathrm{CH}_{2}\right), 1.43\left(\mathrm{q},{ }^{3} J(\mathrm{H}-\right.$ $\left.\mathrm{H})=7.3 \mathrm{~Hz}, 2 \mathrm{H}, \mathrm{CH}_{2}\right), \quad 1.55\left(\mathrm{q},{ }^{3} J(\mathrm{H}-\right.$ $\left.\mathrm{H})=7.3 \mathrm{~Hz}, 2 \mathrm{H}, \mathrm{CH}_{2}\right), 1.86\left(\mathrm{q},{ }^{3} J(\mathrm{H}-\right.$ $\left.\mathrm{H})=7.3 \mathrm{~Hz}, 2 \mathrm{H}, \mathrm{CH}_{2}\right), 1.98\left(\mathrm{q},{ }^{3} J(\mathrm{H}-\right.$ $\left.\mathrm{H})=7.3 \mathrm{~Hz}, 2 \mathrm{H}, \mathrm{CH}_{2}\right), 2.32\left(\mathrm{~s}, 3 \mathrm{H}, \mathrm{CH}_{3}\right)$, $6.17\left(\mathrm{~d},{ }^{3} J(\mathrm{H}-\mathrm{H})=7.7 \mathrm{~Hz}, 1 \mathrm{H}, \mathrm{Ph}\right), 6.29(\mathrm{td}$, $\left.{ }^{4} J(\mathrm{H}-\mathrm{H})=1.3 \mathrm{~Hz},{ }^{3} J(\mathrm{H}-\mathrm{H})=7.5 \mathrm{~Hz}, 1 \mathrm{H}, \mathrm{Ph}\right)$, $6.55\left(\mathrm{td},{ }^{4} J(\mathrm{H}-\mathrm{H})=1.3 \mathrm{~Hz},{ }^{3} J(\mathrm{H}-\mathrm{H})=7.5 \mathrm{~Hz}\right.$, $1 \mathrm{H}, \mathrm{Ph}), 6.74\left(\mathrm{td},{ }^{4} J(\mathrm{H}-\mathrm{H})=1.5 \mathrm{~Hz},{ }^{3} J(\mathrm{H}-\right.$ $\mathrm{H})=7.5 \mathrm{~Hz}, 1 \mathrm{H}, \mathrm{Ph}), 6.79\left(\mathrm{td},{ }^{4} J(\mathrm{H}-\right.$ $\left.\mathrm{H})=1.5 \mathrm{~Hz},{ }^{3} J(\mathrm{H}-\mathrm{H})=7.5 \mathrm{~Hz}, 1 \mathrm{H}, \mathrm{Ph}\right)$, $6.84\left(\mathrm{~d},{ }^{3} J(\mathrm{H}-\mathrm{H})=7.7 \mathrm{~Hz}, 1 \mathrm{H}, \mathrm{Ph}\right), 6.92(\mathrm{~d}$, $\left.{ }^{3} J(\mathrm{H}-\mathrm{H})=7.7 \mathrm{~Hz}, 1 \mathrm{H}, \mathrm{Ph}\right), 6.97\left(\mathrm{td},{ }^{4} J(\mathrm{H}-\right.$ $\left.\mathrm{H})=1.3 \mathrm{~Hz},{ }^{3} J(\mathrm{H}-\mathrm{H})=7.5 \mathrm{~Hz}, 1 \mathrm{H}, \mathrm{Ph}\right), 7.23-$ $7.34(\mathrm{~m}, 5 \mathrm{H}, \mathrm{Ph}), 7.54(\mathrm{~m}, 2 \mathrm{H}, \mathrm{Ph}), 11.32(\mathrm{~s}$, $\left.1 \mathrm{H}, \mathrm{NH}^{\mathrm{pyr}}\right), 13.36$ (s, 1H, NH $\left.{ }^{\mathrm{pz}}\right)$. 
${ }^{13} \mathrm{C}$ NMR $\left(125 \mathrm{MHz}, \mathrm{CD}_{2} \mathrm{Cl}_{2}, 238 \mathrm{~K}\right): \delta / \mathrm{ppm}=14.7\left(\mathrm{CH}_{3}\right), 15.6\left(\mathrm{CH}_{3}\right), 15.9\left(\mathrm{CH}_{3}\right)$, $16.3\left(\mathrm{CH}_{3}\right), 18.0\left(\mathrm{CH}_{2}\right), 18.1\left(\mathrm{CH}_{2}\right), 18.5\left(\mathrm{CH}_{2}\right)$, $18.9\left(\mathrm{CH}_{2}\right), 21.4\left(\mathrm{CH}_{3}\right), 106.0(\mathrm{Ph}), 113.8(\mathrm{Ph})$, $123.9(\mathrm{Ph}), 124.2(\mathrm{Ph}), 124.4(\mathrm{Ph}), 126.1(\mathrm{Ph})$, $126.5(\mathrm{Ph}), 126.5(\mathrm{Ph}), 127.0(\mathrm{Ph}), 127.2(\mathrm{Ph})$, $127.2(\mathrm{Ph}), 127.6(\mathrm{Ph}), 127.7(\mathrm{Ph}), 127.7(\mathrm{Ph})$, $127.8(\mathrm{Ph}), 127.9(\mathrm{Ph}), 128.3(\mathrm{Ph}), 130.7(\mathrm{Ph})$, $131.6(\mathrm{Ph}), 132.3(\mathrm{Ph}), 132.5(\mathrm{Ph}), 132.9(\mathrm{Ph})$, $133.1(\mathrm{Ph}), 133.9(\mathrm{Ph}), 134.8(\mathrm{Ph}), 135.3(\mathrm{Ph})$, $137.4(\mathrm{Ph}), 139.1(\mathrm{Ph}), 139.4(\mathrm{Ph}), 140.7(\mathrm{Ph})$, $141.3(\mathrm{Ph}), 141.4(\mathrm{Ph}), 145.3(\mathrm{Ph}), 147.5(\mathrm{Ph})$, $148.2(\mathrm{Ph}), 151.2(\mathrm{Ph}), 151.4(\mathrm{Ph}), 167.7(\mathrm{Ph})$.

\section{${ }^{p \mathrm{~F}} \mathrm{LH}_{4}$}

Crystals suitable for X-Ray crystallography were obtained by cooling down a nearly saturated solution of ${ }^{\mathbf{p}} \mathbf{L H}_{4}$ in dichloromethane to $-35{ }^{\circ} \mathrm{C}$.

Empirical Formula:

Molecular Weight $(\mathrm{g} / \mathrm{mol})$ :

Yield:

$\mathbf{R}_{f}$ (silica, $\mathrm{MeOH}$ ):

HRMS $\left(\mathrm{ESI}^{+}, \mathrm{MeOH}\right): m / z=$

IR $(\mathrm{KBr})$ :

UV-vis $/ \mathbf{n m}\left(\epsilon / \mathrm{M}^{-1} \mathrm{~cm}^{-1}\right)$ :
$\mathrm{C}_{92} \mathrm{H}_{82} \mathrm{~N}_{8} \mathrm{~F}_{2}$

1337.72

$37.2 \mathrm{mg}, 9.0 \%$

0.50

1337.6704 (calcd. 1337.6703) $[\mathrm{M}+\mathrm{H}]^{+}, 669.3384$ (calcd. 669.3388) $[\mathrm{M}+2 \mathrm{H}]^{2+}$.

$\widetilde{v}\left(\mathrm{~cm}^{-1}\right)=3074(\mathrm{w}), 3055(\mathrm{w}), 3022(\mathrm{w})$, 2968 (s), 2928 (s), 2896 (w), $2870(\mathrm{~m}), 1640(\mathrm{~m})$, 1633 (m), 1600 (m), 1569 (m), 1521 (vs), 1505 (vs), 1497 (s), 1471 (m), 1442 (m), 1387 (s), 1362 (vs), 1313 (m), 1257 (w), 1222 (s), $1186(\mathrm{~m}), 1154$ (s), 1136 (s), 1118 (s), 1073 (w), $1052(\mathrm{w}), 1016(\mathrm{~m}), 955(\mathrm{w}), 825(\mathrm{~m}), 811(\mathrm{~m})$, $763(\mathrm{~m}), 698(\mathrm{vs}), 674(\mathrm{w}), 583(\mathrm{w}), 544(\mathrm{w})$, $343(\mathrm{w})$.

306 (30900), 389 (83200), 637 (36900), 734 (12700). 
${ }^{1} \mathbf{H}$ NMR $\left(500 \mathrm{MHz}, \mathrm{CD}_{2} \mathrm{Cl}_{2}, 238 \mathrm{~K}\right): \delta / \mathrm{ppm}=0.42\left(\mathrm{t},{ }^{3} J(\mathrm{H}-\mathrm{H})=7.3 \mathrm{~Hz}, 3 \mathrm{H}\right.$, $\left.\mathrm{CH}_{3}\right), 0.45\left(\mathrm{t},{ }^{3} J(\mathrm{H}-\mathrm{H})=7.3 \mathrm{~Hz}, 3 \mathrm{H}, \mathrm{CH}_{3}\right)$, $0.56\left(\mathrm{t},{ }^{3} \mathrm{~J}(\mathrm{H}-\mathrm{H})=7.3 \mathrm{~Hz}, 6 \mathrm{H}, \mathrm{CH}_{3}\right), 0.90$ $\left(\mathrm{q},{ }^{3} J(\mathrm{H}-\mathrm{H})=7.3 \mathrm{~Hz}, 1 \mathrm{H}, \mathrm{CH}_{2}\right), 1.20(\mathrm{q}$, $\left.{ }^{3} J(\mathrm{H}-\mathrm{H})=7.3 \mathrm{~Hz}, 1 \mathrm{H}, \mathrm{CH}_{2}\right), 1.45\left(\mathrm{q},{ }^{3} J(\mathrm{H}-\right.$ $\left.\mathrm{H})=7.3 \mathrm{~Hz}, 2 \mathrm{H}, \mathrm{CH}_{2}\right), 1.57\left(\mathrm{q},{ }^{3} J(\mathrm{H}-\right.$ $\left.\mathrm{H})=7.3 \mathrm{~Hz}, 2 \mathrm{H}, \mathrm{CH}_{2}\right), 1.86\left(\mathrm{q},{ }^{3} J(\mathrm{H}-\right.$ $\left.\mathrm{H})=7.3 \mathrm{~Hz}, 1 \mathrm{H}, \mathrm{CH}_{2}\right), 1.98\left(\mathrm{q},{ }^{3} J(\mathrm{H}-\right.$ $\left.\mathrm{H})=7.3 \mathrm{~Hz}, 1 \mathrm{H}, \mathrm{CH}_{2}\right), 6.17\left(\mathrm{dd},{ }^{4} J(\mathrm{H}-\right.$ $\left.\mathrm{H})=2.0 \mathrm{~Hz},{ }^{3} J(\mathrm{H}-\mathrm{H})=7.5 \mathrm{~Hz}, 1 \mathrm{H}, \mathrm{Ph}\right), 6.29$ $\left(\mathrm{td},{ }^{4} J(\mathrm{H}-\mathrm{H})=1.3 \mathrm{~Hz},{ }^{3} J(\mathrm{H}-\mathrm{H})=7.5 \mathrm{~Hz}\right.$, $1 \mathrm{H}, \mathrm{Ph}), 6.56\left(\mathrm{td},{ }^{4} J(\mathrm{H}-\mathrm{H})=1.3 \mathrm{~Hz},{ }^{3} J(\mathrm{H}-\right.$ $\mathrm{H})=7.5 \mathrm{~Hz}, 1 \mathrm{H}, \mathrm{Ph}), 6.75\left(\mathrm{td},{ }^{4} J(\mathrm{H}-\right.$ $\left.\mathrm{H})=1.5 \mathrm{~Hz},{ }^{3} J(\mathrm{H}-\mathrm{H})=7.5 \mathrm{~Hz}, 1 \mathrm{H}, \mathrm{Ph}\right), 6.79$ $\left(\mathrm{td},{ }^{4} J(\mathrm{H}-\mathrm{H})=1.5 \mathrm{~Hz},{ }^{3} J(\mathrm{H}-\mathrm{H})=7.5 \mathrm{~Hz}\right.$, $1 \mathrm{H}, \mathrm{Ph}), 6.83-6.86(\mathrm{~m}, 1 \mathrm{H}, \mathrm{Ph}), 6.89-7.05(\mathrm{~m}$, $5 \mathrm{H}, \mathrm{Ph}), 7.13-7.17(\mathrm{~m}, 1 \mathrm{H}, \mathrm{Ph}), 7.21-7.38(\mathrm{~m}$, $5 \mathrm{H}, \mathrm{Ph}), 7.48-7.55(\mathrm{~m}, 2 \mathrm{H}, \mathrm{Ph}), 11.37(\mathrm{~s}, 1 \mathrm{H}$, $\left.\mathrm{NH}^{\mathrm{pyr}}\right), 13.34\left(\mathrm{~s}, 1 \mathrm{H}, \mathrm{NH}^{\mathrm{pz}}\right)$.

${ }^{13} \mathrm{C}$ NMR $\left(125 \mathrm{MHz}, \mathrm{CD}_{2} \mathrm{Cl}_{2}, 238 \mathrm{~K}\right): \delta / \mathrm{ppm}=14.8\left(\mathrm{CH}_{3}\right), 15.5\left(\mathrm{CH}_{3}\right), 15.9\left(\mathrm{CH}_{3}\right)$, $16.2\left(\mathrm{CH}_{3}\right), \quad 18.1\left(\mathrm{CH}_{2}\right), \quad 18.2\left(\mathrm{CH}_{2}\right), \quad 18.5$ $\left(\mathrm{CH}_{2}\right), 18.9\left(\mathrm{CH}_{2}\right), 104.6(\mathrm{Ph}), 114.1\left(\mathrm{~d},{ }^{2} J(\mathrm{C}-\right.$ $\mathrm{F})=21.0 \mathrm{~Hz}, \mathrm{Ph}), 114.4(\mathrm{Ph}), 114.6\left(\mathrm{~d},{ }^{2} J(\mathrm{C}-\right.$ $\mathrm{F})=21.0 \mathrm{~Hz}, \mathrm{Ph}), 124.0(\mathrm{Ph}), 124.4(\mathrm{Ph}), 126.2$ $(\mathrm{Ph}), 126.5(\mathrm{Ph}), 126.6(\mathrm{Ph}), 127.1(\mathrm{Ph}), 127.2$ $(\mathrm{Ph}), 127.3(\mathrm{Ph}), 127.7(\mathrm{Ph}), 127.8(\mathrm{Ph}), 128.0$ $(\mathrm{Ph}), 131.6(\mathrm{Ph}), 131.6\left(\mathrm{~d},{ }^{1} \mathrm{~J}(\mathrm{C}-\mathrm{F})=227.7 \mathrm{~Hz}\right.$, $\mathrm{Ph}), 132.3(\mathrm{Ph}), 133.1(\mathrm{Ph}), 133.8(\mathrm{Ph}), 134.1$ (Ph), $134.1(\mathrm{Ph}), 134.2\left(\mathrm{~d},{ }^{3} J(\mathrm{C}-\mathrm{F})=7.9 \mathrm{~Hz}\right.$ $\mathrm{Ph}), 134.7\left(\mathrm{~d},{ }^{3} J(\mathrm{C}-\mathrm{F})=7.9 \mathrm{~Hz}, \mathrm{Ph}\right), 135.2$ (Ph), $139.1(\mathrm{Ph}), 139.3(\mathrm{Ph}), 140.4(\mathrm{Ph}), 141.1$ $(\mathrm{Ph}), 141.8(\mathrm{Ph}), 145.0(\mathrm{Ph}), 147.8(\mathrm{Ph}), 148.4$ (Ph), $151.2(\mathrm{Ph}), 151.4(\mathrm{Ph}), 161.5(\mathrm{Ph}), 163.5$ $(\mathrm{Ph}), 167.5(\mathrm{Ph})$.

${ }^{19}$ F NMR $\left(470 \mathrm{MHz}, \mathrm{CD}_{2} \mathrm{Cl}_{2}, 238 \mathrm{~K}\right): \delta / \mathrm{ppm}=-115.3(\mathrm{~m})$.

\section{${ }^{\mathrm{MeO}} \mathrm{LH}_{4}$}

Empirical Formula:

Molecular Weight $(\mathrm{g} / \mathrm{mol})$ :

Yield:
$\mathrm{C}_{98} \mathrm{H}_{96} \mathrm{~N}_{8} \mathrm{O}_{6}$

1481.90

$66.7 \mathrm{mg}, 14.5 \%$ 
$\mathbf{R}_{f}$ (silica, $\left.\mathrm{MeOH}\right): \quad 0.56$

HRMS $\left(\mathrm{ESI}^{+}, \mathrm{MeOH}\right): m / z=\quad 1481.7526($ calcd. 1481.7526$)[\mathrm{M}+\mathrm{H}]^{+}, 741.3794$

IR $(\mathrm{KBr})$ :

(calcd. 741.3799) $[\mathrm{M}+2 \mathrm{H}]^{2+}$.

$\widetilde{v}\left(\mathrm{~cm}^{-1}\right)=3074(\mathrm{w}), 3055(\mathrm{w}), 3022(\mathrm{w})$,

2955 (s), 2925 (vs), 2868 (m), 2853 (s),

$1693(\mathrm{~m}), 1646$ (m), $1583(\mathrm{~m}), 1554(\mathrm{w})$,

$1531(\mathrm{w}), 1519(\mathrm{~m}), 1501(\mathrm{~s}), 1464(\mathrm{~s}), 1409(\mathrm{~m})$,

$1389(\mathrm{~m}), 1330(\mathrm{~s}), 1235(\mathrm{~s}), 1181(\mathrm{~m}), 1125(\mathrm{w})$,

$1053(\mathrm{~m}), 1009(\mathrm{~m}), 920(\mathrm{w}), 829(\mathrm{w}), 766(\mathrm{w})$,

$697(\mathrm{~s}), 509(\mathrm{w}), 375(\mathrm{w})$.

UV-vis $/ \mathbf{n m}\left(\epsilon / \mathrm{M}^{-1} \mathrm{~cm}^{-1}\right): \quad 278$ (37200), 306 (38700), 390 (86800), 640 (33500), 734 (15000).

${ }^{\mathbf{1}} \mathbf{H} \mathbf{N M R}\left(500 \mathrm{MHz}, \mathrm{CD}_{2} \mathrm{Cl}_{2}, 238 \mathrm{~K}\right): \delta / \mathrm{ppm}=0.44\left(\mathrm{t},{ }^{3} J(\mathrm{H}-\mathrm{H})=7.3 \mathrm{~Hz}, 3 \mathrm{H}\right.$, $\left.\mathrm{CH}_{3}\right), 0.52\left(\mathrm{t},{ }^{3} J(\mathrm{H}-\mathrm{H})=7.3 \mathrm{~Hz}, 3 \mathrm{H}, \mathrm{CH}_{3}\right)$, $0.56\left(\mathrm{t},{ }^{3} \mathrm{~J}(\mathrm{H}-\mathrm{H})=7.3 \mathrm{~Hz}, 3 \mathrm{H}, \mathrm{CH}_{3}\right), 0.65$ $\left(\mathrm{t},{ }^{3} J(\mathrm{H}-\mathrm{H})=7.3 \mathrm{~Hz}, 3 \mathrm{H}, \mathrm{CH}_{3}\right), 1.10(\mathrm{q}$, $\left.{ }^{3} J(\mathrm{H}-\mathrm{H})=7.3 \mathrm{~Hz}, 1 \mathrm{H}, \mathrm{CH}_{2}\right), 1.30\left(\mathrm{q},{ }^{3} J(\mathrm{H}-\right.$ $\left.\mathrm{H})=7.3 \mathrm{~Hz}, 1 \mathrm{H}, \mathrm{CH}_{2}\right), 1.38-1.45\left(\mathrm{q}, 2 \mathrm{H}, \mathrm{CH}_{2}\right)$, $1.56\left(\mathrm{q},{ }^{3} J(\mathrm{H}-\mathrm{H})=7.3 \mathrm{~Hz}, 1 \mathrm{H}, \mathrm{CH}_{2}\right), 1.63$ $\left(\mathrm{q},{ }^{3} J(\mathrm{H}-\mathrm{H})=7.3 \mathrm{~Hz}, 1 \mathrm{H}, \mathrm{CH}_{2}\right), 1.90(\mathrm{q}$, $\left.{ }^{3} J(\mathrm{H}-\mathrm{H})=7.3 \mathrm{~Hz}, 1 \mathrm{H}, \mathrm{CH}_{2}\right), 2.01\left(\mathrm{q},{ }^{3} J(\mathrm{H}-\right.$ $\left.\mathrm{H})=7.3 \mathrm{~Hz}, 1 \mathrm{H}, \mathrm{CH}_{2}\right), 3.68\left(\mathrm{~s}, 3 \mathrm{H}, \mathrm{OCH}_{3}\right), 3.71$ $\left(\mathrm{s}, 3 \mathrm{H}, \mathrm{OCH}_{3}\right), 3.79\left(\mathrm{~s}, 3 \mathrm{H}, \mathrm{OCH}_{3}\right), 6.13(\mathrm{dd}$, $\left.{ }^{4} J(\mathrm{H}-\mathrm{H})=2.0 \mathrm{~Hz},{ }^{3} J(\mathrm{H}-\mathrm{H})=7.5 \mathrm{~Hz}, 1 \mathrm{H}, \mathrm{Ph}\right)$, $6.21\left(\mathrm{~d},{ }^{4} J(\mathrm{H}-\mathrm{H})=1.5 \mathrm{~Hz}, 1 \mathrm{H}, \mathrm{Ph}\right), 6.31(\mathrm{td}$, $\left.{ }^{4} J(\mathrm{H}-\mathrm{H})=1.3 \mathrm{~Hz},{ }^{3} J(\mathrm{H}-\mathrm{H})=7.5 \mathrm{~Hz}, 1 \mathrm{H}, \mathrm{Ph}\right)$, $6.43\left(\mathrm{~d},{ }^{4} J(\mathrm{H}-\mathrm{H})=1.5 \mathrm{~Hz}, 1 \mathrm{H}, \mathrm{Ph}\right), 6.54(\mathrm{td}$, $\left.{ }^{5} J(\mathrm{H}-\mathrm{H})=1.3 \mathrm{~Hz},{ }^{3} J(\mathrm{H}-\mathrm{H})=7.5 \mathrm{~Hz}, 1 \mathrm{H}, \mathrm{Ph}\right)$, $6.72\left(\mathrm{td},{ }^{4} J(\mathrm{H}-\mathrm{H})=1.5 \mathrm{~Hz},{ }^{3} J(\mathrm{H}-\mathrm{H})=7.5 \mathrm{~Hz}\right.$, $1 \mathrm{H}, \mathrm{Ph}), 6.78\left(\mathrm{td},{ }^{4} J(\mathrm{H}-\mathrm{H})=1.5 \mathrm{~Hz},{ }^{3} J(\mathrm{H}-\right.$ $\mathrm{H})=7.5 \mathrm{~Hz}, 1 \mathrm{H}, \mathrm{Ph}), 6.83\left(\mathrm{dd},{ }^{4} J(\mathrm{H}-\right.$ $\left.\mathrm{H})=1.5 \mathrm{~Hz},{ }^{3} J(\mathrm{H}-\mathrm{H})=7.5 \mathrm{~Hz}, 1 \mathrm{H}, \mathrm{Ph}\right), 6.96$ $\left(\mathrm{td},{ }^{4} J(\mathrm{H}-\mathrm{H})=1.5 \mathrm{~Hz},{ }^{4} J(\mathrm{H}-\mathrm{H})=7.5 \mathrm{~Hz}\right.$, $1 \mathrm{H}, \mathrm{Ph}), 7.00\left(\mathrm{dd},{ }^{4} J(\mathrm{H}-\mathrm{H})=1.5 \mathrm{~Hz},{ }^{3} J(\mathrm{H}-\right.$ $\mathrm{H})=7.5 \mathrm{~Hz}, 1 \mathrm{H}, \mathrm{Ph}), 7.21-7.35(\mathrm{~m}, 5 \mathrm{H}, \mathrm{Ph})$, 7.48-7.54 (m, 2H, Ph), 11.49 (s, 1H, NH $\left.{ }^{\mathrm{pyr}}\right)$, $13.20\left(\mathrm{~s}, 1 \mathrm{H}, \mathrm{NH}^{\mathrm{pz}}\right)$. 
${ }^{13} \mathrm{C} \mathrm{NMR}\left(125 \mathrm{MHz}, \mathrm{CD}_{2} \mathrm{Cl}_{2}, 238 \mathrm{~K}\right): \delta / \mathrm{ppm}=14.7\left(\mathrm{CH}_{3}\right), 15.9\left(\mathrm{CH}_{3}\right), 16.0\left(\mathrm{CH}_{3}\right)$, $16.7\left(\mathrm{CH}_{3}\right), \quad 18.1\left(2 \mathrm{C}, \mathrm{CH}_{2}\right), \quad 18.5\left(\mathrm{CH}_{2}\right)$, $18.9\left(\mathrm{CH}_{2}\right), 55.7\left(\mathrm{OCH}_{3}\right), 55.9\left(\mathrm{OCH}_{3}\right), 60.9$ $\left(\mathrm{OCH}_{3}\right), 105.9(\mathrm{Ph}), 108.8(\mathrm{Ph}), 109.6(\mathrm{Ph})$, $114.8(\mathrm{Ph}), 123.9(\mathrm{Ph}), 126.1(\mathrm{Ph}), 126.4(\mathrm{Ph})$, $126.6(\mathrm{Ph}), 127.0(\mathrm{Ph}), 127.2(\mathrm{Ph}), 127.3(\mathrm{Ph})$, $127.7(\mathrm{Ph}), 127.7(\mathrm{Ph}), 127.8(\mathrm{Ph}), 127.9(\mathrm{Ph})$, $130.8(\mathrm{Ph}), 131.7(\mathrm{Ph}), 132.5(\mathrm{Ph}), 132.6(\mathrm{Ph})$, $133.2(\mathrm{Ph}), 133.7(\mathrm{Ph}), 134.1(\mathrm{Ph}), 135.3(\mathrm{Ph})$, $137.1(\mathrm{Ph}), 139.1(\mathrm{Ph}), 139.5(\mathrm{Ph}), 140.7(\mathrm{Ph})$, $141.2(\mathrm{Ph}), 141.7(\mathrm{Ph}), 145.3(\mathrm{Ph}), 147.8(\mathrm{Ph})$, $148.4(\mathrm{Ph}), 151.1(\mathrm{Ph}), 151.4(\mathrm{Ph}), 151.7(\mathrm{Ph})$, $152.3(\mathrm{Ph}), 167.1(\mathrm{Ph})$.

\section{${ }^{p \mathrm{MeO} \mathrm{LH}_{4}}$}

Empirical Formula:

$\mathrm{C}_{94} \mathrm{H}_{88} \mathrm{~N}_{8} \mathrm{O}_{2}$

Molecular Weight $(\mathrm{g} / \mathrm{mol})$ :

1361.79

Yield:

$113.1 \mathrm{mg}, 26.8 \%$

$\mathbf{R}_{f}$ (silica, $\left.\mathrm{MeOH}\right)$ :

0.32

HRMS $\left(\mathrm{ESI}^{+}, \mathrm{MeOH}\right): m / z=$ 1361.7104 (calcd. 1361.7103) $[\mathrm{M}+\mathrm{H}]^{+}, 681.3582$ (calcd. 681.3588) $[\mathrm{M}+2 \mathrm{H}]^{2+}$.

IR $(\mathrm{KBr})$ :

$\widetilde{v}\left(\mathrm{~cm}^{-1}\right)=3074(\mathrm{w}), 3055(\mathrm{w}), 3022(\mathrm{w})$, 2960 (s), 2925 (vs), 2853 (s), 1744 (m), 1636 (vs), 1611 (vs), 1520 (m), 1462 (m), 1385 (s), 1366 (m), 1283 (w), 1254 (m), $1175(\mathrm{~m}), 1112(\mathrm{~s}), 1029(\mathrm{~s}), 857(\mathrm{w}), 803(\mathrm{~m})$, $768(\mathrm{w}), 697(\mathrm{~m}), 670(\mathrm{w}), 620(\mathrm{w}), 584(\mathrm{w})$, $544(\mathrm{w}), 475(\mathrm{w})$.

UV-vis $/ \mathbf{n m}\left(\epsilon / \mathrm{M}^{-1} \mathrm{~cm}^{-1}\right)$ : 277 (33500), 306 (33600), 390 (91000), 640 (39600), 734 (14700). 
${ }^{\mathbf{1}} \mathbf{H}$ NMR $\left(500 \mathrm{MHz}, \mathrm{CD}_{2} \mathrm{Cl}_{2}, 238 \mathrm{~K}\right): \delta / \mathrm{ppm}=0.43\left(\mathrm{t},{ }^{3} J(\mathrm{H}-\mathrm{H})=7.3 \mathrm{~Hz}, 3 \mathrm{H}, \mathrm{CH}_{3}\right)$, $0.45\left(\mathrm{t},{ }^{3} \mathrm{~J}(\mathrm{H}-\mathrm{H})=7.3 \mathrm{~Hz}, 3 \mathrm{H}, \mathrm{CH}_{3}\right), 0.56$ $\left(\mathrm{t},{ }^{3} \mathrm{~J}(\mathrm{H}-\mathrm{H})=7.3 \mathrm{~Hz}, 6 \mathrm{H}, \mathrm{CH}_{3}\right), 0.94(\mathrm{q}$, $\left.{ }^{3} J(\mathrm{H}-\mathrm{H})=7.3 \mathrm{~Hz}, 1 \mathrm{H}, \mathrm{CH}_{2}\right), 1.24\left(\mathrm{q},{ }^{3} J(\mathrm{H}-\right.$ $\left.\mathrm{H})=7.3 \mathrm{~Hz}, 1 \mathrm{H}, \mathrm{CH}_{2}\right), 1.44\left(\mathrm{q}, 2 \mathrm{H}, \mathrm{CH}_{2}\right)$, $1.56\left(\mathrm{q},{ }^{3} J(\mathrm{H}-\mathrm{H})=7.3 \mathrm{~Hz}, 2 \mathrm{H}, \mathrm{CH}_{2}\right), 1.87$ (q, $\left.{ }^{3} J(\mathrm{H}-\mathrm{H})=7.3 \mathrm{~Hz}, 2 \mathrm{H}, \mathrm{CH}_{2}\right), 1.98\left(\mathrm{q},{ }^{3} J(\mathrm{H}-\right.$ $\left.\mathrm{H})=7.3 \mathrm{~Hz}, 1 \mathrm{H}, \mathrm{CH}_{2}\right), 3.76\left(\mathrm{~s}, 3 \mathrm{H}, \mathrm{OCH}_{3}\right)$, $6.17\left(\mathrm{dd},{ }^{4} J(\mathrm{H}-\mathrm{H})=2.0 \mathrm{~Hz},{ }^{3} J(\mathrm{H}-\mathrm{H})=7.5 \mathrm{~Hz}\right.$, $1 \mathrm{H}, \mathrm{Ph}), 6.29\left(\mathrm{td},{ }^{4} J(\mathrm{H}-\mathrm{H})=1.3 \mathrm{~Hz},{ }^{3} J(\mathrm{H}-\right.$ $\mathrm{H})=7.5 \mathrm{~Hz}, 1 \mathrm{H}, \mathrm{Ph}), 6.55\left(\mathrm{td},{ }^{4} J(\mathrm{H}-\right.$ $\left.\mathrm{H})=1.3 \mathrm{~Hz},{ }^{3} J(\mathrm{H}-\mathrm{H})=7.5 \mathrm{~Hz}, 1 \mathrm{H}, \mathrm{Ph}\right), 6.69-$ $6.76(\mathrm{~m}, 3 \mathrm{H}, \mathrm{Ph}), 6.79\left(\mathrm{td},{ }^{4} J(\mathrm{H}-\mathrm{H})=1.5 \mathrm{~Hz}\right.$, $\left.{ }^{3} J(\mathrm{H}-\mathrm{H})=7.5 \mathrm{~Hz}, 1 \mathrm{H}, \mathrm{Ph}\right), 6.84\left(\mathrm{dd},{ }^{4} J(\mathrm{H}-\right.$ $\left.\mathrm{H})=1.5 \mathrm{~Hz},{ }^{3} J(\mathrm{H}-\mathrm{H})=7.5 \mathrm{~Hz}, 1 \mathrm{H}, \mathrm{Ph}\right), 6.93$ $\left(\mathrm{dd},{ }^{4} J(\mathrm{H}-\mathrm{H})=2.0 \mathrm{~Hz},{ }^{3} J(\mathrm{H}-\mathrm{H})=7.5 \mathrm{~Hz}\right.$, $1 \mathrm{H}, \mathrm{Ph}), 6.97\left(\mathrm{td},{ }^{4} J(\mathrm{H}-\mathrm{H})=1.5 \mathrm{~Hz},{ }^{4} J(\mathrm{H}-\right.$ $\mathrm{H})=7.5 \mathrm{~Hz}, 1 \mathrm{H}, \mathrm{Ph}), 7.02\left(\mathrm{dd},{ }^{4} J(\mathrm{H}-\right.$ $\left.\mathrm{H})=1.5 \mathrm{~Hz},{ }^{3} J(\mathrm{H}-\mathrm{H})=7.5 \mathrm{~Hz}, 1 \mathrm{H}, \mathrm{Ph}\right), 7.06$ $\left(\mathrm{dd},{ }^{4} J(\mathrm{H}-\mathrm{H})=1.5 \mathrm{~Hz},{ }^{3} J(\mathrm{H}-\mathrm{H})=7.5 \mathrm{~Hz}, 1 \mathrm{H}\right.$, $\mathrm{Ph})$ 7.21-7.37 (m, 5H, Ph), 7.49-7.55 (m, 2H, $\mathrm{Ph}), 11.33$ (s, 1H, NH $\left.{ }^{\mathrm{pyr}}\right), 13.37$ (s, 1H, $\left.\mathrm{NH}^{\mathrm{pz}}\right)$.

${ }^{13} \mathrm{C}$ NMR $\left(125 \mathrm{MHz}, \mathrm{CD}_{2} \mathrm{Cl}_{2}, 238 \mathrm{~K}\right): \delta / \mathrm{ppm}=14.8\left(\mathrm{CH}_{3}\right), 15.6\left(\mathrm{CH}_{3}\right), 16.0\left(\mathrm{CH}_{3}\right)$, $16.3\left(\mathrm{CH}_{3}\right), 18.1\left(2 \mathrm{C}, \mathrm{CH}_{2}\right), 18.2\left(\mathrm{CH}_{2}\right), 18.5$ $\left(\mathrm{CH}_{2}\right), 18.9\left(\mathrm{CH}_{2}\right), 55.3\left(\mathrm{OCH}_{3}\right), 105.5(\mathrm{Ph})$, $112.4(\mathrm{Ph}), 112.5(\mathrm{Ph}), 113.9(\mathrm{Ph}), 123.9(\mathrm{Ph})$, $124.2(\mathrm{Ph}), 124.4(\mathrm{Ph}), 126.1(\mathrm{Ph}), 126.5(\mathrm{Ph})$, $126.5(\mathrm{Ph}), 127.0(\mathrm{Ph}), 127.2(\mathrm{Ph}), 127.2(\mathrm{Ph})$, $127.7(\mathrm{Ph}), 127.7(\mathrm{Ph}), 127.9(\mathrm{Ph}), 130.1(\mathrm{Ph})$, $130.7(\mathrm{Ph}), 131.6(\mathrm{Ph}), 132.4(\mathrm{Ph}), 132.4(\mathrm{Ph})$, $133.1(\mathrm{Ph}), 133.5(\mathrm{Ph}), 133.9(\mathrm{Ph}), 134.1(\mathrm{Ph})$, $135.3(\mathrm{Ph}), 139.1(\mathrm{Ph}), 139.4(\mathrm{Ph}), 140.7(\mathrm{Ph})$, $141.3(\mathrm{Ph}), 141.4(\mathrm{Ph}), 145.3(\mathrm{Ph}), 147.5(\mathrm{Ph})$, $148.4(\mathrm{Ph}), 151.2(\mathrm{Ph}), 151.4(\mathrm{Ph}), 159.0(\mathrm{Ph})$, $167.8(\mathrm{Ph})$. 


\subsection{Complex Synthesis}

\subsubsection{General Procedure for the Synthesis of Copper Complexes ${ }^{\mathrm{X}} \mathrm{LCu}_{2}$}
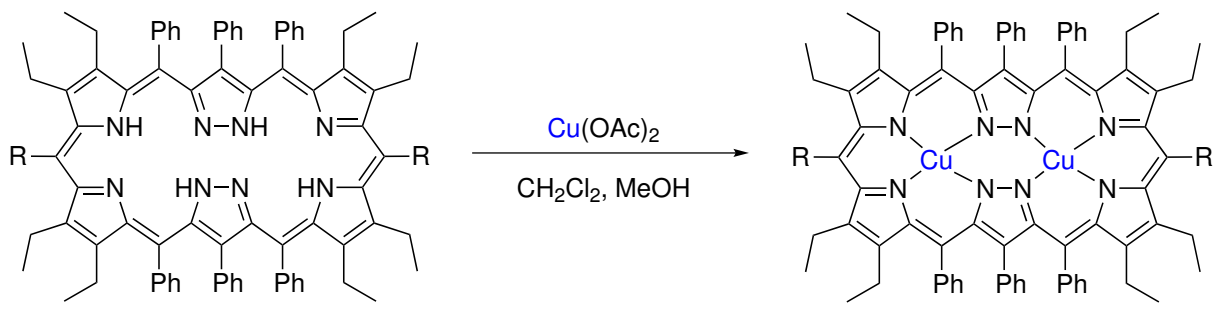

Copper acetate $(62.7 \mathrm{mg}, 345.6 \mu \mathrm{mol}, 9.0 \mathrm{eq}$.) was added to a solution of the SiameseTwin Porphyrin $\mathbf{X}_{\mathbf{L H}} \mathbf{4}(38.4 \mu \mathrm{mol}, 1.0$ eq.) in dichloromethane $(10.0 \mathrm{~mL})$ and methanol $(20 \mathrm{~mL})$ and stirred for $30 \mathrm{~min}$ at ambient temperature. The solvent was removed under reduced pressure and the residue was dissolved in dichloromethane and filtered over a plug of basic aluminum oxide. The only fraction passing was the product. The copper complex of the Siamese-twin porphyrin ${ }^{\mathbf{X}} \mathbf{L} \mathbf{C u}_{2}$ was obtained as a blue solid.

${ }^{p \mathrm{Me}} \mathrm{LCu}_{2}$

\section{Empirical Formula:}

Molecular Weight $(\mathrm{g} / \mathrm{mol})$ :

Yield:

$\mathbf{R}_{f}$ (silica, $n$-hexane/EtOAc 2:1):

HRMS $\left(\mathrm{ESI}^{+}, \mathrm{MeOH}\right): m / z=$

IR $(\mathrm{KBr})$ :

UV-vis/nm $\left(\epsilon / \mathrm{M}^{-1} \mathrm{~cm}^{-1}\right)$ :
$\mathrm{C}_{94} \mathrm{H}_{84} \mathrm{~N}_{8} \mathrm{Cu}_{2}$

1452.85

$33.6 \mathrm{mg}, 60.3 \%$

0.82

1450.5394 (calcd. 1450.5405) $[\mathrm{M}]^{+}, 1473.5296$

(calcd. 1473.5303) $[\mathrm{M}+\mathrm{Na}]^{+}$.

$\widetilde{v}\left(\mathrm{~cm}^{-1}\right)=3078(\mathrm{w}), 3052(\mathrm{w}), 3022(\mathrm{w}), 2957$

(s), $2927(\mathrm{~s}), 2870(\mathrm{~s}), 1741$ (w), 1651 (m), 1639

(m), 1602 (m), 1580 (w), 1560 (w), 1512 (m),

1439 (vs), 1372 (m), 1355 (w), 1302 (s), 1252

(w), $1228(\mathrm{w}), 1190(\mathrm{~s}), 1154(\mathrm{~m}), 1112(\mathrm{~s}), 1056$

(w), $1018(\mathrm{~m}), 960(\mathrm{w}), 928(\mathrm{w}), 890(\mathrm{w}), 860$

(w), $814(\mathrm{~m}), 769(\mathrm{~m}), 722(\mathrm{~m}), 695(\mathrm{~s}), 617$

$(\mathrm{w}), 562(\mathrm{w}), 546(\mathrm{w}), 512(\mathrm{w}), 462(\mathrm{w}), 390$ (w).

284 (31700), 391 (55900), 578 (13400), 636 (24000), 682 (18200).

\section{${ }^{p \mathrm{~F}} \mathrm{LCu}_{2}$}

Empirical Formula:

Molecular Weight $(\mathrm{g} / \mathrm{mol})$ :

Yield:
$\mathrm{C}_{92} \mathrm{H}_{78} \mathrm{~N}_{8} \mathrm{~F}_{2} \mathrm{Cu}_{2}$

1460.78

$10.3 \mathrm{mg}, 18.3 \%$ 
$\mathbf{R}_{f}$ (silica, $n$-hexane/EtOAc 2:1): 0.83

HRMS $\left(\mathrm{ESI}^{+}, \mathrm{MeOH}\right): m / z=\quad 1458.4890\left(\right.$ calcd. 1458.4904) $[\mathrm{M}]^{+}$.

IR $(\mathrm{KBr})$ :

$\widetilde{v}\left(\mathrm{~cm}^{-1}\right)=3056(\mathrm{w}), 3005(\mathrm{w}), 2961(\mathrm{~s}), 2924$

(vs), $2853(\mathrm{~s}), 1737(\mathrm{w}), 1656(\mathrm{~s}), 1634(\mathrm{~s}), 1601$

$(\mathrm{m}), 1535(\mathrm{w}), 1509(\mathrm{~m}), 1468(\mathrm{~m}), 1442(\mathrm{~m})$

1405 (s), 1379 (w), 1348 (w), $1300(\mathrm{w}), 1261$

(s), $1222(\mathrm{w}), 1153(\mathrm{w}), 1095(\mathrm{vs}), 1022(\mathrm{vs})$, $865(\mathrm{w}), 804(\mathrm{~s}), 720(\mathrm{w}), 696(\mathrm{~m}), 668(\mathrm{w}), 465$ $(\mathrm{m}), 391(\mathrm{~m}), 365(\mathrm{~m})$.

UV-vis $/ \mathbf{n m}\left(\epsilon / \mathrm{M}^{-1} \mathrm{~cm}^{-1}\right)$ :

300 (30700), 390 (59100), 549 (14400), 632 (25400), 682 (19200).

\section{${ }^{\mathrm{MeO}} \mathrm{LCu}_{2}$}

Empirical Formula:

Molecular Weight $(\mathrm{g} / \mathrm{mol})$ :

Yield:

$\mathbf{R}_{f}$ (silica, $n$-hexane/EtOAc 2:1):

HRMS $\left(\mathrm{ESI}^{+}, \mathrm{MeOH}\right): m / z=$

IR $(\mathrm{KBr})$ :

$\mathbf{U V - v i s / n m}\left(\epsilon / \mathrm{M}^{-1} \mathrm{~cm}^{-1}\right)$ :
$\mathrm{C}_{98} \mathrm{H}_{92} \mathrm{~N}_{8} \mathrm{O}_{6} \mathrm{Cu}_{2}$

1604.96

$41.1 \mathrm{mg}, 66.7 \%$

0.58

1602.5720 (calcd. 1602.5726) $[\mathrm{M}]^{+}, 1625.5618$ (calcd. 1625.5624) $[\mathrm{M}+\mathrm{Na}]^{+}$.

$\widetilde{v}\left(\mathrm{~cm}^{-1}\right)=3055(\mathrm{w}), 3022(\mathrm{w}), 2961(\mathrm{~m}), 2927$

(s), $2870(\mathrm{~m}), 1737(\mathrm{w}), 1656(\mathrm{~m}), 1639(\mathrm{~m})$, 1599 (w), 1577 (m), 1501 (s), 1436 (vs), 1405 (s), $1373(\mathrm{~m}), 1341(\mathrm{~s}), 1297(\mathrm{w}), 1235(\mathrm{~m}), 1180$ (m), 1127 (vs), 1111 (vs), 1054 (m), 1009 (s), $954(\mathrm{w}), 927(\mathrm{w}), 898(\mathrm{w}), 830(\mathrm{w}), 811(\mathrm{~m})$, $770(\mathrm{~m}), 750(\mathrm{~m}), 695(\mathrm{~s}), 632(\mathrm{w}), 572(\mathrm{w}), 541$ $(\mathrm{w}), 460(\mathrm{w}), 390(\mathrm{w})$.

300 (31300), 389 (57100), 546 (20000), 632 (21000), 682 (18000).

$\mathrm{C}_{94} \mathrm{H}_{84} \mathrm{~N}_{8} \mathrm{O}_{2} \mathrm{Cu}_{2}$

1484.85

$29.4 \mathrm{mg}, 51.6 \%$

0.73

1482.5295 (calcd. 1482.5304) $[\mathrm{M}]^{+}$. 
IR $(\mathrm{KBr})$ :

UV-vis $/ \mathbf{n m}\left(\epsilon / \mathrm{M}^{-1} \mathrm{~cm}^{-1}\right)$ : $\widetilde{v}\left(\mathrm{~cm}^{-1}\right)=3056(\mathrm{w}), 3024(\mathrm{w}), 2966(\mathrm{~s}), 2929$

(s), $2871(\mathrm{~m}), 1655(\mathrm{~s}), 1605(\mathrm{~m}), 1567(\mathrm{w}), 1542$

(w), 1512 (vs), 1489 (m), 1437 (vs), 1402 (m),

$1376(\mathrm{~m}), 1348(\mathrm{w}), 1258(\mathrm{~s}), 1243(\mathrm{vs}), 1175$ (s),

$1148(\mathrm{~m}), 1110(\mathrm{~s}), 1057(\mathrm{w}), 1019(\mathrm{~m}), 1002$

$(\mathrm{m}), 958(\mathrm{w}), 934(\mathrm{~m}), 910(\mathrm{w}), 893(\mathrm{w}), 827$

(m), $750(\mathrm{w}), 727(\mathrm{w}), 695(\mathrm{vs}), 606(\mathrm{w}), 548$

$(\mathrm{w}), 372(\mathrm{w})$.

291 (34200), 389 (58500), 542 (21500), 639

(21900), 682 (19200).

\subsubsection{Iron(III) Nickel(II) Complex LNiFeCI}

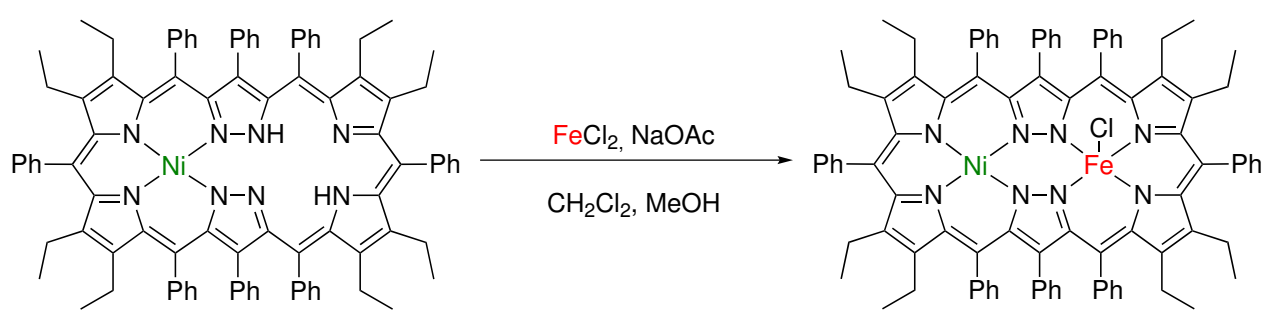

$\mathrm{FeCl}_{2}(84.0 \mathrm{mg}, 660 \mu \mathrm{mol}, 6.0$ eq.) and $\mathrm{NaOAc}(81.0 \mathrm{mg}, 990 \mu \mathrm{mol}, 9.0$ eq.) were added to a solution of $\mathbf{L H}_{2} \mathbf{N i}(150 \mathrm{mg}, 110 \mu \mathrm{mol}, 1.0$ eq.) in dichloromethane $(15 \mathrm{~mL})$ and $\mathrm{MeOH}(15 \mathrm{~mL})$. The mixture was stirred for 15 minutes at ambient temperature. After removing of the solvent under reduced pressure, $\mathrm{CH}_{2} \mathrm{Cl}_{2}(20 \mathrm{~mL})$ was added and the suspension was filtered. The filtrate was filtered over basic aluminum oxide, where the product was the only fraction passing, and the solvent was removed under reduced pressure. The raw product was recrystallized from a mixture of $\mathrm{CH}_{2} \mathrm{Cl}_{2}$ and $n$-hexane (1:1) to yield $110 \mathrm{mg}$ (69\%) LNiFeCl. Crystals suitable for X-Ray crystallography were obtained by slow evaporation of a saturated solution of $\mathbf{L N i F e C l}$ in acetone into dimethylformamide.

Empirical Formula:

Molecular Weight $(\mathrm{g} / \mathrm{mol})$ :

HRMS $\left(\mathrm{ESI}^{+}, \mathrm{MeOH}\right): m / z=$

IR $(\mathrm{KBr})$ :

\author{
$\mathrm{C}_{92} \mathrm{H}_{80} \mathrm{~N}_{8} \mathrm{NiFeCl}$ \\ 1447.67 \\ 1410.5207 (calcd. 1410.5207) $[\mathrm{M}]^{+}$. \\ $\widetilde{v}\left(\mathrm{~cm}^{-1}\right)=3054(\mathrm{w}), 3022(\mathrm{w}), 2973(\mathrm{~m}) 2957$ \\ (m), 2929 (s), 2870 (m), 1639 (w), 1597 (m), \\ 1565 (m), 1545 (m), 1525 (m), 1494 (m), 1454 \\ (vs), 1441 (vs), 1423 (vs), 1375 (m), 1351 (m), \\ $1317(\mathrm{w}), 1252(\mathrm{~m}), 1180(\mathrm{~m}), 1157(\mathrm{w}), 1111$ \\ (m), $1072(\mathrm{w}), 1053(\mathrm{~m}), 1015(\mathrm{~m}), 932(\mathrm{w})$, \\ $895(\mathrm{w}), 843(\mathrm{w}), 810(\mathrm{w}), 772(\mathrm{w}), 746(\mathrm{~m})$, \\ $661(\mathrm{w}), 616(\mathrm{w}), 554(\mathrm{w})$.
}


UV-vis/nm $\left(\epsilon / \mathrm{M}^{-1} \mathrm{~cm}^{-1}\right)$ :

Elemental Analysis (\%):
380 (67400), 535 (22700), 700 (16600).

Found (calcd.) for $\mathrm{C}_{92} \mathrm{H}_{80} \mathrm{~N}_{8} \mathrm{NiFeCl} \cdot 2 n$ hexane $\cdot 0.75 \mathrm{CH}_{2} \mathrm{Cl}_{2}$ : $\mathrm{C} 74.74(74.72), \mathrm{H} 6.56$

(6.55), N 6.65 (6.65).

\subsubsection{Iron(II) Nickel(II) Complex LNiFe}
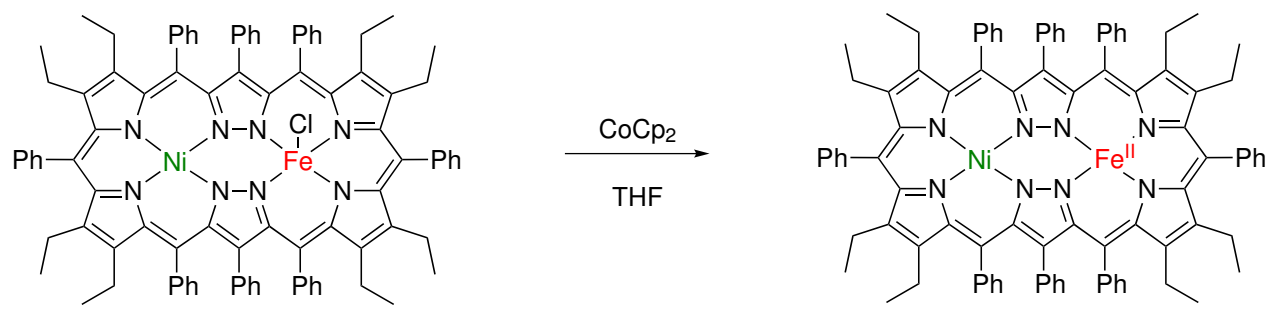

A solution of $\mathrm{CoCp}_{2}$ in dry THF $(100 \mu \mathrm{L}, 14.7 \mathrm{~mm}, 1.0$ eq.) was added to a solution of LNiFeCl (2 mg, $1.47 \mu \mathrm{mol}, 1.0$ eq.) in dry THF under inert conditions at $-40{ }^{\circ} \mathrm{C}$. The solution was stirred for 5 minutes and directly used for further reactions and analysis.

$$
\begin{array}{ll}
\text { Empirical Formula: } & \mathrm{C}_{92} \mathrm{H}_{80} \mathrm{~N}_{8} \mathrm{NiFe} \\
\text { Molecular Weight }(\mathrm{g} / \mathrm{mol}): & 1412.21 \\
\text { UV-vis } / \mathbf{n m}\left(\epsilon / \mathrm{M}^{-1} \mathrm{~cm}^{-1}\right): & 380(60300), 665(18100) .
\end{array}
$$

\subsection{4 ${ }^{57}$ Iron(III) Nickel(II) Complex $\mathrm{LNi}^{57} \mathrm{FeCl}$}

The isotopically labeled $\mathbf{L N i}{ }^{57} \mathbf{F e C l}$ was synthesized like the corresponding non labeled nickel iron complex $\mathbf{L N i F e C l}$ by the use of ${ }^{57} \mathrm{FeCl}_{2} .{ }^{57} \mathrm{FeCl}_{2}$ was synthesized from ${ }^{57} \mathrm{Fe}$ by the addition of concentrated $\mathrm{HCl}$ and stirring until no metal gloss was observable anymore. The remaining liquid was removed and the obtained ${ }^{57} \mathrm{FeCl}_{2}$ was directly used for the complexation reaction.

\subsection{5 ${ }^{57}$ Iron(II) Nickel(II) Complex $\mathrm{LNi}^{57} \mathrm{Fe}$}

$\mathbf{L N i}^{57} \mathbf{F e}$ was synthesized like the corresponding non labeled reduced nickel iron complex $\mathbf{L N i F e}$ by using the labeled $\mathbf{L N i}{ }^{\mathbf{5 7}} \mathbf{F e C l}$. The obtained solution in THF was directly used for further reactions and characterization. 


\subsubsection{Monoiron Complex of the Siamese-Twin Porphyrin $\mathrm{LH}_{2} \mathrm{Fe}$}

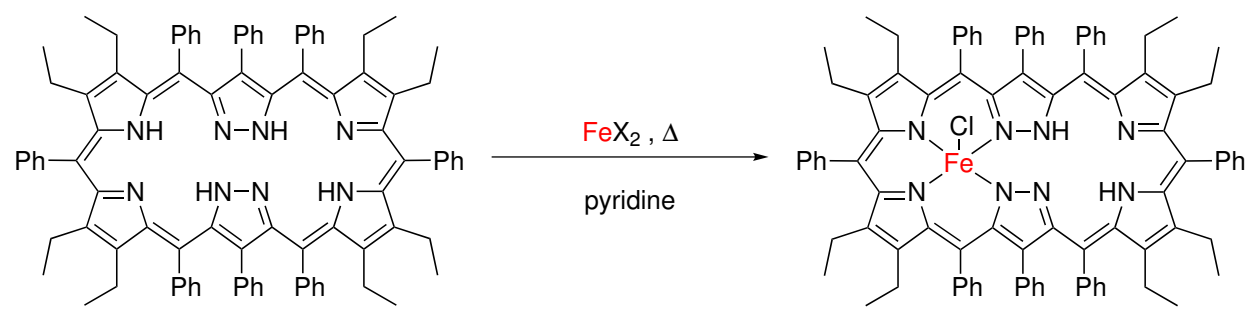

The free base porphyrin $\mathbf{L H}_{\mathbf{4}}(50.0 \mathrm{mg}, 38.4 \mu \mathrm{mol}, 1.0$ eq. $)$ was dissolved in pyridine $(25 \mathrm{~mL})$ and $\mathrm{FeCl}_{2}(73.0 \mathrm{mg}, 576.0 \mu \mathrm{mol}, 15.0$ eq. $)$ was added. The reaction mixture was stirred at $60{ }^{\circ} \mathrm{C}$ for 15 minutes and the solvent was removed under reduced pressure. Toluene $(10 \mathrm{~mL})$ was added and removed two times. The residue was suspended in toluene and filtered and the solvent was removed under reduced pressure. The obtained crude mixture of $\mathbf{L H}_{4}, \mathbf{L}\{\mathbf{F e C l}\}_{2}$ and $\mathbf{L H}_{2} \mathbf{F e C l}$ was directly used for analysis and further reactions.

Empirical Formula:

Molecular Weight $(\mathrm{g} / \mathrm{mol})$ :

HRMS $\left(\mathrm{ESI}^{+}, \mathrm{MeOH}\right): m / z=$
$\mathrm{C}_{92} \mathrm{H}_{82} \mathrm{~N}_{8} \mathrm{FeCl}$

1390.99

1390.5778 (calcd. 1390.5777$) \quad[\mathrm{M}+\mathrm{Cl}+\mathrm{H}]^{+}$, 1354.6002 (calcd. 1354.6010) $[\mathrm{M}]^{+}$.

\subsubsection{Iron(III) Copper(II) Complex LCuFeCl}

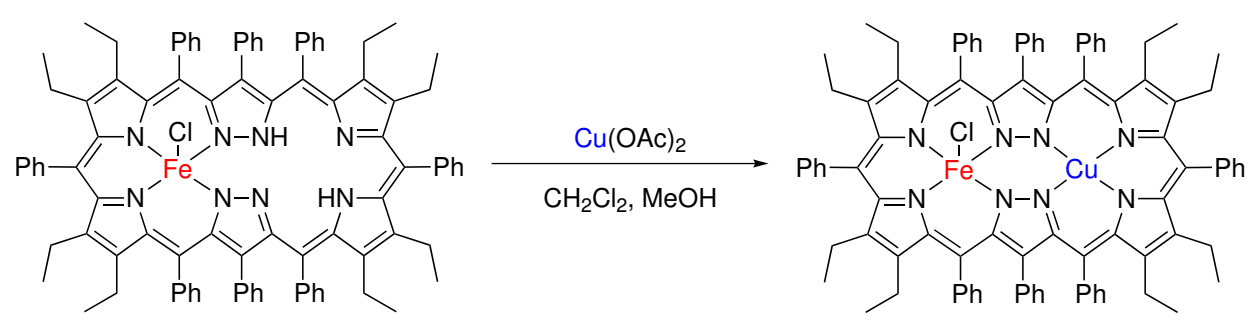

The residue of the synthesis of $\mathbf{L H}_{\mathbf{2}} \mathbf{F e}$ was dissolved in toluene $(10 \mathrm{~mL})$ and added to a solution of copper acetate $(104.6 \mathrm{mg}, 576.0 \mu \mathrm{mol}, 15.0$ eq.) in $\mathrm{MeOH}(25 \mathrm{~mL})$. The reaction mixture was stirred for $30 \mathrm{~min}$ at ambient temperature and the solvent was removed under reduced pressure. The residue was redissolved in $\mathrm{CH}_{2} \mathrm{Cl}_{2}$ and filtered over a plug of basic aluminum oxide to remove $\mathbf{L} \mathbf{C u}_{\mathbf{2}}$. The remaining $\mathbf{L C u F e}$ was eluted with $\mathrm{MeOH}$ and the solvent was removed under reduced pressure. The crude product was purified by column chromatography (silica, $n$-hexane/EtOAc 4:1) and recrystallized from a mixture of $\mathrm{CH}_{2} \mathrm{Cl}_{2}$ and $n$-hexane (1:1) to yield LCuFeCl $(25 \mathrm{mg}, 17.2 \mu \mathrm{mol}$, $45 \%)$.
Empirical Formula:
$\mathrm{C}_{92} \mathrm{H}_{80} \mathrm{~N}_{8} \mathrm{CuFeCl}$
Molecular Weight $(\mathrm{g} / \mathrm{mol})$ :
1452.52 
Yield:

$\mathbf{R}_{f}$ (silica, $n$-hexane/EtOAc 4:1):

HRMS $\left(\mathrm{ESI}^{+}, \mathrm{MeOH}\right): m / z=$ IR $(\mathrm{KBr})$ :
$25 \mathrm{mg}, 45 \%$ (over two steps)

0.47

1415.5149 (calcd. 1415.5150) $[\mathrm{M}]^{+}$.

$\widetilde{v}\left(\mathrm{~cm}^{-1}\right)=3058(\mathrm{~m}), 3020(\mathrm{w}), 2968(\mathrm{~s}), 2927$

$(\mathrm{s}), 2867(\mathrm{~m}), 2146(\mathrm{w}), 1881(\mathrm{w}), 1852(\mathrm{w})$,

$1761(\mathrm{w}), 1727(\mathrm{w}), 1634(\mathrm{~s}), 1558(\mathrm{~m}), 1517$

(w), $1496(\mathrm{~m}), 1427$ (vs), $1381(\mathrm{~m}), 1351$ (w),

$1292(\mathrm{~m}), 1248(\mathrm{~m}), 1184(\mathrm{~m}), 1154(\mathrm{w}), 1110$

(s), $1052(\mathrm{~m}), 1020(\mathrm{~m}), 930(\mathrm{~m}), 893(\mathrm{~m}), 797$

$(\mathrm{s}), 781(\mathrm{~s}), 730(\mathrm{~m}), 693(\mathrm{~s}), 655(\mathrm{w}), 615(\mathrm{w})$, $550(\mathrm{w}), 503(\mathrm{w}), 462(\mathrm{w})$.

388 (72500), 560 (21100), 692 (17300).

\subsubsection{Iron(III) Iron(III) Complex $\mathrm{L}\{\mathrm{FeCl}\}_{2}$}
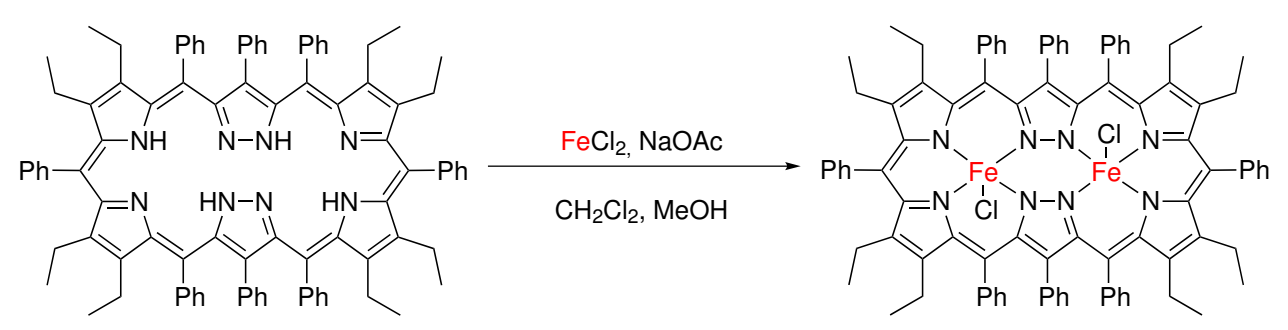

$\mathrm{FeCl}_{2}(117.0 \mathrm{mg}, 923 \mu \mathrm{mol}, 6.0$ eq.) and $\mathrm{NaOAc}(113.5 \mathrm{mg}, 1384 \mu \mathrm{mol}, 9.0$ eq.) were added to a solution of the free base Siamese-twin porphyrin $\mathbf{L H}_{\mathbf{4}}(200 \mathrm{mg}, 154 \mu \mathrm{mol}$, 1.0 eq.) in dichloromethane $(20 \mathrm{~mL})$ and $\mathrm{MeOH}(40 \mathrm{~mL})$. The mixture was stirred for 30 minutes at ambient temperature. After removing of the solvent under reduced pressure, $\mathrm{Et}_{2} \mathrm{O}(20 \mathrm{~mL})$ was added and the suspension was filtered. The solvent was removed under reduced pressure and the residue was redissolved in dichloromethane $(20 \mathrm{~mL})$, washed with brine and dried over sodium sulfate. The raw product was cleaned by size exclusion chromatography (Sephadex ${ }^{\mathrm{TM}}, \mathrm{CH}_{2} \mathrm{Cl}_{2}$ ) and recrystallized by slow evaporation of $n$-heptane into a solution of the raw product in chlorobenzene to yield $70 \mathrm{mg}$ (31\%) $\mathbf{L}\{\mathbf{F e C l}\}_{\mathbf{2}}$. Crystals suitable for X-Ray crystallography were obtained by slow evaporation of a saturated solution of $\mathbf{L}\{\mathbf{F e C l}\}_{2}$ in benzene into toluene.

Empirical Formula:

Molecular Weight $(\mathrm{g} / \mathrm{mol})$ :

HRMS $\left(\mathrm{ESI}^{+}, \mathrm{MeOH}\right): m / z=$
$\mathrm{C}_{92} \mathrm{H}_{80} \mathrm{~N}_{8} \mathrm{Fe}_{2} \mathrm{Cl}_{2}$

1480.27

1439.5375 (calcd. 1439.5386) $\left[\mathrm{M}+\mathrm{CH}_{3} \mathrm{O}\right]^{+}$, 704.2602 (calcd. 704.2601) $[\mathrm{M}]^{2+}$. 
IR $(\mathrm{KBr})$ :

UV-vis/nm $\left(\epsilon / \mathrm{M}^{-1} \mathrm{~cm}^{-1}\right)$ : $\widetilde{v}\left(\mathrm{~cm}^{-1}\right)=3055(\mathrm{w}), 3025(\mathrm{w}), 2971(\mathrm{~m}), 2929$ (m), $2871(\mathrm{~m}), 1669(\mathrm{w}), 1642(\mathrm{w}), 1598(\mathrm{w})$, $1569(\mathrm{w}), 1548(\mathrm{w}), 1519(\mathrm{w}), 1447(\mathrm{w}), 1426$ (vs), $1377(\mathrm{w}), 1347(\mathrm{w}), 1314(\mathrm{w}), 1288(\mathrm{~m})$, $1245(\mathrm{~m}), 1177(\mathrm{~m}), 1156(\mathrm{w}), 1111(\mathrm{~m}), 1054$ (m), $1005(\mathrm{~m}), 931(\mathrm{w}), 895(\mathrm{w}), 844(\mathrm{w}), 813$ $(\mathrm{w}), 771(\mathrm{~m}), 750(\mathrm{~m}), 696(\mathrm{~s}), 567(\mathrm{w}), 544(\mathrm{w})$, $498(\mathrm{w}), 461(\mathrm{w}), 398(\mathrm{w}), 376(\mathrm{w}), 360(\mathrm{w})$. 382 (67400), 535 (20400), 667 (11300).

\subsubsection{Nickel(II) Cobalt(III) Complex $\mathrm{LNiCo}^{+}$}
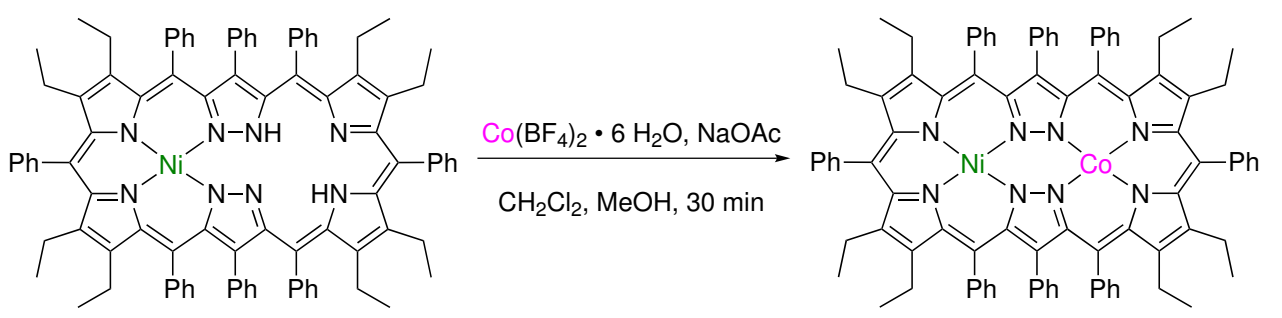

$\mathrm{Co}\left(\mathrm{BF}_{4}\right)_{2} \cdot 6 \mathrm{H}_{2} \mathrm{O}$ (45.2 mg, $132.7 \mu \mathrm{mol}, 9.0$ eq.) and $\mathrm{NaOAc}(10.9 \mathrm{mg}, 132.7 \mu \mathrm{mol}$, 9.0 eq.) were added to a solution of the mononickel complex $\mathbf{L H}_{\mathbf{2}} \mathbf{N i}(20 \mathrm{mg}, 14.7 \mu \mathrm{mol}$, 1.0 eq.) in $\mathrm{CH}_{2} \mathrm{Cl}_{2}(2 \mathrm{~mL})$ and $\mathrm{MeOH}(4 \mathrm{~mL})$ under inert conditions. The reaction mixture was stirred at ambient temperature for 30 minutes. The solvent was removed under reduced pressure and the residue was filtered over a plug of basic aluminum oxide. The solvent was removed under reduced pressure, yielding $\mathbf{L N i C o}$ as a red solid (8 mg, $39 \%)$

\section{Empirical Formula:}

Molecular Weight $(\mathrm{g} / \mathrm{mol})$ :

HRMS $\left(\mathrm{ESI}^{+}, \mathrm{MeOH}\right): m / z=$
$\mathrm{C}_{92} \mathrm{H}_{80} \mathrm{~N}_{8} \mathrm{NiCo}$

1415.30

1413.5186 (calcd. 1413.5171) $[\mathrm{M}]^{+}$. 


\subsubsection{Cobalt(III) Cobalt(III) Complex $\mathrm{LCo}_{2}{ }^{2+}$}
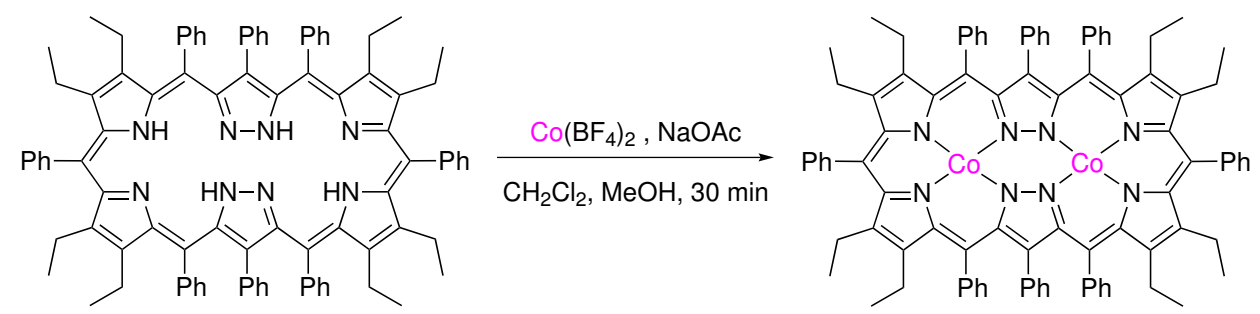

$\mathrm{Co}\left(\mathrm{BF}_{4}\right)_{2} \cdot 6 \mathrm{H}_{2} \mathrm{O}$ (47.1 mg, $138.4 \mu \mathrm{mol}, 9.0$ eq.) and NaOAc (11.4 mg, $138.4 \mu \mathrm{mol}$, 9.0 eq.) were added to a solution of the free base Siamese-twin porphyrin $\mathbf{L H}_{\mathbf{4}}(20 \mathrm{mg}$, $15.4 \mu \mathrm{mol}, 1.0$ eq. $)$ in $\mathrm{CH}_{2} \mathrm{Cl}_{2}(2 \mathrm{~mL})$ and $\mathrm{MeOH}(4 \mathrm{~mL})$ under inert conditions. The reaction mixture was stirred at ambient temperature for 30 minutes. The solvent was removed under reduced pressure and the residue was filtered over a plug of basic aluminum oxide. The solvent was removed under reduced pressure, yielding $\mathbf{L C o}_{2}$ as a red solid that immediately started to decompose.

Empirical Formula:

Molecular Weight $(\mathrm{g} / \mathrm{mol})$ :

HRMS $\left(\mathrm{ESI}^{+}, \mathrm{MeOH}\right): m / z=$
$\mathrm{C}_{92} \mathrm{H}_{80} \mathrm{~N}_{8} \mathrm{Co}_{2}$

1415.54

1414.5164 (calcd. 1414.5164) [M] ${ }^{+}$. 


\section{Appendix}

\section{Crystallographic Data}

One $\mathrm{CH}_{2} \mathrm{Cl}_{2}$ in ${ }^{p \mathbf{M e}} \mathbf{L H}_{4}$ was found to be disordered about two positions (occupancy factors: $0.70(1) / 0.30(1)$ ). SAME and RIGU restraints were applied to model the disorder. The unit cells of ${ }^{p \mathbf{M e}} \mathbf{L H}_{4}$ and ${ }^{p \mathrm{~F}} \mathbf{L H}_{4}$ contain highly disordered $\mathrm{CH}_{2} \mathrm{Cl}_{2}$ molecules (probably four in case of ${ }^{p \mathbf{M e}} \mathbf{L H}_{4}$ and five in case of ${ }^{p \mathbf{F}} \mathbf{L} \mathbf{H}_{\mathbf{4}}$ ) for which no satisfactory model for a disorder could be found. The solvent contribution to the structure factors was calculated with PLATON SQUEEZE ${ }^{[191]}$ and the resulting .fab file was processed with SHELXL ${ }^{[188]}$ using the ABIN instruction. The empirical formula and derived values are in accordance with the calculated cell content. For Faceindexed absorption corrections for ${ }^{p \mathbf{M e}} \mathbf{L H}_{4}$ were performed numerically with the program X-RED (X-RED; STOE \& CIE GmbH: Darmstadt, Germany, 2002.). CCDC1484865 and -1484866 contain the supplementary crystallographic data. These data can be obtained free of charge from The Cambridge Crystallographic Data Centre via http://www.ccdc.cam.ac.uk/data_request/cif.

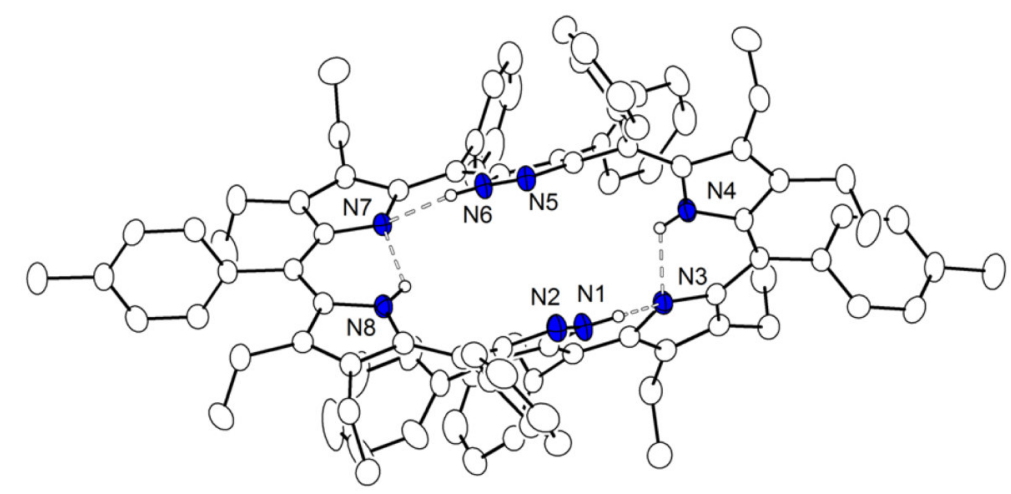

Figure A1: Plot of the molecular structure of ${ }^{p \mathrm{Me}} \mathbf{L H}_{4}$ (50\% probability thermal ellipsoids; most hydrogen atoms and solvent molecules omitted for clarity). Selected bond lengths $[\AA]$ and angles $\left[{ }^{\circ}\right]: \mathrm{N} 1 \cdots \mathrm{N} 32.627(2), \mathrm{N} 4 \cdots \mathrm{N} 32.636(2), \mathrm{N} 6 \cdots \mathrm{N} 72.681(2)$,

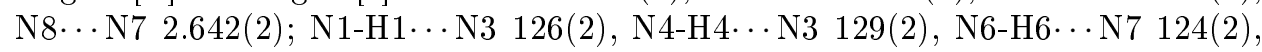
N8-H8 $\cdots$ N7 129(2). 


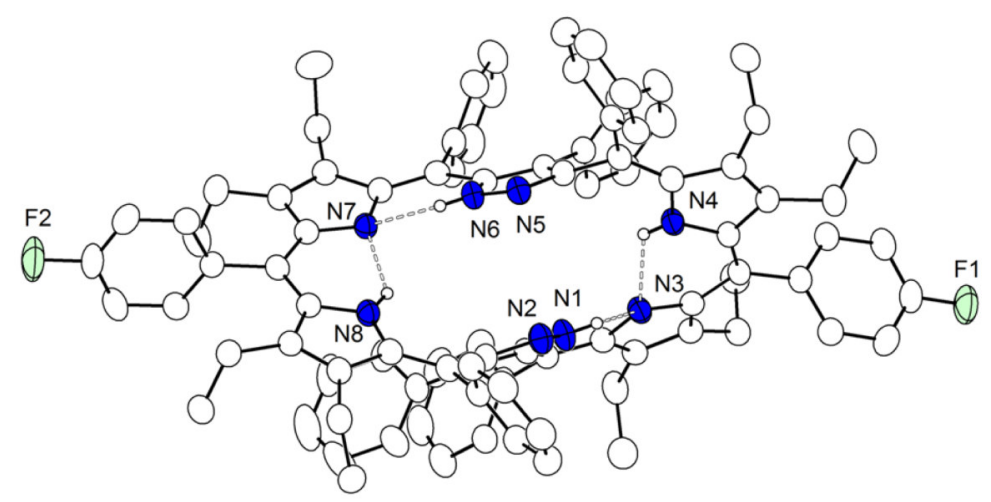

Figure A2: Plot of the molecular structure of ${ }^{p \mathrm{~F}} \mathbf{L H}_{4}$ (50\% probability thermal ellipsoids; most hydrogen atoms omitted for clarity). Selected bond lengths $[\AA]$ and angles $\left[{ }^{\circ}\right.$ ]: N1 $\cdots \mathrm{N} 32.607(3), \mathrm{N} 4 \cdots \mathrm{N} 32.08(4), \mathrm{N} 6 \cdots \mathrm{N} 72.694(3), \mathrm{N} 8 \cdots \mathrm{N} 72.630(3)$; N1-

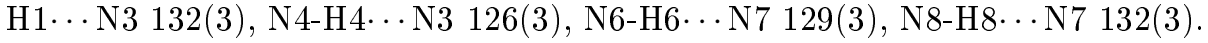

\begin{tabular}{|c|c|c|}
\hline compound & ${ }^{p \mathrm{Me}} \mathrm{LH}_{4}$ & ${ }^{p \mathrm{~F}} \mathbf{L H}_{4}$ \\
\hline empirical formula & $\mathrm{C}_{96} \mathrm{H}_{92} \mathrm{Cl}_{4} \mathrm{~N}_{8}$ & $\mathrm{C}_{93} \mathrm{H}_{84} \mathrm{Cl}_{2} \mathrm{~F}_{2} \mathrm{~N}_{8}$ \\
\hline formula weight & 1499.57 & 1422.58 \\
\hline$T[\mathrm{~K}]$ & $133(2)$ & $133(2)$ \\
\hline crystal size $\left[\mathrm{mm}^{3}\right]$ & $0.50 \times 0.32 \times 0.29$ & $0.50 \times 0.49 \times 0.34$ \\
\hline crystal system & monoclinic & monoclinic \\
\hline space group & $\mathrm{P} 2{ }_{1} / \mathrm{n}$ & $\mathrm{P} 2_{1} / \mathrm{n}$ \\
\hline$a[\AA]$ & $13.5344(3)$ & $13.0633(3)$ \\
\hline$b[\AA]$ & $29.1838(5)$ & $28.9457(7)$ \\
\hline$c[\AA]$ & $24.3828(6)$ & $24.9657(5)$ \\
\hline$\alpha\left[^{\circ}\right]$ & 90 & 90 \\
\hline$\beta\left[^{\circ}\right]$ & $102.500(2)$ & $104.034(2)$ \\
\hline$\gamma\left[^{\circ}\right]$ & 90 & 90 \\
\hline$V\left[\AA^{3}\right]$ & $9402.6(4)$ & $9158.4(4)$ \\
\hline$Z$ & 4 & 4 \\
\hline$\rho\left[\mathrm{g} / \mathrm{cm}^{3}\right]$ & 1.059 & 1.032 \\
\hline$F(000)$ & 3168 & 3000 \\
\hline$\mu\left[\mathrm{mm}^{-1}\right]$ & 0.171 & 0.120 \\
\hline$T_{\min } / T_{\max }$ & $0.6111 / 0.9378$ & - \\
\hline$\theta$-range $\left[^{\circ}\right]$ & $1.395-25.673$ & $1.407-25.636$ \\
\hline$h k l$-range & $\pm 16,-32-35, \pm 29$ & $\pm 15, \pm 35, \pm 30$ \\
\hline measured refl. & 99795 & 106084 \\
\hline unique refl. [Rint] & $17719[0.0593]$ & $17249[0.0574]$ \\
\hline observed refl. $(I>2 \sigma(I))$ & 14017 & 14282 \\
\hline data / restraints / param. & $17719 / 27 / 1027$ & $17249 / 0 / 970$ \\
\hline goodness-of-fit $\left(F^{2}\right)$ & 1.029 & 1.094 \\
\hline$R 1, w R 2(I>2 \sigma(I))$ & $0.0547,0.1236$ & $0.0632,0.1497$ \\
\hline$R 1, w R 2$ (all data) & $0.0717,0.1313$ & $0.0746,0.1547$ \\
\hline resid. el. dens. $\left[\mathrm{e} / \AA^{3}\right]$ & $-0.504 / 0.463$ & $-0.388 / 0.233$ \\
\hline
\end{tabular}


In $\mathbf{L N i F e C l}$ the $\mathrm{FeCl}$ moiety and the nickel atom are disordered about the two coordination sites of the ligand in a 1:1 ratio (1/2 occupancy) along with a DMF molecule $\left(\mathrm{d}\left(\mathrm{O}^{\mathrm{DMF}}-\mathrm{Fe}\right)=3.13 \AA ; \mathrm{d}\left(\mathrm{O}^{\mathrm{DMF}}-\mathrm{Ni}\right)=2.64 \AA\right)$ and acetone and water $\left(\mathrm{O}^{\mathrm{w}}\right.$ and $\mathrm{O}^{\text {acetone }}$ not in a coordinating range). DMF and acetone were refined at $1 / 2$, water at $1 / 4$ occupancy. SIMU, DELU, ISOR, DFIX, SADI, FLAT restraints and EADP constraints were applied to model the disordered parts. Face-indexed absorption corrections were performed numerically with the program X-RED (X-RED; STOE \& CIE GmbH: Darmstadt, Germany, 2002.).

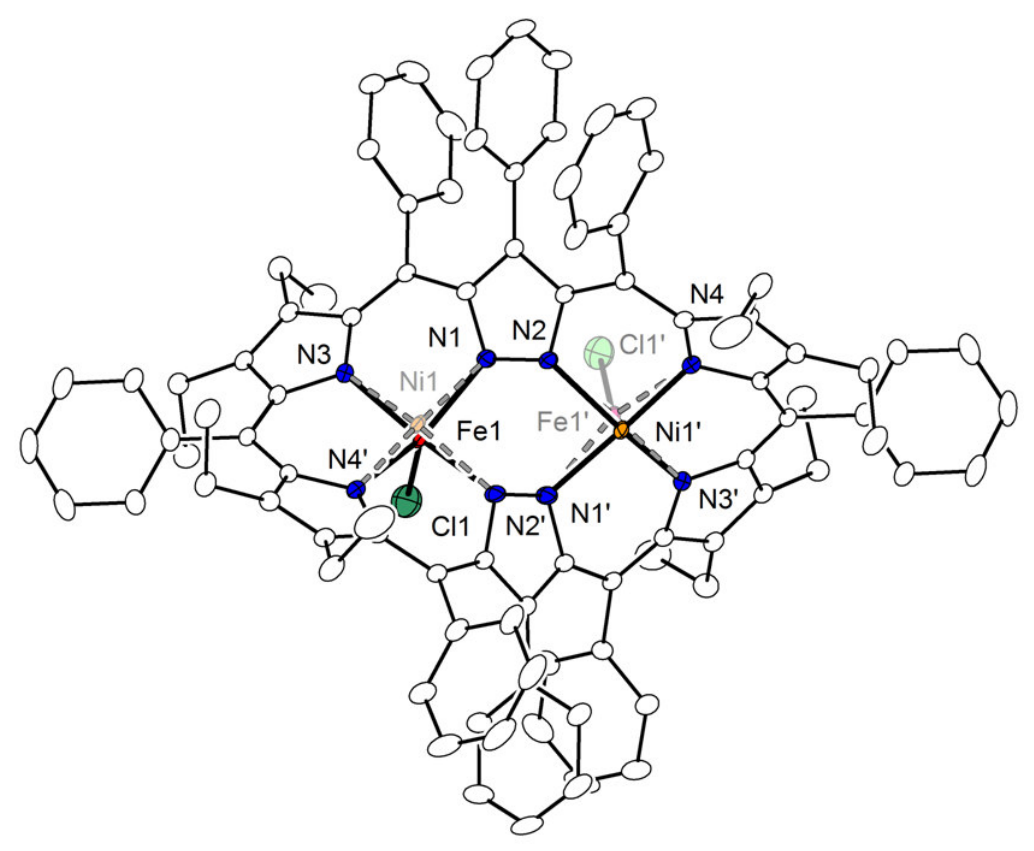

Figure A3: Plot of the molecular structure of om 49 (30\% probability thermal ellipsoids; hydrogen atoms, most disordered parts and solvent molecules omitted for clarity) emphasizing the disorder of the nickel atom and the FeCl moiety. Selected bond lengths $[\AA]$

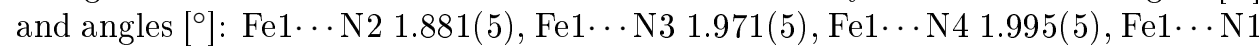
$2.017(5), \mathrm{Fe} 1 \cdots \mathrm{Cl} 12.446(4), \mathrm{Ni} 1 \cdots \mathrm{N} 31.873(5), \mathrm{Ni} 1 \cdots \mathrm{N} 41.910(5), \mathrm{Ni} 1 \cdots \mathrm{N} 1$

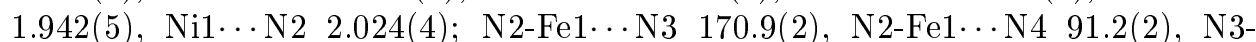

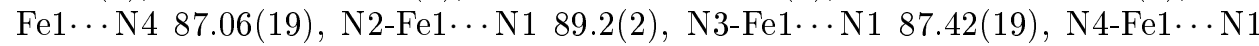

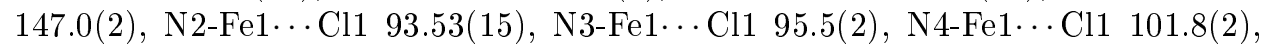

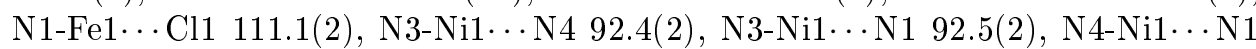

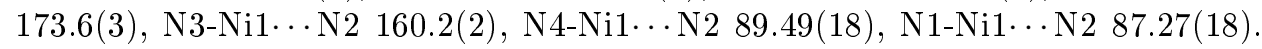
Symmetry transformation used to generate equivalent atoms: (') 1-x, y, 1/2-z. 
In $\mathbf{L}\{\mathbf{F e C l}\}_{2}$ one phenyl group $(\mathrm{C} 9 \mathrm{~A} / \mathrm{B}-\mathrm{C} 14 \mathrm{~A} / \mathrm{B} ;$ occupancy factors = $0.467(12) / 0.533(12)$ ) and one ethyl group (C19A/B \& C20A/B; occupancy factors $=0.453(12) / 0.547(12))$ of the STP were found to be disordered as well as one of the solvent benzene molecules. The latter one was found to be disordered about a center of inversion and was refined at half occupancy. AFIX 66 (for the phenyl rings), SADI, SIMU, DELU, ISOR, DFIX restraints and EADP constraints were applied to model the disorder. Face-indexed absorption corrections were performed numerically with the program X-RED (X-RED; STOE \& CIE GmbH: Darmstadt, Germany, 2002.).

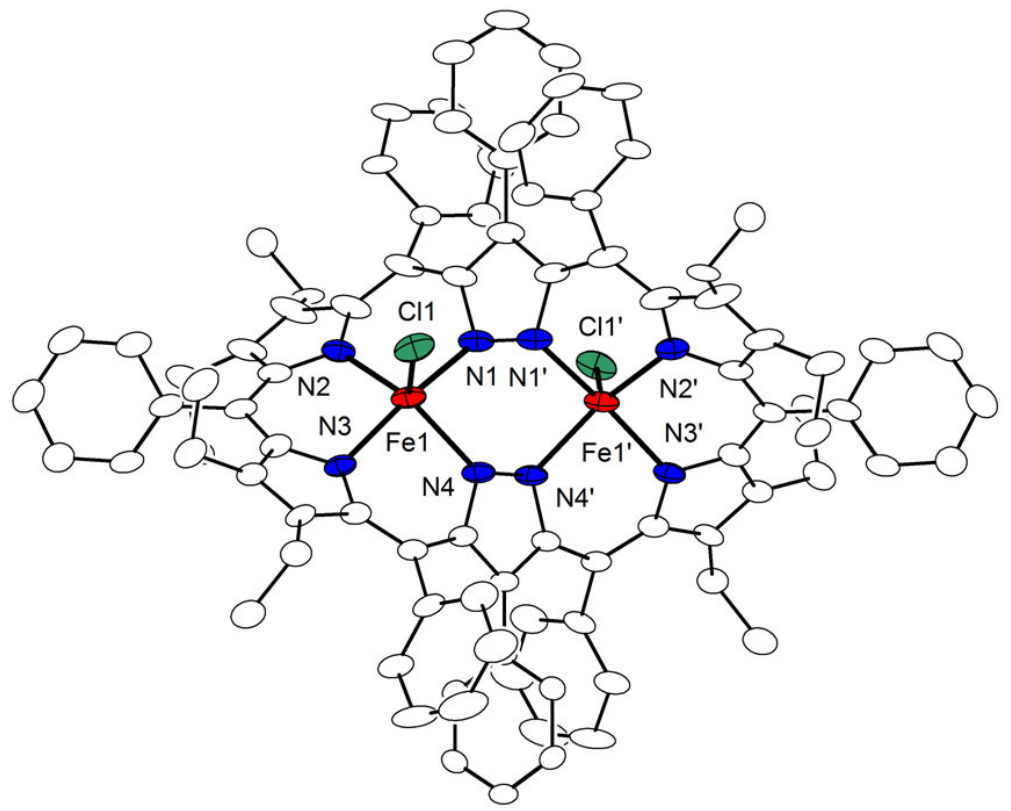

Figure A4: Plot of the molecular structure of $\mathbf{L}\{\mathbf{F e C l}\}_{2}$ (30\% probability thermal ellipsoids; hydrogen atoms, disorder and solvent molecules omitted for clarity). Se-

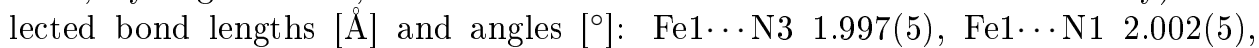
$\mathrm{Fe} 1 \cdots \mathrm{N} 2 \quad 2.036(5), \quad \mathrm{Fe} 1 \cdots \mathrm{N} 4$ 2.061(4), $\mathrm{Fe} 1 \cdots \mathrm{Cl} 12.2732(18) ; \quad \mathrm{N} 3-\mathrm{Fe} 1 \cdots \mathrm{N} 1$

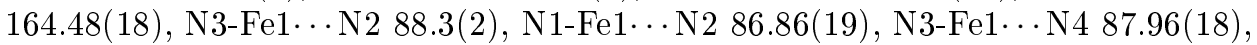

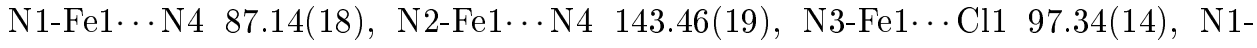

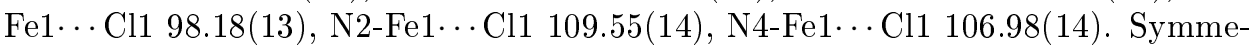
try transformation used to generate equivalent atoms: (') 1-x, y, 3/2-z. 


\begin{tabular}{|c|c|c|}
\hline compound & LNiFeCl & $\mathrm{L}\{\mathrm{FeCl}\}_{2}$ \\
\hline empirical formula & $\mathrm{C}_{98} \mathrm{H}_{93} \mathrm{~N}_{9} \mathrm{NiFeClO}_{2.5}$ & $\mathrm{C}_{110} \mathrm{H}_{98} \mathrm{~N}_{8} \mathrm{Fe}_{2} \mathrm{Cl}_{2}$ \\
\hline formula weight & 1586.82 & 1714.56 \\
\hline$T[\mathrm{~K}]$ & $133(2)$ & $133(2)$ \\
\hline crystal size $\left[\mathrm{mm}^{3}\right]$ & $0.38 \times 0.24 \times 0.18$ & $0.26 \times 0.19 \times 0.13$ \\
\hline crystal system & monoclinic & monoclinic \\
\hline space group & $\mathrm{C} 2 / \mathrm{c}$ & $\mathrm{C} 2 / \mathrm{c}$ \\
\hline$a[\AA]$ & $35.0406(11)$ & $19.2994(14)$ \\
\hline$b[\AA]$ & $11.9424(2)$ & $22.3959(18)$ \\
\hline$c[\AA]$ & $25.6270(8)$ & $22.4844(16)$ \\
\hline$\alpha\left[^{\circ}\right]$ & 90 & 90 \\
\hline$\beta\left[^{\circ}\right]$ & $127.579(2)$ & $109.565(5)$ \\
\hline$\gamma\left[^{\circ}\right]$ & 90 & 90 \\
\hline$V\left[\AA^{3}\right]$ & $8499.0(4)$ & $9157.3(12)$ \\
\hline$Z$ & 4 & 4 \\
\hline$\rho\left[\mathrm{g} / \mathrm{cm}^{3}\right]$ & 1.240 & 1.244 \\
\hline$F(000)$ & 3340 & 3600 \\
\hline$\mu\left[\mathrm{mm}^{-1}\right]$ & 0.479 & 0.429 \\
\hline$T_{\min } / T_{\max }$ & $0.8468 / 0.9368$ & $0.7590 / 0.9321$ \\
\hline$\theta$-range $\left[{ }^{\circ}\right]$ & $1.467-25.667$ & $1.442-25.780$ \\
\hline$h k l$-range & $\pm 42, \pm 14,-31-28$ & $\pm 23, \pm 27,-26-27$ \\
\hline measured refl. & 52231 & 49488 \\
\hline unique refl. [Rint] & $8018[0.0610]$ & $8729[0.1606]$ \\
\hline observed refl. $(I>2 \sigma(I))$ & 6064 & 3994 \\
\hline data / restraints / param. & $8018 / 128 / 566$ & $8729 / 86 / 573$ \\
\hline goodness-of-fit $\left(F^{2}\right)$ & 1.068 & 0.970 \\
\hline$R 1, w R 2(I>2 \sigma(I))$ & $0.0742,0.1957$ & $0.0859,0.1672$ \\
\hline$R 1, w R 2$ (all data) & $0.0993,0.2116$ & $0.1926,0.2074$ \\
\hline resid. el. dens. $\left[\mathrm{e} / \AA^{3}\right]$ & $-0.666 / 1.064$ & $-0.248 / 1.188$ \\
\hline
\end{tabular}




\section{Additional Spectroscopic Data of ${ }^{\mathrm{X}} \mathrm{LH}_{4}$}

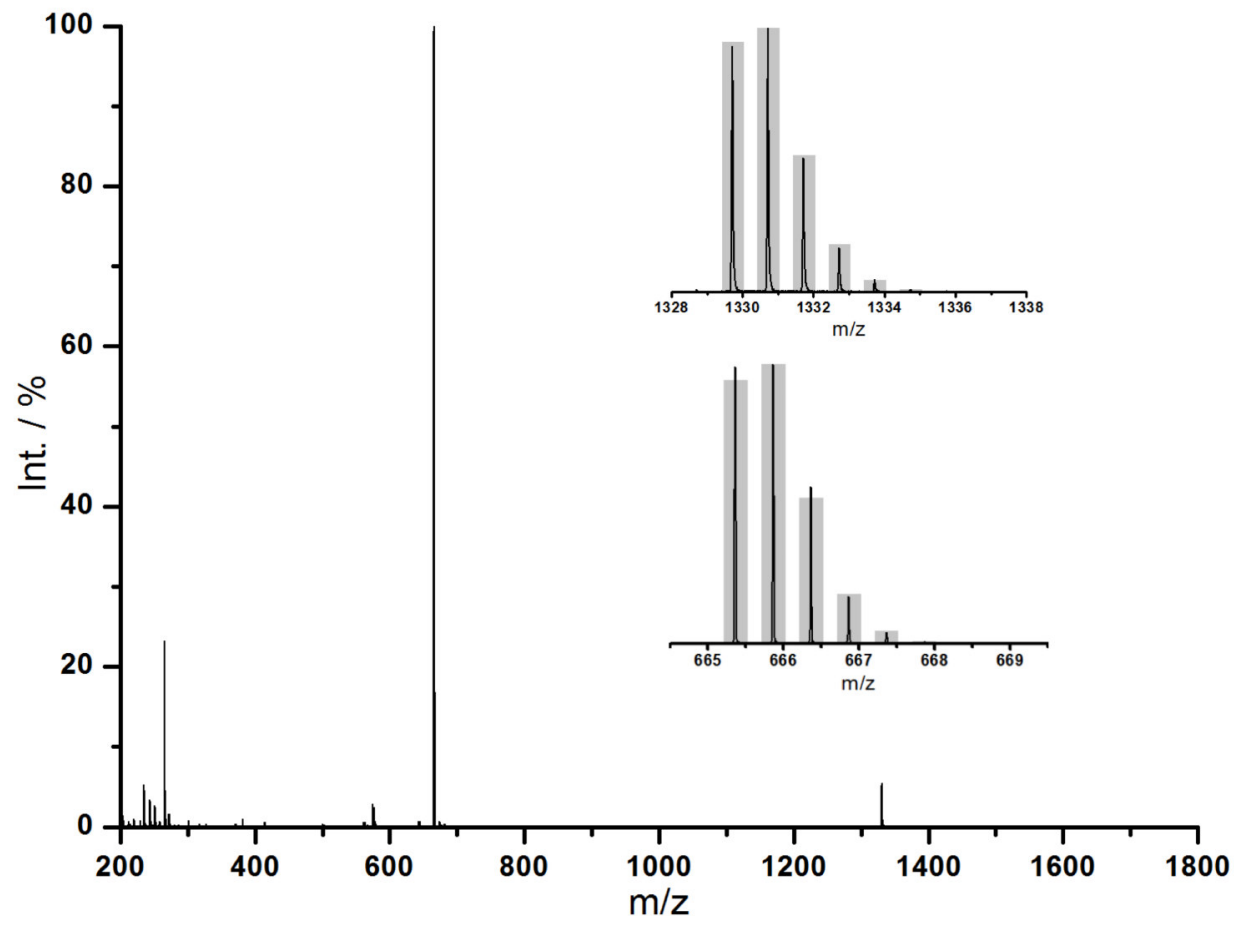

Figure A5: HRMS ESI ${ }^{+}$spectrum of ${ }^{\boldsymbol{p} \mathbf{M e}} \mathbf{L H}_{\mathbf{4}}\left(\mathrm{m} / \mathrm{z}=1329.7217(\right.$ calcd. 1329.7205$)[\mathrm{M}+\mathrm{H}]^{+}$, 665.3634 (calcd. 665.3639) $[\mathrm{M}+2 \mathrm{H}]^{2+}$ ) in MeOH. Inset: Comparison of the measured (black) and calculated isotopic pattern (grey bars).

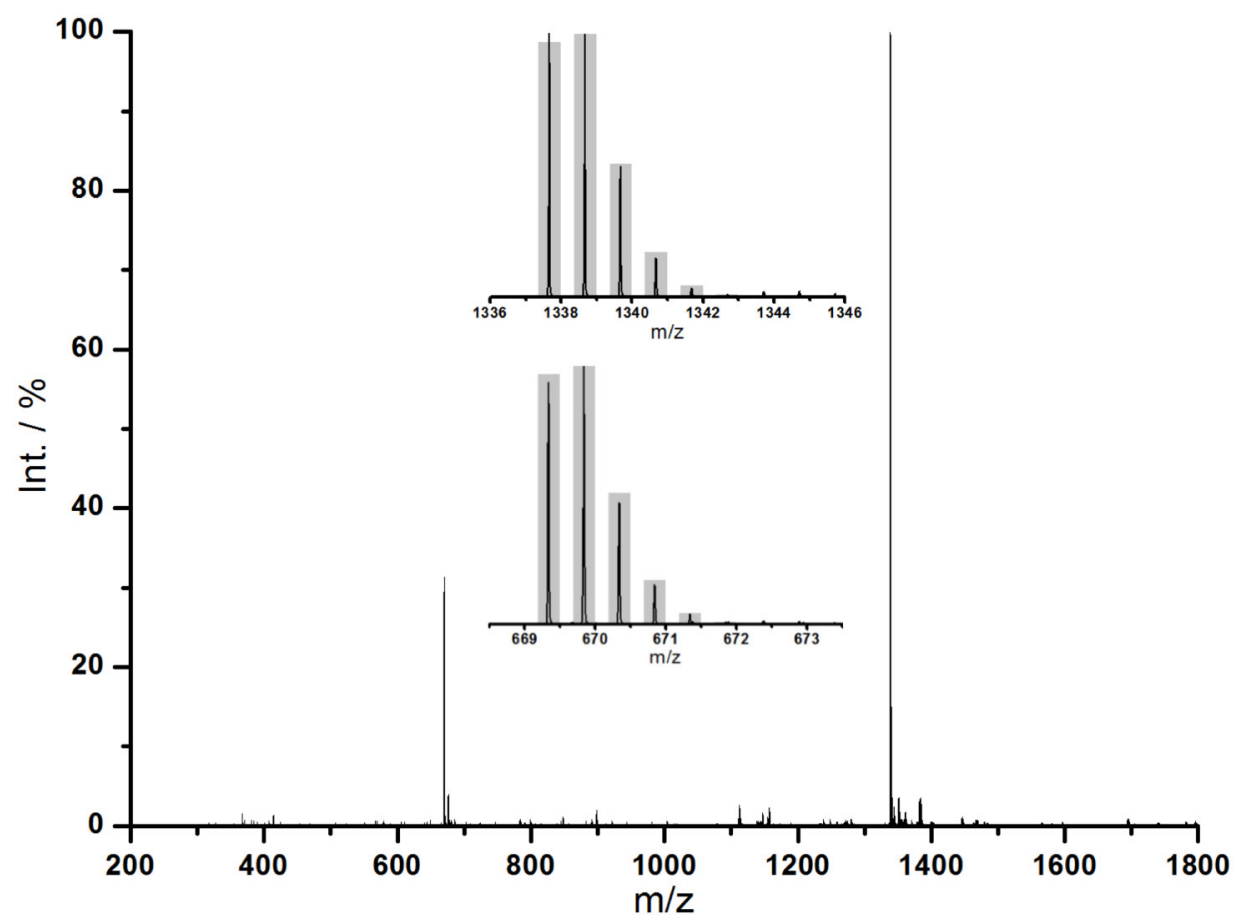

Figure A6: HRMS ESI ${ }^{+}$spectrum of ${ }^{\boldsymbol{p} \mathbf{F}} \mathbf{L H}_{\mathbf{4}}\left(\mathrm{m} / \mathrm{z}=1337.6704\right.$ (calcd. 1337.6703) $[\mathrm{M}+\mathrm{H}]^{+}$, 669.3384 (calcd. 669.3388) $[\mathrm{M}+2 \mathrm{H}]^{2+}$ ) in MeOH. Inset: Comparison of the measured (black) and calculated isotopic pattern (grey bars). 


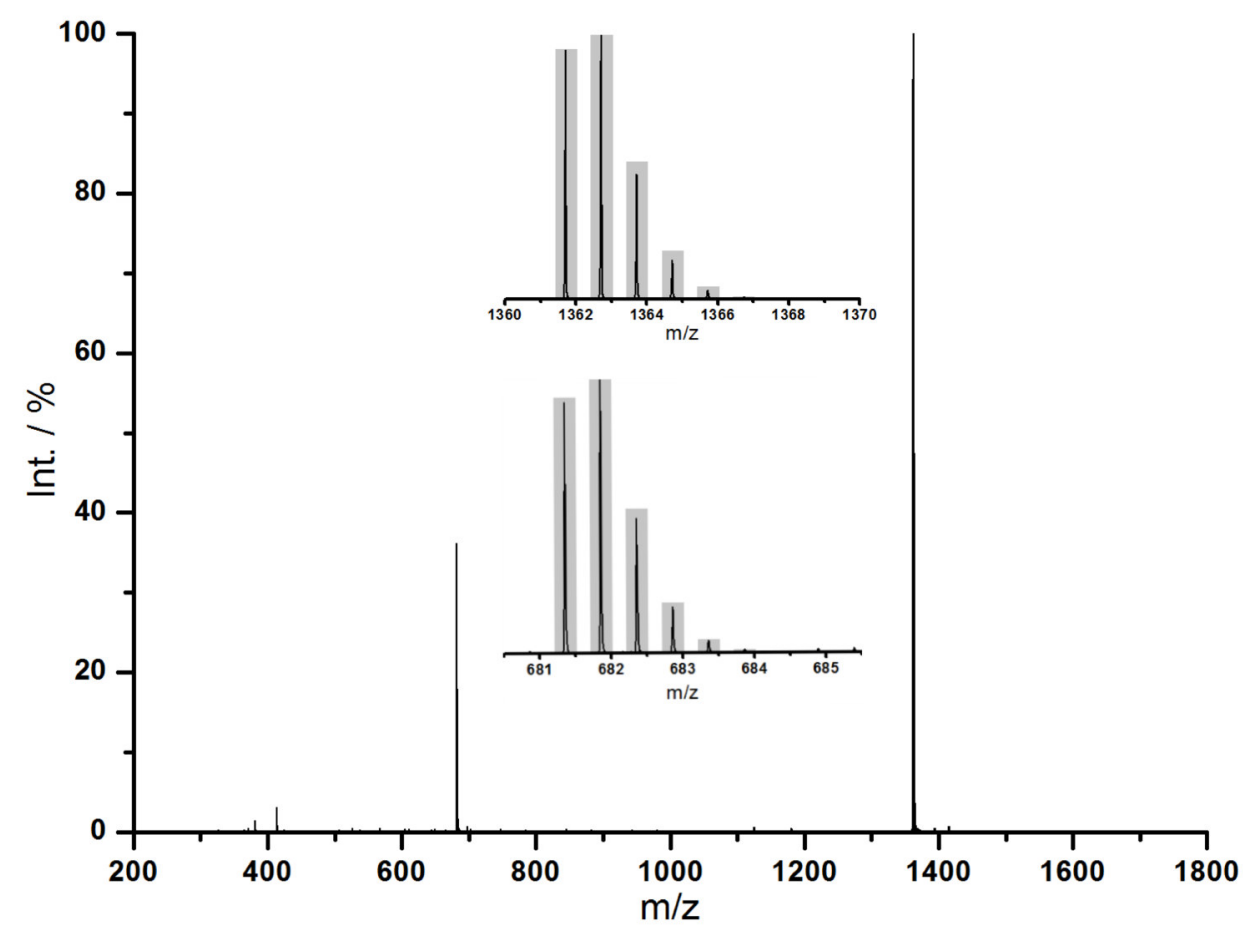

Figure A7: $\mathrm{HRMS} \mathrm{ESI}^{+}$spectrum of ${ }^{\boldsymbol{p} \mathrm{MeO}} \mathbf{L H}_{\mathbf{4}}\left(\mathrm{m} / \mathrm{z}=1361.7104\left(\right.\right.$ calcd. 1361.7103) $[\mathrm{M}+\mathrm{H}]^{+}$, 681.3582 (calcd. 681.3588) $[\mathrm{M}+2 \mathrm{H}]^{2+}$ ) in $\mathrm{MeOH}$. Inset: Comparison of the measured (black) and calculated isotopic pattern (grey bars).

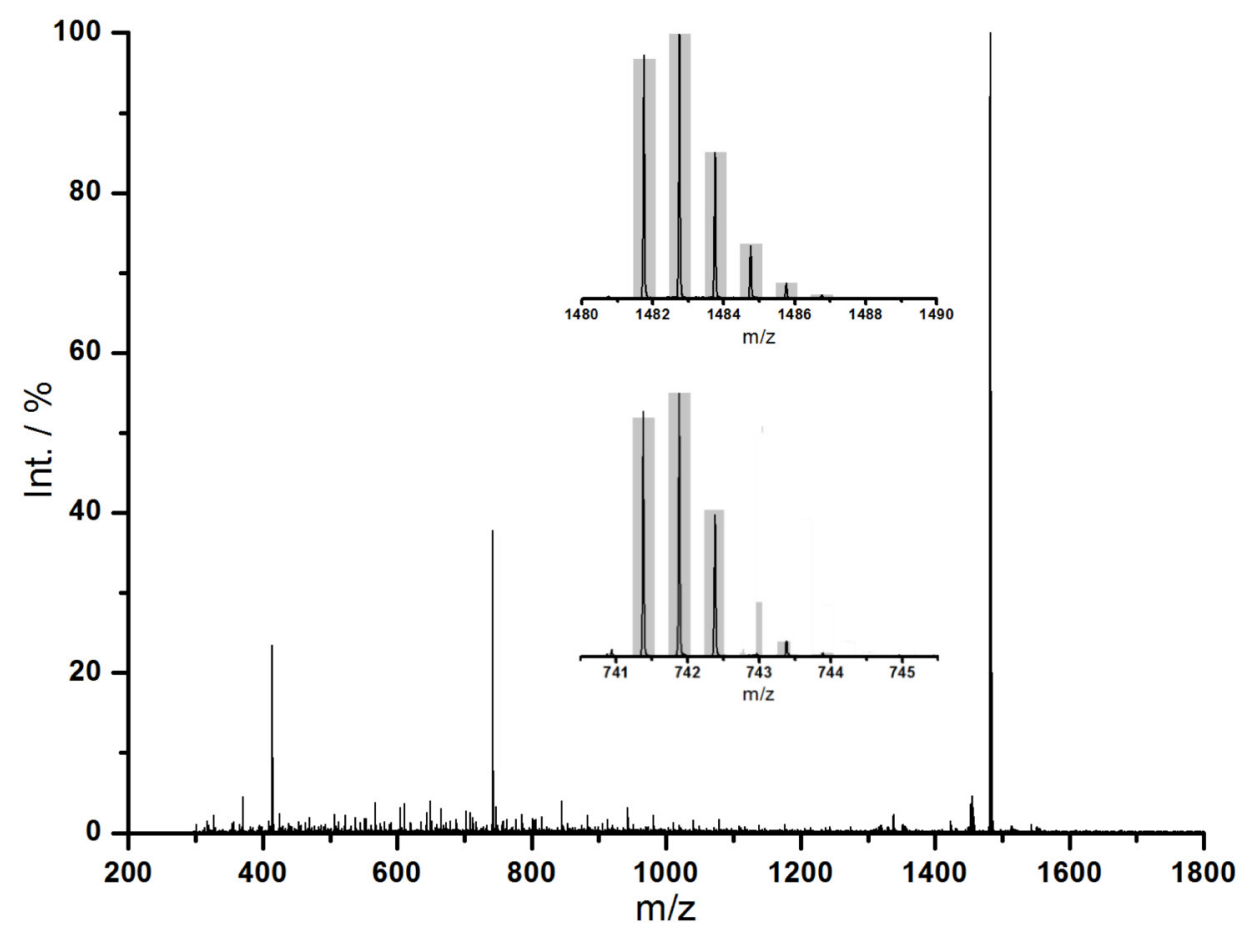

Figure A8: HRMS ESI ${ }^{+}$spectrum of ${ }^{\mathrm{MeO}} \mathbf{L H}_{4}\left(\mathrm{~m} / \mathrm{z}=1481.7526\right.$ (calcd. 1481.7526) $[\mathrm{M}+\mathrm{H}]^{+}$, 741.3794 (calcd. 741.3799) $[\mathrm{M}+2 \mathrm{H}]^{+}$) in $\mathrm{MeOH}$. Inset: Comparison of the measured (black) and calculated isotopic pattern (grey bars). 


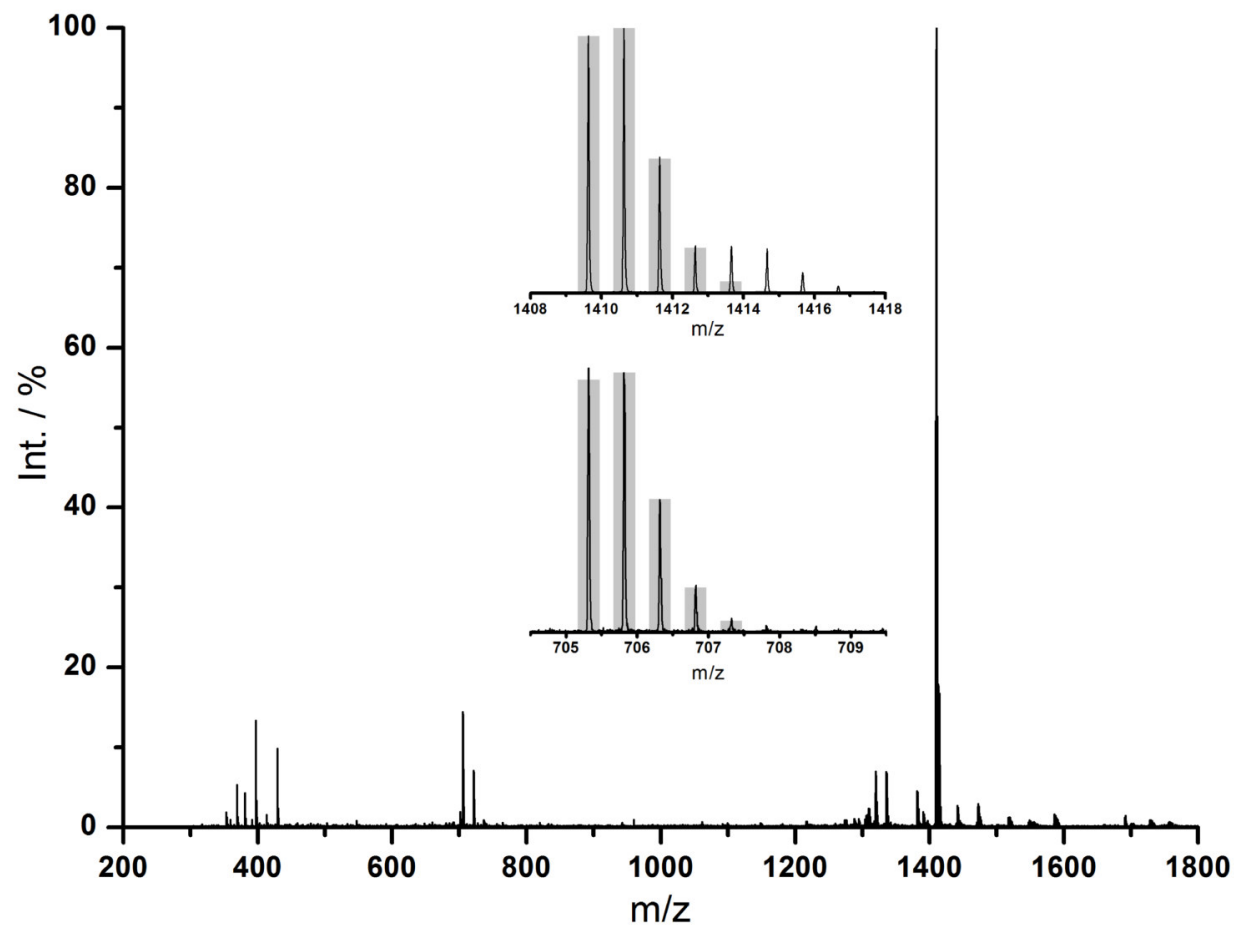

Figure A9: HRMS ESI ${ }^{+}$spectrum of ${ }^{\text {tri-F }} \mathbf{L H}_{4}\left(\mathrm{~m} / \mathrm{z}=1409.6327\right.$ (calcd. 1409.6326) $[\mathrm{M}+\mathrm{H}]^{+}$, 705.3198 (calcd. 705.3200) $[\mathrm{M}+2 \mathrm{H}]^{2+}$ ) in $\mathrm{MeOH}$. Inset: Comparison of the measured (black) and calculated isotopic pattern (grey bars).

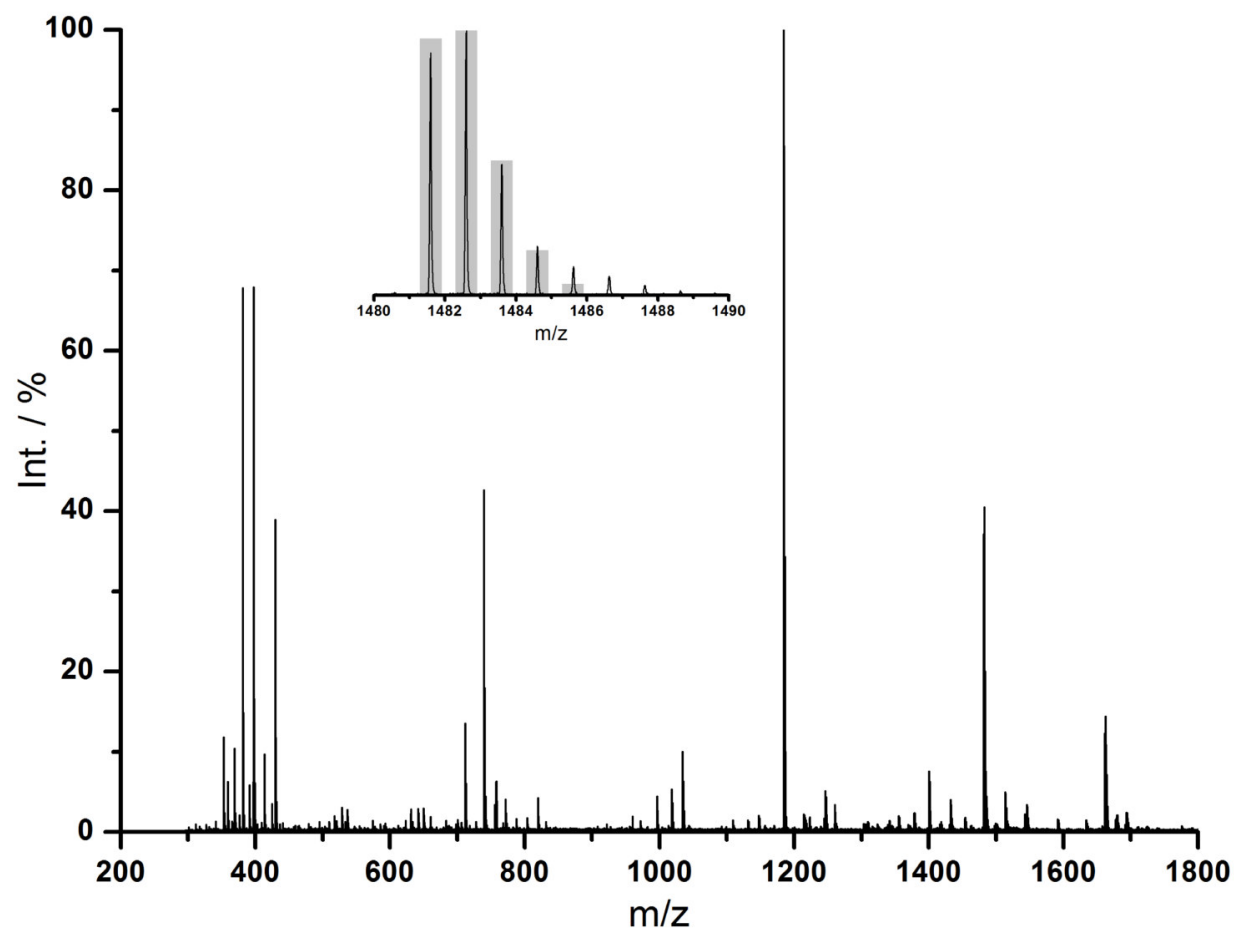

Figure A10: HRMS ESI ${ }^{+}$spectrum of ${ }^{p e n t a-}{ }^{p} \mathbf{L H}_{4}(\mathrm{~m} / \mathrm{z}=1481.5955$ (calcd. 1481.5950) $[\mathrm{M}+\mathrm{H}]^{+}, 741.3005$ (calcd. 741.3011) $[\mathrm{M}+2 \mathrm{H}]^{2+}$ ) in MeOH. Inset: Comparison of the measured (black) and calculated isotopic pattern (grey bars). 


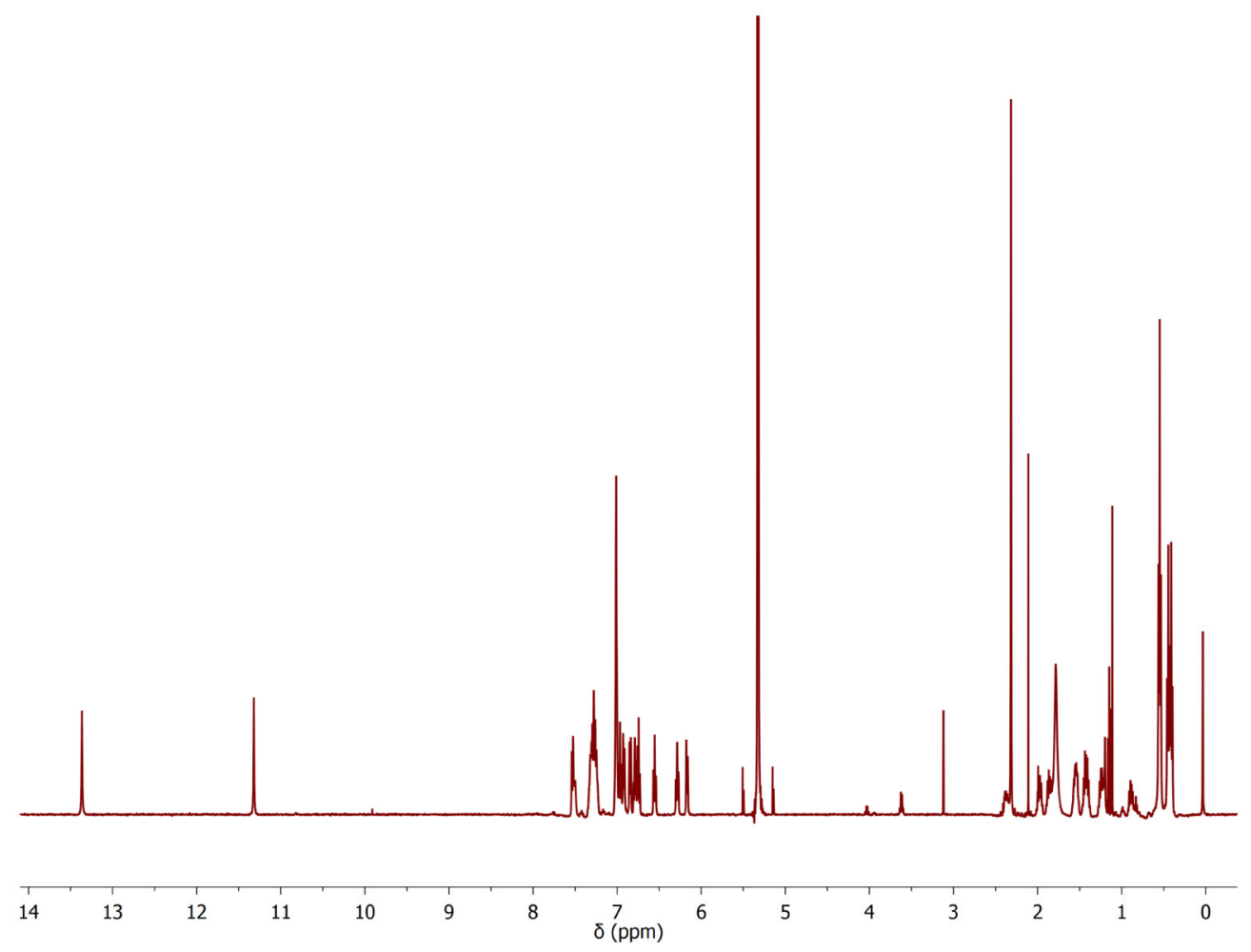

Figure A11: ${ }^{1} \mathrm{H}$ NMR spectrum $\left(500 \mathrm{MHz}, \mathrm{CD}_{2} \mathrm{Cl}_{2}, 238 \mathrm{~K}\right)$ of ${ }^{\boldsymbol{p}}{ }^{\mathrm{Me}} \mathbf{L H}_{\mathbf{4}}$.

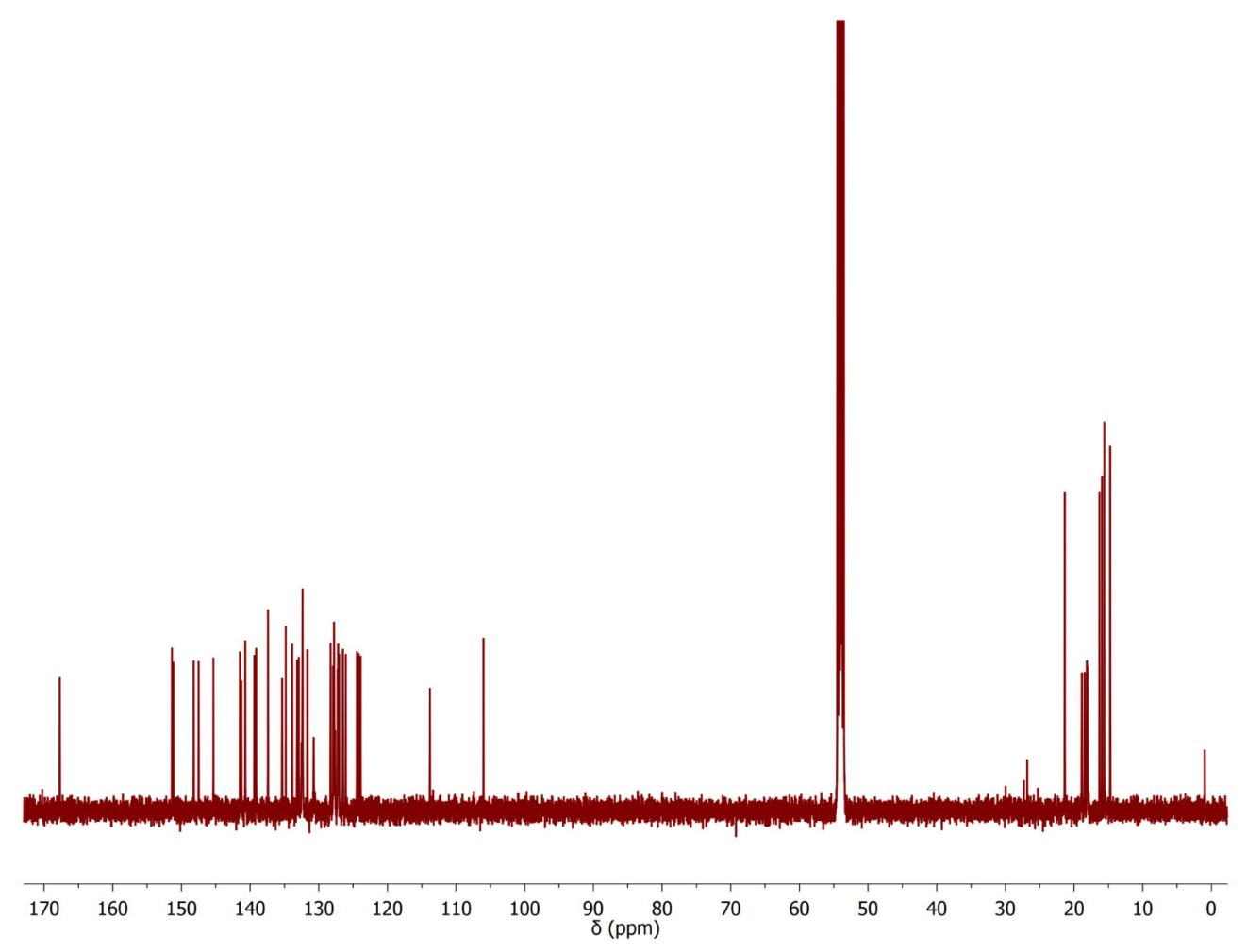

Figure A12: ${ }^{13} \mathrm{C}$ NMR spectrum $\left(125 \mathrm{MHz}, \mathrm{CD}_{2} \mathrm{Cl}_{2}, 238 \mathrm{~K}\right)$ of ${ }^{\boldsymbol{p}} \mathrm{Me}^{\mathrm{e}} \mathbf{L H}_{4}$. 


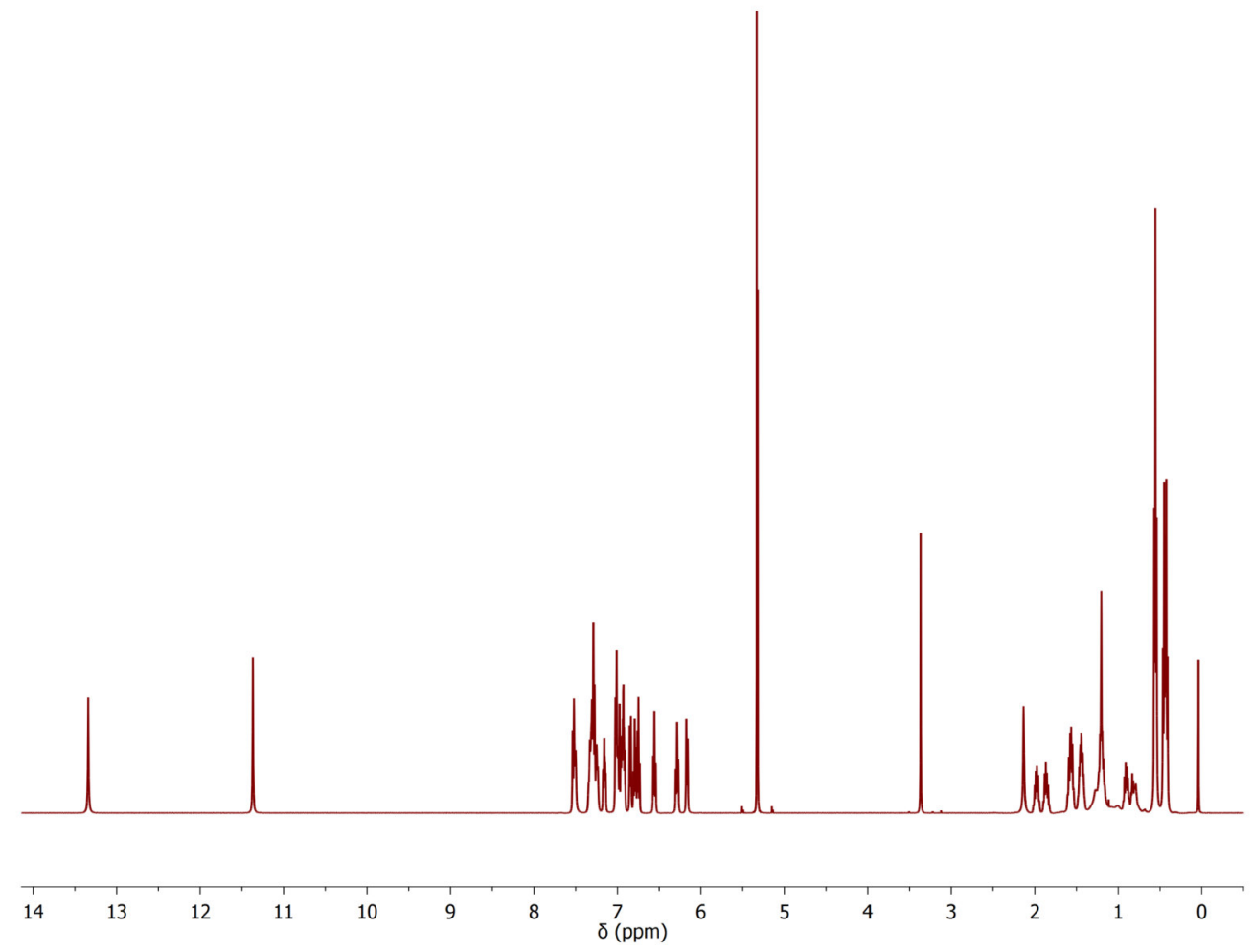

Figure A13: ${ }^{1} \mathrm{H}$ NMR spectrum $\left(500 \mathrm{MHz}, \mathrm{CD}_{2} \mathrm{Cl}_{2}, 238 \mathrm{~K}\right)$ of ${ }^{\boldsymbol{p} \mathbf{F}} \mathbf{L H}_{4}$.

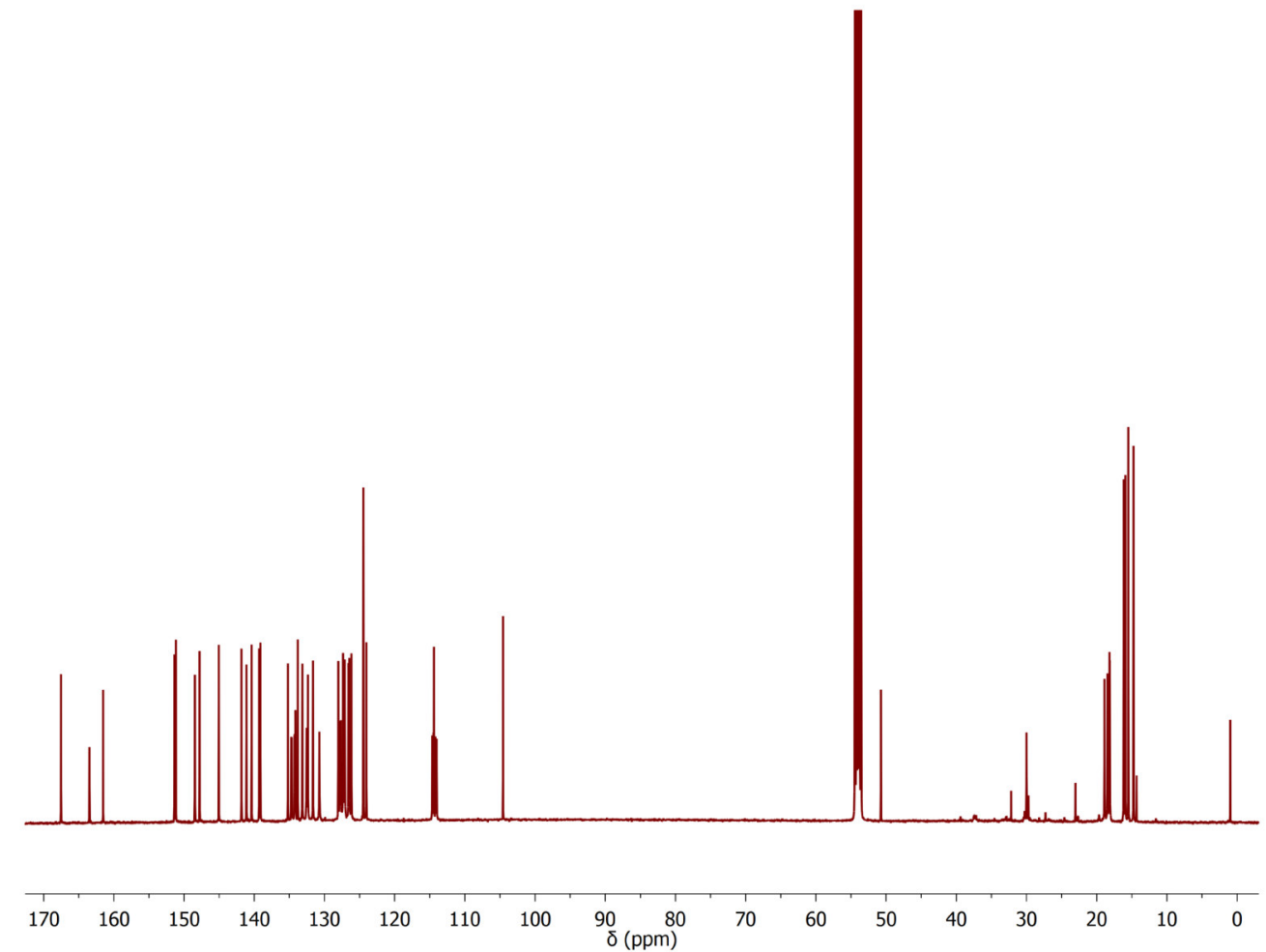

Figure A14: ${ }^{13} \mathrm{C}$ NMR spectrum $\left(125 \mathrm{MHz}, \mathrm{CD}_{2} \mathrm{Cl}_{2}, 238 \mathrm{~K}\right)$ of ${ }^{\boldsymbol{p} \mathrm{F}} \mathbf{L H}_{4}$. 


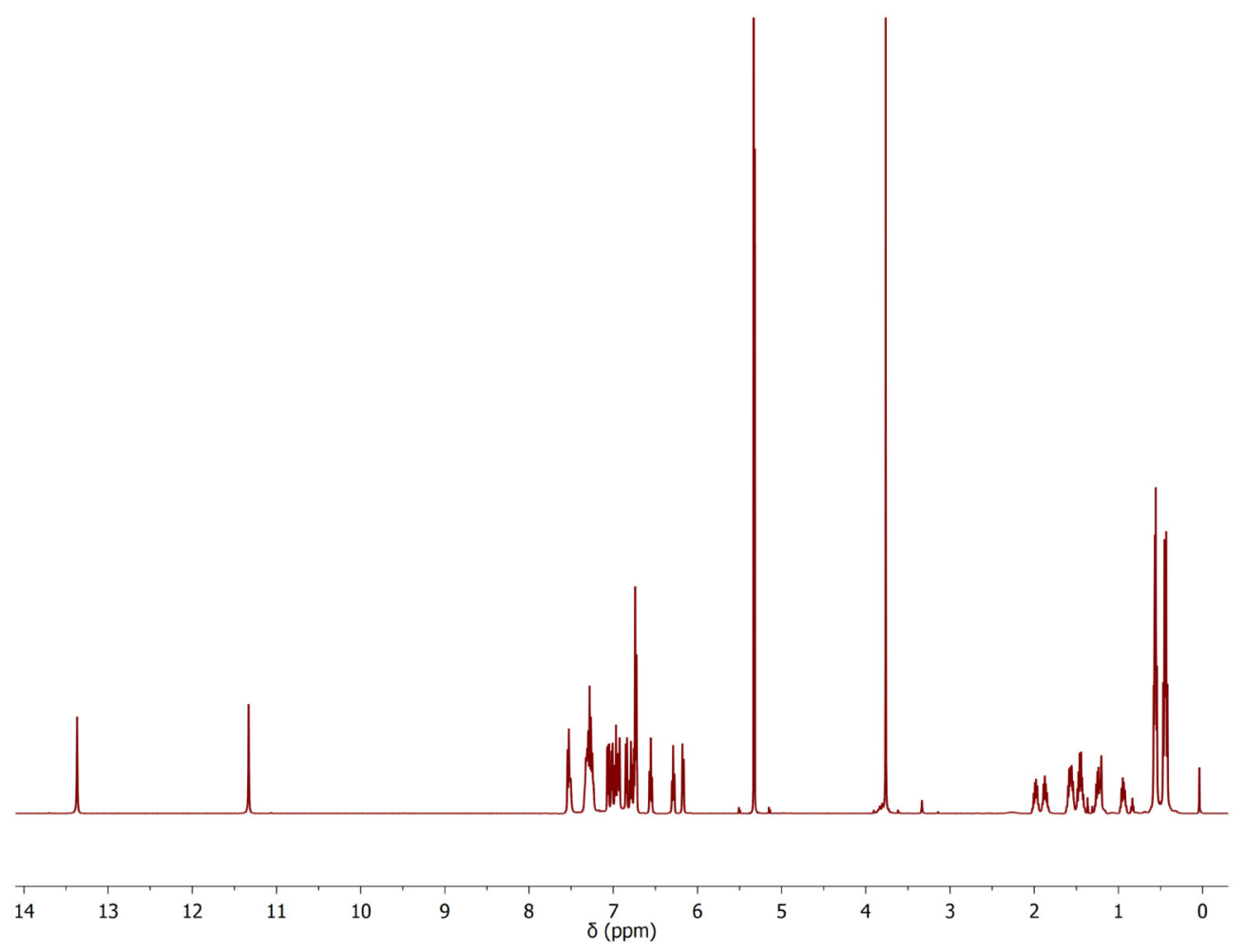

Figure A15: ${ }^{1} \mathrm{H}$ NMR spectrum $\left(500 \mathrm{MHz}, \mathrm{CD}_{2} \mathrm{Cl}_{2}, 238 \mathrm{~K}\right)$ of ${ }^{p \mathrm{MeO}} \mathbf{L H}_{4}$.

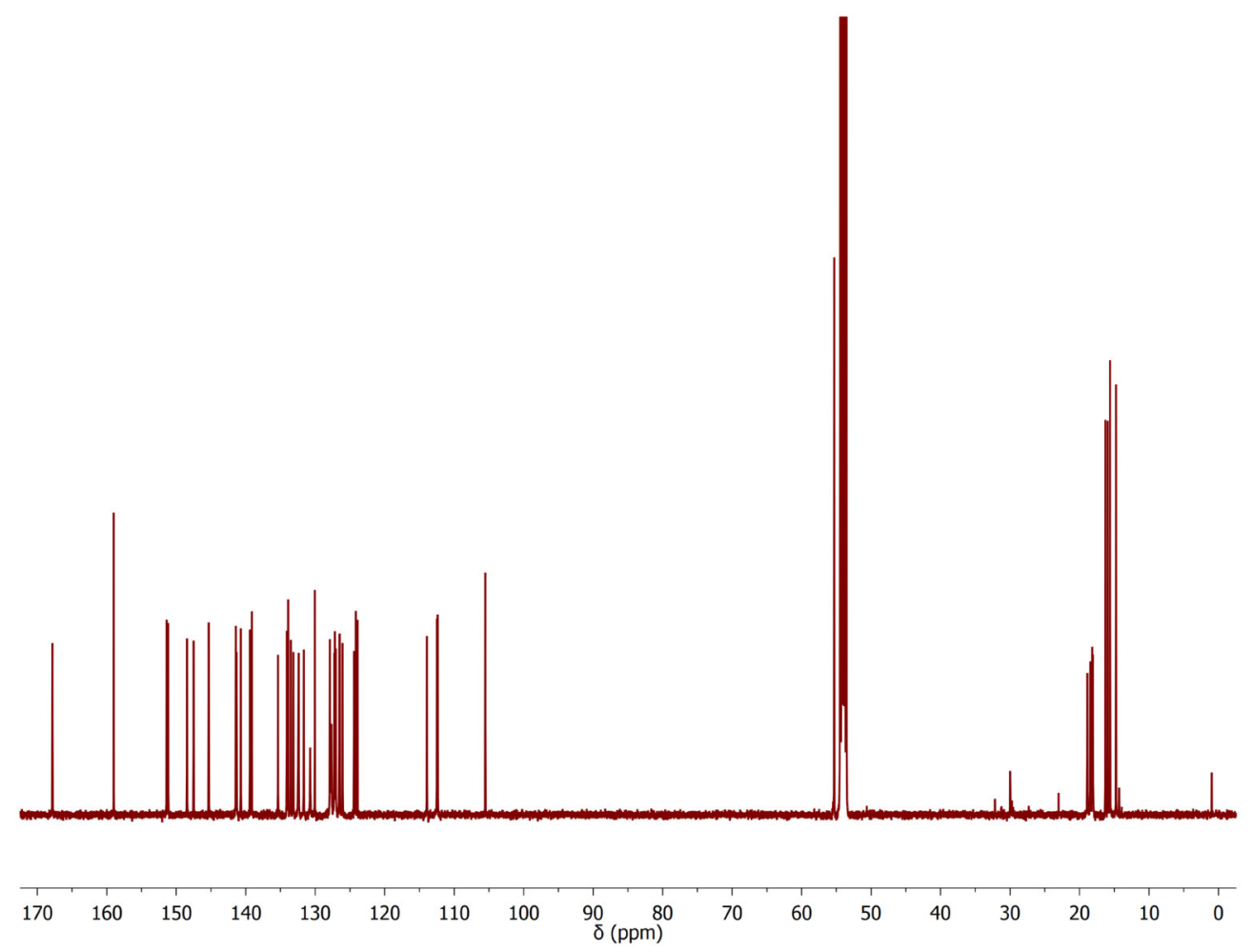

Figure A16: ${ }^{13} \mathrm{C}$ NMR spectrum $\left(125 \mathrm{MHz}, \mathrm{CD}_{2} \mathrm{Cl}_{2}, 238 \mathrm{~K}\right)$ of ${ }^{\boldsymbol{p} \mathrm{MeO}} \mathbf{L H}_{4}$. 


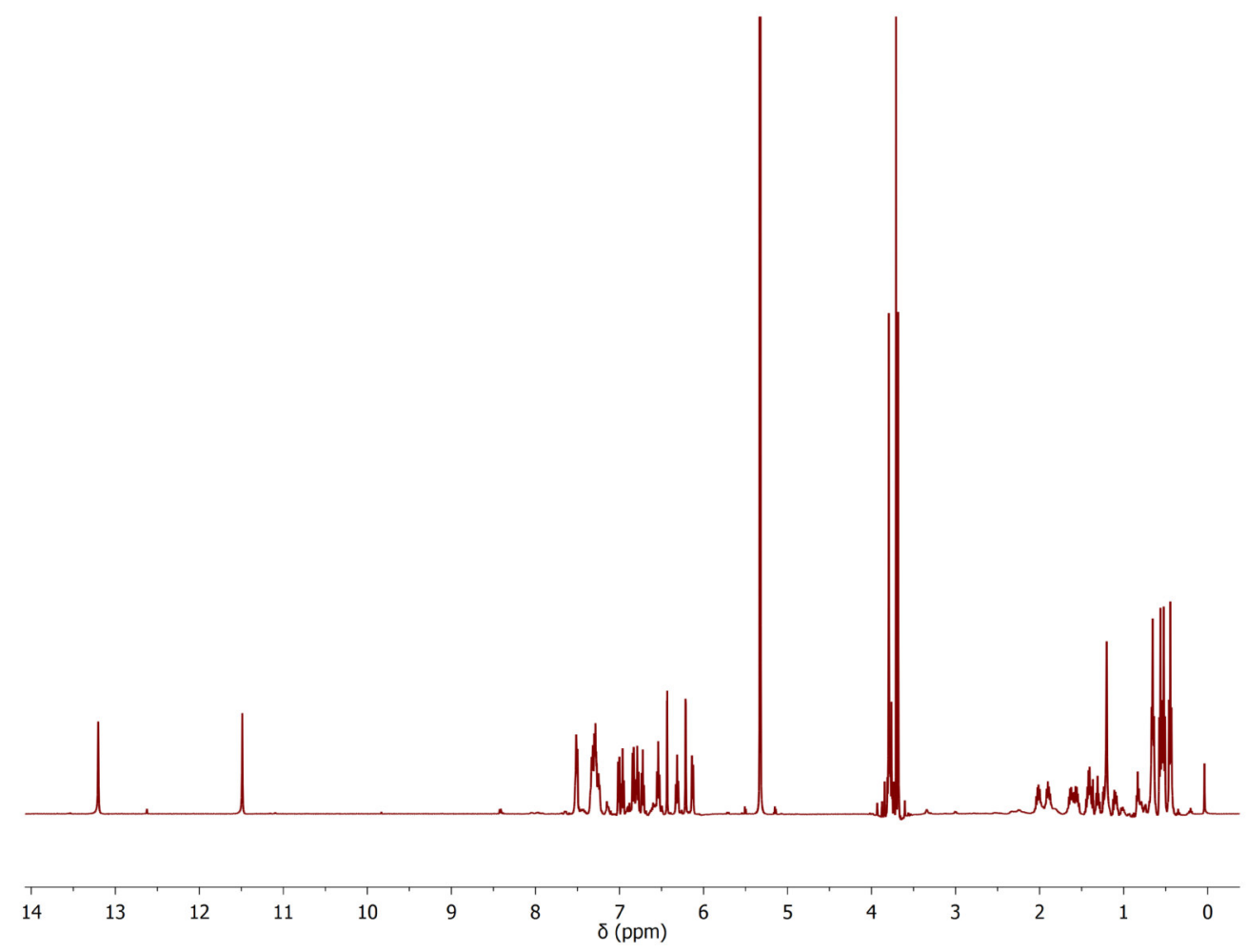

Figure A17: ${ }^{1} \mathrm{H}$ NMR spectrum $\left(500 \mathrm{MHz}, \mathrm{CD}_{2} \mathrm{Cl}_{2}, 238 \mathrm{~K}\right)$ of ${ }^{\mathrm{MeO}} \mathbf{L H}_{4}$.

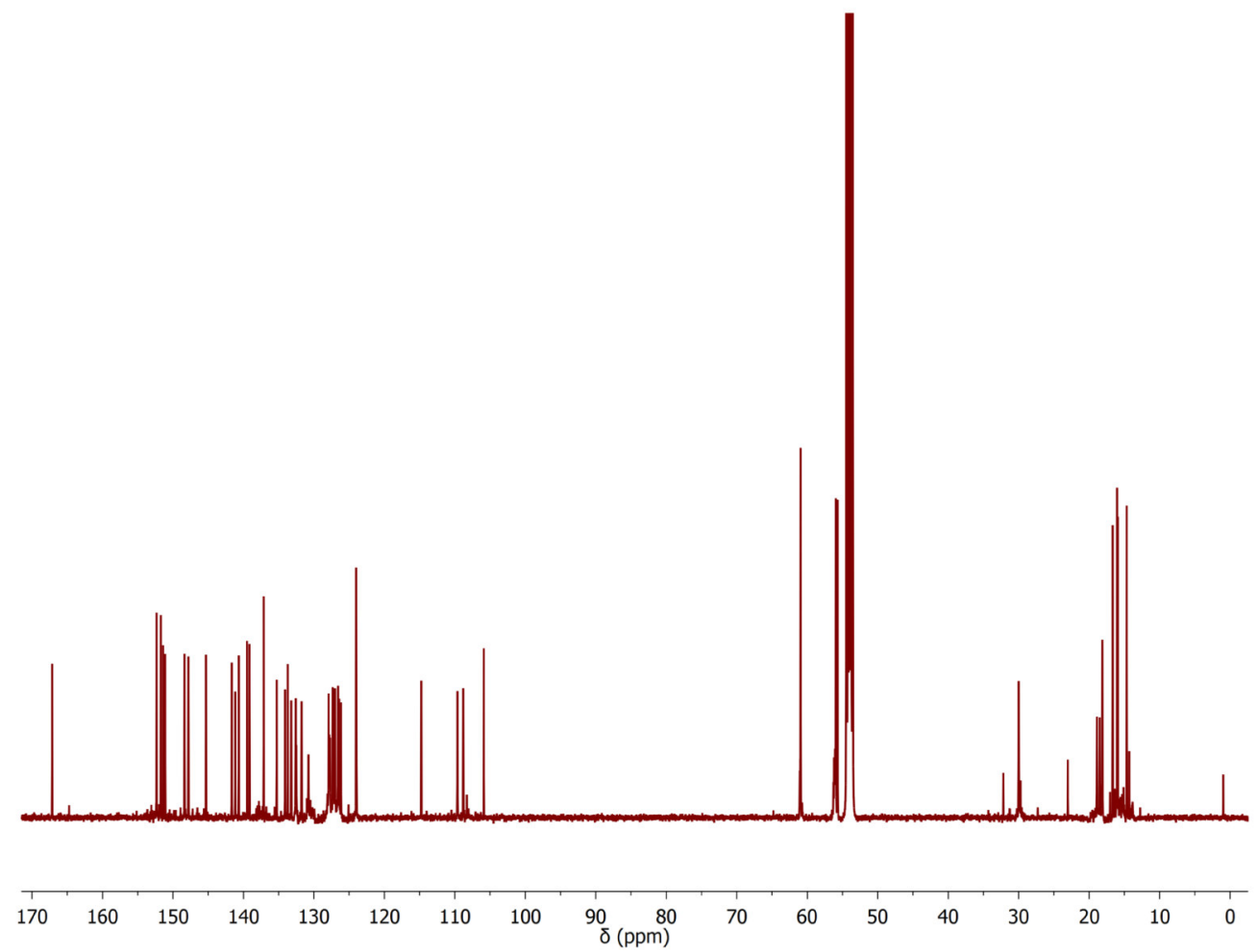

Figure A18: ${ }^{13} \mathrm{C}$ NMR spectrum $\left(125 \mathrm{MHz}, \mathrm{CD}_{2} \mathrm{Cl}_{2}, 238 \mathrm{~K}\right)$ of ${ }^{\mathrm{MeO}} \mathbf{L H}_{4}$. 


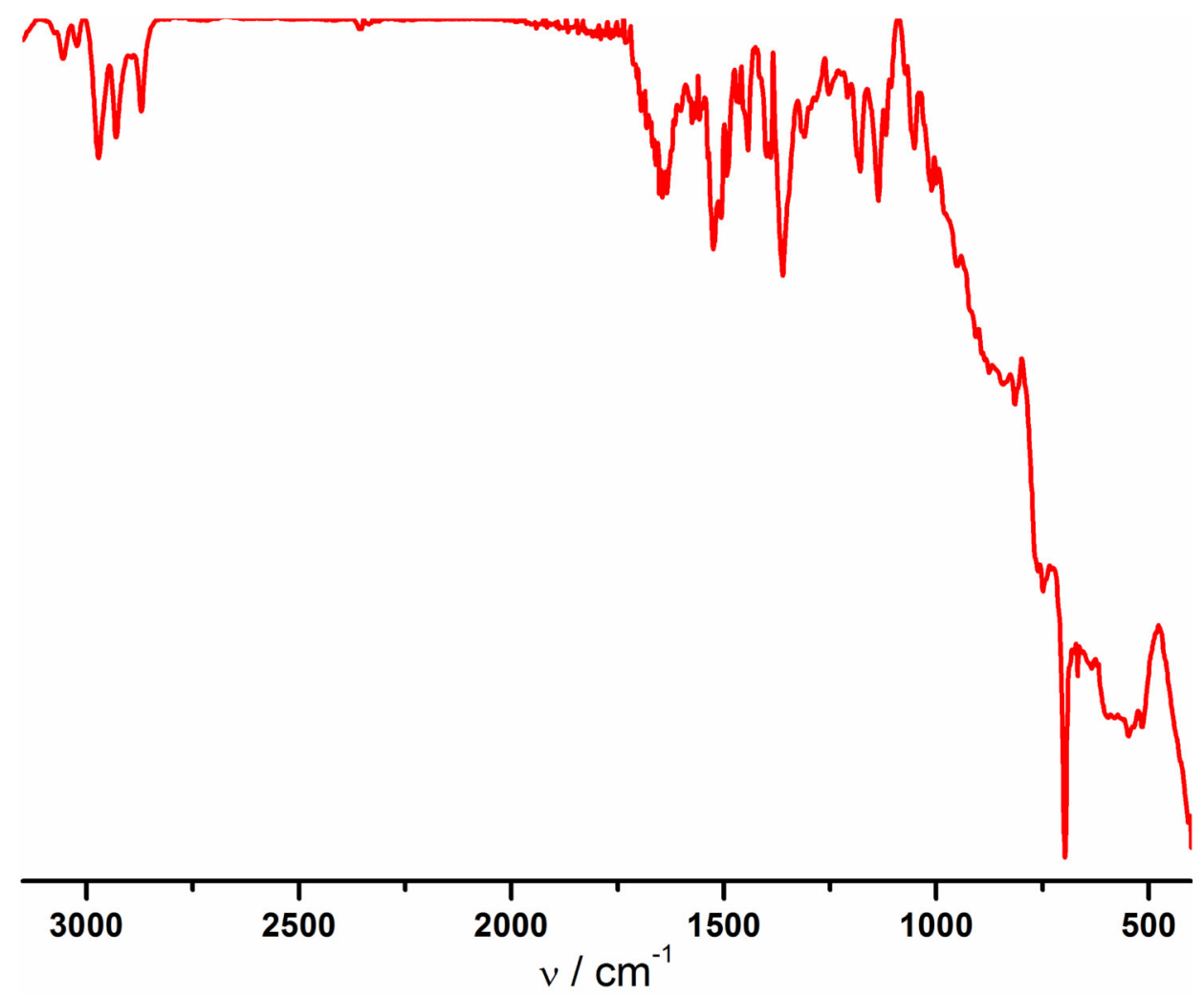

Figure A19: Infrared spectrum of ${ }^{p \mathrm{Me}} \mathbf{L H}_{4}$ (KBr pellet).

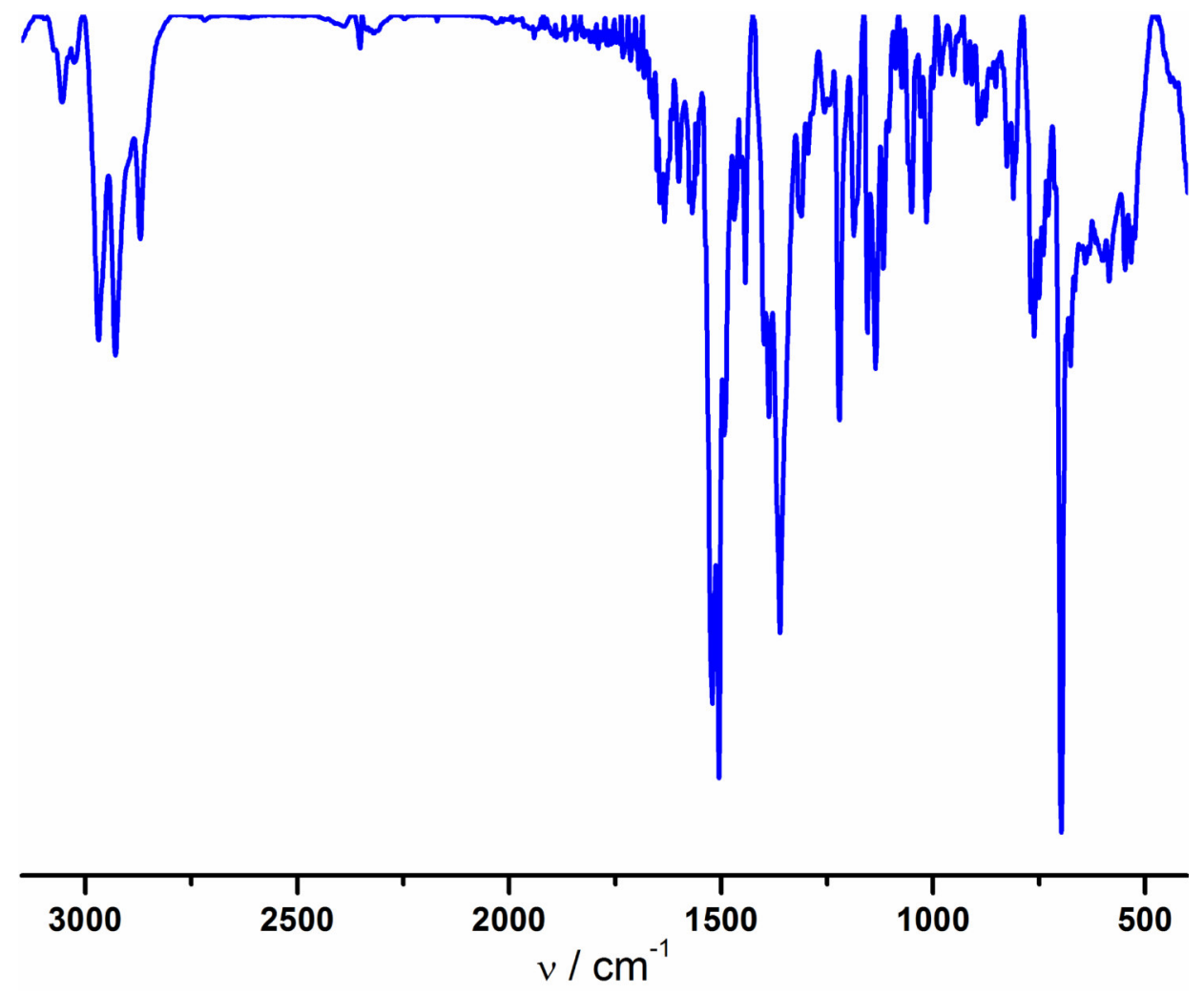

Figure A20: Infrared spectrum of ${ }^{p \mathrm{~F}} \mathbf{L H}_{4}$ (KBr pellet). 


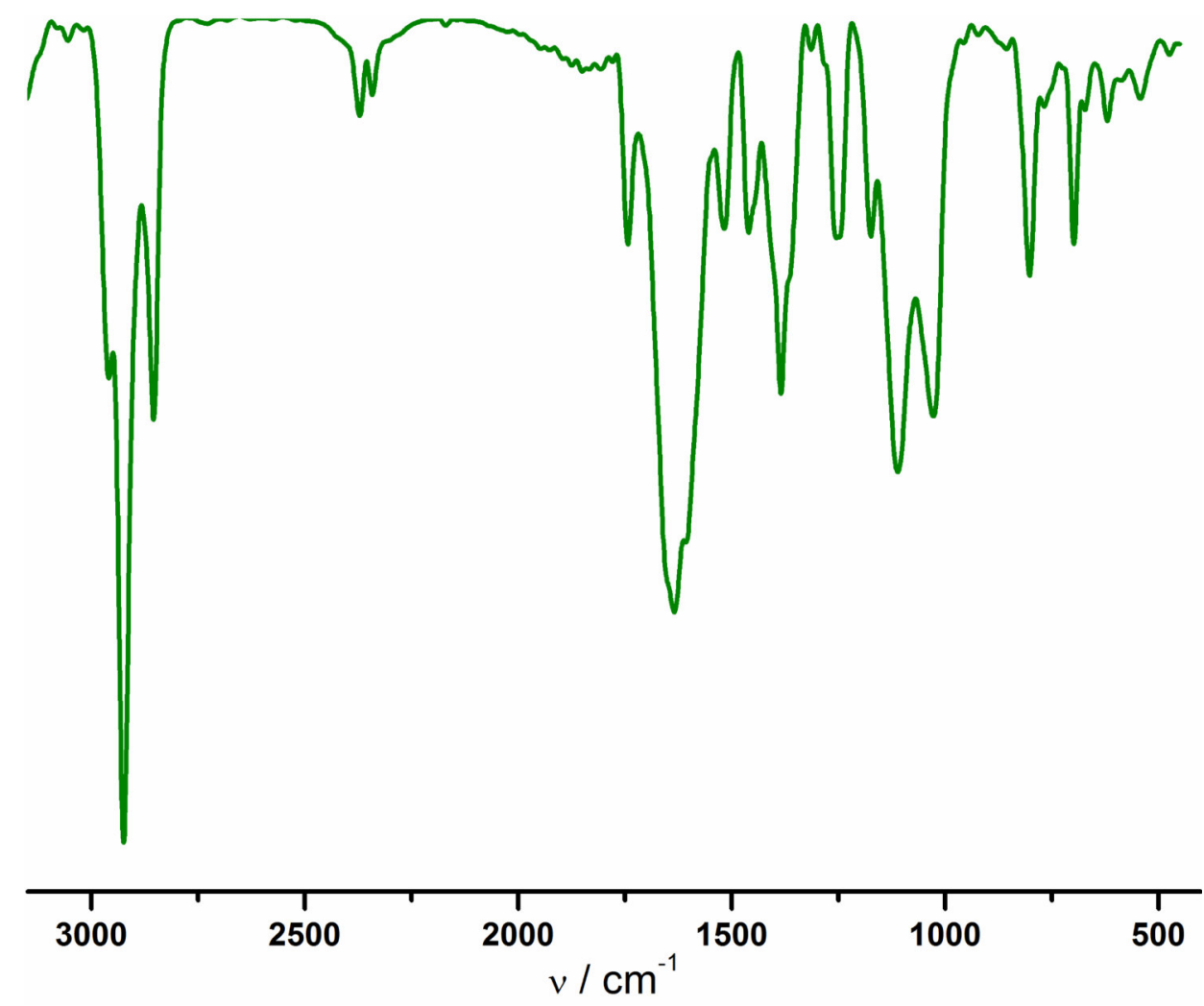

Figure A21: Infrared spectrum of ${ }^{p \mathrm{MeO}} \mathrm{LH}_{4}$ (KBr pellet).

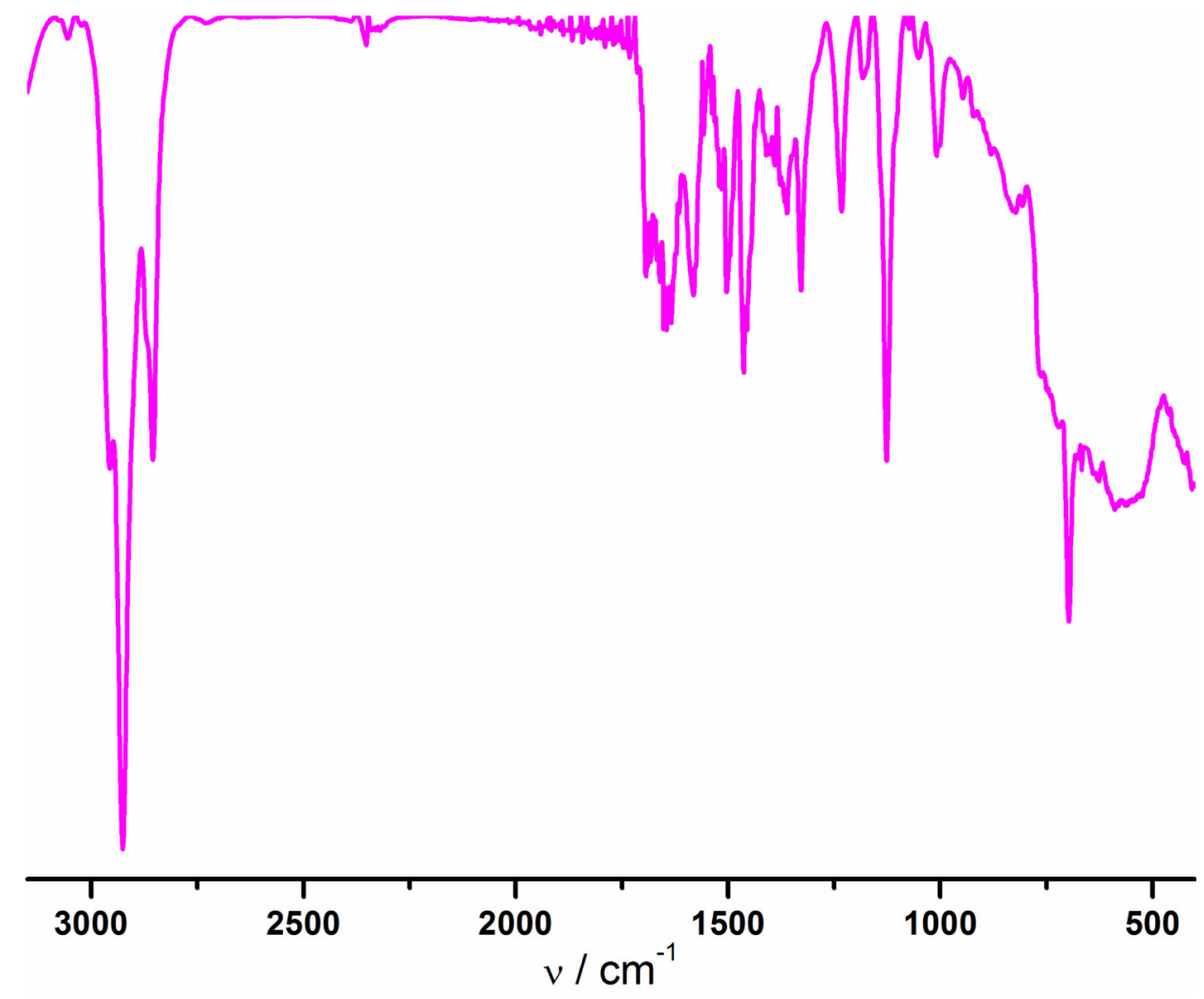

Figure A22: Infrared spectrum of ${ }^{\mathrm{MeO}} \mathrm{LH}_{4}$ (KBr pellet). 


\section{Additional Spectroscopic Data of ${ }^{\mathrm{X}} \mathrm{LCu}_{2}$}

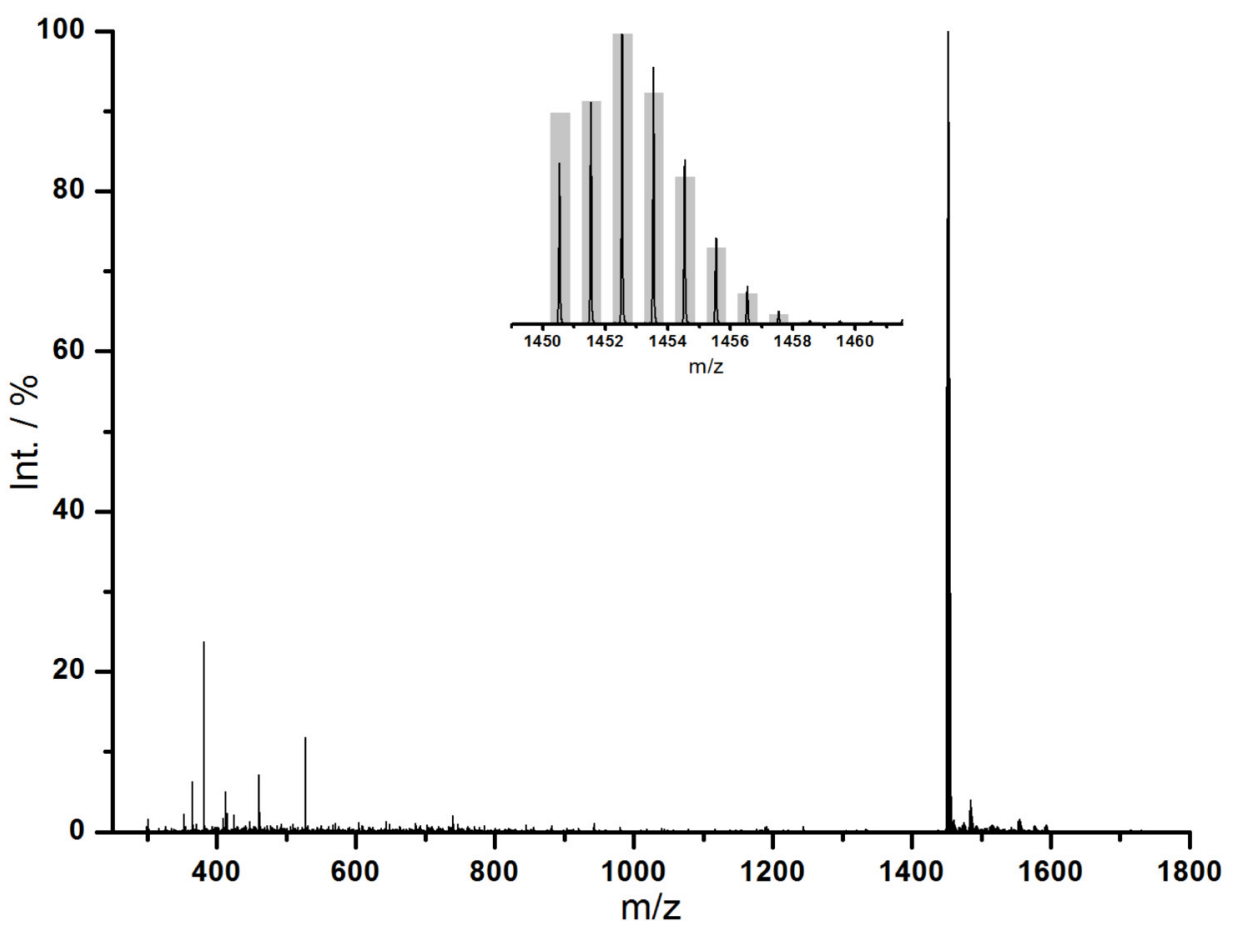

Figure A23: $\mathrm{HRMS} \mathrm{ESI}^{+}$spectrum of ${ }^{p \mathrm{Me}} \mathbf{L C u _ { 2 }}\left(\mathrm{m} / \mathrm{z}=1450.5394\right.$ (calcd. 1450.5405) $[\mathrm{M}]^{+}$) in $\mathrm{MeOH}$. Inset: Comparison of the measured (black) and calculated isotopic pattern (grey bars).

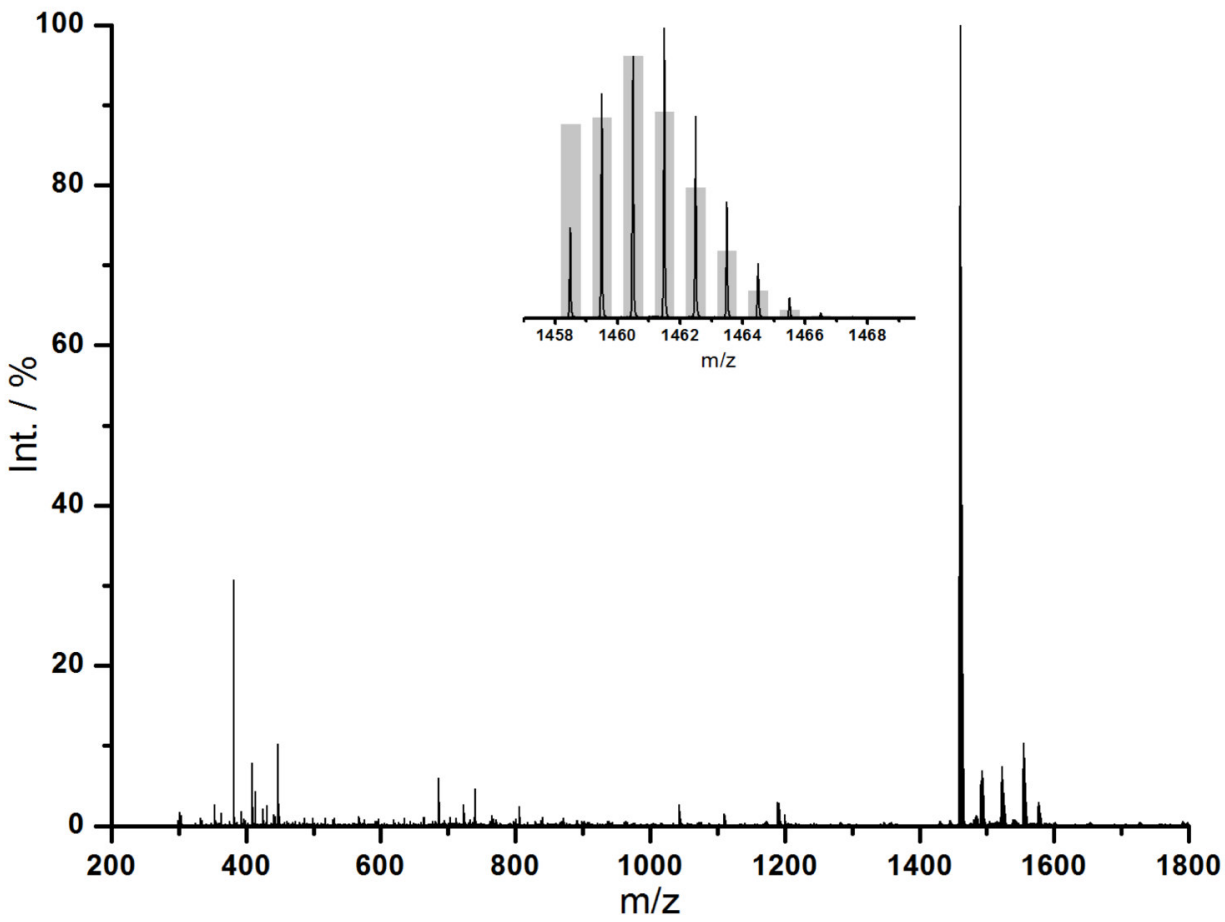

Figure A24: HRMS ESI ${ }^{+}$spectrum of ${ }^{p F}{ }^{2} \mathbf{C u}_{2}\left(\mathrm{~m} / \mathrm{z}=1458.4890\left(\right.\right.$ calcd. 1458.4904) $\left.[\mathrm{M}]^{+}\right)$in $\mathrm{MeOH}$. Inset: Comparison of the measured (black) and calculated isotopic pattern (grey bars). 


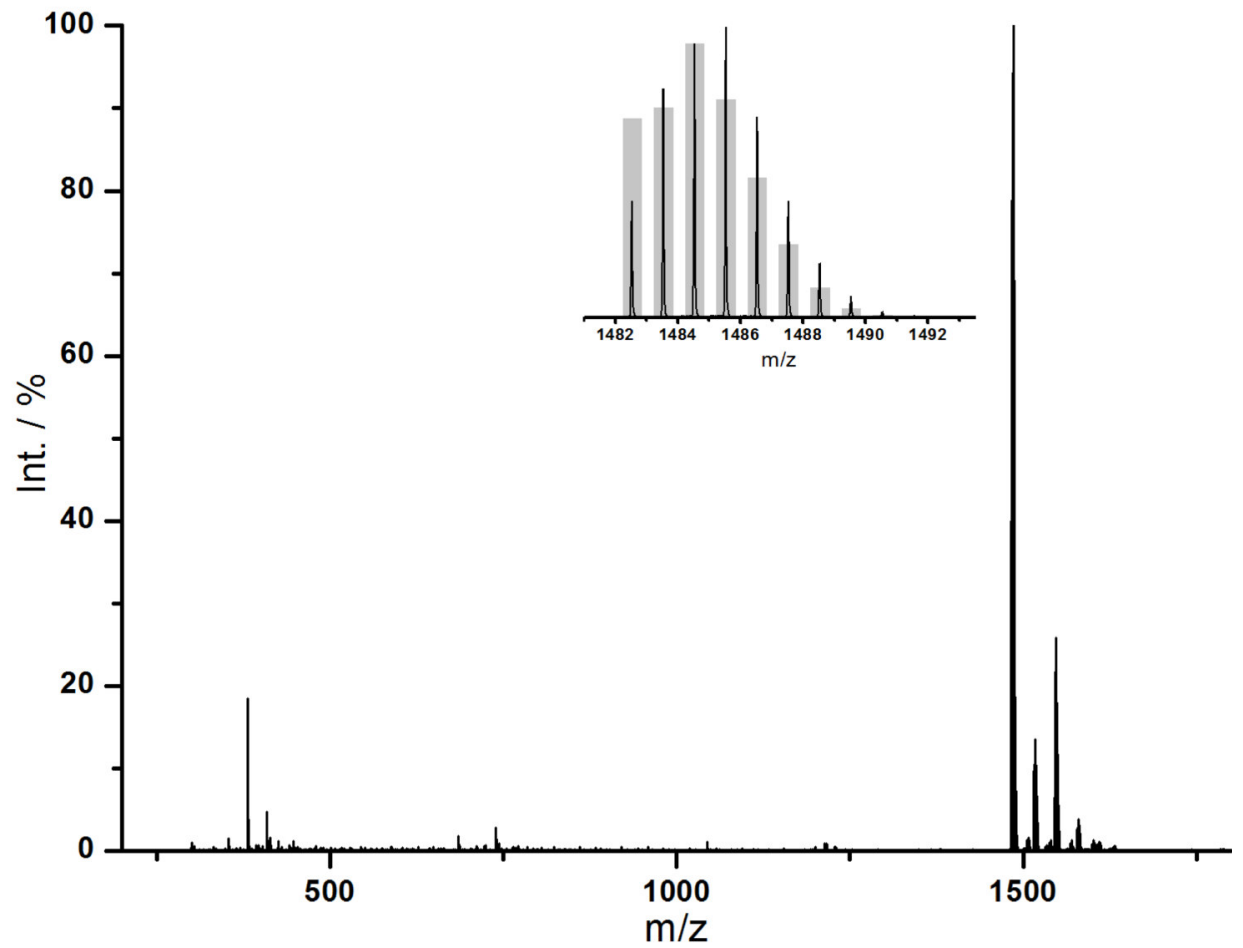

Figure A25: $\mathrm{HRMS} \mathrm{ESI}^{+}$spectrum of ${ }^{p \mathrm{MeO}} \mathbf{L C u}_{2}$ (m/z $=1482.5295$ (calcd. 1482.5304) $[\mathrm{M}]^{+}$) in $\mathrm{MeOH}$. Inset: Comparison of the measured (black) and calculated isotopic pattern (grey bars).

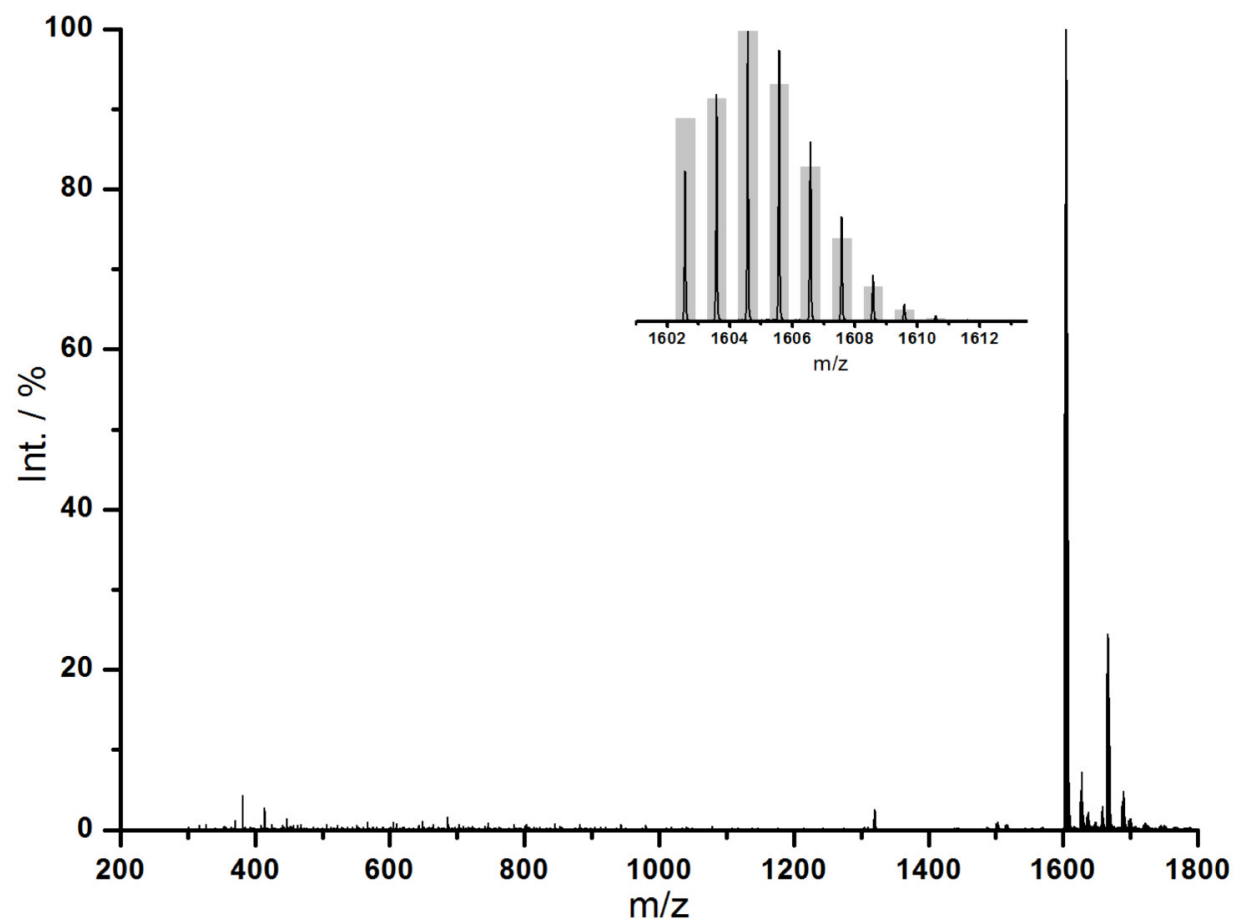

Figure A26: $\mathrm{HRMS} \mathrm{ESI}^{+}$spectrum of ${ }^{\mathrm{MeO}}{ }^{\mathbf{L C u}} \mathbf{C u}_{\mathbf{2}}\left(\mathrm{m} / \mathrm{z}=1602.5720\right.$ (calcd. 1602.5726) $[\mathrm{M}]^{+}$) in $\mathrm{MeOH}$. Inset: Comparison of the measured (black) and calculated isotopic pattern (grey bars). 


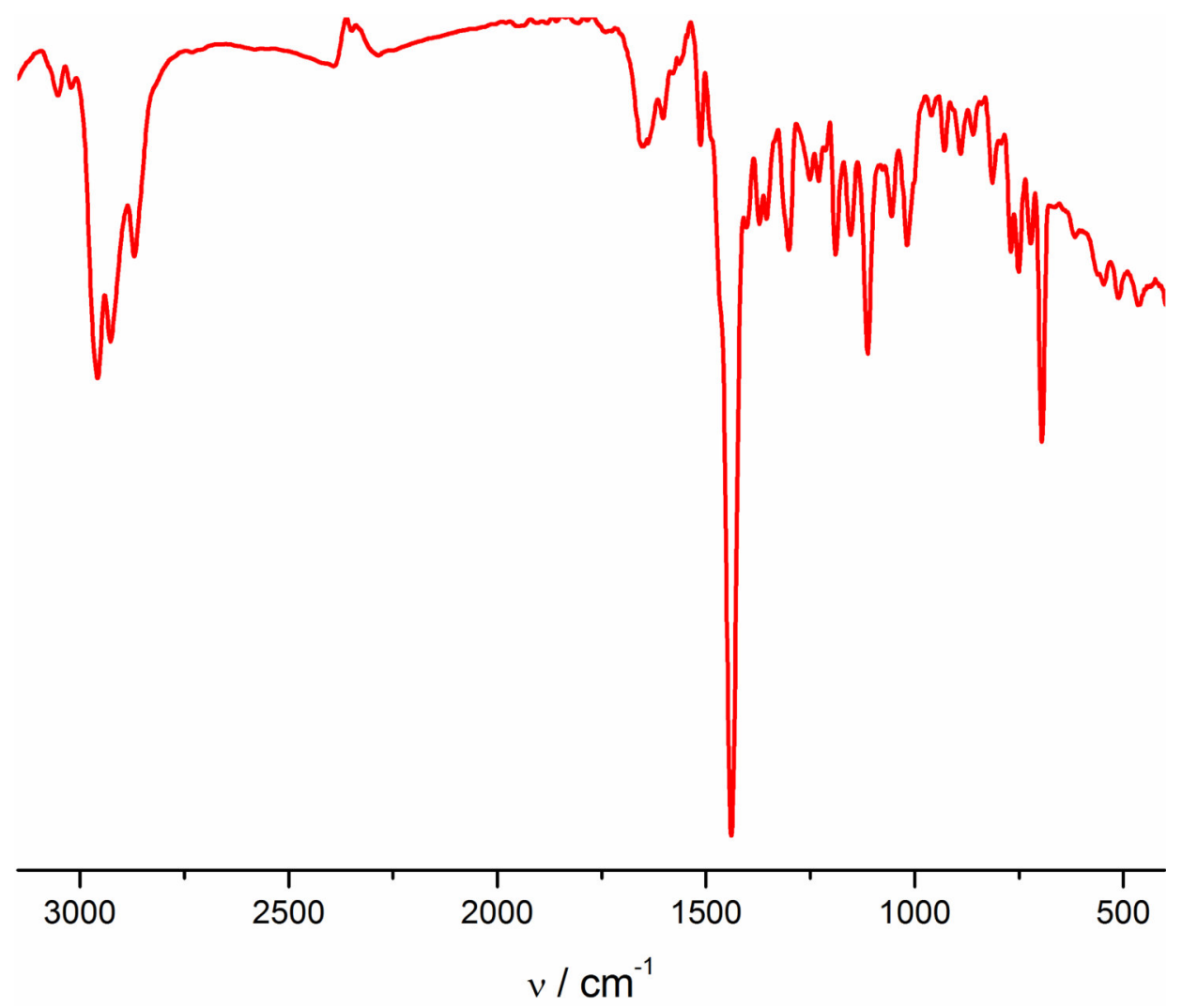

Figure A27: Infrared spectrum of ${ }^{\boldsymbol{p} \mathrm{Me}} \mathbf{L C} \mathbf{u}_{2}$ (KBr pellet).

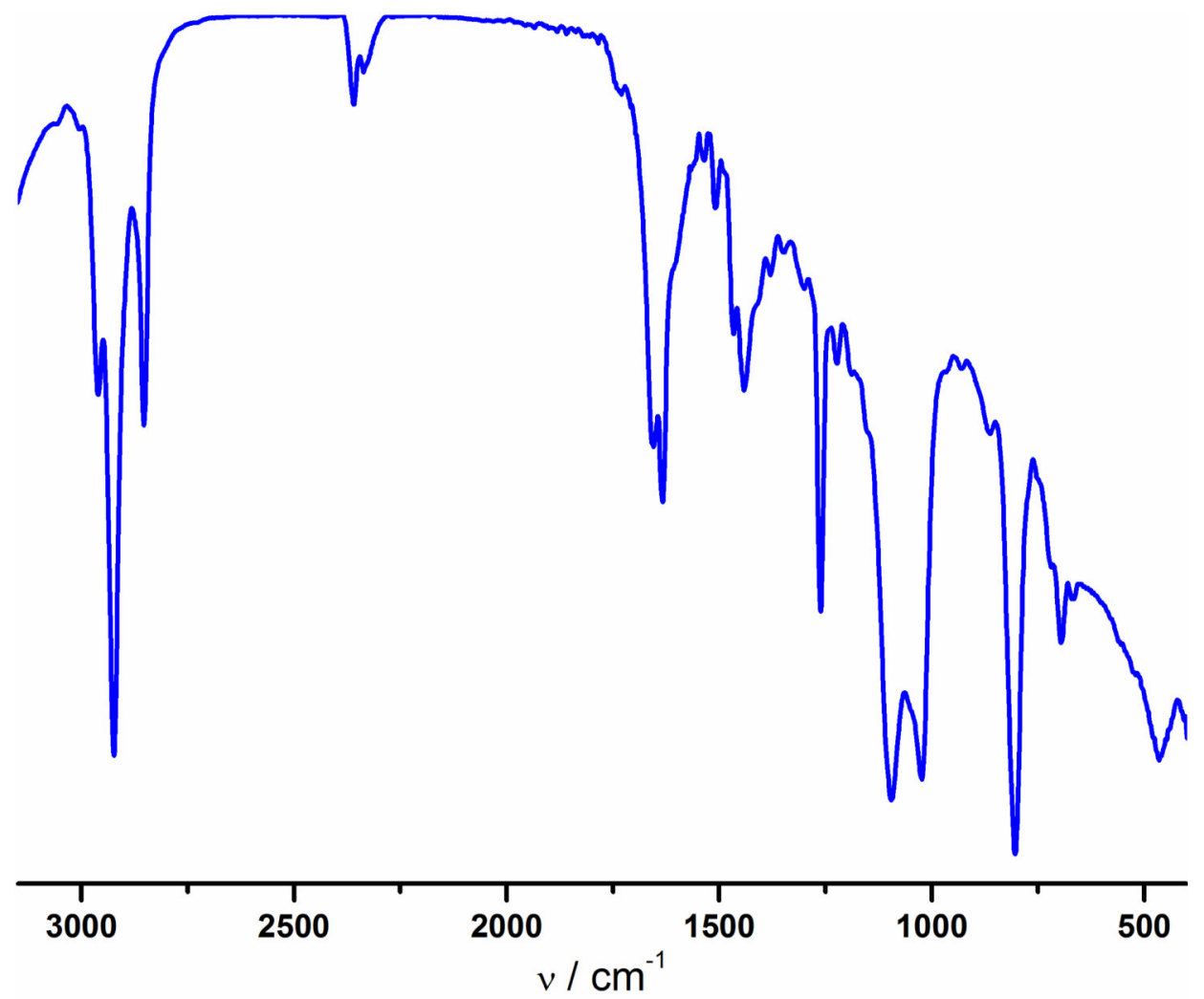

Figure A28: Infrared spectrum of ${ }^{p \mathrm{~F}} \mathbf{L C u}_{2}$ (KBr pellet). 


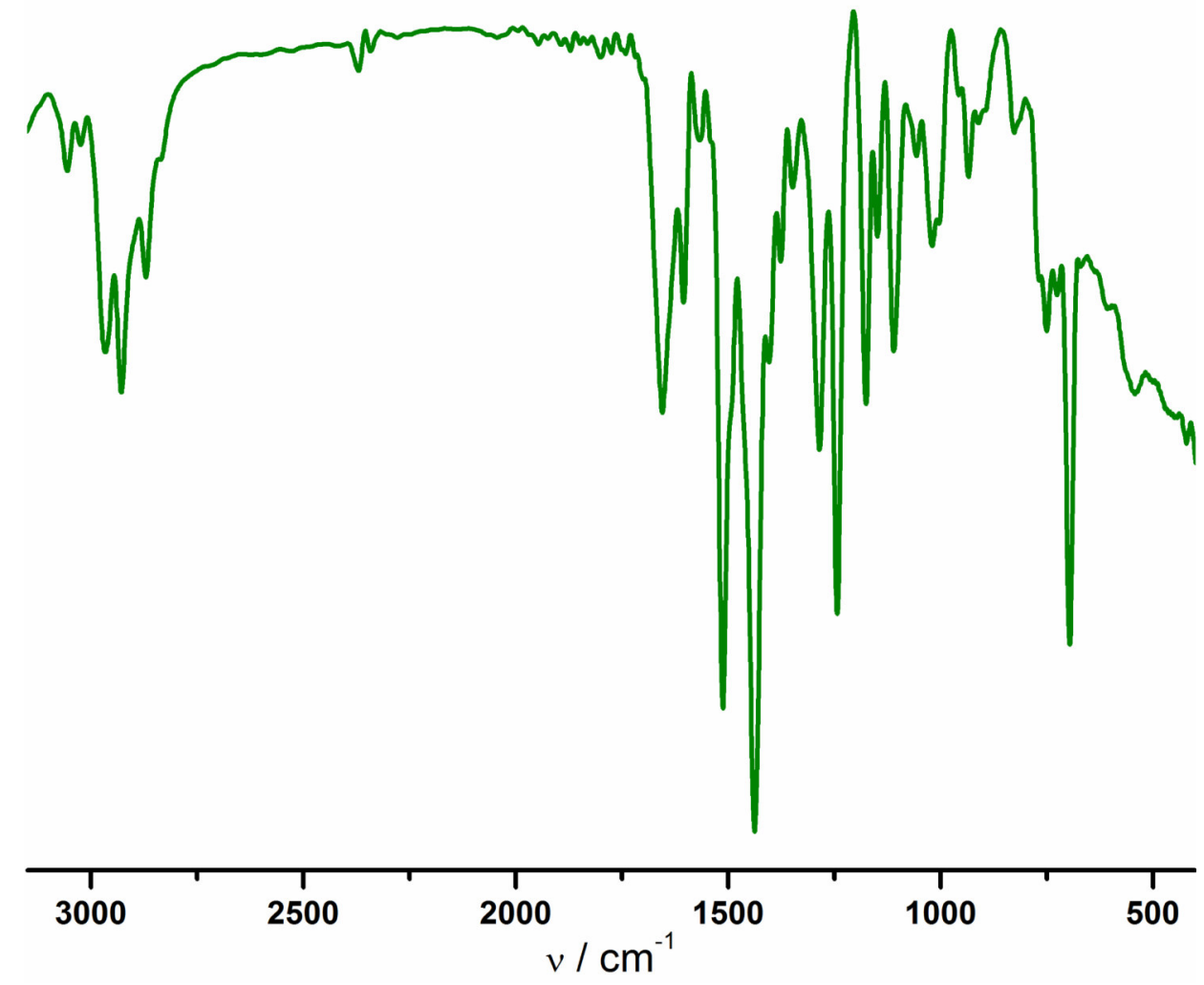

Figure A29: Infrared spectrum of ${ }^{p \mathrm{MeO}} \mathrm{LCu}_{2}$ (KBr pellet).

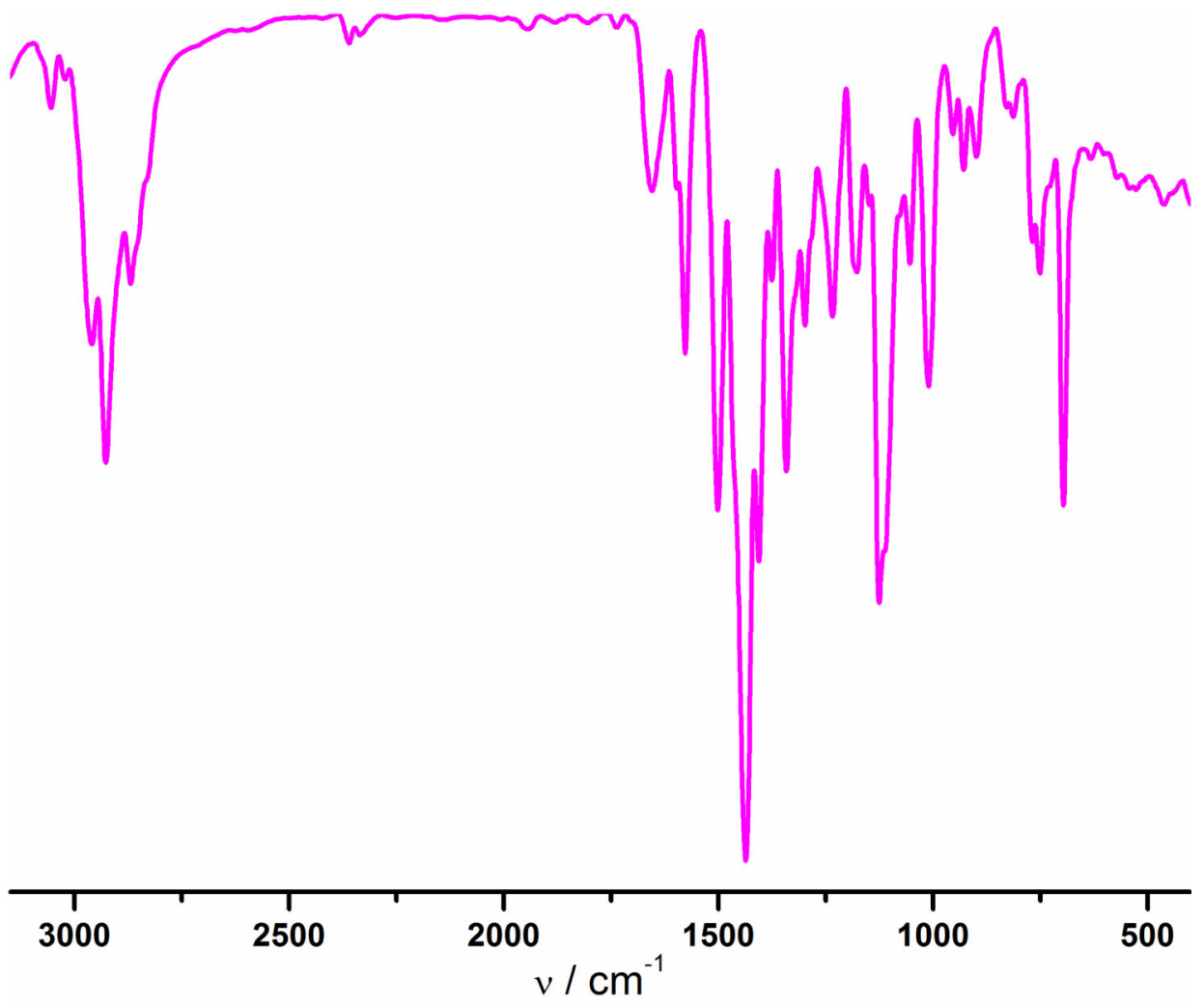

Figure A30: Infrared spectrum of ${ }^{\mathrm{MeO}} \mathbf{L C u}_{2}$ (KBr pellet). 


\section{Additional Spectroscopic Data of $\mathrm{LNiFeCl}$}

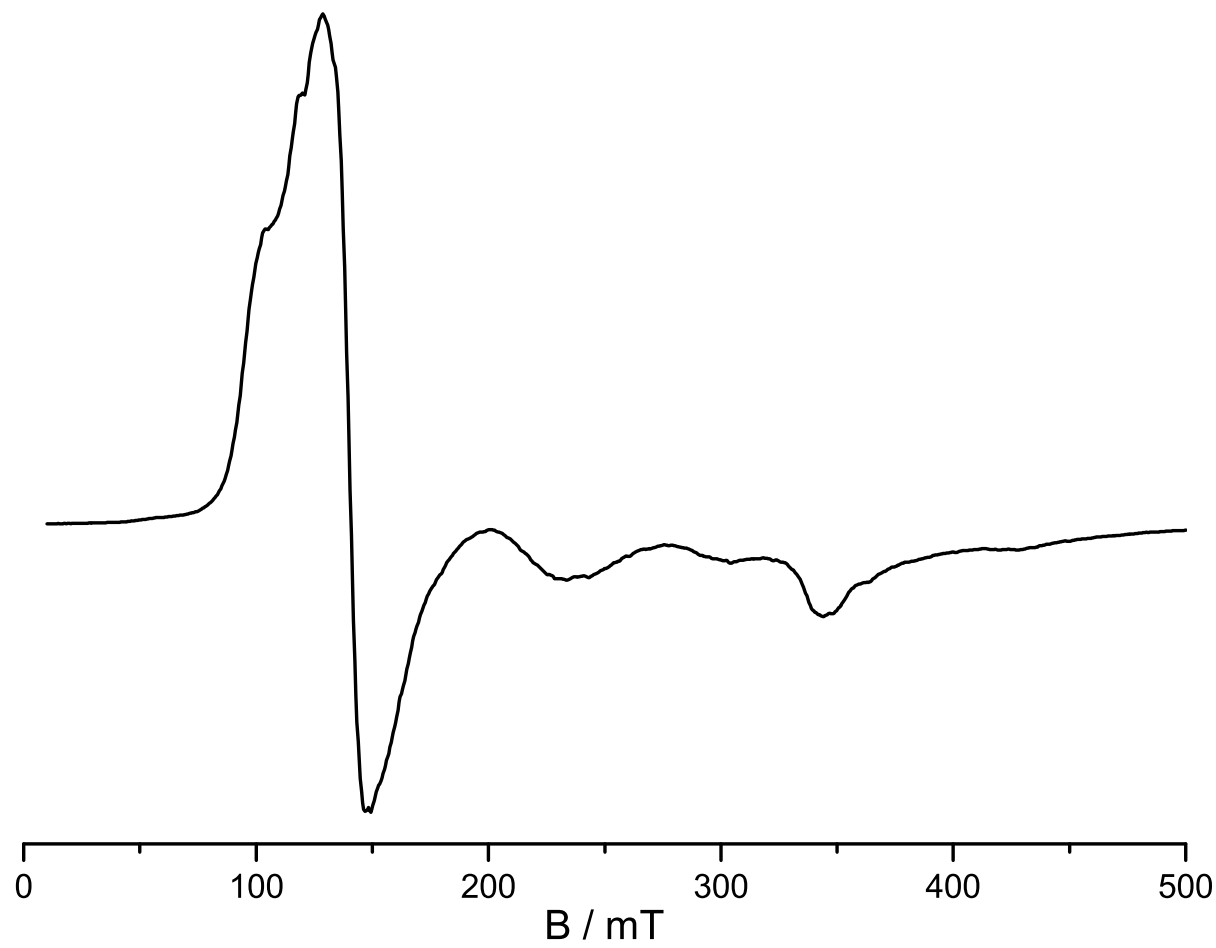

Figure A31: EPR spectrum of $\mathbf{L N i F e C l}$ in solid state at $10 \mathrm{~K}$.

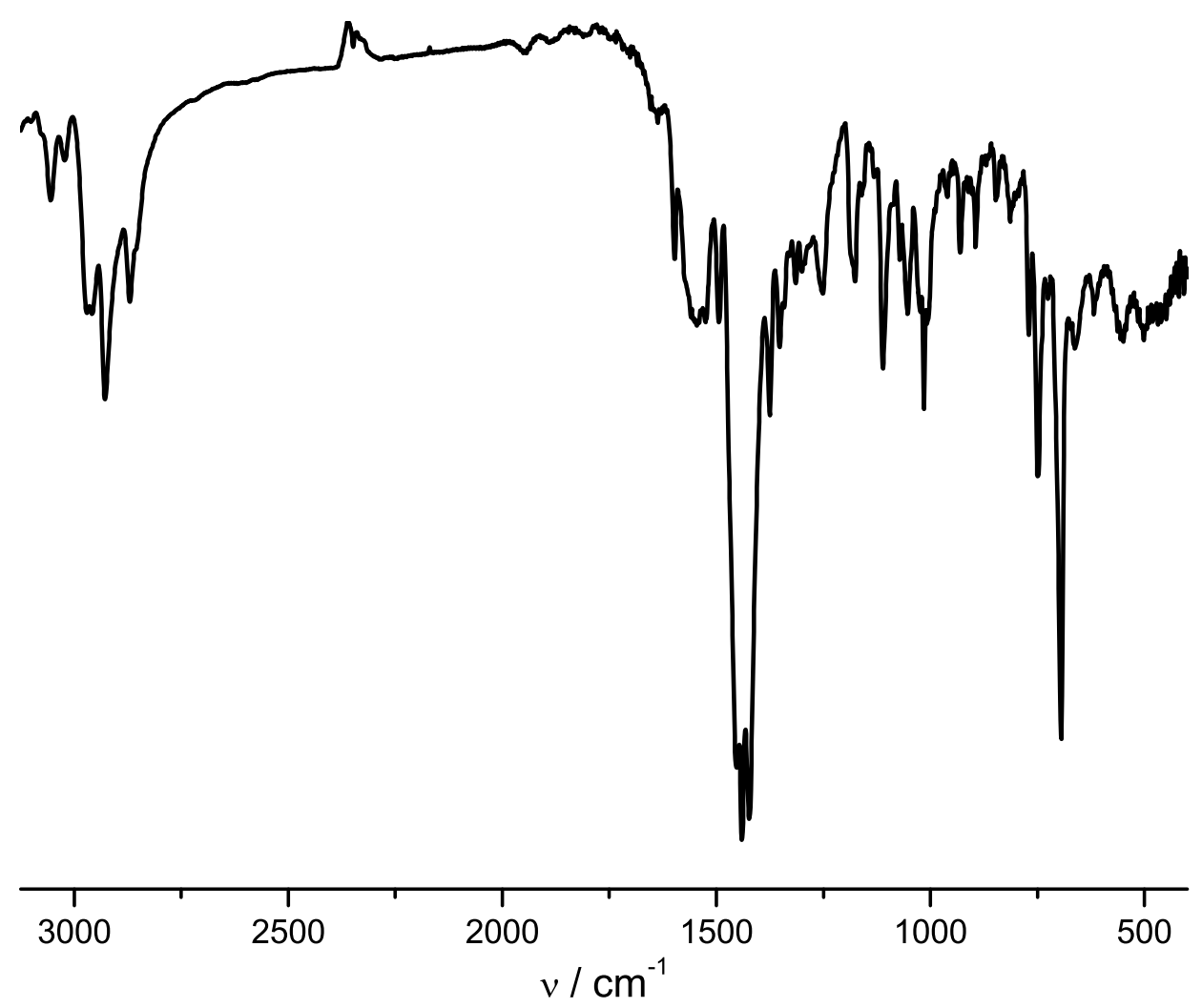

Figure A32: Infrared spectrum of LNiFeCl (KBr pellet). 


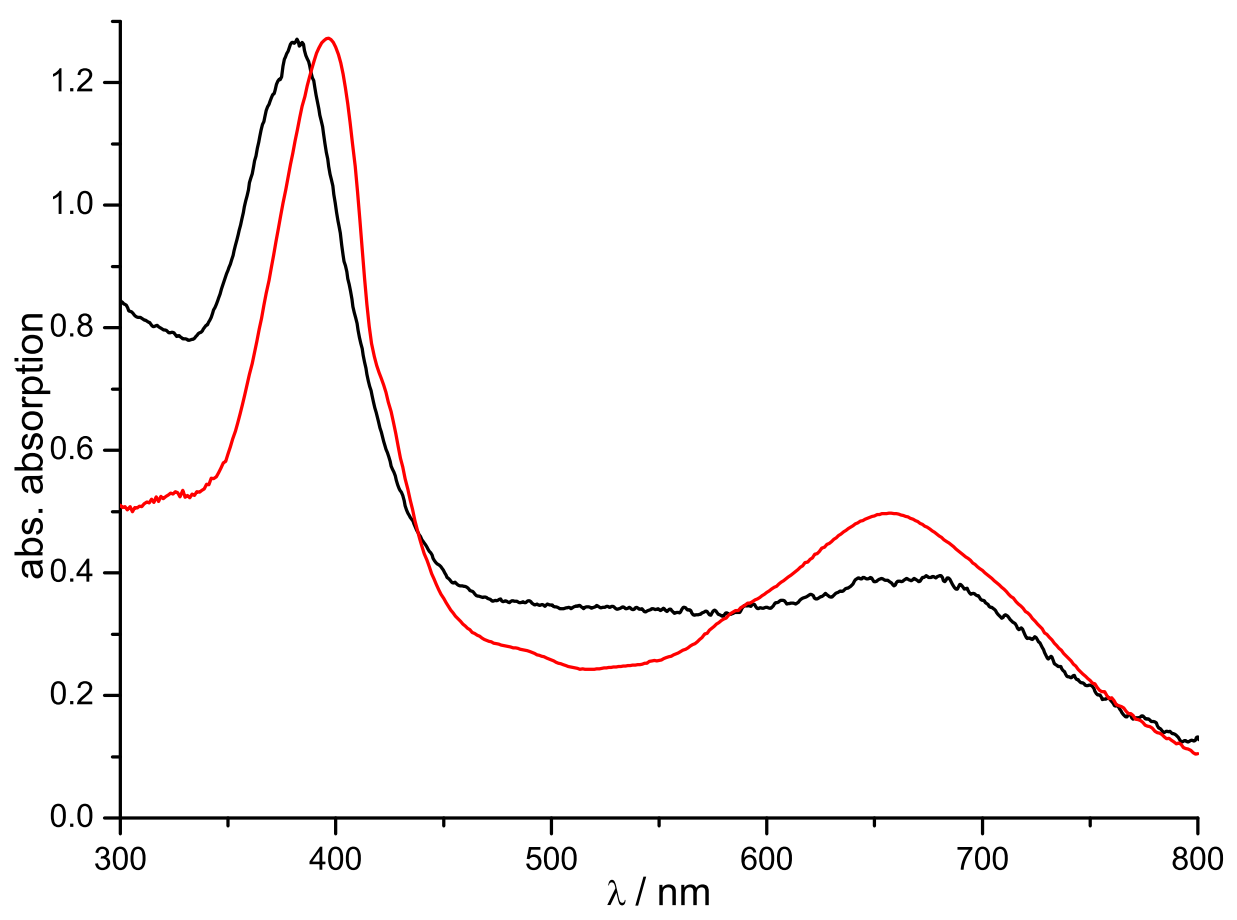

Figure A33: UV-vis spectra of LNiFe after electrochemical reduction of $\mathbf{L N i F e C l}$ in $\mathrm{CH}_{2} \mathrm{Cl}_{2}$ (black) and reduction of $\mathbf{L N i F e C l}$ with $\mathrm{CoCp}_{2}$ in THF (red). The intensity and wavelength differences result from measurements in two different solvents and the high noise to signal ratio of the electrochemical reduction. 


\section{Additional Spectroscopic Data of $\mathrm{LCuFeCl}$ and $\mathrm{L}\{\mathrm{FeCl}\}_{2}$}

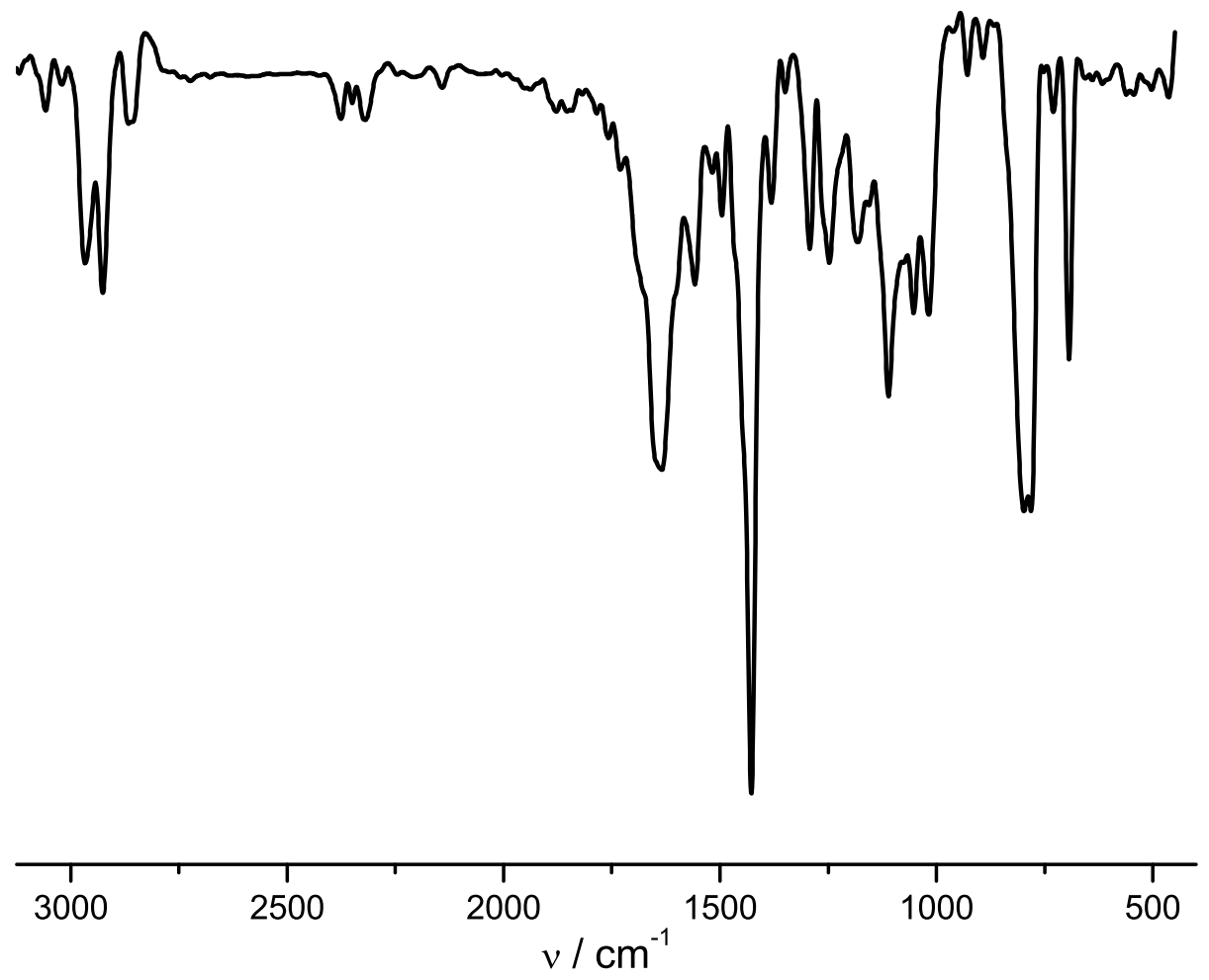

Figure A34: Infrared spectrum of $\mathbf{L C u F e C l}$ (KBr pellet).

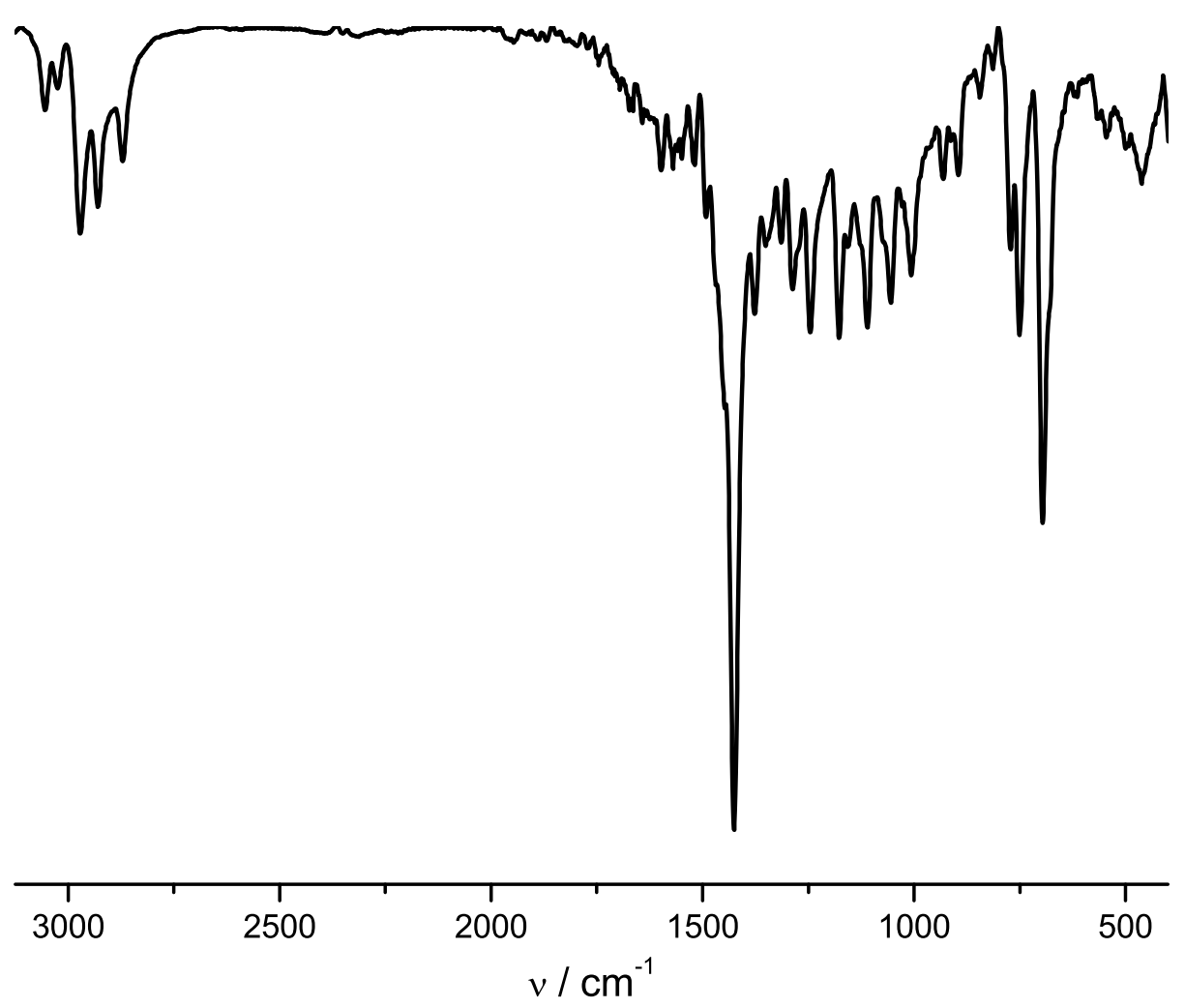

Figure A35: Infrared spectrum of $\mathbf{L}\{\mathbf{F e C l}\}_{2}$ ( $\mathrm{KBr}$ pellet). 



\section{Abbreviations}

Amb-15

Ar

$\mathrm{Cp}$

Cp*

CV

Cys

Cyt

DDQ

EP

EPR

ESI

Et

EtOAc

$\mathrm{Fc} / \mathrm{Fc}^{+}$

Fig.

Glu

HRMS

hs

IR

is

ls

$m$-CPBA

$\mathrm{Me}$

$\mathrm{MeCN}$

MMO

MS

MTBE

$\mathrm{NADH}$

NMR
Amberlyst-15

aryl

cyclopentadiene

1,2,3,4,5-pentamethylcyclopentadiene

Cyclic Voltammetry

cysteine

cytochrome

2,3-Dichloro-5,6-dicyano-1,4- $p$-benzoquinone

expanded porphyrin

Electron Paramagnetic Resonance

Electrospray Ionization

ethyl

ethylacetate

ferrocene/ferrocenium

figure

glutamate

High Resolution Mass Spectrometry

high spin

infrared

intermediate spin

low spin

meta-chloroperoxybenzoic acid

methyl

acetonitrile

methane monooxygenase

mass spectrometry

methyl tert-butyl ether

nicotinamide adenine dinucleotide

Nuclear Magnetic Resonance 
OAc

$\mathrm{Ph}$

ppm

py

pyr

pz

$\mathrm{R}$

sMMO

SQUID

STP

SWV

TFA

THF

Ts

TLC

UV-vis acetate

phenyl

parts per million

pyridine

pyrrole

pyrazole

residue

soluble methane monooxygenase

Superconducting Quantum Interference Device

Siamese-twin porphyrin

Square Wave Voltammetry

trifluoroacetic acid

tetrahydrofuran

tosyl

Thin Layer Chromatography

ultraviolet-visible 


\section{Bibliography}

[1] D. Dolphin, editor, The porphyrins, Acad. Press, New York, 1978-1979.

[2] Ivano Bertini, Harry B. Gray, Edward I. Stiefel, Joan Selverstone Valentine, Biological Inorganic Chemistry - Structure $\&$ Reactivity, University Science Books, New Jersey, 2007.

[3] W. Kaim, B. Schwederski, Bioanorganische Chemie, Teubner Studienbücher Chemie, 4th edition, Teubner, Wiesbaden, 2010.

[4] O. Q. Munro, J. C. Bradley, R. D. Hancock, H. M. Marques, F. Marsicano, P. W. Wade, J. Am. Chem. Soc., 1992, 114, 7218-7230.

[5] M. Sono, M. P. Roach, E. D. Coulter, J. H. Dawson, Chem. Rev., 1996, 96, 28412888 .

[6] J. Liu, S. Chakraborty, P. Hosseinzadeh, Y. Yu, S. Tian, I. Petrik, A. Bhagi, Y. Lu, Chem. Rev., 2014, 114, 4366-4469.

[7] P. Khirsariya, R. K. Mewada, Procedia Eng., 2013, 51, 409-415.

[8] K. M. Kadish, K. M. Smith, R. Guilard, Inorganic, organometallic and coordination chemistry, volume 3 of The porphyrin handbook, Academic Press, San Diego, Calif., 2000 .

[9] F. A. Walker, U. Simonis, Iron Porphyrin Chemistry, in R. A. Scott, editor, Encyclopedia of inorganic and bioinorganic chemistry, Wiley, Chichester, 2012.

[10] L. K. Frensch, K. Pröpper, M. John, S. Demeshko, C. Brückner, F. Meyer, Angew. Chem. Int. Ed., 2011, 50, 1420-1424.

[11] T. EIcher, S. Hauptmann, The Chemistry of Heterocycles, Wiley-VCH, Weinheim, 2003.

[12] E. Hückel, Z. Physik, 1931, 70, 204-286.

[13] C. Rimington, Biochem. J., 1960, 75, 620-623.

[14] M. Gouterman, J. Mol. Spectrosc, 1961, 6, 138-163. 
[15] L. M. Mink, M. L. Neitzel, L. M. Bellomy, R. E. Falvo, R. K. Boggess, Polyhedron, 1997, 16, 2809-2817.

[16] K. M. Kadish, K. M. Smith, R. Guilard, editors, The porphyrin handbook, Academic Press, San Diego, Calif., 2000.

[17] J.-G. Liu, T. Ohta, S. Yamaguchi, T. Ogura, S. Sakamoto, Y. Maeda, Y. Naruta, Angew. Chem. Int. Ed., 2009, 48, 9262-9267.

[18] R. Misra, T. K. Chandrashekar, Acc. Chem. Res., 2008, 41, 265-279.

[19] M. Stępień, L. Latos-Grażyński, Top. Heterocycl. Chem., 2009, 19, 83-153.

[20] S. Saito, A. Osuka, Angew. Chem. Int. Ed., 2011, 50, 4342-4373.

[21] Y. Pareek, M. Ravikanth, T. K. Chandrashekar, Acc. Chem. Res., 2012, 45, 18011816.

[22] S. Shimizu, N. Aratani, A. Osuka, Chem. Eur. J., 2006, 12, 4909-4918.

[23] H. Rath, J. Sankar, V. Prabhuraja, T. K. Chandrashekar, A. Nag, D. Goswami, J. Am. Chem. Soc., 2005, 127, 11608-11609.

[24] M. Rajeswara Rao, M. Ravikanth, Eur. J. Org. Chem., 2011, 2011, 1335-1345.

[25] Y. Ding, W.-H. Zhu, Y. Xie, Chem. Rev., 2016.

[26] W.-Y. Cha, T. Soya, T. Tanaka, H. Mori, Y. Hong, S. Lee, K. H. Park, A. Osuka, D. Kim, Chem. Commun., 2016, 52, 6076-6078.

[27] J. L. Sessler, D. Seidel, Angew. Chem. Int. Ed., 2003, 42, 5134-5175.

[28] G. R. Peterson, N. Bampos, Angew. Chem. Int. Ed., 2010, 49, 3930-3933.

[29] J. Skonieczny, L. Latos-Grażyński, L. Szterenberg, Org. Biomol. Chem., 2012, 10, 3463-3471.

[30] Y. Chang, H. Chen, Z. Zhou, Y. Zhang, C. Schutt, R. Herges, Z. Shen, Angew. Chem. Int. Ed., 2012, 51, 12801-12805.

[31] M. Gosmann, B. Franck, Angew. Chem. Int. Ed. Engl., 1986, 25, 1100-1101.

[32] K. M. Kadish, K. M. Smith, R. Guilard, Synthesis and organic chemistry, volume 1 of The porphyrin handbook, Academic Press, San Diego, Calif., 2000.

[33] T. Soya, W. Kim, D. Kim, A. Osuka, Chem. Eur. J., 2015, 21, 8341-8346.

[34] S. Mori, A. Osuka, J. Am. Chem. Soc., 2005, 127, 8030-8031.

[35] S. Mori, S. Shimizu, J.-Y. Shin, A. Osuka, Inorg. Chem., 2007, 46, 4374-4376. 
[36] S. Mori, A. Osuka, Inorg. Chem., 2008, 47, 3937-3939.

[37] J. L. Sessler, E. Tomat, Acc. Chem. Res., 2007, 40, 371-379.

[38] J. L. Sessler, R. A. Miller, Biochem. Pharmacol., 2000, 59, 733-739.

[39] S. W. Young, F. Qing, A. Harriman, J. L. Sessler, W. C. Dow, T. D. Mody, G. W. Hemmi, Y. Hao, R. A. Miller, PNAS, 1996, 93, 6610-6615.

[40] S. W. Young, F. Qing, A. Harriman, J. L. Sessler, W. C. Dow, T. D. Mody, G. W. Hemmi, Y. Hao, R. A. Miller, PNAS, 1999, 96, 2569.

[41] J. M. Keca, J. Chen, M. Overchuk, N. Muhanna, C. M. MacLaughlin, C. S. Jin, W. D. Foltz, J. C. Irish, G. Zheng, Angew. Chem. Int. Ed., 2016, 55, 6187-6191.

[42] J. L. Sessler, G. Hemmi, T. D. Mody, T. Murai, A. Burrell, S. W. Young, Acc. Chem. Res., 1994, 27, 43-50.

[43] K. Naoda, Y. M. Sung, J. M. Lim, D. Kim, A. Osuka, Chem. Eur. J., 2014, 20, 7698-7705.

[44] E. J. Lind, Ph.D. Thesis, Michigan State University, East Lansing, 1987.

[45] J. Klingele, S. Dechert, F. Meyer, Coord. Chem. Rev., 2009, 253, 2698-2741.

[46] S. Katsiaouni, S. Dechert, C. Brückner, F. Meyer, Chem. Commun., 2007, 951953.

[47] S. Katsiaouni, S. Dechert, R. P. Briñas, C. Brückner, F. Meyer, Chem. Eur. J., 2008, 14, 4823-4835.

[48] S. Katsiaouni, Ph.D Thesis, Georg-August-Universität, Göttingen, Göttingen, 2007

[49] T. D: Lash, A. M. Young, A. L. von Ruden, G. M. Ferrence, Chem. Commun., 2008, 6309-6311.

[50] A. Sachse, L. Penkova, G. Noël, S. Dechert, O. Varzatskii, I. Fritsky, F. Meyer, Synthesis, 2008, 800-806.

[51] L. Sessler, A. Mozaffari, M. R. Johnson, Org. Synth., 1992, 70, 68-74.

[52] A. Vogel, S. Dechert, M. John, C. Brückner, F. Meyer, Chem. Eur. J., 2016, 22, $2307-2316$.

[53] L. K. Blusch, K. E. Craigo, V. Martin-Diaconescu, A. B. McQuarters, E. Bill, S. Dechert, S. DeBeer, N. Lehnert, F. Meyer, J. Am. Chem. Soc., 2013, 135, 13892-13899. 
[54] L. K. Blusch, Y. Hemberger, K. Pröpper, B. Dittrich, F. Witterauf, M. John, G. Bringmann, C. Brückner, F. Meyer, Chem. Eur. J., 2013, 19, 5868-5880.

[55] L. K. Blusch, O. Mitevski, V. Martin-Diaconescu, K. Pröpper, S. DeBeer, S. Dechert, F. Meyer, Inorg. Chem., 2014, 53, 7876-7885.

[56] K. M. Kadish, M. M. Morrison, Bioinorg. Chem., 1977, 7, 107-115.

[57] D. Chang, T. Malinski, A. Ulman, K. M. Kadish, Inorg. Chem., 1984, 23, 817-824.

[58] K. M. Kadish, K. M. Smith, R. Guilard, Database of redox potentials and binding constants, volume 9 of The porphyrin handbook, Academic Press, San Diego, Calif., 2000.

[59] L. Que, W. B. Tolman, Nature, 2008, 455, 333-340.

[60] M. Costas, M. P. Mehn, M. P. Jensen, J. L. Que, Chem. Rev., 2004, 104, 939-986.

[61] B. J. Wallar, J. D. Lipscomb, Chem. Rev., 1996, 96, 2625-2658.

[62] D. M. Kurtz Jr., J. Biol. Inorg. Chem., 1997, 2, 159-167.

[63] M. Merkx, D. A. Kopp, M. H. Sazinsky, J. L. Blazyk, J. Müller, S. J. Lippard, Angew. Chem. Int. Ed., 2001, 40, 2782-2807.

[64] I. Schlichting, Science, 2000, 287, 1615-1622.

[65] J. Rittle, J. M. Younker, M. T. Green, Inorg. Chem., 2010, 49, 3610-3617.

[66] J. Rittle, M. T. Green, Science, 2010, 330, 933-937.

[67] R. Davydov, N. Strushkevich, D. Smil, A. Yantsevich, A. Gilep, S. Usanov, B. M. Hoffman, Biochemistry, 2015, 54, 7089-7097.

[68] A. Messerschmidt, editor, Handbook of metalloproteins, Wiley, Chichester, 2001.

[69] E. I. Solomon, T. C. Brunold, M. I. Davis, J. N. Kemsley, S.-K. Lee, N. Lehnert, F. Neese, A. J. Skulan, Y.-S. Yang, J. Zhou, Chem. Rev., 2000, 100, 235-350.

[70] R. L. Radin, A. Bino, P. Poganiuch, W. B. Tolman, S. Liu, S. J. Lippard, Angew. Chem. Int. Ed., 1990, 29, 812-814.

[71] M. Torrent, D. G. Musaev, K. Morokuma, J. Phys. Chem. B, 2001, 105, 322-327.

[72] R. E. Stenkamp, Chem. Rev., 1994, 94, 715-726.

[73] James E. Huheey, Ellen A. Keiter, Richard L. Keiter, Anorganische Chemie Prinzipien von Struktur und Reaktivität, De Gruyter, Berlin, 2012.

[74] B. G. Fox, W. A. Froland, J. E. Dege, J. D. Lipscomb, J. Biol. Chem., 1989, 264 , 10023-10033. 
[75] K. E. Liu, A. M. Valenteine, D. Wang, B. H. Huynh, D. E: Edmondson, A. Salifoglou, S. J. Lippard, J. Am. Chem. Soc., 1995, 117, 10174-10185.

[76] B. Burger, S. Demeshko, E. Bill, S. Dechert, F. Meyer, Angew. Chem. Int. Ed., 2012, 51, 10045-10049.

[77] S.-K. Lee, J. D: Lipscomb, Biochemistry, 1999, 39, 4423-4432.

[78] B. J. Brazeau, J. D. Lipscomb, Biochemistry, 2000, 39, 13503-13515.

[79] S.-K. Lee, B. G. Fox, W. A. Froland, J. D. Lipscomb, E. Münck, J. Am. Chem. Soc., 1993, 115, 6450-6451.

[80] S.-K. Lee, J. C. Nesheim, J. D. Lipscomb, J. Biol. Chem., 1993, 268, 21569-21577.

[81] L. Shu, J. C: Nesheim, K. Kauffmann, E: Münck, J. D. Lipscomb, L. Que, Science, 1997, 275, 515-518.

[82] P. Gütlich, E. Bill, A. X. Trautwein, Mössbauer Spectroscopy and Transition Metal Chemistry, Springer Verlag, Heidelberg, 2011.

[83] J. DuBois, T. J. Mizoguchi, S. J. Lippard, Coord. Chem. Rev., 2000, 200, 443-485.

[84] B. D. Dunietz, M. D. Beachy, Y. Cao, D. A. Whittington, S. J. Lippard, R. A. Friesner, J. Am. Chem. Soc., 2000, 122, 2828-2839.

[85] N. D. Priestley, H. G. Floss, W. A. Froland, J. D. Lipscomb, P. G. Williams, H. Morimoto, J. Am. Chem. Soc., 1992, 114, 7561-7562.

[86] L. K. Blusch, The Siamese-Twin Porphyrin and its copper and nickel complexes: A non-innocent twist, Springer Theses, Recognizing Outstanding Ph.D. Research, Springer International Publishing, Cham, 2013.

[87] Rebecca Zilch, Diploma Thesis, Georg-August-University, Göttingen, 2010.

[88] J. C. Röder, F. Meyer, M. Konrad, S. Sandhöfner, E. Kaifer, H. Pritzkow, Eur. J. Org. Chem., 2001, 2001, 4479-4487.

[89] P. G. M. Wuts, T. W. Greene, Greene's protective groups in organic synthesis, 4th edition, Wiley-Interscience, Hoboken, N.J, 2007.

[90] P. A. Stadler, Helv. Chim. Acta, 1978, 61, 1675-1681.

[91] A. Shih-Yuan Lee, Y.-J. Hu, S.-F. Chu, Tetrahedron, 2001, 57, 2121-2126.

[92] C. Ramesh, N. Ravindranath, B. Das, J. Org. Chem., 2003, 68, 7101-7103.

[93] T. Nishiguchi, C. Kamio, J. Chem. Soc., Perkin Trans. 1, 1989, 707.

[94] J. H. Han, Y. E. Kwon, J.-H. Sohn, D. H. Ryu, Tetrahedron, 2010, 66, 1673-1677. 
[95] A. Vogel, Ph.D Thesis, Georg-August-University, Göttingen, 2016.

[96] S. S. Eaton, G. R. Eaton, J. Am. Chem. Soc., 1975, 97, 3660-3666.

[97] S. S. Eaton, G. R. Eaton, J. Am. Chem. Soc., 1977, 99, 6594-6599.

[98] Y. Okuno, T. Kamikado, S. Yokoyama, S. Mashiko, J. Mol. Struct.: THEOCHEM, 2002, 594, 55-60.

[99] P. J. Spellane, M. Gouterman, A. Antipas, S. Kim, Y. C. Liu, Inorg. Chem., 1980, 19, 386-391.

[100] P. G. Gassman, A. Ghosh, J. Almlof, J. Am. Chem. Soc., 1992, 114, 9990-10000.

[101] G. Hariprasad, S. Dahal, B. G. Maiya, J. Chem. Soc., Dalton Trans., 1996, 3429.

[102] J. B. Kim, J. J. Leonard, F. R. Longo, J. Am. Chem. Soc., 1972, 94, 3986-3992.

[103] R. Plamont, Y. Kikkawa, M. Takahashi, M. Kanesato, M. Giorgi, A. Chan Kam Shun, C. Roussel, T. S. Balaban, Chem. Eur. J., 2013, 19, 11293-11300.

[104] J. Yang, P. Huang, Chem. Mater., 2000, 12, 2693-2697.

[105] C. Hansch, A. Leo, R. W. Taft, Chem. Rev., 1991, 91, 165-195.

[106] M. Ishida, S.-J. Kim, C. Preihs, K. Ohkubo, J. M. Lim, B. S. Lee, J. S. Park, V. M. Lynch, V. V. Roznyatovskiy, T. Sarma, P. K. Panda, C.-H. Lee, S. Fukuzumi, D. Kim, J. L. Sessler, Nat. Chem., 2013, 5, 15-20.

[107] J.-Y. Shin, H. Furuta, A. Osuka, Angew. Chem. Int. Ed., 2001, 40, 619-621.

[108] J. Pellegrino, S. E. Bari, D. E. Bikiel, F. Doctorovich, J. Am. Chem. Soc., 2010, 132, 989-995.

[109] A. Srinivasan, T. Ishizuka, A. Osuka, H. Furuta, J. Am. Chem. Soc., 2003, 125, 878-879.

[110] J.-Y. Shin, H. Furuta, K. Yoza, S. Igarashi, A. Osuka, J. Am. Chem. Soc., 2001, 123, 7190-7191.

[111] S. Saito, K. Furukawa, A. Osuka, J. Am. Chem. Soc., 2010, 132, 2128-2129.

[112] B. Hu, J. Li, Angew. Chem. Int. Ed., 2015, 54, 10579-10582.

[113] Y. Han, H. Fang, H. Jing, H. Sun, H. Lei, W. Lai, R. Cao, Angew. Chem. Int. Ed., 2016, $55,5457-5462$.

[114] A. Wolberg, Isr. J. Chem., 1974, 12, 1031-1035.

[115] K. M. Kadish, M. Lin, E. van Caemelbecke, G. de Stefano, C. J. Medforth, D. J. Nurco, N. Y. Nelson, B. Krattinger, C. M. Muzzi, L. Jaquinod, Y. Xu, D. C. Shyr, K. M. Smith, J. A. Shelnutt, Inorg. Chem., 2002, 41, 6673-6687. 
[116] A. Vogel, unpublished results, Georg-August-University, Göttingen, 2016.

[117] D. Sakow, D. Baabe, B. Böker, O. Burghaus, M. Funk, C. Kleeberg, D. Menzel, C. Pietzonka, M. Bröring, Chem. Eur. J., 2014, 20, 2913-2924.

[118] Y. Shen, U. Ryde, 2005, 11, 1549-1564.

[119] K. L. Kostka, B. G. Fox, M. P. Hendrich, T. J. Collins, C. E. F. Rickard, L. J. Wright, E. Münck, J. Am. Chem. Soc., 1993, 115, 6746-6757.

[120] L. A. Yatsunyk, F. A. Walker, Inorg. Chem., 2004, 43, 757-777.

[121] T. R. Dugan, E. Bill, K. C. MacLeod, G. J. Christian, R. E. Cowley, W. W. Brennessel, S. Ye, F. Neese, P. L. Holland, J. Am. Chem. Soc., 2012, 134, 2035220364 .

[122] O. Kahn, Molecular magnetism, Wiley-VCH, New York, 2001.

[123] J. B. Goodenough, Phys. Rev., 1968, 171, 466-479.

[124] M. Vijayakumar, M. S. Gopinathan, J. Mol. Struct.: THEOCHEM, 1996, 361, $15-19$.

[125] J. M. Hollas, Modern spectroscopy, 4th edition, Wiley, Chichester, 2010.

[126] P. W. Atkins, J. de Paula, Physikalische Chemie, 4th edition, Wiley-VCH, Weinheim, 2012.

[127] Y. Ling, Y. Zhang, J. Am. Chem. Soc., 2009, 131, 6386-6388.

[128] J. S. Griffith, Discuss. Faraday Soc., 1958, 26, 81.

[129] D. H. Dolphin, J. R. Sams, T. B. Tsin, Inorg. Chem., 1977, 16, 711-713.

[130] H. Ogoshi, H. Sugimoto, E.-i. Watanabe, Z.-i. Yoshida, Y. Maeda, H. Sakai, Bull. Chem. Soc. Jpn., 1981, 54, 3414-3419.

[131] T. Kaczmarzyk, K. Dziedzic-Kocurek, I. Rutkowska, K. Dziliński, NUKLEONIKA, 2015, 60, 57-61.

[132] C. A. Reed, T. Mashiko, S. P. Bentley, M. E. Kastner, W. R. Scheidt, K. Spartalian, G. Lang, J. Am. Chem. Soc., 1979, 101, 2948-2958.

[133] V. Schünemann, M. Gerdan, A. X. Trautwein, N. Haoudi, D. Mandon, J. FISCHER, R. WEISS, A. Tabard, R. Guilard, Angew. Chem. Int. Ed., 1999, 38, $3181-3183$

[134] T. Ikeue, Y. Ohgo, T. Yamaguchi, M. Takahashi, M. Takeda, M. Nakamura, Angew. Chem. Int. Ed., 2001, 40, 2617-2620. 
[135] M. M. Maltempo, J. Chem. Phys., 1974, 61, 2540.

[136] R. L. Cohen, K. W. West, Chem. Phys. Lett., 1972, 13, 482-484.

[137] N. N. Greenwood, T. C. Gibb, Mössbauer Spectroscopy, Springer Netherlands, Dordrecht, 1971.

[138] K. M. Kadish, M. M. Morrison, L. A. Constant, L. Dickens, D. G. Davis, J. Am. Chem. Soc., 1976, 98, 8387-8390.

[139] A. M. Stolzenberg, S. H. Strauss, R. H. Holm, J. Am. Chem. Soc., 1981, 103, 4763-4778.

[140] K. M. Kadish, E. van Caemelbecke, F. D’Souza, C. J. Medforth, K. M. Smith, A. Tabard, R. Guilard, Inorg. Chem., 1995, 34, 2984-2989.

[141] N. G. Connelly, W. E. Geiger, Chem. Rev., 1996, 96, 877-910.

[142] L. A. Bottomley, K. M. Kadish, Inorg. Chem., 1981, 20, 1348-1357.

[143] K. M. Kadish, The Electrochemistry of Metalloporphyrins in Nonaqueous Media, in S. J. Lippard, editor, Progress in Inorganic Chemistry, volume 34 of Progress in Inorganic Chemistry, John Wiley \& Sons, Inc, Hoboken, NJ, USA, 1986, 435-605.

[144] D. Lexa, M. Momenteau, J. Mispelter, J. M. Lhoste, Bioelectrochem. Bioenerg., 1974, 1, 108-117.

[145] L. A. Constant, D. G. Davis, Anal. Chem., 1975, 47, 2253-2260.

[146] R. Crescenzi, E. Solari, C. Floriani, A. Chiesi-Villa, C. Rizzoli, J. Am. Chem. Soc., 1999, 121, 1695-1706.

[147] C. Hu, J. An, B. C. Noll, C. E. Schulz, W. R. Scheidt, Inorg. Chem., 2006, 45, $4177-4185$

[148] E. McCandlish, A. R. Miksztal, M. Nappa, A. Q. Sprenger, J. S. Valentine, J. D. Stong, T. G. Spiro, J. Am. Chem. Soc., 1980, 102, 4268-4271.

[149] K. Dürr, O. Troeppner, J. Olah, J. Li, A. Zahl, T. Drewello, N. Jux, J. N. Harvey, I. Ivanović-Burmazović, Dalton Trans., 2012, 41, 546-557.

[150] C. A. Reed, T. Mashiko, W. R. Scheidt, K. Spartalian, G. Lang, J. Am. Chem. Soc., 1980, 102, 2302-2306.

[151] D. Dolphin, J. R. Sams, T. B. Tsin, K. L. Wong, J. Am. Chem. Soc., 1976, 98, 6970-6975.

[152] S. H. Strauss, R. H. Holm, Inorg. Chem., 1982, 21, 863-868. 
[153] C. Hu, A. Roth, M. K. Ellison, J. An, C. M. Ellis, C. E. Schulz, W. R. Scheidt, J. Am. Chem. Soc., 2005, 127, 5675-5688.

[154] J. P. Collman, J. L. Hoard, N. Kim, G. Lang, C. A. Reed, J. Am. Chem. Soc., 1975, 97, 2676-2681.

[155] H. Kobayashi, Y. Maeda, Y. Yanagawa, BCSJ, 1970, 43, 2342-2346.

[156] K. Ôno, A. Ito, T. Fujita, J. Phys. Soc. Jpn., 1964, 19, 2119-2126.

[157] C. E. Johnson, Proc. Phys. Soc., 1966, 88, 943-955.

[158] B. B. Wayland, L. F. Mehne, J. Swartz, J. Am. Chem. Soc., 1978, 100, 2379-2383.

[159] T. Hashimoto, J. E. Baldwin, F. Basolo, R. L. Dyer, M. J. Crossley, J. Am. Chem. Soc., 1982, 104, 2101-2109.

[160] C. Swistak, K. M. Kadish, Inorg. Chem., 1987, 26, 405-412.

[161] K. Seufert, M.-L. Bocquet, W. Auwarter, A. Weber-Bargioni, J. Reichert, N. Lorente, J. V. Barth, Nat. Chem., 2011, 3, 114-119.

[162] K. E. Dalle, T. Gruene, S. Dechert, S. Demeshko, F. Meyer, J. Am. Chem. Soc., 2014, 136, 7428-7434.

[163] N. Kindermann, E. Bill, S. Dechert, S. Demeshko, E. J. Reijerse, F. Meyer, Angew. Chem. Int. Ed., 2015, 54, 1738-1743.

[164] N. Kindermann, S. Dechert, S. Demeshko, F. Meyer, J. Am. Chem. Soc., 2015, $137,8002-8005$.

[165] A. Bencini, D. Gatteschi, Electron paramagnetic resonance of exchange coupled systems, Springer, Berlin, 1990.

[166] D. Dolphin, D. J. Halko, E. Johnson, Inorg. Chem., 1981, 20, 4348-4351.

[167] T. Shiba, D. Kuroda, T. Kurahashi, S. Matsubara, Synlett, 2014, 25, 2005-2008.

[168] Autorenkollektiv, The Porphyrin Handbook, volume 3, Acedemic Press, San Diego, 2000.

[169] F. A. Walker, J. Am. Chem. Soc., 1970, 92, 4235-4244.

[170] F. A. Walker, J. Magn. Resonan., 1974, 15, 201-218.

[171] K. M. Kadish, K. M. Smith, R. Guilard, NMR and EPR, volume 5 of The porphyrin handbook, Academic Press, San Diego, Calif., 2000.

[172] D. Kim, J. Cho, Y.-M. Lee, R. Sarangi, W. Nam, Chem. Eur. J., 2013, 19, 14112 14118 . 
[173] A. M. Clover, J. Am. Chem. Soc., 1923, 45, 3133-3138.

[174] Ullmann's Encyclopedia of Industrial Chemistry, Wiley-VCH Verlag GmbH \& Co. KGaA, Weinheim, Germany, 2000.

[175] N. V. Chizhova, R. S. Kumeev, N. Z. Mamardashvili, Russ. J. Inorg. Chem., 2013, $58,740-743$

[176] K. M. Kadish, X. H. Mu, X. Q. Lin, Inorg. Chem., 1988, 27, 1489-1492.

[177] I. Klawitter, M. R. Anneser, S. Dechert, S. Meyer, S. Demeshko, S. Haslinger, A. Pöthig, F. E. Kühn, F. Meyer, Organometallics, 2015, 34, 2819-2825.

[178] S. Meyer, I. Klawitter, S. Demeshko, E. Bill, F. Meyer, Angew. Chem. Int. Ed., 2013, 52, 901-905.

[179] C. Kupper, A. Schober, S. Demeshko, M. Bergner, F. Meyer, Inorg. Chem., 2015, 54, 3096-3098.

[180] M. Selke, M. F. Sisemore, J. S. Valentine, J. Am. Chem. Soc., 1996, 118, 20082012.

[181] K. Dürr, B. P. Macpherson, R. Warratz, F. Hampel, F. Tuczek, M. Helmreich, N. Jux, I. Ivanović-Burmazović, J. Am. Chem. Soc., 2007, 129, 4217-4228.

[182] K. Dürr, J. Olah, R. Davydov, M. Kleimann, J. Li, N. Lang, R. Puchta, E. Hübner, T. Drewello, J. N. Harvey, N. Jux, I. Ivanović-Burmazović, Dalton Trans., 2010, 39, 2049.

[183] K. Dürr, N. Jux, A. Zahl, R. van Eldik, I. Ivanović-Burmazović, Inorg. Chem., 2010, 49, 11254-11260.

[184] A. Franke, C. Fertinger, R. van Eldik, Chem. Eur. J., 2012, 18, 6935-6949.

[185] S. P. de Visser, J. S. Valentine, W. Nam, Angew. Chem. Int. Ed., 2010, 49, 20992101.

[186] M. T. Kieber-Emmons, M. F. Qayyum, Y. Li, Z. Halime, K. O. Hodgson, B. Hedman, K. D. Karlin, E. I. Solomon, Angew. Chem. Int. Ed., 2012, 51, 168-172.

[187] G. M. Sheldrick, Acta Cryst. C Struct. Chem., 2015, 71, 3-8.

[188] G. M. Sheldrick, Acta Cryst. C Struct. Chem., 2015, 71, 3-8.

[189] W. L. F. Armarego, D. D. Perrin, Purification of laboratory chemicals, 4th edition, Butterworth-Heinemann, Oxford, 2002.

[190] V. J. Arán, M. Kumar, J. Molina, L. Lamarque, P. Navarro, E. García-España, J. A. Ramírez, S. V. Luis, B. Escuder, J. Org. Chem., 1999, 64, 6135-6146.

[191] A. L. Spek, Acta Cryst. C Struct. Chem., 2015, 71, 9-18. 


\section{Acknowledgement}

At the beginning I would like to thank Prof. Dr. Franc Meyer for his support, the challenging and versatile project, the scientific freedom and his guidance and advice. Furthermore I would like to thank him for the opportunity and the financial support to do research at the University of Connecticut under the guidance of Prof. Dr. Christian Brückner. With respect to this, I would like to thank Prof. Dr. Christian Bückner for his support, the outstanding collaboration, his expertise in porphyrin chemistry, the possibilty to accommodate me in his laboratory and the financial support. Moreover, I would like to thank Prof. Dr. Sven Schneider for being my correferent.

Additionally I would like to thank Dr. Eckhard Bill for the fruitful and helpful discussions and measurements of EPR and Mößbauer samples. Further on, I would like to thank Dr. Serhiy Demeshko for his expertise in SQUID measurements, Mößbauer spectroscopy and the huge number of measurements as well as Dr. Sebastian Dechert for this support in DFT calculations, the measurements of my solid state structures and the variety of crystallization attemps he inspected. I would also like to thank Dr. Claudia Stückl for the measurements of EPR spectra, the scientific discussions and the guidance during my teaching assistance, especially in the practical courses. I thank Jörg Teichgräber for cyclic voltammetry measurements and all the interesting conversations during our labtime together. Additionally, I would like to thank Ralf Schöne for all NMR measurements and the analytic department for mass spectrometry, especially Dr. Holm Frauendorf, Thomas Schuchardt and Frank Hambloch.

I would like to thank my labmates, especially Anastasia, Jann, Alex, Jörg, Dr. Kai and Dr. Morkas for the nice time during the last years. Additionally, I would like to thank the "Coffee Club" for all the interesting discussions, all the fun we had and especially for the caffeine support. Further on, I thank my labmates from the laboratory of Prof. Dr. Christian Brückner in Storrs and all the new friends I made in the U.S. for the nice four months and the ongoing friendships over the last years. It was an amazing time with all of you. I thank all my friends in Göttingen, especially Falko, Patrick, Jonathan and Basti and my friends from my schooldays, Malte, Tim, Olli Jones and Thomas for their unlimited support. I further would like to thank Basti for his outstanding personality and the patience he always had with me during hard times in the last years.

Additionally, I would like to thank all the proofreaders of this thesis Anne, Anastasia, Ali, Patrick, Alex and Falko. 
Finally I would like to thank my familiy for their strong continuous support during the last years. Without their support I would not be where I am now. 